Fabrication and Doping of Silicon Micropillar Arrays for Solar Light Harvesting

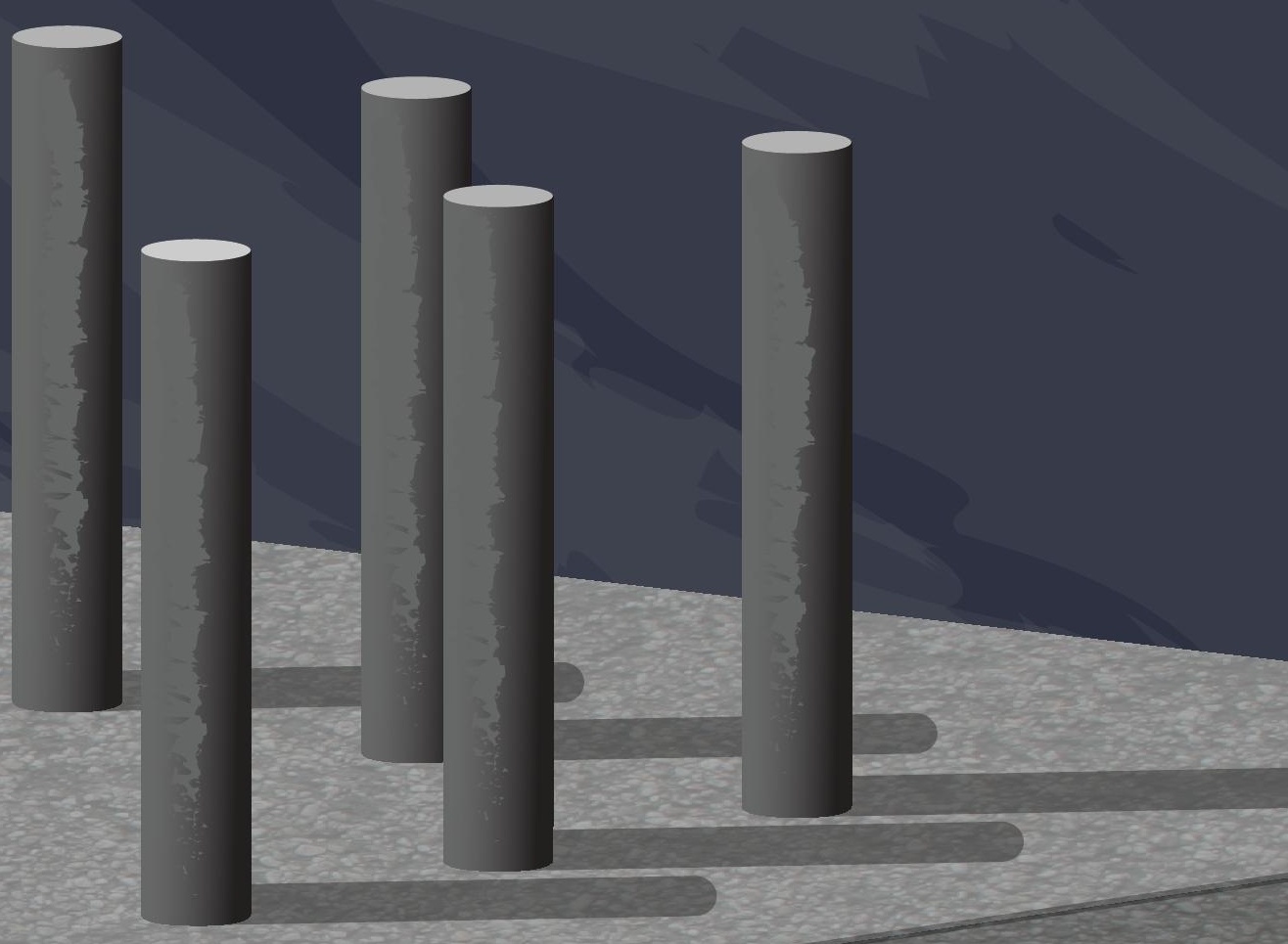

Rick Elbersen 


\section{FABRICATION AND DOPING OF SILICON MICROPILLAR ARRAYS FOR SOLAR LIGHT HARVESTING}

Rick Elbersen 


\section{Promotiecommissie:}

Prof. dr. ir. Hans Hilgenkamp (voorzitter) Universiteit Twente

Prof. dr. ir. Jurriaan Huskens (promotor) Universiteit Twente

Prof. dr. Han Gardeniers (promotor) Universiteit Twente

Prof. dr. Marlies van Bael Universiteit Hasselt

Prof. dr. Ernst Sudhölter Technische Universiteit Delft

Prof. dr. ir. Wilfred van der Wiel Universiteit Twente

Prof. dr. Guido Mul Universiteit Twente

Dr. ir. Mark Huijben Universiteit Twente

This work is part of the research programme of the Foundation for Fundamental Research on Matter (FOM, project 115-10TBSC07-2), which is part of the Netherlands Organization for Scientific Research (NWO). It was carried out within the framework of the national program on BioSolar Cells, co-financed by the Dutch Ministry of Economic Affairs, Agriculture, and Innovation.

Fabrication and doping of silicon micropillar arrays for solar light harvesting

ISBN: 978-90-365-4007-0

DOI: $10.3990 / 1.9789036540070$

Cover art: Martin Binnema

Printed by: Gildeprint - The Netherlands

(C) Copyright 2015 Rick Elbersen 


\title{
FABRICATION AND DOPING OF SILICON MICROPILLAR ARRAYS FOR SOLAR LIGHT HARVESTING
}

\author{
PROEFSCHRIFT
}

\author{
ter verkrijging van \\ de graad van doctor aan de Universiteit Twente, \\ op gezag van de rector magnificus, \\ prof. dr. H. Brinksma, \\ volgens besluit van het College voor Promoties \\ in het openbaar te verdedigen \\ op vrijdag 4 december 2015 om 16.45 uur
}

door

Rick Elbersen

geboren op 6 januari 1987

te Deurne, Nederland 
Dit proefschrift is goedgekeurd door de promotoren:

Prof. dr. ir. Jurriaan Huskens (promotor)

Prof. dr. Han Gardeniers (promotor) 


\section{Table of Contents}

Chapter 1 Solar Energy Applications of Silicon......................................... 9

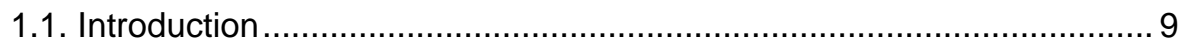

1.2. Aims of the research.................................................................. 13

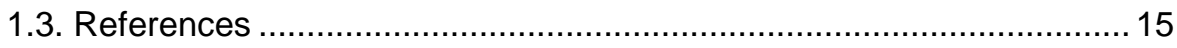

Chapter 2 Fabrication and Doping Methods for Silicon Nano- and Micropillar Arrays for Solar Light Harvesting: A Review .........................17

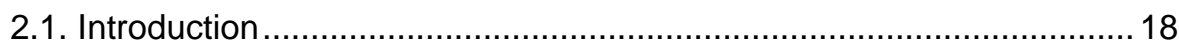

2.2. Optimized micro/nanopillar designs for solar-to-fuel conversion ......... 18

2.3. Fabrication of silicon nano/micropillars ............................................. 22

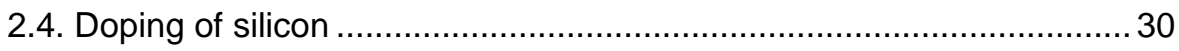

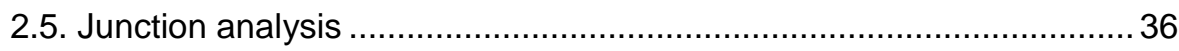

2.6. Optical and electrical characterization .............................................. 41

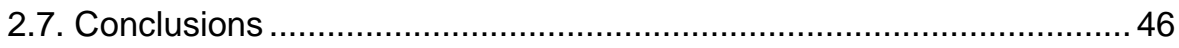

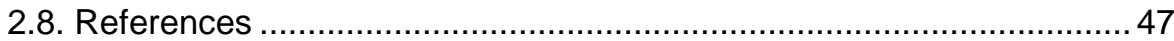

Chapter 3 Controlled Doping Methods for Radial $\mathbf{p} / \mathbf{n}$ Junctions in Silicon

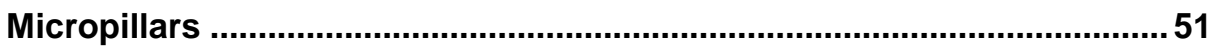

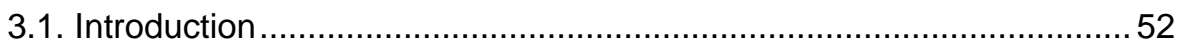

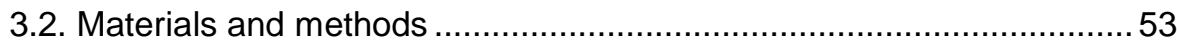

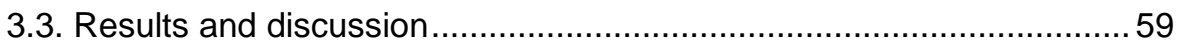

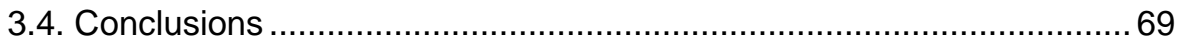

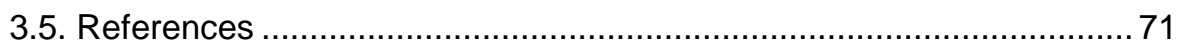

Chapter 4 Effects of Pillar Height and Junction Depth on the Performance of Radially Doped Silicon Pillar Arrays for Solar Energy Applications ...73

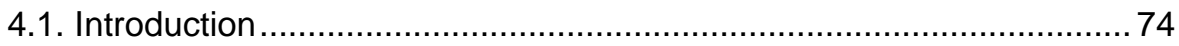

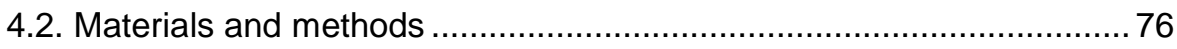




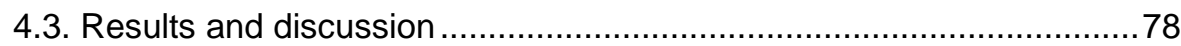

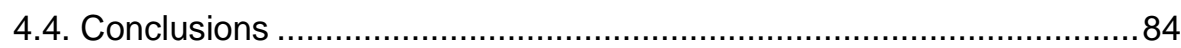

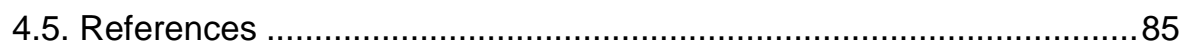

Chapter 5 Electrical Characterization of Silicon Micropillars with Radial p/n Junctions Containing Passivation and Anti-Reflection Coatings......87

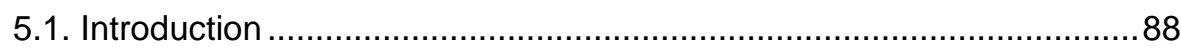

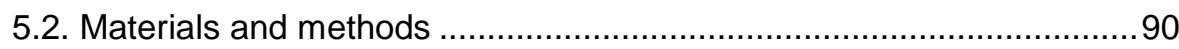

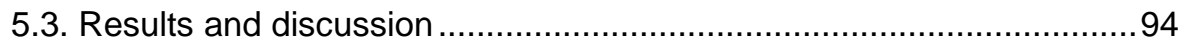

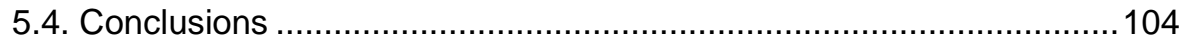

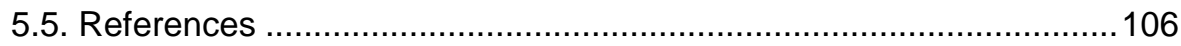

Chapter 6 Spatioselective Electrochemical and Photoelectrochemical Functionalization of Silicon Microwires with Axial p/n Junctions .........107

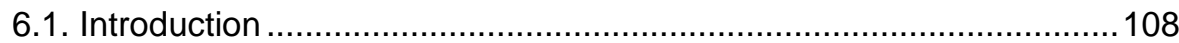

6.2. Materials and methods ….........................................................110

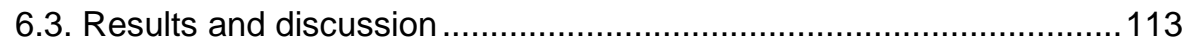

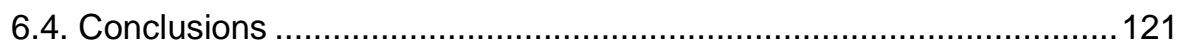

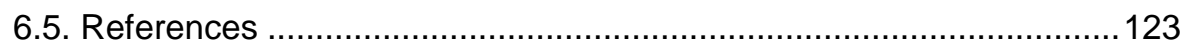

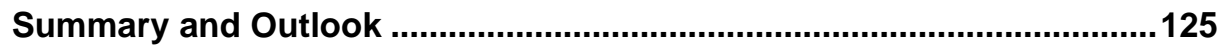

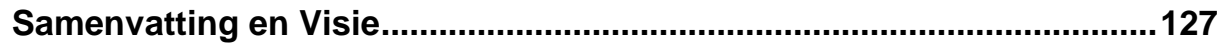

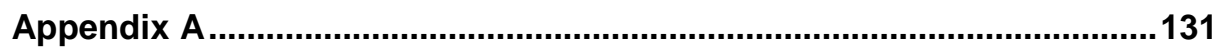

A.1. Process flow radial junctions ...................................................... 131

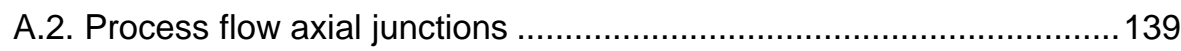

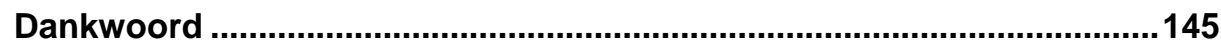

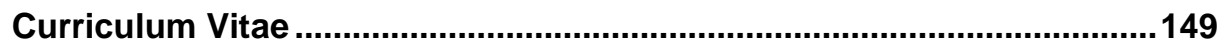

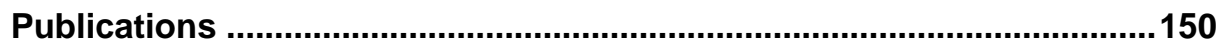






\section{Chapter 1 \\ Solar Energy Applications of Silicon}

\subsection{Introduction}

One of the world's major challenges currently is to switch from an oil-based economy to a more sustainable alternative energy economy. Many different types of renewable energy, such as wind energy, biomass, blue energy and solar energy, are gaining interest and start to compete with fossil fuels. For example, the electrical power generated by the use of solar energy in the Netherlands increased twelve-fold between 2005 and 2013. ${ }^{[1]}$ In addition, under the 2009 EU Renewable Energy Directive, The Netherlands has committed to provide at least $14 \%$ of their total energy consumption from renewable energy in 2020. ${ }^{[2]}$ To achieve this goal, a great effort in both research and business is required to make renewable energy sources more attractive, and this is a trend that is visible worldwide.

One of the possible sustainable alternatives is the photovoltaic (PV) cell, which has already been under investigation since the first $\mathrm{p} / \mathrm{n}$ junction was fabricated in 1954 in the Bell laboratories. ${ }^{[3]}$ In a PV cell, light is converted into electricity in three stages. First, the light is absorbed, generating an electron/hole pair. Secondly, the electron/hole pair is separated, and finally the charge carriers are extracted from the PV cell by an external circuit. PV cells can use the light of the sun as an energy source, meaning that there is basically an unlimited source of energy available. PV cells can be made from a variety of materials, for example crystalline silicon, gallium arsenide and organic materials. All of these different materials have already been subjected to intensive research, as shown by the National Renewable Energy Laboratory (NREL, based in the US). Since 1975, they have been keeping track of best research-cell efficiencies, as shown in Figure 1.1. ${ }^{[4]}$ 


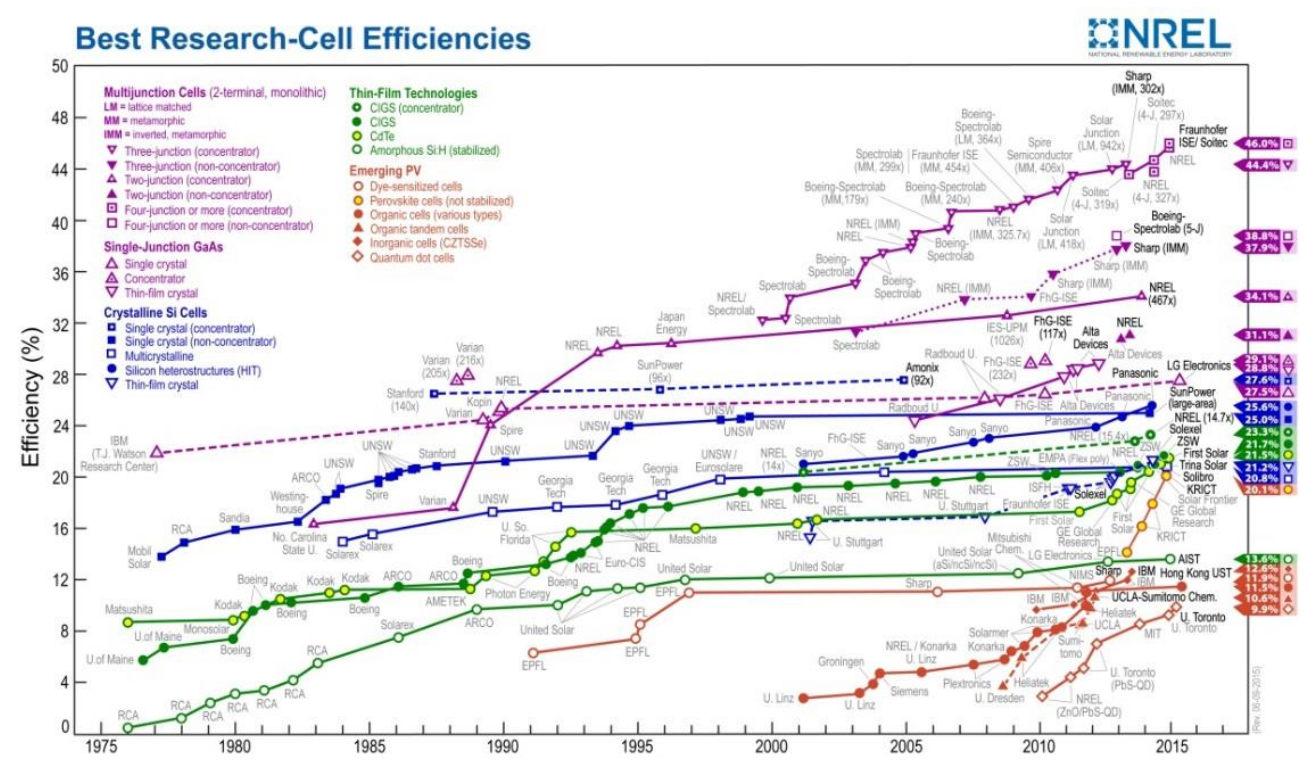

Figure 1.1: Overview of the best performing solar cells (in terms of efficiency) for various materials and setups. ${ }^{[4]}$

The efficiencies shown in Figure 1.1 are based on research-scale devices, and the figure provides no information about the ease of fabrication or the total cost per area unit or per kWh, meaning that the highest efficiency is not always the most practical option. From a commercial point of view, silicon is by far the most used material for PV cells, as it accounts for about $90 \%$ of the total production, as of 2013. ${ }^{[5]}$ Silicon has several advantageous properties, for example, it is abundant, non-toxic, low cost and widely known in the nano/micro-fabrication world. The availability of different fabrication and deposition techniques to modify silicon surfaces offers many possibilities to enhance the PV characteristics of silicon. ${ }^{[6-8]}$

Unfortunately, the production of solar electricity rarely matches to the actual demand. On a sunny afternoon, there is a surplus of energy, whereas during the night there is a shortage, as there is no electricity generated. This imbalance between production and demand is schematically shown in Figure 1.2. To prevent loss of a large excess of solar power, the surplus electricity can be stored in batteries or used to generate a fuel, for example, by coupling a solar cell to an electrolyzer. 


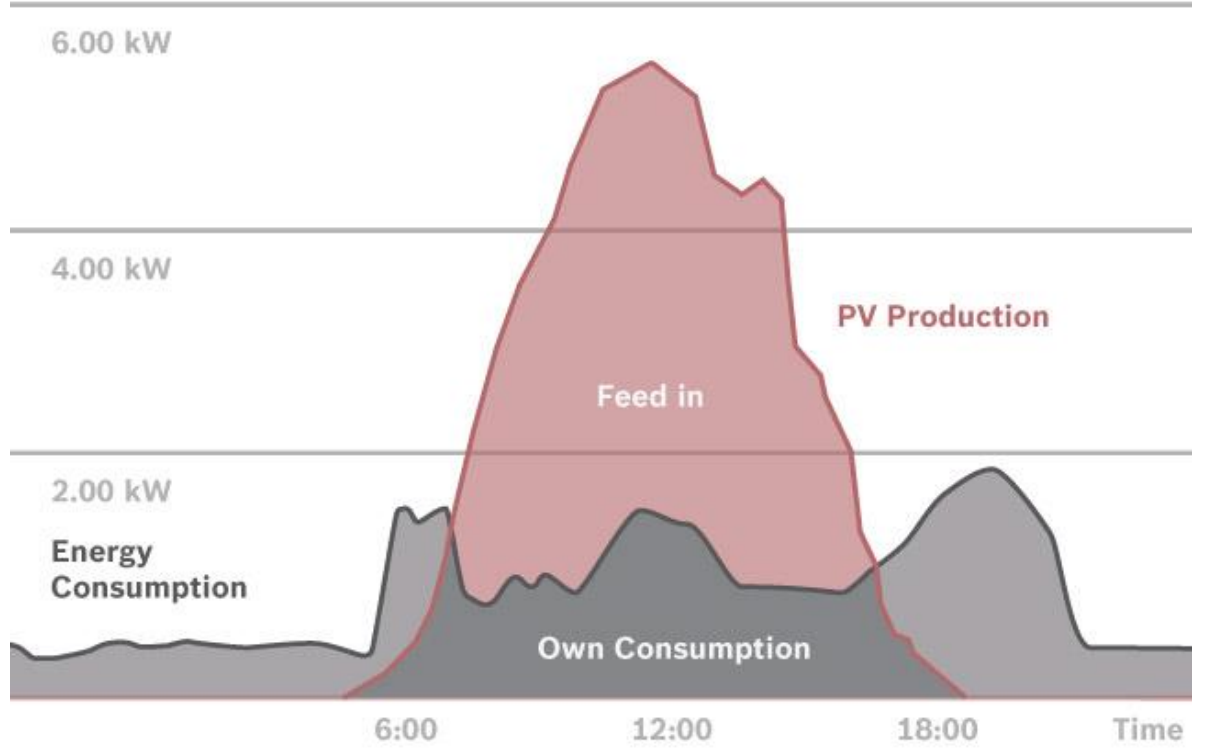

Figure 1.2: Expected solar energy production and demand over a day, on an average sunny day. ${ }^{[9]}$

Another solution would be to make an integrated device that captures light to split water into oxygen and hydrogen as the fuel. The latter is known as a solar-to-fuel (S2F) device, and it already has been proven to function in labscale. ${ }^{[10,11]} \mathrm{S} 2 \mathrm{~F}$ devices could provide the solution to the gap between energy generation and demand, as the chemical energy of a fuel is the most condensed form of energy and can be transported and stored for later use. A general S2F device requires the coupling of various processes, i.e. light harvesting, charge separation and charge carrier transport to different catalysts, for the oxidation of water and reduction of protons. In theory, the splitting of water would require a photovoltage of more than $1.23 \mathrm{~V}$, but practically at least $0.6 \mathrm{~V}$ extra is required, to drive the water oxidation and reduction at normal current densities obtained from 1 sun $\left(15-25 \mathrm{~mA} / \mathrm{cm}^{2}\right) .{ }^{[12]}$ There are two possible options for a S2F device, a single semiconductor absorber or a tandem device, where two semiconductors are connected. The energy scheme for both options is shown in Figure 1.3. In the case of a single semiconductor, a fairly large (>2.0 V) bandgap material is required, and this leaves out much of the solar spectrum. This results in a lower overall 
efficiency, estimated at about $10 \%$ in literature. ${ }^{[12,13]}$ For a tandem device, where two semiconductors are used, two (smaller) bandgaps result in a larger photon collection, at the cost of recombination, as the quantum yield is reduced. For a tandem device with one smaller $(\sim 1.2 \mathrm{eV})$ and larger $(\sim 1.9 \mathrm{eV})$, the maximum practical water splitting efficiency can reach up to $15 \% .{ }^{[12,13]}$ Silicon has a bandgap of $1.1 \mathrm{eV}$, which makes it an interesting candidate for the lower bandgap material.

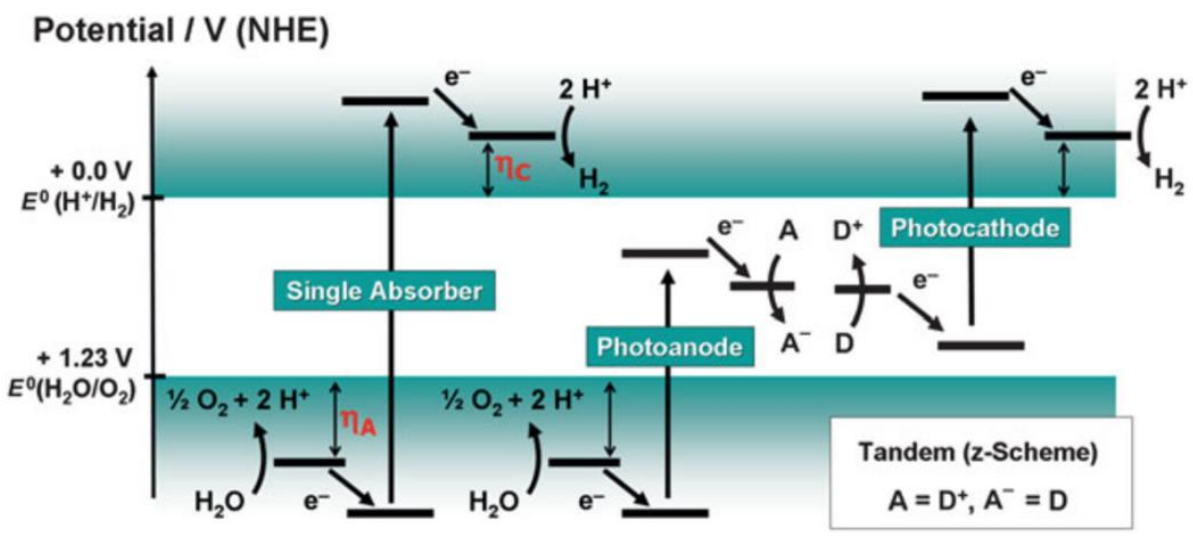

\section{$\eta_{\mathbf{A} / \mathrm{C}}$ Electrochemical Overpotentials}

Figure 1.3: Energy scheme for both a single absorber (left) and a tandem system (right) for photocatalytic water splitting. In addition to the required bandgap width, the bandgap position should also match the required potentials for the water oxidation and the hydrogen reduction steps. A single absorber would require a band gap of $2.0 \mathrm{eV}$, whereas the tandem system can use two lower bandgap materials instead, but the process requires 2 photons for the generation of 1 electron/hole pair. Reproduced with permission. ${ }^{[11]}$ Copyright 2011, Cambridge University Press. 


\subsection{Aims of the research}

In this thesis, we aim to gain knowledge on and improve the fabrication of silicon solar energy devices, in terms of the micro/nano-structuring and doping of silicon as a semiconductor material. In addition, we explore options to further improve the properties and functionalities of PV cells, keeping in mind that these can possibly be used as a platform for S2F devices as well.

The thesis has been structured as follows:

Chapter 2 gives a state-of-the-art literature overview of the fabrication and doping of structured silicon PV cells. Emphasis is placed on the fabrication limitations of common techniques and the analysis of different doping techniques for 3D silicon structures.

In Chapter 3, the fabrication of radially doped $\mathrm{p} / \mathrm{n}$ junctions in silicon micropillars is described. The doping was performed by several methods, such as low-pressure chemical vapor deposition (LPCVD), solid source dotation (SSD) and plasma-enhanced chemical vapor deposition (PECVD). In addition, the formation of the silicon $\mathrm{p} / \mathrm{n}$ junctions was simulated by finite element modeling, and experimentally analyzed on both flat and 3D structures. Finally, the electrical properties of flat and pillar array substrates were compared, for both $\mathrm{p} / \mathrm{n}$ and $\mathrm{n} / \mathrm{p}$ junctions.

The optimization of these radially doped silicon micropillars, in terms of pillar height and junction depth, is reported in Chapter 4. First, the height of the pillars was varied between 0 and $60 \mu \mathrm{m}$, using fabrication and doping methods as described in Chapter 3. Secondly, by adjusting the doping time and temperature, the junction depth was varied between shallow $(140 \mathrm{~nm})$ and large $(1640 \mathrm{~nm})$ depths. The effects of both pillar height and junction depth were analyzed subsequently by electrical measurements.

Chapter 5 continues with the optimization of the silicon micropillar arrays, by the use of a passivation and anti-reflection coating. The reflectivities of thin films of various materials $\left(\mathrm{Al}_{2} \mathrm{O}_{3}, \mathrm{SiO}_{2}\right.$ and $\left.\mathrm{SiN}_{\mathrm{x}}\right)$ were simulated to predict the 
optimal thickness for light trapping. Subsequently these layers were grown on flat silicon wafers, using atomic layer deposition and low-pressure chemical vapor deposition, to verify the simulations. Similar deposition experiments were performed on silicon micropillars, followed by a detailed study by high-resolution scanning electron microscopy to investigate the $3 \mathrm{D}$ deposition characteristics. Finally, the electrical properties were measured and compared to bare samples, to identify and quantify the improvement of the performance achieved by the passivation layers.

In Chapter 6, the possibility to functionalize silicon with metal nanoparticles has been explored, by using the electrodeposition of platinum and silver. Unlike the previous chapters, where silicon micropillars with radial junctions were used, this chapter made use of axial junctions. The selective functionalization of both the $p$ - and n-type parts was proven, where first platinum was deposited on the bottom p-type part, followed by the silver deposition on the top n-type part, without the use of any masking step.

Finally, the main conclusions of the work presented in this thesis are briefly described, followed by an outlook for future research. 


\subsection{References}

[1] Renewable energy; capacity, domestic production and use, 1990-2013 (http://statline.cbs.nl/StatWeb/publication/?DM=SLEN\&PA=71457ENG), Accessed 16th of June, 2015.

[2] Promotion of the use of energy from renewable sources (http://eur-lex.europa.eu/legalcontent/EN/TXT/?qid=1434448431833\&uri=URISERV:en0009), Accessed 16th of June, 2015.

[3] D. M. Chapin, C. S. Fuller, G. L. Pearson, J. Appl. Phys., 1954, 25, 676-677.

[4] Best Research-Cell Efficiencies (http://www.nrel.gov/ncpv/images/efficiency_chart.jpg), Accessed 16th of June, 2015.

[5] Photovoltaics Report Fraunhofer Institute (http://www.ise.fraunhofer.de/de/downloads/pdf-files/aktuelles/photovoltaics-report-inenglischer-sprache.pdf), Accessed 16th of June, 2015.

[6] B. Tian, X. Zheng, T. J. Kempa, Y. Fang, N. Yu, G. Yu, J. Huang, C. M. Lieber, Nature, 2007, 449, 885-889.

[7] S. Pillai, K. R. Catchpole, T. Trupke, M. A. Green, J. Appl. Phys., 2007, 101, 093105

[8] E. Garnett, P. Yang, Nano Lett., 2010, 10, 1082-1087.

[9] Solar power: Generation and own power consumption (http://bosch-solarstorage.com/independence/self-reliance/), Accessed 17th of June, 2015.

[10] S. Y. Reece, J. A. Hamel, K. Sung, T. D. Jarvi, A. J. Esswein, J. J. H. Pijpers, D. G. Nocera, Science, 2011, 334, 645-648.

[11] G. Mul, C. Schacht, W. P. M. van Swaaij, J. A. Moulijn, Chemical Engineering and Processing: Process Intensification, 2012, 51, 137-149.

[12] F. E. Osterloh, B. A. Parkinson, MRS Bulletin, 2011, 36, 17-22.

[13] M. F. Weber, M. J. Dignam, Int. J. Hydrogen Energy, 1986, 11, 225-232. 


\section{Chapter 2 \\ Fabrication and Doping Methods for Silicon Nano- and Micropillar Arrays for Solar Light Harvesting: A Review}

Silicon is one of the main components of commercial solar cells and is used in many other solar light harvesting devices. The overall efficiency of these devices can be increased by the use of structured surfaces that contain nanometer to micrometer sized pillars with radial p/n junctions. High densities of such structures greatly enhance the light absorbing properties of the device, whereas the 3D p/n junction geometry shortens the diffusion length of minority carriers and diminishes recombination. Due to the vast silicon nano- and microfabrication toolbox that exists nowadays, many versatile methods for the preparation of such highly structured samples are available. Furthermore, the formation of $p / n$ junctions on structured surfaces is possible by a variety of doping techniques, in large part transferred from microelectronic circuit technology. The right choice of doping method, to achieve good control of junction depth and doping level, can attribute to an improvement of the overall efficiency that can be obtained in devices for energy applications. This paper presents a review of the state-of-the-art of the fabrication and doping of silicon micro and nanopillars, as well as of the analysis of the properties and geometry of thus formed 3D structured $p / n$ junctions.

This chapter has been adapted from: R. Elbersen, W.J.C. Vijselaar, R.M. Tiggelaar, J.G.E. Gardeniers, J. Huskens, Adv. Mater., 2015, doi: 10.1002/adma.201502632 


\subsection{Introduction}

Since the 1970s, silicon has been under investigation for the use in solar cell applications. Lately, this research has been expanded to the area of solar fuels, where a higher efficiency of the light absorber has a large impact on the total efficiency of the solar-to-fuel device. In this review, we summarize the state-of-the-art of fabrication and doping methods for nano- and micro-sized silicon pillar arrays. A discussion is provided of the optimal design parameters for such devices, based on computational modeling work. This is followed by an overview of techniques used to fabricate arrays of silicon nano- and/or micropillars. Finally, an evaluation is given of the different doping methods that exist for the creation of $\mathrm{p} / \mathrm{n}$ junctions in silicon micro or nanostructures and of the techniques available to analyze such junctions, with a focus on doping of 3D structures.

\subsection{Optimized micro/nanopillar designs for solar-to-fuel conversion}

Recently, pillar arrays of silicon have gained attention for solar energy applications because of their increased light harvesting properties, compared to flat surfaces. This is due to their higher surface area and efficient light trapping by multiple interactions of the light within the gaps between the pillars, even at relatively small pillar aspect ratios. Furthermore, if the pillars have a radial junction, the effective junction area is increased, which leads to enhanced charge carrier generation and separation. Finally and perhaps most importantly, the radial junction decouples the charge transport and light incidence direction, reducing the chance of recombination. ${ }^{[1-3]}$ In order to obtain the best possible solar cell/solar-to-fuel devices, many parameters need to be optimized, such as the emitter (introduced doping layer) and base wafer doping levels, junction depth and profile, as well as the dimensions of the pillars, in terms of height, pitch and diameter. A typical design consists of 
arrays of nano- or micro-sized pillars, with contacts to measure or extract the current and to control the voltage.

\subsection{1. $\mathrm{P} / \mathrm{n}$ junctions in silicon}

The use of a radial junction in silicon pillars is the most efficient junction orientation, as was elaborated theoretically by Kayes et al. in $2005,{ }^{[4]}$ and is therefore the main geometry considered in this review. Simulations of the effect of junction depth on the efficiency of solar cells were performed in 1991, in the work of Durán, where a flat silicon solar cell was simulated in order to predict the optimum junction depth at a given surface dopant concentration. ${ }^{[5]}$ Although the difference between the best and least performing junction depths ( $0.4 \mu \mathrm{m}$ vs $1.0 \mu \mathrm{m}$, respectively) is small ( $18.6 \%$ vs $18.3 \%)$, their data shows a trend that thinner junctions perform slightly better. In more recent publications on simulations of junctions depths, this trend is confirmed: smaller junction depths result in higher currents, and thus higher overall efficiencies. ${ }^{[6-8]}$ Figure 2.1 shows an overview of the influence of the emitter doping level, the junction depth and minority carrier diffusion length, on the open circuit voltage $\left(V_{O C}\right)$ and short-circuit current density $\left(J_{S C}\right)$ for a textured solar cell. ${ }^{[6]}$ The authors suggested an optimized emitter doping of $1 \times 10^{20} \mathrm{~cm}^{-3}$, as a trade-off between a higher $V_{O C}$ and efficiency on the one hand and the manufacturing feasibility of such high doping levels on the other hand. In addition to a high doping concentration, a shallow junction depth should be used, however, no numerical limitation was proposed. ${ }^{[6]}$ Overall, simulations indicate that a thin junction, combined with a high surface doping level, is most efficient. ${ }^{[6]} \mathrm{A}$ high dopant concentration in the junction ensures better charge separation and therewith a higher open circuit voltage. However, a high doping level also reduces the minority carrier diffusion path length and enhances the amount of recombination sites, and the thicker the junction the stronger this effect will be. 

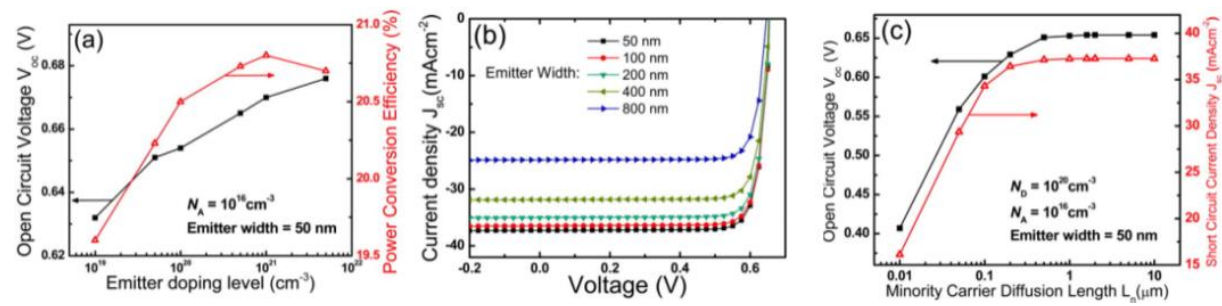

Figure 2.1: Simulations of various parameters of a textured silicon solar cell. (a) $J_{S C}$ and overall efficiency as a function of the emitter doping level, (b) $J V$ characteristics for varying emitter widths (junction depths) and (c) $V_{O C}$ and $J_{S C}$ plotted for a range of minority carrier diffusion lengths. Reproduced with permission. ${ }^{[3]}$ Copyright 2011, American Institute of Physics.

\subsubsection{Pillar designs for optimal light absorption}

Already by the human eye, it is visible that arrays of silicon micropillars absorb more light than a flat sample, because the reflectivity decreases significantly. Most of the research in the direction of optimized pillar arrays has been performed on pillars with feature sizes in the range of the wavelength of light, because such pillars have long optical paths for efficient light absorption and short carrier transit times. For these nanopillars, the effects of pillar morphology ${ }^{[9]}$ and dimensions such as diameter, length ${ }^{[8,10,11]}$ and pitch $^{[12]}$ on the absorption properties have been investigated. For example, arrays of amorphous silicon nanopillars and -cones were simulated and their reflectivity was compared with a flat thin film of amorphous silicon. ${ }^{[13]}$ The nanopillars and cones had a diameter of $300 \mathrm{~nm}$, where the cones had a gradual decrease in diameter toward their tops, down to $20 \mathrm{~nm}$. Such pillars and cones were also fabricated and comparison of experimental with the simulated data showed that the reflectivity of the thin film is significantly higher than that of the nanopillar samples and the difference is even stronger for the nanocones.

One of the most important factors to decrease reflectivity is the ratio between the diameter $(D)$ and the pitch $(P)$ of the pillars. ${ }^{[6,14,15]}$ For any given height of a pillar, it is claimed that the $D / P$ ratio should be between 0.5 and 0.8 , where the latter shows the highest efficiency. ${ }^{[14,15]}$ The overall efficiency can be increased by increasing the height of the pillars, in which case the feasibility of fabrication of such long pillars seems to be the main limiting factor. 
Furthermore, for a given $D / P$ ratio, a thinner diameter - within a range of 270 to $380 \mathrm{~nm}$ - and smaller pitch is expected to result in a higher efficiency. ${ }^{[14]}$ This trend was not in agreement with other simulations, in which an optimum of 2 micron for the pillar diameter was found. ${ }^{[15]}$

In all studies discussed above, symmetry was always present in the design of the array of pillars, i.e. one set of pillar dimensions was used and the pillars were placed in ordered arrays (hexagonal, triangular, square etc.). However, order is not necessarily the optimal configuration. ${ }^{[16]}$ By reducing the diameter of half of the pillars, the authors were able to increase the absorbance of arrays with two different pillar radii. In another example, it was proposed that random, non-ordered pillar arrays have advantages over ordered structures, such as enhanced absorption for arrays with low areal packing fractions. ${ }^{[17]}$

Summarizing, although many simulation studies aim at the prediction of the effect of individual device design variables, such as pillar diameter and height, array packing density, doping profile and junction depth, there is no overall and consistent picture of the optimal device design for photovoltaic conversion. It would be beneficial to the field to have a generalized framework of simulation, with a fixed set of parameters, to be used for validation of pillar-based designs and prediction of possible limitations of such designs. Since the performance of silicon pillar arrays depends in a highly convoluted manner on design aspects such as pillar dimensions, pitch, doping level and junction depth, simulations of the combined effects of these parameters could help predict which systems are of potential interest for practical application. In addition, such efforts could reveal if there is a clear relationship between the different design parameters, for example, whether the pillar shape would influence the optimal configuration of a pillar array. At the moment, there is no clear indication whether the nano or micron scale is more suited, neither for solar cells nor for solar-to-fuel applications, and sometimes research involves a combination of both micro- and nanoscale structures. ${ }^{[18,19]}$ In addition, it is not yet clear what is the effect of the many different fabrication and doping methods. This can potentially cause differences in terms of the quality of the 
silicon, resulting in differences in, for example, charge carrier density, diffusion lengths and defect levels.

\subsection{Fabrication of silicon nano/micropillars}

There are many ways to produce arrays of nano- and micro-sized silicon pillars, and in this section, an overview is given of the main techniques that have been employed, namely dry etching, wet etching, and vapor-liquid-solid growth.

\subsubsection{Dry etching}

In general, prior to dry etching, a pattern with the desired dimensions is created in a protective layer on the silicon substrate, which acts as an etch mask. Most commonly, standard UV photolithography is used to transfer the desired mask pattern into a layer of photoresist, mostly followed by a postbake of the photoresist. ${ }^{[20-24]}$ Dry etching of the mask pattern in silicon, by a combination of bombarding its surface with reactive ions and the chemical etching of reactive species (i.e. radicals), leads to the desired structures. The most common form of dry etching is (deep) reactive ion etching (D)RIE, which uses chemically reactive plasmas (e.g. $\mathrm{SF}_{6}, \mathrm{O}_{2}, \mathrm{C}_{4} \mathrm{~F}_{8}$ ) in a vacuum chamber as a source to dissolve exposed silicon. This can be done either in a continuous flow of reactive gases (cryogenic etching) or with alternating etching and passivation gases (Bosch process, shown in Figure 2.2). ${ }^{[25]}$

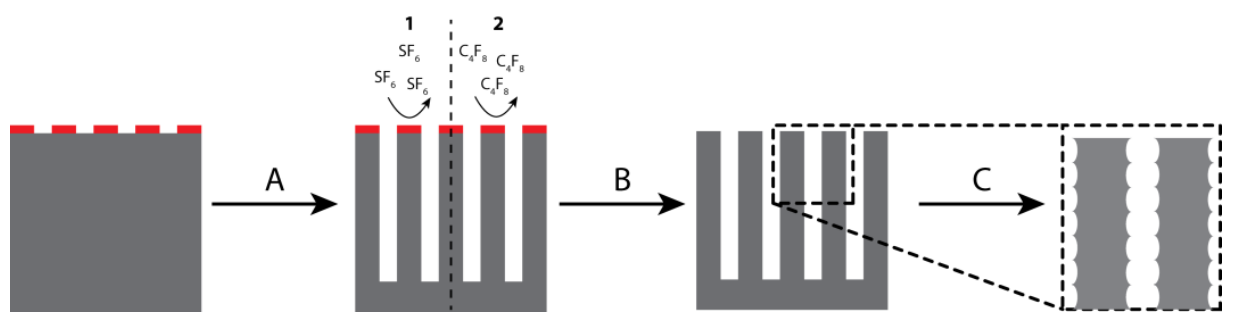

Figure 2.2: Typical deep reactive ion etching scheme for the Bosch process. (A) After definition of a mask on the silicon surface, etching and passivating gasses are introduced alternatingly. (B) These processes are cycled until the desired height has been achieved, after which the patterned mask and fluorocarbon contamination are removed. (C) Zoom-in of the pillars, showing the typical sidewall scalloping, resulting from the cyclic Bosch process. 
Although dry etching is a well-known technique, various factors (e.g. aspect ratio, total area to be etched and RIE lag) affect the realization of high aspect ratio structures, as described in a review by Rangelow et al. ${ }^{[26]}$ If the DRIE process has been tuned correctly, almost no under-etch of the mask will occur, and the pillar cross-section will be the same as the pattern in the etching mask. The structures etched into silicon typically have a diameter of at least a few microns in the case of UV lithography, whereas the heights can go up to several tens of microns. ${ }^{[27]}$ The method also allows pillars with sub-micron diameters, the limiting factor being the scalloping due to the cyclic steps in the Bosch process. For sub-micron features, deep-UV lithography (Figure 2.3) (or another method described below) is needed. ${ }^{[28]}$

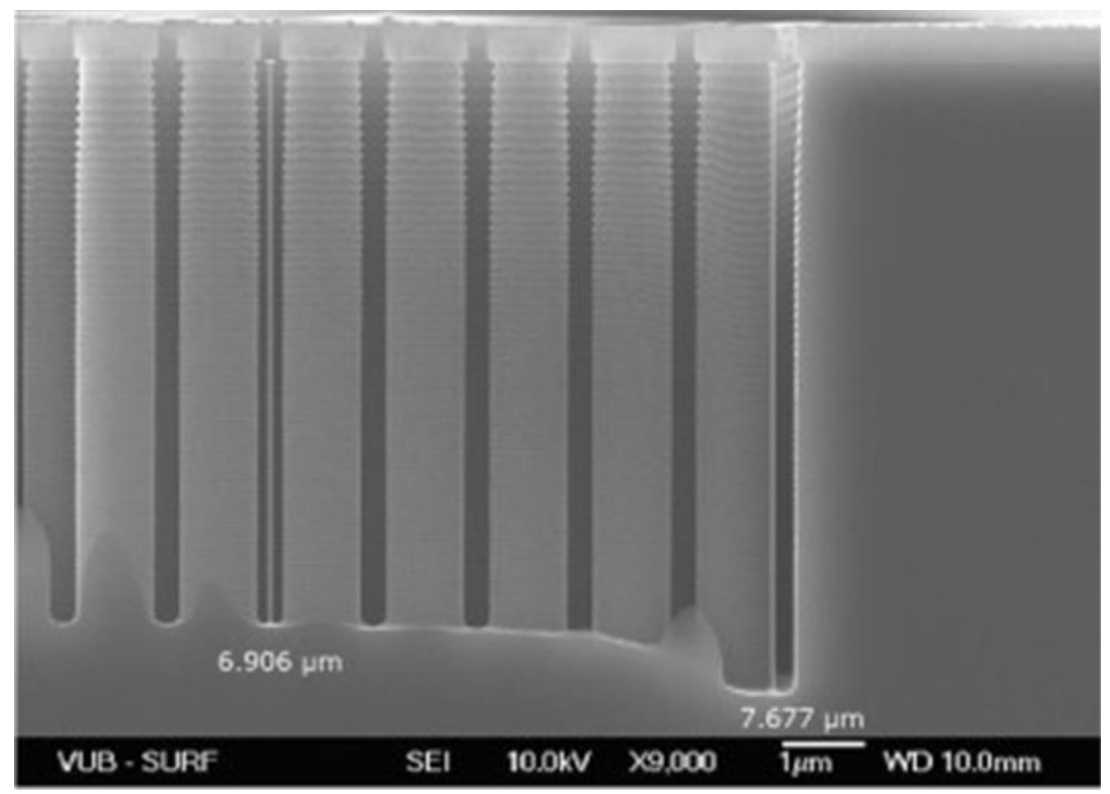

Figure 2.3: Side view scanning electron microscopy (SEM) image of silicon micropillars etched with DRIE, using a mask made with deep UV lithography. Scallops are visible at the top of the pillar, which is an artifact from the Bosch process. Reproduced with permission. ${ }^{[28]}$ Copyright 2012 , Elsevier.

Instead of photoresist, other materials can also function as masking layer, such as a "hard mask" of silicon nitride or silicon oxide, into which a pattern is created by photolithography and etching, before the sample is exposed to DRIE. ${ }^{[29]}$ The use of a hard mask avoids the problem of flowing of the resist, 
which can occur at longer process times or higher process temperatures, conditions needed to obtain higher aspect ratios. Alumina is an excellent alternative hard mask layer, which exhibits a high etch selectivity, allowing longer etching times and thereby longer pillars, with heights above $150 \mu \mathrm{m} .{ }^{[30]}$

In order to fabricate silicon pillars with diameters in the range of nanometers, other lithography techniques have to be applied, such as laser interference lithography $(\mathrm{LIL})^{[31]}$ or nanosphere lithography (NSL). ${ }^{[32,33]}$ For example, a close-packed monolayer of polystyrene spheres drop-casted on a layer of $\mathrm{SiO}_{2}$ followed by DRIE enabled the fabrication of nanopillars. ${ }^{[32,33]}$ The size of the polystyrene particles was adjusted by oxygen plasma, such that a non-closepacked monolayer became available for etching (Figure 2.4). Another example of the use of particles as a mask employs the self-assembly of cesium chloride particles. ${ }^{[34]}$ After a film of these particles was deposited on the silicon substrate and the temperature was lowered, the particles self-assembled by absorbing water from the air environment. A wide variety of pillar diameters was obtained ( $50 \mathrm{~nm}-1 \mu \mathrm{m})$, however, the pillar height was limited by the etch selectivity between the particles and silicon, resulting in rather low aspect ratios of 2-6.

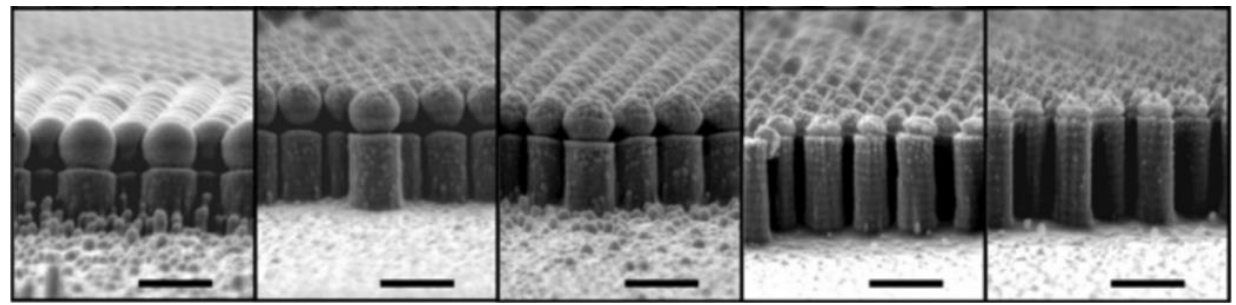

Figure 2.4: Side view SEM images of nanospheres used as an etch mask using DRIE of silicon, after different times of oxygen plasma exposure prior to etching to reduce the size of the nanospheres. Left image is not exposed to oxygen plasma, followed by $30,60,90$ and 120s (right) of oxygen plasma. Scale bars represent $750 \mathrm{~nm}$. Reproduced with permission. ${ }^{[33]}$ Copyright 2006, IOP Publishing.

To fabricate wafer scale arrays of pillars, nanoimprint lithography is a promising technique for the formation of the mask layer. ${ }^{[35]}$ In short, a thermoplastic or a photocurable polymer is patterned on a substrate by an imprint mold, and this layer can act as a mask during DRIE. The feature sizes 
that can be obtained with this technique reach the sub-10 nanometer range. ${ }^{[36]}$ Using the combination of NIL and DRIE, silicon pillars with an aspect ratio of 60 and a diameter of $50 \mathrm{~nm}$ have been made on wafer scale. ${ }^{[35]}$

Another method to create large areas of silicon pillars is maskless etching, which uses a technique called black silicon etching. ${ }^{[37,38]}$ By tuning the settings of the etch process, small contaminations on the wafer, i.e. deliberately created by-products or reaction intermediates from the etch process, can act as nanomasks on the silicon surface, resulting in the formation of spikes. ${ }^{[38]}$ As the technique does not require a mask, wafer scale etching is easily achieved, and the fabrication of silicon pillars with diameters between $50-80 \mathrm{~nm}$, with heights up to $1600 \mathrm{~nm}$, has been shown. ${ }^{[37]}$ Note that these structures are randomly positioned, and pillar diameter and pitch cannot be defined by design. Note also that by the nature of the black silicon process, the pillars will have a small but notable taper.

\subsubsection{Wet etching}

In contrast to dry etching, wet etching does not require a reaction chamber under vacuum, but is instead performed at atmospheric pressure in a liquid. This increases the possible throughput significantly, as several wafers can be etched at the same time, without any preloading requirement. Two types of wet etching methods are frequently applied for the fabrication of silicon pillars, namely electrochemical wet etching and metal-assisted chemical etching (MACE). Both methods are schematically illustrated in Figure 2.5. 

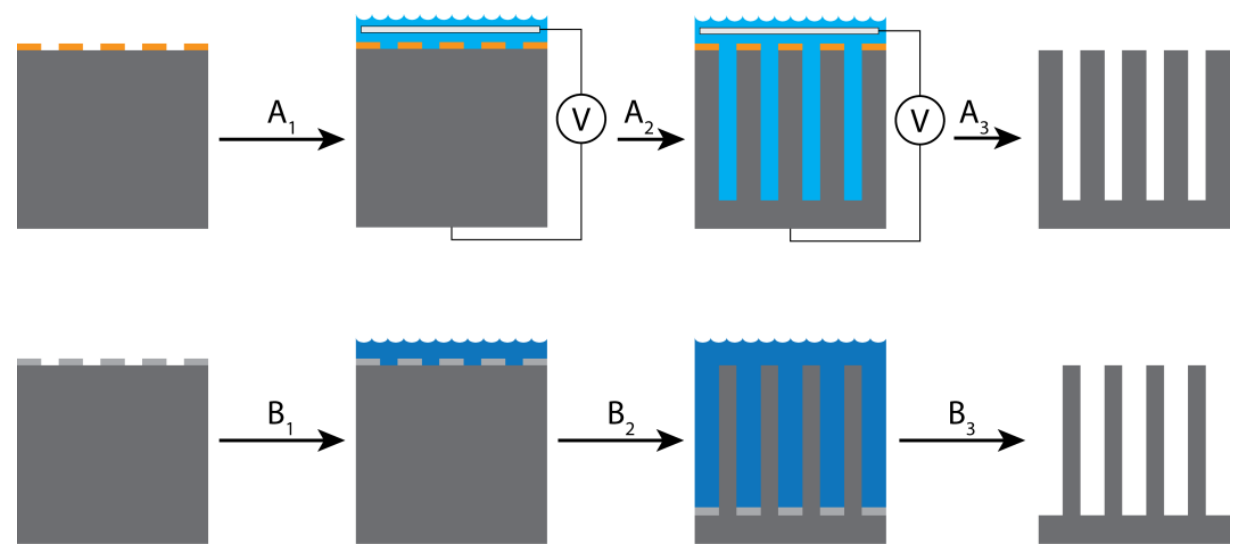

Figure 2.5: Schematic representation of typical electrochemical etching $(A)$ and MACE $(B)$ processes. (A1) A hard mask (e.g. $\mathrm{SiO}_{2}$ or $\mathrm{SiN}_{\mathrm{x}}$ ) is patterned on the silicon surface, and the substrate is placed in an HF solution, connected between two electrodes. (A2) An anodic bias is applied and the exposed silicon is etched down. (A3) The sample is removed from the solution, followed by removal of the resist mask. (B1) A metal mask (typically silver) is patterned on the surface and placed in an HF solution. (B2) The metal film is etched into the silicon surface, leaving the non-patterned silicon untouched.

(B3) The silicon sample is removed from the solution and the metal masked is stripped.

During the process of electrochemical etching, an electrolyte, in combination with an anode and cathode, creates a charged double layer near the surface of the silicon, which results in the creation of nanostructures. ${ }^{[39]}$ The shapes of these structures can either be defined by the shape of a physical object that is brought in close proximity of the substrate, or by using a masking layer, which can be deposited by various methods. ${ }^{[39,40]}$ Using this technique, many complex shapes have been created, when the proper masking steps were used. ${ }^{[41,42]}$ For example, in an comprehensive study on electrochemical etching, Bassu et al. were able to fabricate a MEMS device, with high-aspect ratio $(100)$ comb fingers suspended by high-aspect ratio folded springs. ${ }^{[43]}$

Metal assisted chemical etching (MACE) is a process that starts with the deposition of metallic nanoparticles (usually silver) on the surface of silicon, followed by the electroless etching in an aqueous mixture of $\mathrm{H}_{2} \mathrm{O}_{2}$ and $\mathrm{HF}$. ${ }^{[4,45]}$ It is proposed that the silver particles sink into the silicon, thus etching the silicon under the silver nanoparticles, whereas the uncovered silicon remains intact. For a more detailed discussion about the possible mechanism for 
MACE, see the article by Geyer et al. ${ }^{[46]}$ Typically, the process results in the formation of silicon nanopillars with diameters from $<10 \mathrm{~nm}$ up to a few hundred $\mathrm{nm}$, and with heights depending on the etching time, ranging from a few $\mu \mathrm{m}$ up to $20 \mu \mathrm{m} .^{[2,47-49]}$ Peng et al. investigated the etching direction of both gold and silver particles, and concluded that this direction is highly uniform, does not depend on the dopant type and level of the substrate, and preferentially occurs along the (100) orientation of crystalline silicon (Figure 2.6). ${ }^{[50]}$ Instead of electroless deposition of metal particles, it is also possible to deposit a thin metal film, which is patterned or annealed to create nanoparticles. An example can be found in the work of Huang et al., ${ }^{[51]}$ where the previously described masking method using polystyrene beads was applied to control the silver nanoparticle size before MACE.
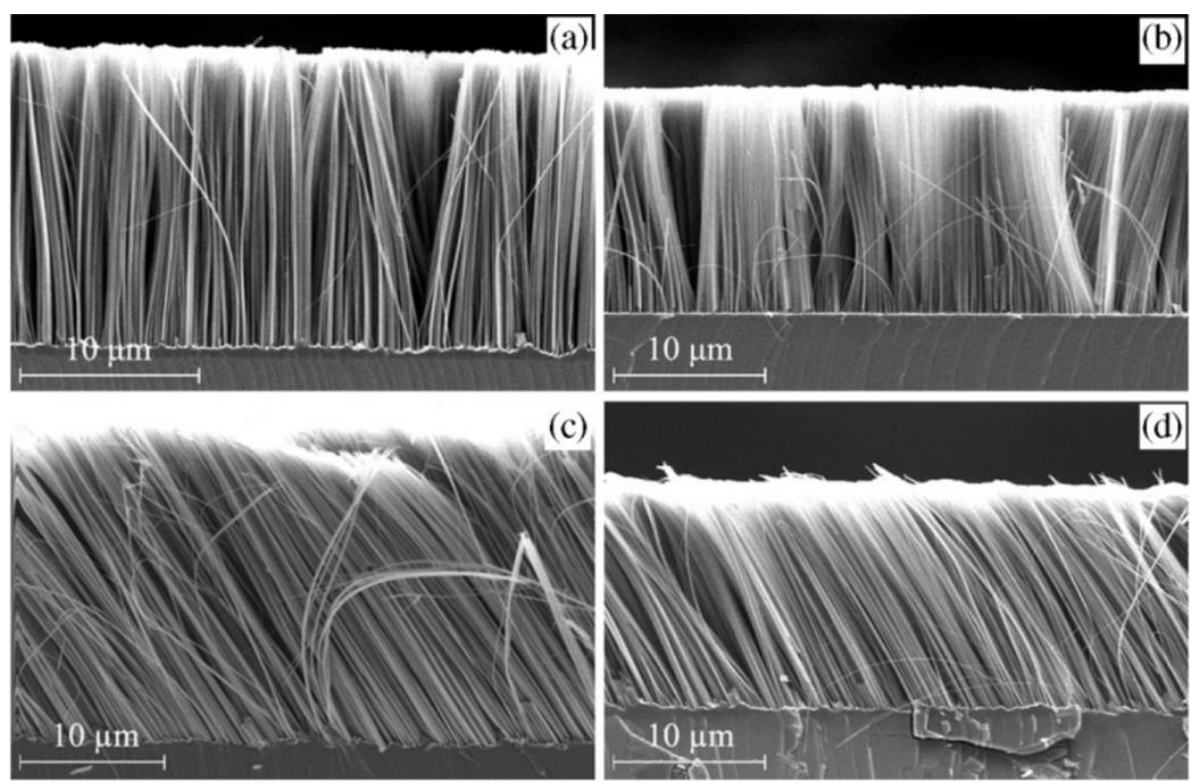

Figure 2.6: Cross section SEM images of silicon substrates coated with Ag particles, followed by 30 min MACE process in $\mathrm{HF} / \mathrm{H}_{2} \mathrm{O}_{2}$ on: (a) p-type silicon (100), (b) p-type silicon (111), (c) p-type silicon (110) and (d) n-type silicon (113) substrates. Reproduced with permission. ${ }^{[50]}$ Copyright 2008, Wiley- $\mathrm{VCH}$.

\subsubsection{Vapor-liquid-solid growth}

The most popular bottom-up method for the growth of silicon pillars is the so-called vapor-liquid-solid (VLS) growth, which is schematically shown in 
Figure 2.7. Already in 1964, this technique was discovered and a process was reported in which a small gold particle was used to grow a single silicon pillar from the vapor phase. ${ }^{[52]}$ This particle was heated up to $950{ }^{\circ} \mathrm{C}$, forming a gold-silicon alloy, and by supplying hydrogen and silicon tetrachloride, the liquid alloy acted as a sink for the silicon atoms. Increase of the reaction time led to saturation of the alloy, and silicon froze out below the liquid particle. The here described process uses chemical vapor deposition (CVD) to supply silicon for the growth, but many other techniques can also be used, such as laser ablation, electron beam evaporation, and physical transport as described in a review of Barth et al. ${ }^{[53]}$

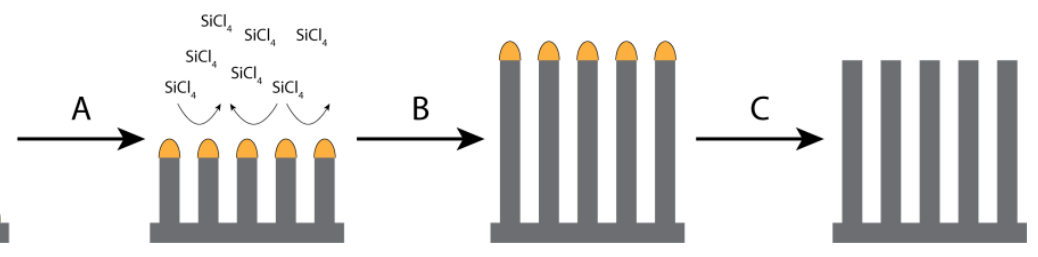

Figure 2.7: Schematic illustration of a typical VLS silicon pillar growth process. (A) After a metal pattern is defined, a silicon gas is introduced and dissolved in the metal particle, which initiates pillar growth. (B) The time and flow of the gas is used to control the height of the pillars. (C) After the process, the metal particles are removed from the top of the pillars.

In the last decade, many improvements of the VLS process were reported. For example, the possibility to grow small arrays of pillars, instead of single pillars, was shown by the use of electron beam lithography and metal lift-off. ${ }^{[54]}$ For this method surface migration of the metal catalyst (gold in this case) determines the height, shape and sidewall properties of the silicon pillars. ${ }^{[55]}$ To circumvent this issue and to obtain smooth and arbitrarily long pillars in a large array, the gold diffusion has to be controlled. As a result, templates were used to control the catalyst diffusion and pillar growth, and this resulted in large arrays of VLS grown pillars. ${ }^{[56]}$ The group of Atwater introduced a $300 \mathrm{~nm}$ buffer oxide layer as a barrier between individual metal particles, to avoid the use of a template and to prevent agglomeration of the particles at the same time. ${ }^{[57,58]}$ By doing this, they were able to fabricate large areas $\left(>1 \mathrm{~cm}^{2}\right)$ of silicon pillar arrays without the use of a template (Figure 2.8). 


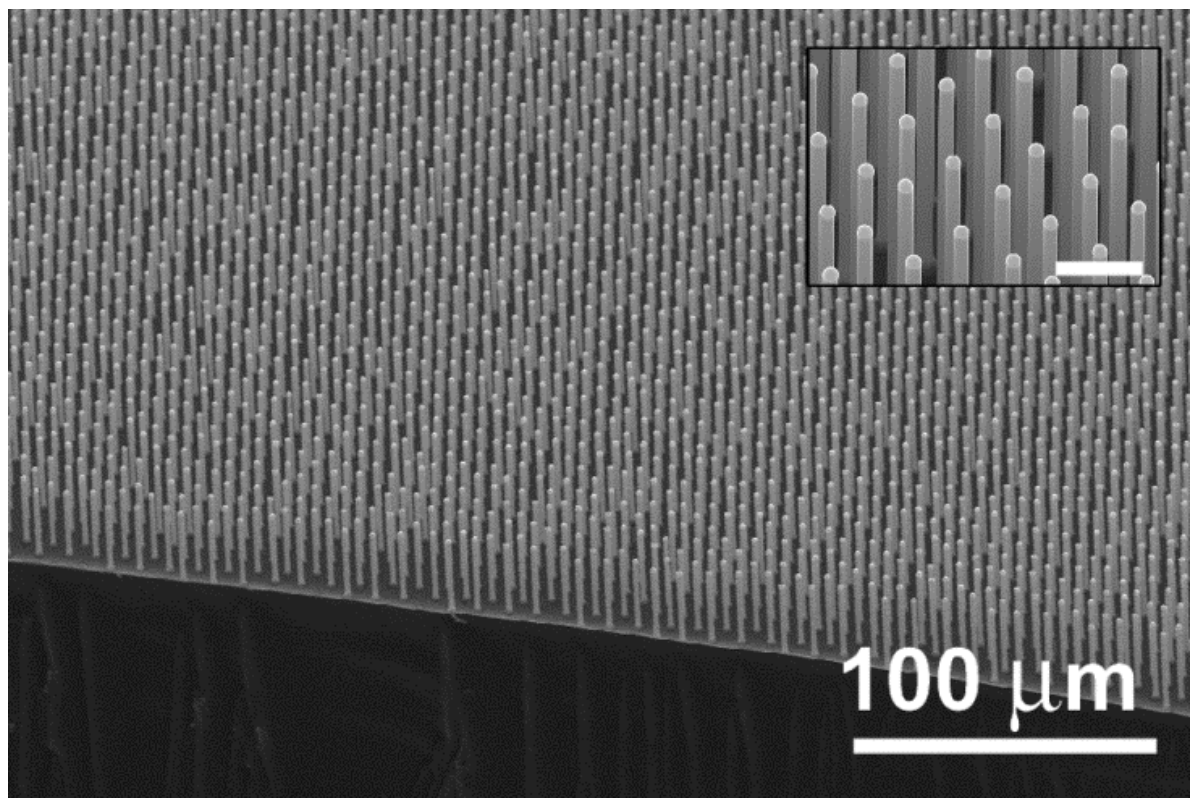

Figure 2.8: Tilted SEM image of a large-scale $\left(>1 \mathrm{~cm}^{2}\right)$ Cu-catalyzed VLS grown silicon micropillar array. Inset shows a zoom in of several pillars (scale bar is $10 \mu \mathrm{m}$ ). Reproduced with permission. ${ }^{[57]}$ Copyright 2007, American Institute of Physics.

\subsubsection{Comparison of fabrication techniques}

As a summary, Table 2.1 gives a selective overview of the reported possible pillar dimensions. The numbers given should not be seen as a real limitation of the fabrication methods, but as an indication in which range the technique is commonly used.

Table 2.1: Overview of different fabrication techniques, used for the fabrication of silicon nano- and micropillars.

\begin{tabular}{lcccc}
\hline \multicolumn{1}{c}{ Etch technique } & $\begin{array}{c}\text { Etch } \\
\text { type }\end{array}$ & $\begin{array}{c}\text { Height } \\
(\boldsymbol{\mu m})\end{array}$ & $\begin{array}{c}\text { Diameter } \\
(\mathbf{n m})\end{array}$ & $\begin{array}{c}\text { Wall-to-wall } \\
\text { distance }(\mathbf{n m})\end{array}$ \\
\hline DRIE \& UV lithography ${ }^{[20-23,25]}$ & Dry etch & $1-150$ & $>1000$ & $>1000$ \\
DRIE \& Deep-UV lithography ${ }^{[2,59]}$ & Dry etch & $1-150$ & $>250$ & $>250$ \\
DRIE \& Nanosphere lithography ${ }^{[32-34]}$ & Dry etch & $<10$ & $50-500$ & $50-500$ \\
DRIE \& Nanoimprint lithography ${ }^{[35,36]}$ & Dry etch & $<5$ & $10-100$ & $>100$ \\
Electrochemical wet etching $^{[40,41]}$ & Wet etch & $<25$ & $500-2000$ & $>1000$ \\
Metal assisted chemical etching $^{[49,50,60]}$ & Wet etch & $<20$ & 100 & $<10$ \\
Vapor-liquid-solid growth $^{[54-58]}$ & Growth & $1-100$ & $50-1500$ & $50-5000$ \\
\hline
\end{tabular}




\subsection{Doping of silicon}

Silicon can be doped using several techniques yielding greatly varying surface concentrations, junction depths and doping profiles. This section describes processes utilized for the $p$ - and n-type doping of silicon, on either flat or structured surfaces. We will discuss whether the techniques have been, or potentially could be used for doping of micro/nanopillars. Although there are several elements ( $\mathrm{B}, \mathrm{P}, \mathrm{Sb}, \mathrm{As}, \mathrm{Ga})$ that can be used for the introduction of $\mathrm{p}$ - and $\mathrm{n}$-type dopants in silicon, only boron and phosphorus will be discussed here as they are most commonly used. The standard process for any doping method is divided into two steps: first the dopant atoms are introduced at the surface of the silicon substrate via the formation of a layer (usually an oxide layer), followed by a drive-in step during which dopant diffusion into silicon takes place. Exceptions are ion implantation, where the dopant is injected into the silicon substrate (see 2.4.1), and epitaxial growth, where doping is performed in-situ during silicon layer deposition. ${ }^{[61]}$ The temperature and time control during the drive-in step is important for the final doping profile. When the deposition temperature of the dopant-containing layer is higher than $800^{\circ} \mathrm{C}$, the formation of such layer and the dopant diffusion into silicon occur simultaneously.

\subsubsection{Ion implantation}

During ion implantation, ions are accelerated towards the silicon target, which results in doping. ${ }^{[62]}$ Although recent research shows good reproducibility with respect to the doping dose and profile, this technique requires a thermal annealing step after the doping, to ensure defect healing and dopant activation, because of the damage to the silicon crystal lattice generated by the energetic ions. ${ }^{[63]}$ The directionality of this technique makes ion implantation less suited for radial doping of silicon micro/nanopillars, but it may be an option for the generation of a dopant gradient along the axial direction of pillars. In the latter case, it would be preferred to use implantation in combination with DRIE, in which case the ion implantation can be performed 
before etching the pillars. DRIE of silicon has shown little dependence on the doping level, for moderate dopant levels. ${ }^{[64,65]}$

\subsubsection{Chemical vapor deposition}

Chemical vapor deposition (CVD) is a widely used technique to form layers of dopant oxides on silicon surfaces, which are subsequently used as diffusion sources. In CVD at atmospheric-pressure (APCVD) $)^{[3,66,67]}$ high flow rates (1500-3000 sccm) of reactive gases (e.g. $\mathrm{PH}_{3}$ and $\mathrm{B}_{2} \mathrm{H}_{5}$ ) are supplied, and a conformal layer is formed at elevated temperatures (usually above $900{ }^{\circ} \mathrm{C}$ ). By controlling the ratio between the oxide and dopant flow, the dopant concentration in the oxide layer can be controlled, which in turn determines, along with the anneal time and temperature, the characteristics (junction depth and dopant level and profile) of the doped silicon layer. After the dopant layer has been deposited, the temperature is further increased to the desired drivein temperature to increase the dopant diffusion rate. Owing to the atmospheric pressure condition, this technique can be easily scaled up to large batches of wafers, as shown by Rothhardt et al. ${ }^{[66]}$ In case of doping 150 wafers in an industrial scale furnace, there was no difference in sheet resistance between the wafer positioned near the gas inlet and at the end of the wafer boat.

In order to obtain similar layers as described for APCVD, but at lower temperatures, plasma-enhanced CVD (PECVD) is a suitable option. ${ }^{[68,69]}$ During PECVD, electrons rapidly gain energy through a radio frequency (RF) field and, combined with a reagent gas (e.g. $\mathrm{PH}_{3}$ ), they form highly reactive chemical species that produce the desired layer, already at a temperature of $300{ }^{\circ} \mathrm{C} .{ }^{[70]}$ PECVD can also be used to deposit in-situ doped silicon, by means of which the drive-in step can be omitted, but it yields an amorphous or poly-crystalline layer. ${ }^{[71,72]}$

Hot-wire chemical vapor deposition (HWCVD), also referred to as catalytic CVD, is used for the deposition of mainly inorganic thin films. ${ }^{[73,74]}$ During the deposition under vacuum, a precursor source is heated by a metallic filament to obtain conformal thin films on various substrates, for example 
nanostructured silicon. ${ }^{[75]}$ By combining the precursor with the desired dopant molecules, shallow junctions can be formed, as shown by several different research groups. ${ }^{[76-78]}$ As the dopant layer is deposited on silicon, no additional drive-in step is required. The main advantage of HWCVD is the absence of a plasma, thus obviating the risk of damaging the silicon substrate by bombardment with energetic ions.

Another method to deposit boron or phosphorus containing layers, is low-pressure CVD (LCPVD). The layer thickness and dopant concentration of the grown layer are controlled by the pressure and the gas inlets. For this process, a pressure of typically a few hundred mTorr is often used, corresponding to gas flows that are significantly lower than in the case of APCVD, (i.e. 100-500 sccm). ${ }^{[79,80]}$ Depending on the application different gas mixtures are supplied, for example, $\mathrm{PH}_{3}$ and $\mathrm{SiH}_{4}$ for in situ phosphorus doping of polycrystalline silicon films, yielding a phosphorus doped layer. ${ }^{[81]}$ The LPCVD technique is mainly used to grow in-situ doped polysilicon, which is characterized by the absence of mechanical stress and a low electrical resistivity, however, it can also be used as a dopant source for the doping of single-crystalline silicon, by depositing a dopant containing oxide layer.

\subsubsection{Solid source dotation}

In the case of solid source dotation (SSD), solid wafers of either boron nitride (for p-type doping) or cesium phosphate (for n-type doping) are used to supply the dopant species to the silicon surface. The dopant atoms are transferred by evaporation from the solid source, diffuse to the silicon surface, and are incorporated in-situ in a growing oxide layer. In the case of boron doping, the deposited layer consists of boron oxide, grown during the exposure of boron nitride wafers at elevated temperatures under an oxygen flow. ${ }^{[82]}$ By varying the deposition time, the layer thickness can be varied, however, the maximum solubility of boron in silicon (approximately $10^{20}$ atoms $/ \mathrm{cm}^{3}$, depending on the drive-in temperature ${ }^{[83]}$ ) is already achieved after a 30 min growth step. 
This means that the junction depth is primarily controlled by the time and temperature of the anneal step. Directly after this step, the temperature is further increased for the drive-in step. Using this technique, it is possible to form junctions ranging from a few hundreds of nanometers to several microns. ${ }^{[84]}$ The same procedure can be followed for $n$-type doping of silicon with phosphorus, by using solid cesium phosphate wafers. ${ }^{[85,86]}$

\subsubsection{Monolayer doping}

Monolayer doping (MLD) uses hydrosilylation to chemically attach either boron or phosphorus containing molecules to a hydrogen-terminated silicon surface, that is formed by the wet etching of the native oxide by aqueous fluoride. ${ }^{[87]}$ After the attachment of the molecules, a silicon dioxide capping layer is deposited onto the monolayer, and subsequently a rapid thermal annealing (RTA) step is performed to drive in the dopant molecules into the silicon. The final junction depth depends on the amount of dopant atoms in the monolayer, and the RTA time and temperature. In 2011, a variation of this method was published achieving the local doping of areas of a silicon substrate. ${ }^{[8]}$ By combining MLD with nanoimprint lithography and reactive ion etching, a pattern was made in the dopant layer on the silicon, which was then analyzed with SIMS to evidence the method and function. Monolayer doping has many advantages, such as the variety of reactions and molecules that can be used to form the monolayer on silicon and the possibility to form ultra-shallow junctions, in the range of a few nanometers. ${ }^{[89-91]}$ As shown in Figure 2.9, the group of Javey managed to scale-up the MLD technique to wafer scale, enabling the possibility of large-volume production. ${ }^{\left[{ }^{92]}\right.}$ 


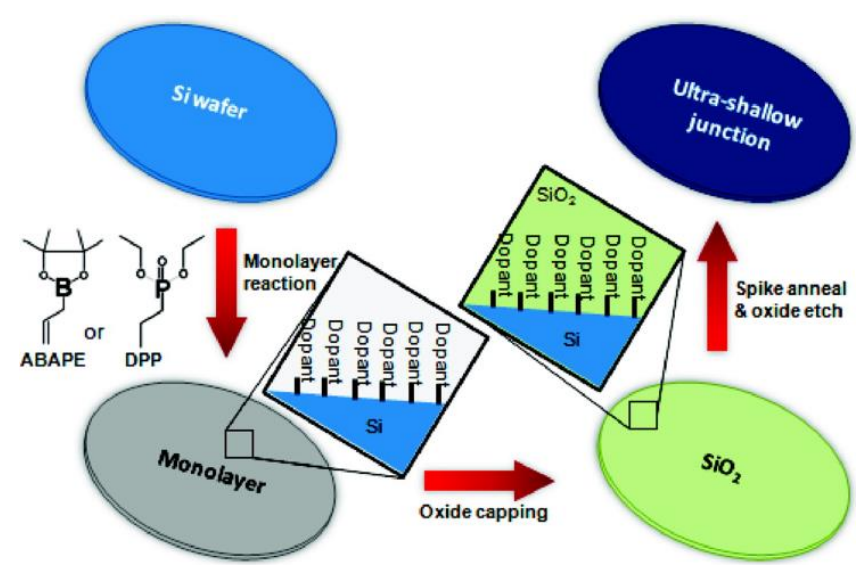

Figure 2.9: Schematic representation of a full wafer scale MLD process for either boron or phosphorus doping. Reproduced with permission. ${ }^{[92]}$ Copyright 2009, American Chemical Society.

\subsubsection{Spin-on dopant}

Doped silicon layers can also be fabricated by using a spin-on-dopant (SOD). With this method, a spin-on-glass (SOG) solution containing either boron of phosphorus, is spin-coated on a silicon substrate. After a short low-temperature drying step, the coated wafer is heated up to the drive-in temperature, usually in the range of $850-1100{ }^{\circ} \mathrm{C}$, at which dopant diffusion into silicon occurs. ${ }^{[93,94]}$ Different concentrations of dopants in SOG solutions can be obtained and used to tune the doping profile and junction depth. Afterwards, the glassy layer is removed with a buffered hydrogen fluoride solution. Since no vacuum is required to form the SOD layer and the spinning of the SOD layer only takes about $30 \mathrm{~s}$, the technique has a large advantage in terms of high-throughput fabrication of $\mathrm{p} / \mathrm{n}$ junctions. The technique is suitable for both ultra-shallow $(12 \mathrm{~nm}$ ) and deep junctions (several micrometers), and is a relatively easy and fast alternative to the previously described doping methods. ${ }^{[18,93]}$ The versatility of SOD is evidenced by literature examples, in which SOG is used in combination with SODs to selectivity dope certain areas on a silicon wafer, or where organic polymers are used (instead of inorganic) that are burnt away during the diffusion step. ${ }^{[27,95]}$ 
In another example, an axial $\mathrm{p} / \mathrm{n}$ junction was created by filling the areas between silicon pillars in an array with SOG, followed by an RIE step to access the top part of these pillars, and subsequent deposition of an SOD layer for the diffusion of dopants in the top part. ${ }^{[96]}$

\subsubsection{Proximity doping}

To enhance the control over silicon doping, already in 1994 proximity doping was introduced in combination with SOD. ${ }^{[97]}$ The idea behind proximity doping is the use of a dummy wafer, onto which a finite SOD layer is previously applied, that is brought in close proximity $(\sim 400 \mu \mathrm{m})$ of the target wafer. By controlling the distance between dummy and target wafer during the drive-in step, the doping characteristics of the target wafer can be altered in a controlled way. The junction depth is determined by the concentration of the dopant on the dummy wafer, and the time and temperature used during the transfer step. More recently, the combination of SOD with proximity doping has been used to dope silicon pillars to ensure a homogeneous distribution over the height over the pillar, and control over the surface concentration between $10^{18}-10^{20}$ atoms $/ \mathrm{cm}^{3}$ has been shown. ${ }^{[18,98]}$ The use of proximity doping is not limited to SOD samples, as any substrate with a dopant layer can be used. The proximity principle has been shown to work as well in combination with MLD, by forming a dopant containing monolayer on a donor substrate and using an RTA step to dope both the target and donor substrate. ${ }^{[99,100]}$ By repeating this procedure multiple times, higher doping concentrations were achieved for the target substrates, as well as deeper $(>100 \mathrm{~nm})$ junctions.

\subsubsection{Comparison of methods for doping silicon}

An overview of all doping techniques is given in Table 2.2. Besides the requirements of a vacuum for the method and necessity of a drive-in step, it is indicated whether the method is suited for structured surfaces and which junction depth can be realized. The junction depth range only gives an indication, other values may be possible by tuning of the doping technique. 
Table 2.2: Overview of different doping techniques, used for the fabrication of silicon $\mathrm{p} / \mathrm{n}$ junction (boron and phosphorus).

\begin{tabular}{|c|c|c|c|c|}
\hline Doping technique & Vacuum? & $\begin{array}{c}\text { Drive-in } \\
\text { step? }\end{array}$ & $\begin{array}{c}\text { Junction } \\
\text { depth }(\mathrm{nm})\end{array}$ & $\begin{array}{c}\text { Radial } \\
\text { junction? }\end{array}$ \\
\hline Ion implantation ${ }^{[62,63,101,102]}$ & Yes & $\mathrm{No}^{1}$ & $<1000$ & No \\
\hline Atmospheric-pressure $C V D^{[3,66,103]}$ & No & Yes & $100-3000$ & Yes \\
\hline Plasma-enhanced CVD ${ }^{[68,70,72]}$ & Yes & Yes & $100-3000$ & Yes \\
\hline Hot-wire $C V D^{[77,78]}$ & Yes & No & $<100^{2}$ & Yes \\
\hline Low-pressure $C V D^{[79,80]}$ & Yes & Yes & $100-1000$ & Yes \\
\hline Solid source dotation ${ }^{[82,85,86]}$ & No & Yes & $100-3000$ & Yes \\
\hline Monolayer doping ${ }^{[87,88,91]}$ & No & Yes & $<100$ & Yes \\
\hline Spin-on dopant ${ }^{[27,93-95]}$ & No & Yes & $10-3000$ & Yes \\
\hline Proximity doping ${ }^{[97-99]}$ & No & Yes & $10-1000$ & Yes \\
\hline
\end{tabular}

1) Although a drive-in step is not necessary for silicon doping, a high-temperature annealing step is needed to repair crystal damage.

2) The junction is realized as a layer, meaning that the junction depth is limited to the layer thickness that can be deposited.

\subsection{Junction analysis}

In this section, the analysis of the formed $\mathrm{p} / \mathrm{n}$ junction in silicon is discussed. It gives an overview of the analysis on flat and structured surfaces, as well as $J V$ measurements on silicon $\mathrm{p} / \mathrm{n}$ junctions.

\subsubsection{Flat surfaces}

To verify the presence of the introduced dopants, several methods can be used. The most common technique is secondary ion mass spectrometry (SIMS). By sputtering off the top of the doped silicon layer by layer with an ion beam, and measuring the mass and charge of the ions coming off the surface, detailed information about the elemental composition can be obtained as a function of the depth. By comparing the obtained values to a standard, the information is translated into quantities and thus concentration. This technique is suitable for both deep and ultra-shallow junctions, making it useful for almost all doping techniques. ${ }^{[103,104]}$ For example, several groups used SIMS to confirm the formation of an ultra-shallow junction with MLD with a junction depth of about $5 \mathrm{~nm}$ (Figure 2.10). ${ }^{[89,92]}$ 


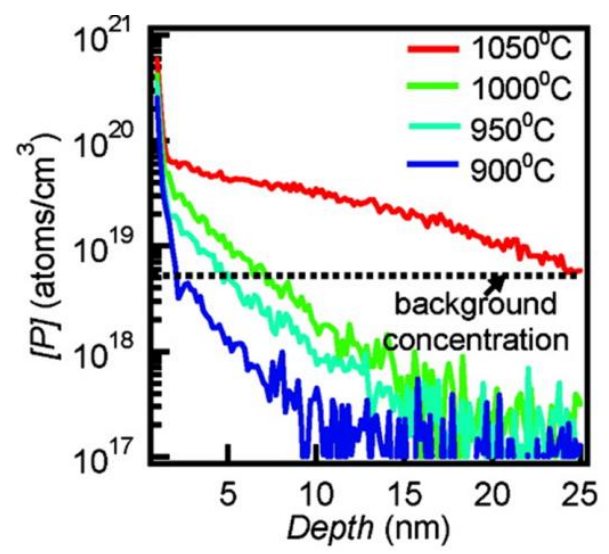

Figure 2.10: SIMS measurements on silicon samples doped with phosphorus using the MLD technique, at various spike anneal temperatures. Reproduced with permission. ${ }^{[92]}$ Copyright 2009, American Chemical Society.

Although SIMS gives information about the surface concentration and the doping profile, the data does not give an exact value for the junction depth itself. This is due to the limitation of SIMS, which can only detect positive or negative ions in a single measurement. Furthermore, the detection limit of SIMS is often in the range of the order of the base dopant concentration of the silicon, meaning that only trends in the doping profile are visible, but an accurate value for the junction depth cannot be determined.

Junction depths can be accurately determined by spreading resistance profiling (SRP), in which the resistivity of doped samples is analyzed as a function of the depth, by measuring on a beveled surface $\left(+/-3^{\circ}\right)$ with two probes. ${ }^{[105]}$ With this technique, it is possible to quantify thin junctions in silicon, for example a $100 \mathrm{~nm}$ junction formed by MLD on a p-type wafer. ${ }^{[106]}$

A simple method to analyze the junction depth is ball grooving and staining. ${ }^{[107]}$ This method consists of the formation of a groove in the doped flat silicon substrate by milling for a few seconds with a diamond-slurry stainless steel ball. This creates a very shallow groove, the depth of which should be larger than the junction depth. Once both the doped layer and the base silicon wafer are exposed, a staining solution is applied to create a contrast difference between the two areas. Suitable staining solutions are aqueous hydrofluoric 
acid with a few droplets of nitric acid, or aqueous chromium trioxide diluted with hydrofluoric acid. ${ }^{[107,108]} \mathrm{A}$ clear contrast is visible after staining because the silicon etching rate depends on the dopant type and concentration. By measuring the radius of the two differently colored areas, combined with the radius of the ball used for grooving, the junction depth can be expressed as: ${ }^{[107]}$

$$
x_{j}=\frac{a^{2}-b^{2}}{2 * R}
$$

Where $x_{j}$ is the junction depth, $R$ is the radius of the ball, $a$ is the radius of outer ring and $b$ is the radius of the inner ring. On its own, ball grooving only gives a value for the junction depth, and no information regarding the doping concentration and profile. However, when combined with, for example sheet resistance measurements or SRP, it can function as a quick verification of the junction depth and doping level.

The sheet resistance is directly related to the surface concentration of the $p / n$ junction, and is expressed in $\Omega / \mathrm{sq}$. A typical sheet resistance measurement involves the use of a four-point probe, where a fixed current is applied to two probes and the potential between the other two probes is measured. In case the doping level is uniform over the whole doping thickness, the resistivity of the wafer can be directly calculated using the junction depth. Unfortunately, this is not the case for most doping techniques, as these result in layers doped in a gradient fashion, meaning that the dopant concentration decreases from the surface to the junction. Yet, the measurement can still provide useful information, especially when comparing different doping settings of the same technique, as the sheet resistance will decrease with increasing surface concentration. ${ }^{[63,68]}$ Similar to ball grooving and staining, the combination of sheet resistance measurements with SIMS results in a good method for verification of the process settings. For example, Hoex et al. showed a set of sheet resistance measurements, verified with SIMS (Figure 2.11), to calibrate their doping settings of the PECVD technique. ${ }^{[109]}$ 


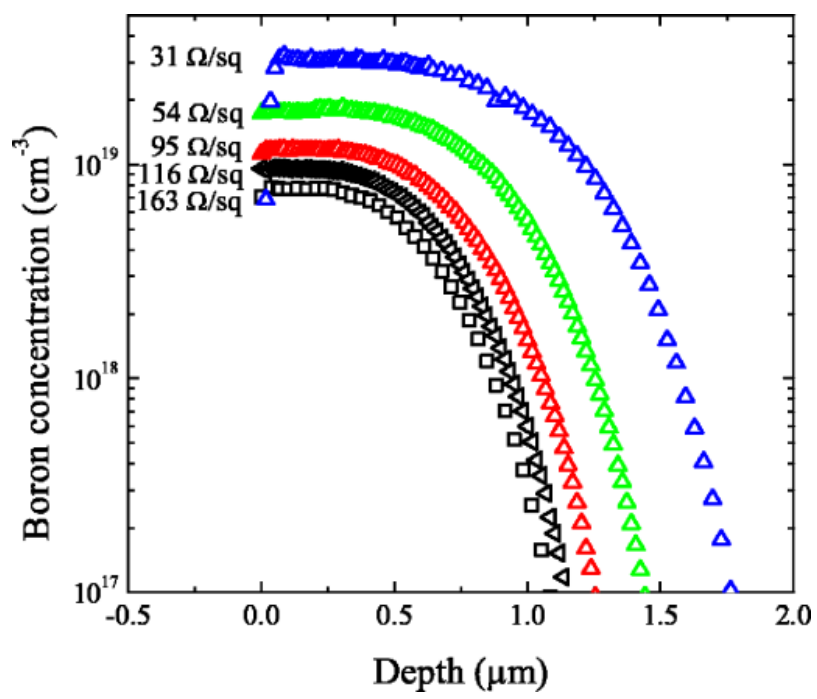

Figure 2.11: Sheet resistance measurements of various boron doped silicon wafers, and their corresponding SIMS profiles; samples were doped at temperatures in the range of $895-1010{ }^{\circ} \mathrm{C}$. Reproduced with permission. ${ }^{[109]}$ Copyright 2007, American Institute of Physics.

\subsubsection{Structured surfaces}

Although the techniques described in section 2.5.1 (or their combination) will result in a good estimation of the junction depth, they can only be performed on flat surfaces. For determining the presence of a junction in a pillar, characterization may be carried out on flat dummy wafers added in the same doping run as the pillared substrates, or better even on a doped flat area adjacent to the pillar array on the same substrate. This however does not give information about the homogeneity of the doping along the pillar height, and in fact, actual junction analysis on structured surfaces is quite rare. For instance, Guo et al. used SIMS measurements to analyze substrates doped with SOD, on both flat and on micro-sized pillar structures $(4 \mu \mathrm{m}$ height, $3 \mu \mathrm{m}$ diameter, Figure 2.12). ${ }^{[22]}$ These SIMS data show an increase of the total doping dose of the structured sample, but do not give an indication whether the doping is homogeneously distributed along the pillar height. 

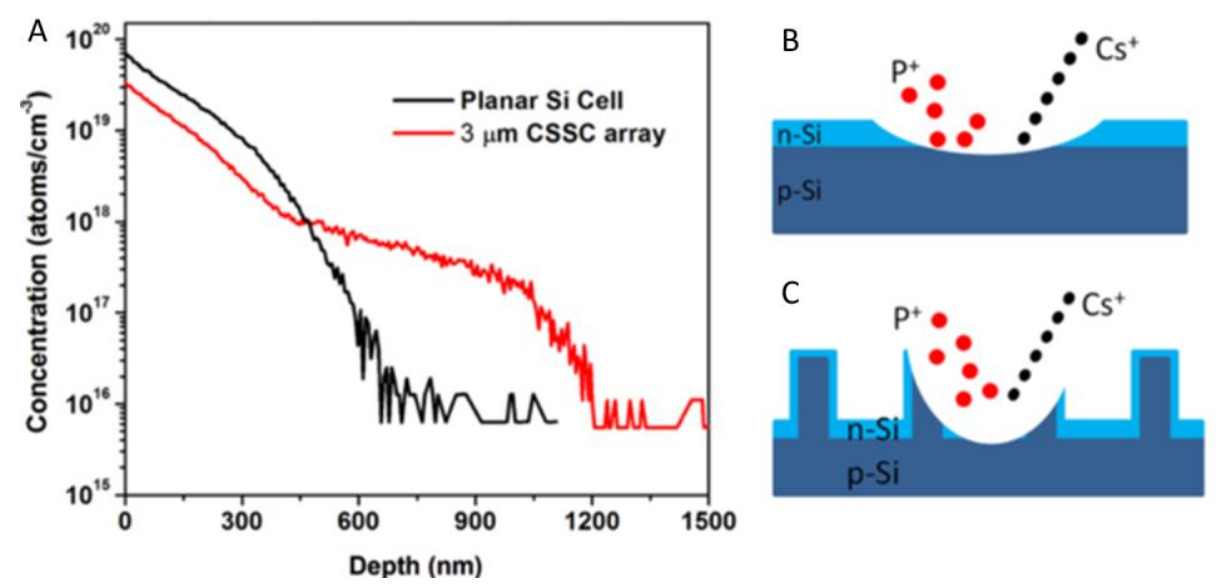

Figure 2.12: (A) SIMS measurements on both flat and structured solar cells, showing an increase in doping on structured surfaces. (B, C) Schematic illustration of SIMS measurements on flat and structured (C) silicon. Reproduced with permission. ${ }^{[22]}$ Copyright 2012, Springer.

In another example, Jin-Young et al. managed to visualize the radial doping profile in silicon micropillars using low-voltage SEM (Figure 2.13). ${ }^{[18]}$ Both nano- and micropillar arrays were fabricated using MACE, thereby obtaining a patterned micropillar array with random nanopillars in between (Figure 2.13d), which were doped using the proximity method. To observe the radial junction, cross-sectional faces were polished after filling the spacing between pillars with crystal wax (to preserve the pillars during polishing). Using decreased acceleration voltages for SEM, a contrast between the highly doped $n$-type and base $p$-type zones became visible along the vertical axis of the micropillar. The junction depth agreed with the SIMS measurements done on flat samples. Although the radial junction is visible, only the lowest $4 \mu \mathrm{m}$ of the pillar (height of $10 \mu \mathrm{m}$ ) was still present during the analysis, and it is unclear whether this is due to partial breaking of the micropillars before or after proximity doping. 


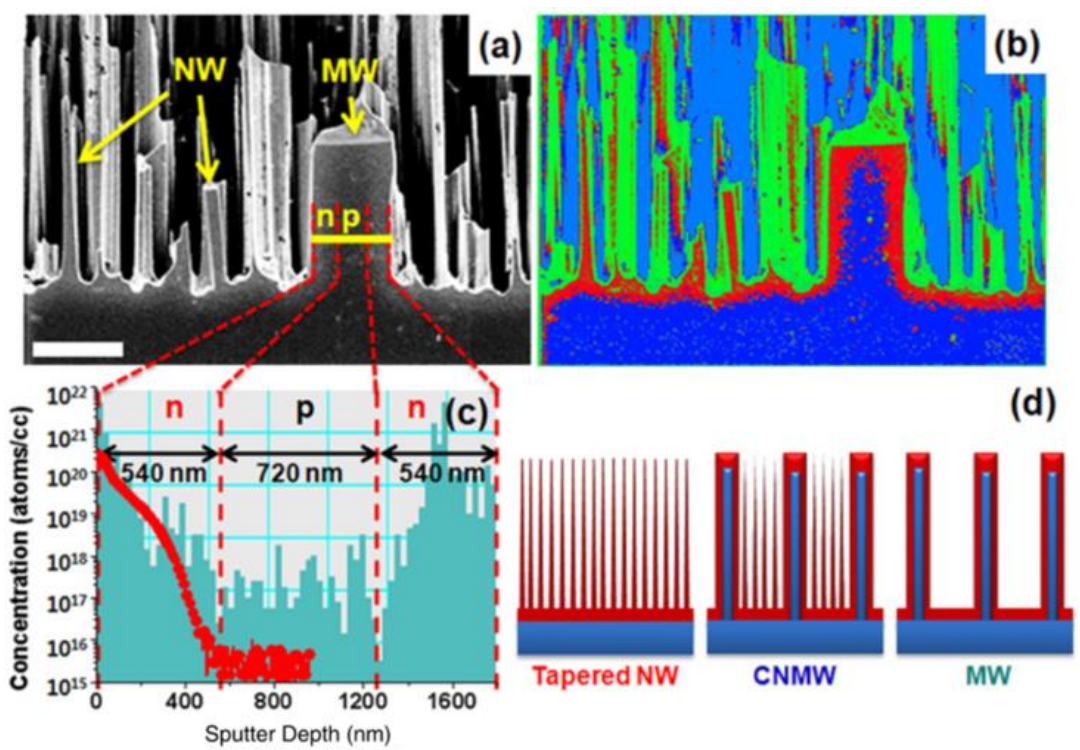

Figure 2.13: (a) Low voltage SEM cross section ( $2 \mu \mathrm{m}$ scale bar). (b) False colored image of (a), where lighter and darker parts were colored to red and blue, respectively. Green indicates unconverted areas. (c) Contrast intensity along the yellow line in (a), linked to the SIMS profile. (d) Schematic illustration of the different array configurations. Reproduced with permission. ${ }^{[18]}$ Copyright 2010, IOP Publishing.

\subsection{Optical and electrical characterization}

Besides fabrication and characterization of the pillar arrays and the $\mathrm{p} / \mathrm{n}$ junctions therein, it is equally important to analyze the optoelectronic properties of the resulting pillar arrays, in terms of the reflectivity, transmission and light absorption, and the current density-voltage (JV) characteristics, in order to quantify the improvement of the pillar arrays compared to flat surfaces.

\subsubsection{Absorption and reflection}

Pillar arrays provide a significant improvement for the generation of charge carriers, since the light is effectively trapped inside the array, an effect that can be quantified as a reduction of the reflectivity. This advantage, in combination with the decoupling of the directions of light incidence and charge transport processes, results in large improvements of the photocurrent produced by pillar arrays compared to flat surfaces. As described in section 2.2, a lot of 
research has been performed on simulations of absorption, reflectance and transmission of silicon pillar arrays, of which the common conclusion is that the pillar arrays always outperform flat surfaces. For reflectivity/transmission measurements of such highly light-scattering samples, a so-called integrating sphere is required, to be able to capture all the light. ${ }^{[110]}$ For most silicon solar cells investigated in the literature, the absorption can be directly calculated from the reflection because a thin layer $(>10 \mathrm{~nm})$ of silicon is already sufficient to prevent any transmission below a wavelength of $900 \mathrm{~nm} .^{[3]}$

The same trend as found with simulations - i.e. a large decrease of reflectance in case of the presence of nano/micron-sized pillars - has also been measured experimentally for silicon pillar arrays composed of different pillar dimensions that were made with various fabrication techniques, such as RIE, ${ }^{[111]}$ MACE $^{[112]}$ and VLS growth. ${ }^{[17]}$ For all pillar configurations, the reflectivity was below $20 \%$ (down to $<1 \%$ ) for most of the visible light wavelength range (measured for a zero degree incident angle) compared to roughly $40 \%$ for flat silicon. The angular behavior of samples with pillar arrays showed that such structured surfaces consistently outperform flat silicon surfaces, independent of the angle of light incidence or measurement angle. ${ }^{[13-115]}$ For example, DRIE nanopillars showed a $1 \%$ reflection at a $40^{\circ}$ incident angle, whereas a flat surface reflected $45 \%$ of the light. ${ }^{[14]}$ In addition, various parameters have been proven to positively affect the reflectivity, such as the diameter, ${ }^{[116]}$ tapering ${ }^{[113,117]}$ and height of the pillars. Especially the tapering of pillars, giving silicon cones with a diameter decreasing from bottom to top, significantly improves the absorption, as the flat top of a cylindrical pillar will still reflect light, an effect that is stronger at larger incident angles. ${ }^{[113]}$ Figure 2.14 shows the improvement in absorption for nanowires (pillars with a flat top, no tapering) and more specifically nanocones with a sharp tip. Although a tapered (top section of a) pillar is beneficial for light absorption, a disadvantage is that a (partially) tapered pillar has less area with a properly functioning radial junction, compared to a pillar with perfectly vertical sidewalls, which results in less collected photocurrent. 
The decrease in junction area evidently depends on the level of tapering and the junction depth.
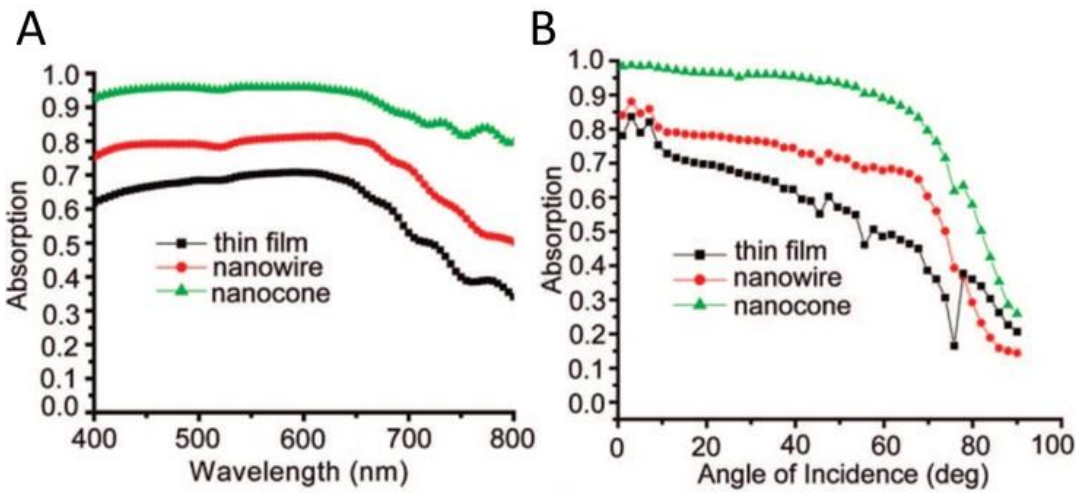

Figure 2.14: Absorption measurements on a thin film, a nanowire array and a nanocones array. (A) Absorption scanned over a wavelength range of 400-800 $\mathrm{nm}$ for all samples. (B) Angular dependency absorption measurements. Reproduced with permission. ${ }^{[13]}$ Copyright 2009, American Chemical Society

In order to obtain extremely low reflectivities, Cho et al. realized a complex light trapping pillar array, using sub-wavelength dimensions and the incorporation of extra structuring on the pillars (in the range of $30 \mathrm{~nm}$ ). ${ }^{[18]}$ Nanosized pillars were made by DRIE, and substructures on the outside of these pillars were made by a dilute polymerization and a capillary self-assembly process. This method yielded an average reflection of $<0.01 \%$, and down to even $0.0031 \%$ for wavelengths in the visible light. Doping of these nano-pillars to obtain radial $\mathrm{p} / \mathrm{n}$ junctions seems to be difficult, since the pillars tend to bend/cluster together during the solvent evaporation step.

In the case of pillars of a larger scale, with micrometer-sized diameters and heights, it is possible to further decrease the reflectivity by using anti-reflection coatings, as shown by Kelzenberg et al. ${ }^{[17]}$ To study the effect of an anti-reflective coating on wire arrays, independently of the substrate onto which the pillars were grown (by VLS), they embedded the pillars in a layer of PDMS, and transferred the PDMS-embedded arrays onto a quartz slide. The angular dependency of the absorption was measured for different anti-reflective coatings: i) an $\mathrm{SiN}_{\mathrm{x}}$ coating containing aluminum oxide nanoparticles, ii) a silver back reflector on the quartz carrier, and iii) a 
combination of both. Figure 2.15 gives an overview of the coating options, and the improvement in absorption for each coating is clearly visible. In fact, for the combined option, they achieved an absorbance of $97 \%$ for nearly the complete visible light wavelength range, and attributed the $3 \%$ loss to absorption by the PDMS. These anti-reflective coatings provide possible solutions for improvement of the absorbance of pillar arrays after fabrication of the arrays.
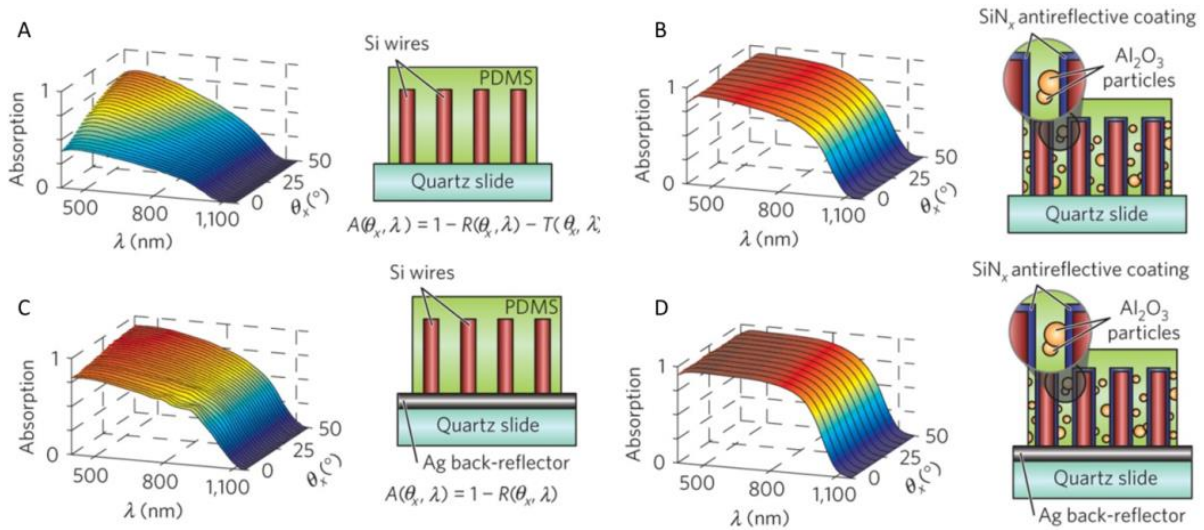

Figure 2.15: 3D absorption plots for different sets of pillar arrays, for wavelength and angular dependency. (A) Silicon pillars embedded in a PDMS layer, on top of a quartz slide. (B) Addition of an $\mathrm{SiN}_{\mathrm{x}}$ anti-reflective coating with $\mathrm{Al}_{2} \mathrm{O}_{3}$ particles. (C) Addition of a silver back reflector on top of the quartz slide, below the PDMS layer. (D) Combination of the two effects described in B and C. Reproduced with permission. ${ }^{[119]}$ Copyright 2010 , Nature Publishing Group.

\subsection{2. $J V$ measurements}

The presence of a $p / n$ junction can be verified electrically by measuring the current as a function of potential. This can also be done under illumination, as the silicon will absorb certain parts of the visible light spectrum and the $p / n$ junction will direct the resulting flow of charge carriers. By determining the short circuit current density $\left(J_{S C}\right)$ and the open circuit potential $\left(V_{O C}\right)$, the typical diode (in the dark) or solar cell (under illumination) characteristics and performance of doped pillar arrays can be determined. The improved performance of pillared surfaces over planar surfaces has been shown in numerous studies, for both solar cells ${ }^{[21,29,120]}$ and photoelectrochemical (PEC) experiments. ${ }^{[1,2,20]}$ In some cases, pillared surfaces have shown up to double 
the amount of current. ${ }^{[29]}$ Table 2.3 gives an overview of $J V$ properties of silicon cells, fabricated and doped with different techniques.

Table 2.3: Overview of $J V$ measurements on silicon solar cells, using different fabrication and doping methods on flat and structured samples.

\begin{tabular}{|c|c|c|c|c|c|}
\hline Doping method & $\begin{array}{c}\text { Fabrication } \\
\text { method }\end{array}$ & $\begin{array}{c}J_{S C} \text { Flat } \\
\left(\mathrm{mA} / \mathrm{cm}^{2}\right)\end{array}$ & $\begin{array}{l}J_{S C} \text { Pillars } \\
\left(\mathrm{mA} / \mathrm{cm}^{2}\right)\end{array}$ & $\begin{array}{c}n \text { Pillars } \\
(\%)\end{array}$ & $\begin{array}{l}\text { Junction } \\
\text { depth (nm) }\end{array}$ \\
\hline APCVD, phosphorus ${ }^{[29]}$ & DRIE & 9.6 & 20.0 & 8.7 & 300 \\
\hline SSD, phosphorus ${ }^{[86]}$ & VLS & 13.0 & $23.0^{2}$ & $9.0^{2}$ & 100 \\
\hline $\begin{array}{l}\text { Spin coating, } \\
\text { PEDOT/PSS }{ }^{[119]}\end{array}$ & MACE & 22.3 & 30.9 & 12.0 & - \\
\hline PECVD $^{1}$, boron ${ }^{[21]}$ & DRIE & 23.9 & 31.1 & 12.2 & 10 \\
\hline APCVD, phosphorus ${ }^{[120]}$ & RIE & 30.2 & 38.4 & 15.4 & 300 \\
\hline
\end{tabular}

${ }^{1}$ Doped amorphous silicon was grown in this example.

${ }^{2} \mathrm{JV}$ measurements were done a single pillar.

The $J V$ measurements give an average and global picture of the quality of the junction, but they do not give information about the homogeneity of the doped layer inside the silicon pillars or the doping profile. Due to this, it is very difficult to compare the performance of devices reported in literature, since their cell size, structural dimensions, and base doping level often vary, as well as the fabrication and doping techniques (see Table 2.3). For example, it is not possible to determine the optimal junction depth by comparing results found in literature, as too many different factors influence the $J V$ data. In addition to these dopant level and geometrical variations in pillar arrays, also antireflective coatings and passivation layers have been introduced to improve solar cells, as well as for chemical passivation of solar-to-fuel devices. ${ }^{[121,122]}$ For the fundamental understanding of a junction (i.e. recombination, resistance, junction depth) it is beneficial to compare simulation results with experiments, as shown by Christesen et al. ${ }^{[123]}$ By simulating the $J V$ data, they were able to extrapolate the limitations of their solar cell, such as the surface recombination velocity. For the understanding and comparison of different doping techniques, it is required to perform such simulations and experimental measurements for different doping settings (both on flat and nano/microstructured surfaces), to be able to make a fair estimation of optimal 
pillar and junction properties. However, to date, this is not performed frequently, nor part of the design phase of solar cells and solar-to-fuel devices.

\subsection{Conclusions}

Nano- and micropillars on silicon provide the possibility to increase the efficiency of many solar energy applications such as solar cells and solar-tofuel devices. By means of simulations of the properties of a $p / n$ junction as well as the dimensions of doped single pillars and arrays of doped pillars, the understanding of these devices is enhanced. However, experimental and modeling results are still hardly being combined.

For the fabrication of arrays of silicon nano- and micropillars, there are many options such as DRIE, VLS and MACE, all having pros and cons for making pillar arrays with certain dimensions for the pillar diameter, height and pitch. Another important step in the fabrication of solar energy devices is the formation of the required radial $\mathrm{p} / \mathrm{n}$ junction in such pillars, which can be done with a range of different techniques, ranging from bulk CVD processes, to sophisticated MLD on silicon with junction depths varying from $5 \mathrm{~nm}$ to several micrometers.

The analysis of the $p / n$ junctions is mostly restricted to planar surfaces. This is problematic since it is not yet experimentally verified whether all doping techniques are able to form a homogeneous doping profile over the full height of a pillar, especially in the case of high aspect ratio pillars or closely packed arrays. Although the presence and functionality of junctions can be proven by means of $J V$ measurements, it is still difficult to reliably select the proper design settings for solar energy devices, due to the numerous variations in literature with respect to fabrication methods, the doping profile and technique as well as pillar (diameter, height, pitch) and array (order) dimensions. Future developments will bring new insights on how to enhance the understanding, the fabrication and the performance of silicon micro/nanopillar-based solar devices. 


\subsection{References}

[1] S. W. Boettcher, E. L. Warren, M. C. Putnam, E. A. Santori, D. Turner-Evans, M. D. Kelzenberg, M. G. Walter, J. R. McKone, B. S. Brunschwig, H. A. Atwater, N. S. Lewis, J. Am. Chem. Soc., 2011, 133, 1216-1219.

[2] I. Oh, J. Kye, S. Hwang, Nano Lett., 2011, 12, 298-302.

[3] E. Garnett, P. Yang, Nano Lett., 2010, 10, 1082-1087.

[4] B. M. Kayes, H. A. Atwater, N. S. Lewis, J. Appl. Phys., 2005, 97, 1-11.

[5] J. C. Durán, G. Venier, R. Weht, Solar Cells, 1991, 31, 497-503.

[6] F. Wang, H. Yu, J. Li, S. Wong, X. W. Sun, X. Wang, H. Zheng, J. Appl. Phys., 2011, 109, $1-5$.

[7] S. M. Wong, H. Y. Yu, J. S. Li, G. Zhang, P. G. Q. Lo, D. L. Kwong, IEEE El. Device Lett., 2010, 31, 335-337.

[8] L. Hua-Min, D. Y. Lee, Y. Won Jong, Electron Devices, IEEE Transactions on, 2012, 59, 2368-2374.

[9] S.-K. Kim, R. W. Day, J. F. Cahoon, T. J. Kempa, K.-D. Song, H.-G. Park, C. M. Lieber, Nano Lett., 2012, 12, 4971-4976.

[10] L. Hu, G. Chen, Nano Lett., 2007, 7, 3249-3252.

[11] T. Xu, Y. Lambert, C. Krzeminski, B. Grandidier, D. Stiévenard, G. Lévêque, A. Akjouj, Y. Pennec, B. Djafari-Rouhani, J. Appl. Phys., 2012, 112, 033506.

[12] J. Li, H. Yu, S. M. Wong, X. Li, G. Zhang, P. G.-Q. Lo, D.-L. Kwong, Appl. Phys. Lett., 2009, 95, 243113.

[13] J. Zhu, Z. Yu, G. F. Burkhard, C.-M. Hsu, S. T. Connor, Y. Xu, Q. Wang, M. McGehee, S. Fan, Y. Cui, Nano Lett., 2008, 9, 279-282.

[14] D. Zhou, Y. Pennec, B. Djafari-Rouhani, O. Cristini-Robbe, T. Xu, Y. Lambert, Y. Deblock, M. Faucher, D. Stiévenard, J. Appl. Phys., 2014, 115, 134304.

F. Voigt, T. Stelzner, S. Christiansen, Prog. Photovolt. Res. Appl., 2012, 21, 1-13.

[16] B. C. P. Sturmberg, K. B. Dossou, L. C. Botten, A. A. Asatryan, C. G. Poulton, R. C. McPhedran, M. C. de Sterke, Appl. Phys. Lett., 2012, 101, 173902.

M. D. Kelzenberg, S. W. Boettcher, J. A. Petykiewicz, D. B. Turner-Evans, M. C. Putnam, E. L. Warren, J. M. Spurgeon, R. M. Briggs, N. S. Lewis, H. A. Atwater, Nat. Mater., 2010, 9, 239-244.

[18] J.-Y. Jung, Z. Guo, S.-W. Jee, H.-D. Um, K.-T. Park, M.S. Hyun, J.M. Yang and J.-H. Lee, Nanotechnology, 2010, 21, 445303.

[19] G.-Y. Lai, D. Kumar, Z. Pei, Nanoscale Res. Lett., 2014, 9, 1-6.

[20] Y. Hou, B. L. Abrams, P. C. K. Vesborg, M. E. Björketun, K. Herbst, L. Bech, A. M. Setti, C. D. Damsgaard, T. Pedersen, O. Hansen, J. Rossmeisl, S. Dahl, J. K. Nørskov, I. Chorkendorff, Nat. Mater., 2011, 10, 434-438.

[21] M. Gharghi, E. Fathi, B. Kante, S. Sivoththaman, X. Zhang, Nano Lett., 2012, 12, 62786282.

[22] N. Guo, J. Wei, Q. Shu, Y. Jia, S. Song, Y. Xu, H. Wang, P. Li, H. Zhu, K. Wang, D. Wu, Appl. Phys. A, 2012, 107, 911-917.

[23] C. Liu, J. Tang, H. M. Chen, B. Liu, P. Yang, Nano Lett., 2013, 13, 2989-2992.

[24] R. M. Tiggelaar, V. Verdoold, H. Eghbali, G. Desmet, J. G. E. Gardeniers, Lab Chip, 2009, 9, 456-463.

[25] H. Jansen, M. de Boer, S. Unnikrishnan, M. C. Louwerse, M. Elwenspoek, J. Micromech. Microeng., 2009, 19, 033001.

[26] I. W. Rangelow, J. Vac. Sci. Technol. A, 2003, 21, 1550-1562.

[27] M. L. Hoarfrost, K. Takei, V. Ho, A. Heitsch, P. Trefonas, A. Javey, R. A. Segalman, J. Phys. Chem. Lett., 2013, 4, 3741-3746.

[28] J. op de Beeck, W. de Malsche, D. S. Tezcan, P. de Moor, G. Desmet, J. Chromatogr. A, 2012, 1239, 35-48.

[29] H. P. Yoon, Y. A. Yuwen, C. E. Kendrick, G. D. Barber, N. J. Podraza, J. M. Redwing, T. E. Mallouk, C. R. Wronski, T. S. Mayer, Appl. Phys. Lett., 2010, 96, 213503.

[30] M. D. Henry, S. Walavalkar, A. Homyk, A. Scherer, Nanotechnology, 2009, 20, 255305.

[31] R. Ji, W. Lee, R. Scholz, U. Gösele, K. Nielsch, Adv. Mater., 2006, 18, 2593-2596.

[32] Y. Qu, L. Liao, R. Cheng, Y. Wang, Y.-C. Lin, Y. Huang, X. Duan, Nano Lett., 2010, 10, 1941-1949. 
[33] C. L. Cheung, R. J. Nikolić, C. E. Reinhardt, T. F. Wang, Nanotechnology, 2006, 17, 1339.

J. Liu, M. Ashmkhan, B. Wang, F. Yi, Appl. Surf. Sci., 2012, 258, 8825-8830.

K. J. Morton, G. Nieberg, S. Bai, S. Y. Chou, Nanotechnology, 2008, 19, 345301.

M. D. Austin, H. Ge, W. Wu, M. Li, Z. Yu, D. Wasserman, S. A. Lyon, S. Y. Chou, Appl. Phys. Lett., 2004, 84, 5299-5301.

[37] M. S. Schmidt, J. Hübner, A. Boisen, Adv. Mater., 2012, 24, OP11-OP18.

[38] H. Jansen, M. de Boer, R. Legtenberg, M. Elwenspoek, J. Micromech. Microeng., 1995, $5,115$.

R. Schuster, V. Kirchner, P. Allongue, G. Ertl, Science, 2000, 289, 98-101.

G. Barillaro, A. Nannini, F. Pieri, J. Electrochem. Soc., 2002, 149, C180-C185.

[43] M. Bassu, S. Surdo, L. M. Strambini, G. Barillaro, Adv. Funct. Mater., 2012, 22, $1222-$ 1228.

[44] K. Q. Peng, Y. J. Yan, S. P. Gao, J. Zhu, Adv. Mater., 2002, 14, 1164-1167.

[45] Z. Huang, N. Geyer, P. Werner, J. de Boor, U. Gösele, Adv. Mater., 2011, 23, 285-308.

[46] N. Geyer, B. Fuhrmann, Z. P. Huang, J. de Boor, H. S. Leipner, P. Werner, J. Phys. Chem. C, 2012, 116, 13446-13451.

[48] Z. P. Huang, X. X. Zhang, M. Reiche, L. F. Liu, W. Lee, T. Shimizu, S. Senz, U. Gosele, Nano Lett., 2008, 8, 3046-3051.

[49] N. Kwon, N. Kim, S. Sung, B. Kang, I. Chung, J. Vac. Sci. Technol. B, 2012, 30, 041810.

K. Peng, A. Lu, R. Zhang, S.-T. Lee, Adv. Funct. Mater., 2008, 18, 3026-3035.

[53] S. Barth, F. Hernandez-Ramirez, J. D. Holmes, A. Romano-Rodriguez, Prog. Mater. R. S. Wagner, W. C. Ellis, Appl. Phys. Lett., 1964, 4, 89-90.

[54] T. Mårtensson, M. Borgström, W. Seifert, B. J. Ohlsson, L. Samuelson, Nanotechnology, 2003, 14, 1255.

[55] J. B. Hannon, S. Kodambaka, F. M. Ross, R. M. Tromp, Nature, 2006, 440, 69-71.

[56] T. Shimizu, T. Xie, J. Nishikawa, S. Shingubara, S. Senz, U. Gösele, Adv. Mater., 2007, 19, 917-920.

B. M. Kayes, M. A. Filler, M. C. Putnam, M. D. Kelzenberg, N. S. Lewis, H. A. Atwater, Appl. Phys. Lett., 2007, 91, 1-3.

[58] K. E. Plass, M. A. Filler, J. M. Spurgeon, B. M. Kayes, S. Maldonado, B. S. Brunschwig, H. A. Atwater, N. S. Lewis, Adv. Mater., 2009, 21, 325-328.

[59] J. Op de Beeck, W. De Malsche, P. De Moor, G. Desmet, J. Sep. Sci., 2012, 35, 18771883.

[60] K. Peng, Y. Yan, S. Gao, J. Zhu, Adv. Funct. Mater., 2003, 13, 127-132.

[61] B. S. Meyerson, Proceedings of the IEEE, 1992, 80, 1592-1608.

[62] P. A. Stolk, H.-J. Gossmann, D. J. Eaglesham, D. C. Jacobson, C. S. Rafferty, G. H. Gilmer, M. Jaraíz, J. M. Poate, H. S. Luftman, T. E. Haynes, J. Appl. Phys., 1997, 81, 6031-6050.

[63] T. Michel, J. Le Perchec, A. Lanterne, R. Monna, F. Torregrosa, L. Roux, M. Commandré, Sol. Energy Mater. Sol. Cells, 2015, 133, 194-200.

[64] Y. H. Lee, M. M. Chen, J. Vac. Sci. Technol. B, 1986, 4, 468-475.

[65] Y. H. Lee, M. M. Chen, A. A. Bright, Appl. Phys. Lett., 1985, 46, 260-262.

[66] P. Rothhardt, C. Demberger, A. Wolf, D. Biro, Energy Procedia, 2013, 38, 305-311.

[67] C. Gong, K. van Nieuwenhuysen, N. E. Posthuma, E. van Kerschaver, J. Poortmans, Sol. Energy Mater. Sol. Cells, 2011, 95, 11-13.

[68] J. Engelhardt, A. Frey, L. Mahlstaedt, S. Gloger, G. Hahn, B. Terheiden, Energy Procedia, 2014, 55, 235-240.

[69] L. Yu, S. Misra, J. Wang, S. Qian, M. Foldyna, J. Xu, Y. Shi, E. Johnson, P. R. i. Cabarrocas, Sci. Rep., 2014, 4.

[70] K. E. Moselund, H. Ghoneim, H. Schmid, M. T. Björk, E. Lörtscher, S. Karg, G. Signorello, D. Webb, M. Tschudy, R. Beyeler, H. Riel, Nanotechnology, 2010, 21, 435202. 
[71] L. Yu, B. O’Donnell, M. Foldyna, P. R. i Cabarrocas, Nanotechnology, 2012, 23, 194011.

[72] U. Kroll, C. Bucher, S. Benagli, I. Schönbächler, J. Meier, A. Shah, J. Ballutaud, A. Howling, C. Hollenstein, A. Büchel, M. Poppeller, Thin Solid Films, 2004, 451-452, 525530.

[73] R. E. Schropp, ECS Trans., 2009, 25, 3-14.

[74] J. Cifre, J. Bertomeu, J. Puigdollers, M. C. Polo, J. Andreu, A. Lloret, Appl. Phys. A, 1994, 59, 645-651.

[75] Q. Wang, S. Ward, L. Gedvilas, B. Keyes, E. Sanchez, S. Wang, Appl. Phys. Lett., 2004, 84, 338-340.

[76] H. Taro, N. Yuki, K. Koichi, O. Keisuke, M. Hideki, Jpn. J. Appl. Phys., 2012, 51, 061301.

[77] G. Dong, F. Liu, J. Liu, H. Zhang, M. Zhu, Nanoscale Res. Lett., 2013, 8, 544.

[78] M. Kupich, P. Kumar, B. Schröder, Thin Solid Films, 2003, 430, 236-239.

[79] F. Sarubbi, L. K. Nanver, T. L. Scholtes, ECS Trans., 2006, 3, 35-44.

[80] A. D. McConnell, S. Uma, K. E. Goodson, J. Microelectromech. Syst., 2001, 10, 360369.

[81] B. S. Meyerson, W. Olbricht, J. Electrochem. Soc., 1984, 131, 2361-2365.

[82] R. R. King, E. W. Thomas, W. B. Carter, A. Rohatgi, IEEE Phot. Spec. Conf., New York, 1991.

[83] R. Hull, Properties of Crystalline Silicon, The Institution of Electrical Engineers, United Kingdom, 1999.

[84] R. M. Tiggelaar, P. van Male, J. W. Berenschot, J. G. E. Gardeniers, R. E. Oosterbroek, M. H. J. M. de Croon, J. C. Schouten, A. van den Berg, M. C. Elwenspoek, Sens. Actuators, A, 2005, 119, 196-205.

[85] J. R. Flemish, R. E. Tressler, J. Ruzyllo, J. Electrochem. Soc., 1991, 138, 233-238.

[86] M. D. Kelzenberg, D. B. Turner-Evans, M. C. Putnam, S. W. Boettcher, R. M. Briggs, J. Y. Baek, N. S. Lewis, H. A. Atwater, Energy Environ. Sci., 2011, 4, 866-871.

[87] J. C. Ho, R. Yerushalmi, Z. A. Jacobson, Z. Fan, R. L. Alley, A. Javey, Nat. Mater., 2008, 7, 62-67.

[88] W. P. Voorthuijzen, M. D. Yilmaz, W. J. M. Naber, J. Huskens, W. G. van der Wiel, Adv. Mater., 2011, 23, 1346-1350.

[89] K. W. Ang, J. Barnett, W. Y. Loh, J. Huang, B. G. Min, P. Y. Hung, I. Ok, J. H. Yum, G. Bersuker, M. Rodgers, V. Kaushik, S. Gausepohl, C. Hobbs, P. D. Kirsch, R. Jammy, Int. El. Devices Meet., 2011.

[90] S. R. McKibbin, C. M. Polley, G. Scappucci, J. G. Keizer, M. Y. Simmons, Appl. Phys. Lett., 2014, 104, 123502.

[91] R. C. Longo, K. Cho, W. G. Schmidt, Y. J. Chabal, P. Thissen, Adv. Funct. Mater., 2013, 23, 3471-3477.

[92] J. C. Ho, R. Yerushalmi, G. Smith, P. Majhi, J. Bennett, J. Halim, V. N. Faifer, A. Javey, Nano Lett., 2009, 9, 725-730.

[93] C. Gopalan, P. S. Chakraborty, J. Yang, K. Taehoon, W. Zhiyuan, M. R. McCartney, S. M. Goodnick, M. N. Kozicki, T. J. Thornton, IEEE Trans. El. Devices, 2003, 50, 12771283.

[94] S.-H. Baek, B.-Y. Noh, I.-K. Park, J. H. Kim, Nanoscale Res. Lett., 2012, 7, 29.

[95] Z.-T. Zhu, E. Menard, K. Hurley, R. G. Nuzzo, J. A. Rogers, Appl. Phys. Lett., 2005, 86, 133507.

[96] J. S. Sadhu, H. Tian, T. Spila, J. Kim, B. Azeredo, P. Ferreira, S. Sinha, Nanotechnology, 2014, 25, 375701.

[97] W. Zagozdzon-Wosik, P. B. Grabiec, G. Lux, J. Appl. Phys., 1994, 75, 337-344.

[98] S. Ingole, P. Aella, P. Manandhar, S. B. Chikkannanavar, E. A. Akhadov, D. J. Smith, S. T. Picraux, J. Appl. Phys., 2008, 103, 104302-104308.

[99] O. Hazut, A. Agarwala, I. Amit, T. Subramani, S. Zaidiner, Y. Rosenwaks, R. Yerushalmi, ACS Nano, 2012, 6, 10311-10318.

[100] O. Hazut, B.-C. Huang, A. Pantzer, I. Amit, Y. Rosenwaks, A. Kohn, C.-S. Chang, Y.-P. Chiu, R. Yerushalmi, ACS Nano, 2014, 8, 8357-8362.

[101] W. L. Ng, M. A. Lourenco, R. M. Gwilliam, S. Ledain, G. Shao, K. P. Homewood, Nature, 2001, 410, 192-194. 
[102] S. C. Jain, W. Schoenmaker, R. Lindsay, P. A. Stolk, S. Decoutere, M. Willander, H. E. Maes, J. Appl. Phys., 2002, 91, 8919-8941.

[103] M. Uematsu, J. Appl. Phys., 1997, 82, 2228-2246.

[104] Y. M. Haddara, B. T. Folmer, M. E. Law, T. Buyuklimanli, Appl. Phys. Lett., 2000, 77, 1976-1978.

[105] R. G. Mazur, D. H. Dickey, J. Electrochem. Soc., 1966, 113, 255-259.

[106] R. A. Puglisi, C. Garozzo, C. Bongiorno, S. Di Franco, M. Italia, G. Mannino, S. Scalese, A. La Magna, Sol. Energy Mater. Sol. Cells, 2015, 132, 118-122.

[107] G. Bose, IC Fabrication Technology, McGraw Hill, New Delhi, 2014.

[108] W. C. Roman, L. R. Wilson, US Patent 3830665, 1974.

[109] B. Hoex, J. Schmidt, R. Bock, P. P. Altermatt, M. C. M. van de Sanden, W. M. M. Kessels, Appl. Phys. Lett., 2007, 91, 1-3.

[110] J. C. de Mello, H. F. Wittmann, R. H. Friend, Adv. Mater., 1997, 9, 230-232.

[111] Z. Yu, H. Gao, W. Wu, H. Ge, S. Y. Chou, J. Vac. Sci. Technol. B, 2003, 21, 2874-2877.

[112] P. Pushpa Raj, E. David, A. A. Arturo, J. Phys. D: Appl. Phys., 2013, 46, 235104.

[113] J. Zhu, Z. Yu, G. F. Burkhard, C.-M. Hsu, S. T. Connor, Y. Xu, Q. Wang, M. McGehee, S. Fan, Y. Cui, Nano Lett., 2009, 9, 279-282.

[114] Y. Hung, Jr., S.-L. Lee, L. A. Coldren, Opt. Express, 2010, 18, 6841-6852.

[115] K. Sun, S. Shen, Y. Liang, P. E. Burrows, S. S. Mao, D. Wang, Chem. Rev., 2014, 114, 8662-8719.

[116] F. J. Bezares, J. P. Long, O. J. Glembocki, J. Guo, R. W. Rendell, R. Kasica, L. Shirey, J. C. Owrutsky, J. D. Caldwell, Opt. Express, 2013, 21, 27587-27601.

[117] H. Xu, N. Lu, D. Qi, J. Hao, L. Gao, B. Zhang, L. Chi, Small, 2008, 4, 1972-1975.

[118] S. J. Cho, T. An, G. Lim, Chem. Commun. (Cambridge, U. K.), 2014, 50, 15710-15713.

[119] J. Zhang, T. Song, X. Shen, X. Yu, S.-T. Lee, B. Sun, ACS Nano, 2014, 8, 1136911376

[120] J. Liu, X. Zhang, G. Dong, Y. Liao, B. Wang, T. Zhang, F. Yi, Appl. Phys. A, 2014, 4, $1175-1179$.

[121] M. C. Putnam, S. W. Boettcher, M. D. Kelzenberg, D. B. Turner-Evans, J. M. Spurgeon, E. L. Warren, R. M. Briggs, N. S. Lewis, H. A. Atwater, Energy Environ. Sci., 2010, 3, 1037-1041.

[122] D. R. Kim, C. H. Lee, P. M. Rao, I. S. Cho, X. Zheng, Nano Lett., 2011, 11, 2704-2708.

[123] J. D. Christesen, X. Zhang, C. W. Pinion, T. A. Celano, C. J. Flynn, J. F. Cahoon, Nano Lett., 2012, 12, 6024-6029. 


\section{Chapter 3 \\ Controlled Doping Methods for Radial $\mathbf{p} / \mathbf{n}$ Junctions in Silicon Micropillars}

$\mathrm{P} / \mathrm{n}$ and $\mathrm{n} / \mathrm{p}$ junctions with depths of $200 \mathrm{~nm}$ to several microns have been created in flat silicon substrates as well as on $3 D$ microstructures by means of a variety of methods, including solid source dotation (SSD), low-pressure chemical vapor deposition (LPCVD), atmospheric-pressure chemical vapor deposition (APCVD) and plasma-enhanced chemical vapor deposition (PECVD). Radial junctions in silicon micropillars were inspected by optical and scanning electron microscopies, using a $\mathrm{CrO}_{3}$ based staining solution, which enabled visualization of the junction depth. When applying identical doping parameters to flat substrates, ball grooving, followed by staining and optical microscopy, yielded similar junction depth values as HR-SEM imaging on stained cross-sections and SIMS depth profilometry. For the investigated $3 D$ microstructures, doping based on SSD and LPCVD gave uniform and conformal junctions. Junctions made with SSD-boron doping and CVD-phosphorus doping could be accurately predicted with a model based on Fick's diffusion law. 3D microstructured silicon pillar arrays showed an increased efficiency for sunlight capturing. The functionality of micropillar arrays with radial junctions was evidenced by improved short-circuit current densities and photovoltaic efficiencies compared to flat surfaces, for both $n$ - and p-type wafers (pillar arrays efficiencies of $9.4 \%$ and $11 \%$, respectively).

This chapter has been published as: R. Elbersen, R.M. Tiggelaar, A. Milbrat, G. Mul, J.G.E. Gardeniers, J. Huskens, Adv. Energy Mater., 2015, 5, 1401745 


\subsection{Introduction}

In the field of solar energy technology, (sub)micron p/n junctions in crystalline silicon are used for achieving charge separation at the surface of a solar cell. ${ }^{[1,2]}$ To increase the efficiency of light capturing of solar cells, research has focused on nano/micro structures, e.g. wires, with shallow junctions. ${ }^{[3,4]}$ Such structures have advantages over thin film and bulk silicon surfaces, such as higher surface areas and improved light-trapping capabilities. ${ }^{[5-8]}$ Although both nano- and microstructures can be utilized, nanostructures put lower demands on the silicon quality due to shorter minority carrier diffusion lengths, ${ }^{[9]}$ whereas micron-sized features can be created with more commonly used fabrication methods.

Silicon micropillars, with diameters of a few micrometers and heights of tens of micrometers containing radial $\mathrm{p} / \mathrm{n}$ junctions, have also received increased attention, not only for solar cells, but also for solar-to-fuel applications, as they can be functionalized in a controlled manner with various catalysts. ${ }^{[10-13]}$ Using radial junctions, increased efficiencies over flat surfaces can be obtained, due to greatly enhanced carrier collection in nano/micropillars. ${ }^{[7,13]}$ In addition, the radial junction attributes to a lower surface recombination rate for the overall device. ${ }^{[14,15]}$

Here, we investigate different doping methods applied to silicon micropillars, in order to obtain optimal geometry control of the pillars and $p / n$ junctions therein, as well as ease of utilization for further applications. Silicon-based $p / n$ junctions can be realized by various doping methods. Standard doping techniques reported in literature include ion implantation, ${ }^{[16]}$ solid source dotation (SSD), ${ }^{[17]}$ monolayer doping, ${ }^{[18]}$ spin-on dopant, ${ }^{[19]}$ as well as regular (CVD), low-pressure (LPCVD), plasma-enhanced (PECVD) and atmospheric-pressure (APCVD) chemical vapor deposition. ${ }^{[20]}$ Not all techniques are suited for 3D structures, for example, ion implantation is limited to flat surfaces because of its directionality, while PECVD, in contrast to 
LPCVD, does not give conformal step coverages, except for highly tuned process conditions. ${ }^{[21]}$ Furthermore, high-density pillar arrays with small pillar spacing and/or large aspect ratios can suffer from non-uniform doping of the $3 \mathrm{D}$ structures, in particular across the height of the structures. ${ }^{[2,23]}$ Thorough investigations of whether common doping techniques lead to a controlled junction depth in doped micro- and/or nanopillars have not been reported. Here, we present different doping methods for both n-type (phosphorus, P) and p-type (boron, B) doping of 3D crystalline silicon microstructures. We used SSD in the case of boron doping, and several CVD techniques (LPCVD, $A P C V D$ and PECVD) for phosphorous doping. For comparison, junction depth analysis after doping with these techniques is done also on flat surfaces, using ball grooving and chemical staining. Junctions in ridges and micropillars were analyzed by cross sectional imaging using high resolution scanning electron microscopy (HR-SEM). Secondary ion mass spectrometry (SIMS) was applied to flat samples to verify junction depths and surface concentrations. JV measurements were performed under the standardized AM (air mass) 1.5G (global) illumination. The experimental results were compared with finite element calculations of the dopant diffusion processes in flat and pillar structures. This combination of experimental analysis and numerical simulations forms a route to determine the optimal settings for structured solar cells and solar-to-fuel devices.

\subsection{Materials and methods}

\subsubsection{Fabrication of microstructure arrays}

On p- and n-type silicon substrates ((100)-oriented, resistivity 5-10 $\Omega \mathrm{cm}$ (p-type) and 1-10 $\Omega \mathrm{cm}$ (n-type), $100 \mathrm{~mm}$ diameter, thickness $525 \mu \mathrm{m}$ (p-type) and $375 \mu \mathrm{m}$ (n-type), single side polished, Okmetic Finland), arrays of silicon microstructures were fabricated. Prior to processing, the substrates were cleaned by immersion in $100 \%$ nitric acid $\left(\mathrm{HNO}_{3}\right)$ (2x $5 \mathrm{~min}$ ), and in fuming $69 \%$ nitric acid (15 min), which was followed by quick dump rinsing in de-mineralized (DI) water, immersion in $1 \%$ aqueous hydrofluoric acid (HF) to 
remove the native oxide prior to silicon nitride deposition and another quick dump rinsing cycle. After spin drying $(6000 \mathrm{rpm})$ of the wafers, $100 \mathrm{~nm}$ thick silicon-rich silicon nitride (SiRN) was deposited using low-pressure chemical vapor deposition (LPCVD), to prevent doping the backside of the wafer. With reactive ion etching (RIE; Adixen AMS100DE; octafluorocyclobutane $\left(\mathrm{C}_{4} \mathrm{~F}_{8}\right)$ and methane $\left.\left(\mathrm{CH}_{4}\right)\right)$ the SiRN layer on the front side of the wafer was removed, followed by an oxygen plasma treatment and piranha (mixture of sulfuric acid and 30\% aqueous hydrogen peroxide, 3:1 (v/v), $20 \mathrm{~min}$ ) cleaning to remove any contamination. For use as a hard mask during etching, a layer of $2 \mu \mathrm{m}$ silicon dioxide $\left(\mathrm{SiO}_{2}\right)$ was grown using wet oxidation $\left(1150{ }^{\circ} \mathrm{C}\right)$. By means of standard UV-lithography (on wafer-scale) for each $2 \times 2 \mathrm{~cm}^{2}$ sample, a centered $0.5 \times 0.5 \mathrm{~cm}^{2}$ area with an array of micropillars (diameter $4 \mu \mathrm{m}$, spacing $2 \mu \mathrm{m}$, hexagonally stacked with a packing density of $35 \%$ ) or ridges (width $100 \mu \mathrm{m}$, spacing $100 \mu \mathrm{m}$, height $30 \mu \mathrm{m}$ ) was defined in photoresist (Olin 907-17), and post-baked for $10 \mathrm{~min}$ at $120{ }^{\circ} \mathrm{C}$ after development. The photoresist pattern was transferred into the $\mathrm{SiO}_{2}$ layer by means of RIE (Adixen AMS100DE; $\mathrm{C}_{4} \mathrm{~F}_{8}, \mathrm{CH}_{4}$ ). The photoresist, in combination with the $\mathrm{SiO}_{2}$, acted as a mask layer during deep reactive ion etching (DRIE; Adixen AMS100SE) of silicon using the Bosch process, i.e. a cyclic process employing sulfur hexafluoride $\left(\mathrm{SF}_{6}\right)$ for etching silicon and $\mathrm{C}_{4} \mathrm{~F}_{8}$ to create a passivation layer on the sidewalls. The height of the pillars was determined by the etch duration, and was set to $20 \mathrm{~min}$, resulting in pillar heights of approximately $60 \mu \mathrm{m}$. After etching, the photoresist mask was stripped with oxygen plasma, followed by piranha cleaning (20 min). Subsequently, the $\mathrm{SiO}_{2}$ layer was removed with $50 \%$ aqueous $\mathrm{HF}$. The substrate was cleaned from the remaining fluorocarbons in the DRIE process by oxidizing the surface at $800{ }^{\circ} \mathrm{C}$ (30 min), immersion in $1 \%$ aqueous HF (10 min), rinsing in DI water and drying. 


\subsubsection{Doping methods}

Doping of the arrays of silicon micropillars was done using various techniques, i.e. SSD, LPCVD, APCVD and PECVD. In all cases, a dopant-containing oxide layer was formed on the silicon surface, which was followed by a thermal drive-in step to transfer the dopant into the silicon. In this work, the drive-in temperature ranged from $900-1050^{\circ} \mathrm{C}$ and the drive-in time was in the range of $15-120 \mathrm{~min}$. Prior to processing, all wafers were immersed in $1 \%$ aqueous hydrofluoric acid (10 min), rinsing in DI water and drying, to expose a $\mathrm{H}$-terminated silicon surface. To remove the dopant oxide layer after the doping process, wafers were immersed in buffered hydrogen fluoride (1:7, BHF, $10 \mathrm{~min}$ ), oxidized at $800{ }^{\circ} \mathrm{C}$ for $15 \mathrm{~min}$ and etched in $1 \% \mathrm{HF}$ (15 $\mathrm{min}$ ), rinsed and dried. Below for each doping method details are given on the formation of the dopant containing oxide layer.

\section{Solid source dotation}

In the case of solid source dotation (SSD) of boron, n-type silicon wafers were placed in-between boron nitride wafers and a thin layer $(\sim 200 \mathrm{~nm})$ of boron oxide $\left(\mathrm{B}_{2} \mathrm{O}_{5}\right)$ was formed on the surface of the silicon wafers under a continuous oxygen flow $(6000 \mathrm{sccm})$ at $800{ }^{\circ} \mathrm{C}$.

\section{Chemical vapor deposition}

For chemical vapor deposition (CVD), three different processes were used; an atmospheric-pressure CVD (APCVD), a low-pressure CVD (LPCVD), and a plasma-enhanced CVD (PECVD). For the APCVD of phosphorus on p-type $\mathrm{Si}$, gas phase deposition was used to create a dopant oxide layer: at $950{ }^{\circ} \mathrm{C}$, a mixture of $4500 \mathrm{sccm}$ phosphine $\left(\mathrm{PH}_{3}\right)$ and $1200 \mathrm{sccm} \mathrm{O}$ was flushed through the furnace for $30 \mathrm{~min}$. In the LPCVD process, wafers were loaded in a boat filled with dummy wafers, for optimal growth conditions on wafers. For a deposition time of $30 \mathrm{~min}$ at $650^{\circ} \mathrm{C}$, a gas flow of $330 \mathrm{sccm} \mathrm{PH}_{3}$ and $150 \mathrm{sccm}$ $\mathrm{O}_{2}$ was used to grow the phosphorus oxide (at a pressure of 350 mTorr). PECVD was utilized to deposit a phosphorus glass layer on flat $p$-Si wafers. At 
$300{ }^{\circ} \mathrm{C}$ and $1050 \mathrm{mTorr}$, a gas flow of $\mathrm{PH}_{3}(100 \mathrm{sccm})$ was flushed through the chamber for $15 \mathrm{~min}$, in combination with $200 \mathrm{sccm} \mathrm{N}_{2} \mathrm{O}$ and $700 \mathrm{sccm} \mathrm{N}_{2}$. A DC voltage of $50 \mathrm{~W}$ was used.

\subsubsection{Analysis methods}

\section{Ball grooving and staining}

To analyze the $\mathrm{p} / \mathrm{n}$ junctions on flat surfaces, a stainless steel ball $(60 \mathrm{~mm}$ diameter) was used to expose the junction, by grooving the surface. ${ }^{[24]}$ To improve the grooving rate, diamond paste was applied to the ball. Typically only a few seconds were required to grind sufficiently deep (i.e. through the junction) to a depth of 2-3 $\mu \mathrm{m}$. After grooving, the samples were cleaned with ethanol.

Revealing the depth of a p/n junction was done using an etching solution for delineation along the $\mathrm{p} / \mathrm{n}$ junction region, following the procedure as described in a patent by Roman and Wilson. ${ }^{[22]}$ Chromium trioxide $\left(\mathrm{CrO}_{3}\right)$ was mixed with DI water in a ratio of 1 to $3(\mathrm{w} / \mathrm{w})$, subsequently a $50 \%$ aqueous $\mathrm{HF}$ solution (10 v\% of the starting solution) was added to the $\mathrm{CrO}_{3}$ solution. Samples were placed in the resulting solution for $25 \mathrm{~s}$, and subsequently rinsed with DI water and dried with a stream of nitrogen. After this staining reaction, a contrast difference is visible under a normal light microscope, in which the p-type silicon area becomes darker than the n-type silicon. Figure 3.1 shows schematic cross-sectional and top views of a stained groove. The same is possible for $n$-type doping in a p-type wafer. Although the contrast is less pronounced in this case, the contrast circle is still clearly visible. 

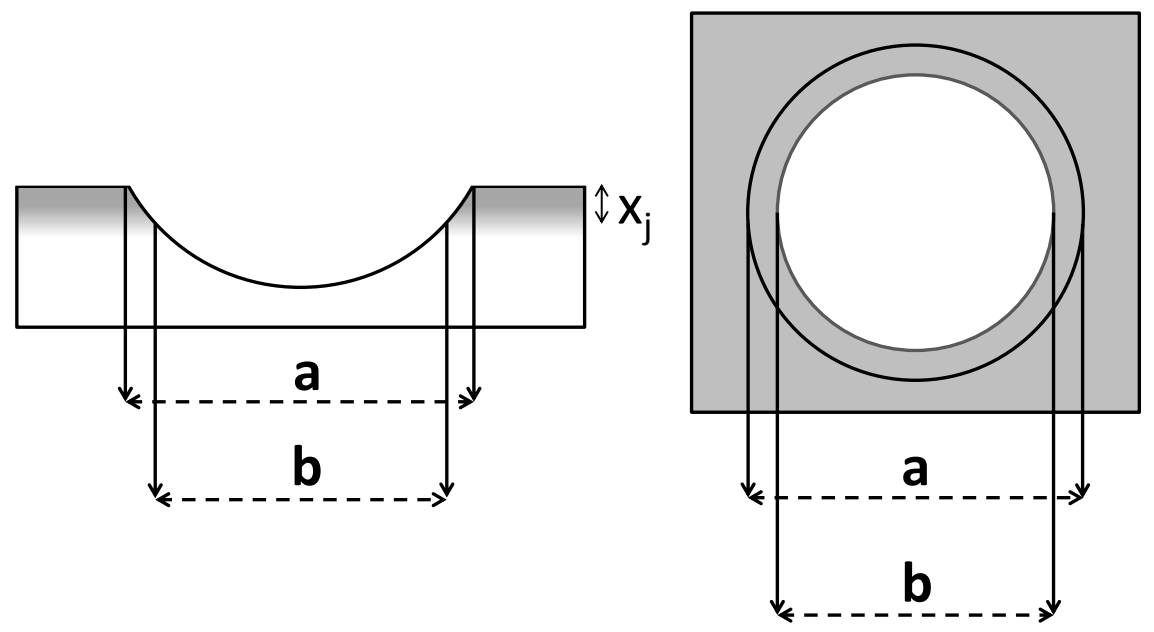

Figure 3.1: Schematic cross section (left) and top view (right) of a stained groove on a flat silicon surface. The dark-gray area indicates the boron-doped silicon layer, whereas the white part is the bulk n-type silicon.

To calculate the junction depth $\left(x_{j}\right)$ after staining of the groove, the diameters $(a, b)$ of the two formed circles were determined. Then $x_{j}(\mu \mathrm{m})$ was calculated as:

$$
x_{j}=\frac{a^{2}-b^{2}}{8 * R}
$$

where $a$ is the outer diameter of the circle $(\mu \mathrm{m}), b$ the inner diameter $(\mu \mathrm{m})$ and $R$ the radius of the stainless steel ball $(\mu \mathrm{m})$. A light microscope (Olympus BHMJL, 5x magnification, Analysis ${ }^{\circledR}$ software) was used to measure $a$ and $b$.

\section{High-resolution scanning electron microscopy}

To analyze the junctions, HR-SEM images of cross-sections of doped ridges and micropillars were taken on an Analysis Zeiss-Merlin HR-SEM system with an InLens detector. For ridges, samples were broken with a diamond pen perpendicular to the length of the ridges. Focused ion beam (FIB) and reactive ion etching were used to open up and image the micropillars. FIB structures and images were made with a Nova 600 DualBeam - SEM/FIB setup. $\mathrm{A} \mathrm{Ga}^{+}$liquid metal ion source was used to mill away enough of a pillar to be 
able to accurately determine the junction location, with a beam current of $0.92 \mathrm{nA}$ and $10 \mathrm{kV}$ extraction voltage.

\section{Secondary ion mass spectrometry measurements}

Secondary ion mass spectrometry (SIMS) depth profiles of doped flat samples were recorded using a Cameca ims6f using $7.5 \mathrm{keV} \mathrm{O}_{2}^{+}$primary ions in positive mode. Secondary ions $\left({ }^{31} \mathrm{P}^{+}\right.$or $\left.{ }^{11} \mathrm{~B}^{+}\right)$and ${ }^{28} \mathrm{Si}^{28} \mathrm{Si}^{+}$as a reference were detected. Quantification and depth calibration was based on reference implants. Depth scale calibration was based on final crater depth measurements using optical profilometry. For each dopant setting, only one measurement was performed.

\section{Electrical characterization}

To investigate the electrical properties of the formed junctions, front and backside contacts were made by sputtering $1 \mu \mathrm{m}$ aluminum/silicon alloy (99\% aluminum, 1\% silicon). Samples were placed perpendicular to a $300 \mathrm{~W}$ xenon arc light source, which was calibrated to match the intensity of 1 sun (AM 1.5). In case of $\mathrm{p} / \mathrm{n}$ junctions created on low-doped $n$-type silicon $\left(\sim 10^{15}\right.$ atoms $\left./ \mathrm{cm}^{3}\right)$, the backside of samples was doped with phosphorus (similar to the procedure for junction formation) to create $\mathrm{n}^{+}$silicon. This was necessary to ensure an Ohmic contact between the aluminum/silicon alloy and n-type silicon. JV measurements were recorded on a VersaSTAT 4 potentiostat. For each dopant setting, at least 5 different samples were measured.

Finite element simulations of boron and phosphorus doping

Junction depths and doping concentrations of $p / n$ junctions created with SSD, LPCVD and PECVD were simulated in COMSOL Multiphysics (version 4.4) using the finite element method (FEM). All simulations were done with a timedependent transport of diluted species on rod-like structures of various dimensions (similar to the realized pillar arrays), using a free tetrahedral mesh, with a maximum mesh size of $0.5 \mu \mathrm{m}$ and a minimum of $1 \mathrm{~nm}$. 
The boron (or phosphorus) oxide source was simulated as an infinite source of dopant atoms, with a fixed surface concentration $\left(\sim 10^{22}\right.$ atoms $\left./ \mathrm{cm}^{3}\right)$. Three drive-in temperatures were simulated in time, i.e. 900, 1000 and $1050{ }^{\circ} \mathrm{C}$. Although heating up and cooling down of the furnace was also included in the simulation, the mentioned drive-in times always correspond to the duration of the drive-in temperature step after stabilization to its desired value.

\subsection{Results and discussion}

Figure 3.2 shows the schematic illustration of the fabrication of micropillars on a base $p$-type wafer, radially doped with phosphorus. First, the backside of the wafer was covered with silicon nitride, which acts as a diffusion barrier to prevent the formation of a junction on the backside. The desired pattern of micropillars was transferred to the wafer using standard photolithography, and dry etching to achieve the desired height of the pillars. In order to fabricate a radial $\mathrm{p} / \mathrm{n}$ junction a phosphorus oxide was grown by SSD or CVD processes. Subsequently, a thermal step was done at a set temperature and time, to create a $p / n$ junction with the targeted junction depth. Finally, aluminum contacts were fabricated on the front and backside (upon removal of the SiRN layer) of the wafer to ensure an Ohmic contact to the silicon. In case of ridges and flat surfaces, the same procedure was followed, but then with larger dimensions and without any photolithographic pattern, respectively. 


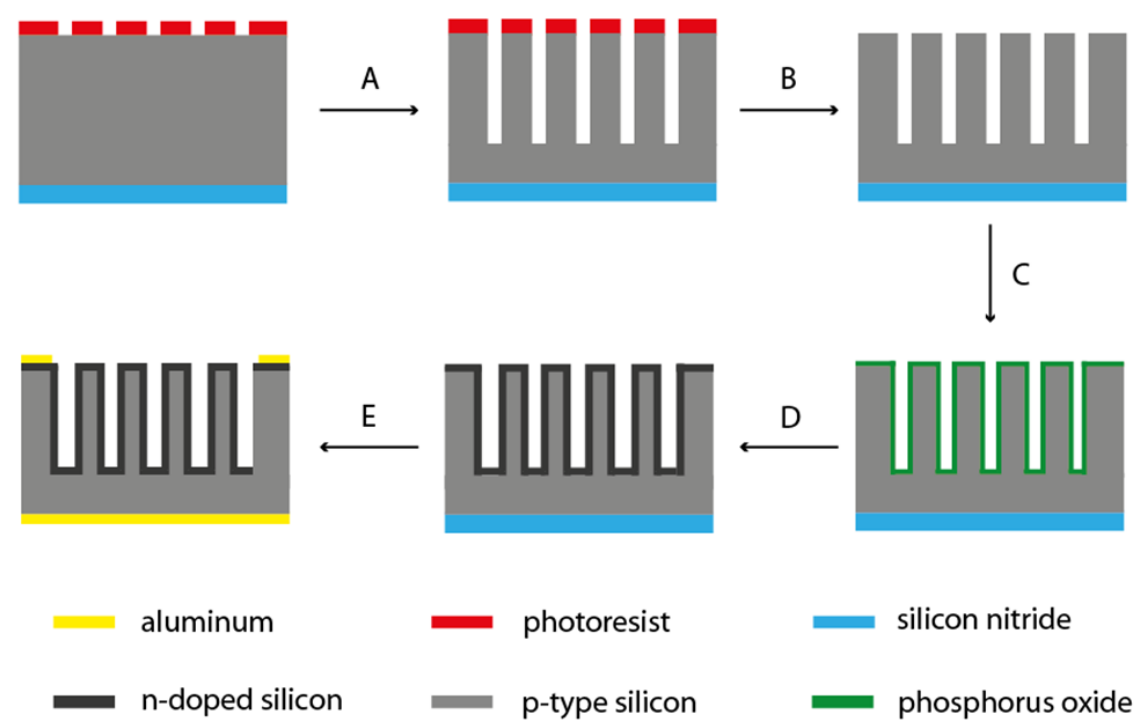

Figure 3.2: Schematic illustration of the fabrication of radial $p / n$ junctions in silicon micropillars. (A) Fabrication of silicon micropillars using DRIE on patterned photoresist on a silicon wafer. (B) Removal of residual photoresist by $\mathrm{O}_{2}$ plasma etching. (C) Formation of a phosphorus oxide layer, using CVD. (D) In-diffusion of phosphorus into the boron doped base wafer. (E) Removal of silicon nitride backside by HF etching, immediately followed by sputtering of the aluminum contacts. The same procedure was followed for boron doping of $n$-type silicon wafers, with SSD instead of CVD for the deposition of the dopant oxide layer.

\subsubsection{Junction analysis on flat substrates}

After the diffusion of the dopant, step $D$ in Figure 3.2, the $p / n$ junctions were analyzed by different methods. For flat surfaces, ball grooving and SIMS measurements were performed. Figure 3.3 shows typical grooves, under a light microscope, formed on flat doped surfaces by a stainless steel ball before $(A, B, C)$ and after $(D, E, F)$ staining. In case of an n-type silicon wafer that is boron-doped via SSD (Figure 3.3A, D), the inner part after staining (Figure 3.3D) shows the base n-type silicon and the gray-colored outer ring is the $p$-doped layer. Conversely, for $p$-type wafers that were doped with phosphorus by means of PECVD (Figure 3.3B, E) and LPCVD (Figure 3.3C, F), the inner part (base p-type) is darker than the outer ring (doped n-type), although this effect is less pronounced in the case of LPCVD doping. Diameters of both circles in the stained images were measured with 
image analysis software and junction depths (Table 3.1) were calculated by means of Equation 3.1. Thus, although the ball grooving and staining technique was originally developed for junctions with depths of $>10 \mu \mathrm{m}$, it also functions for junctions with a depth in the range of submicron to a few microns.

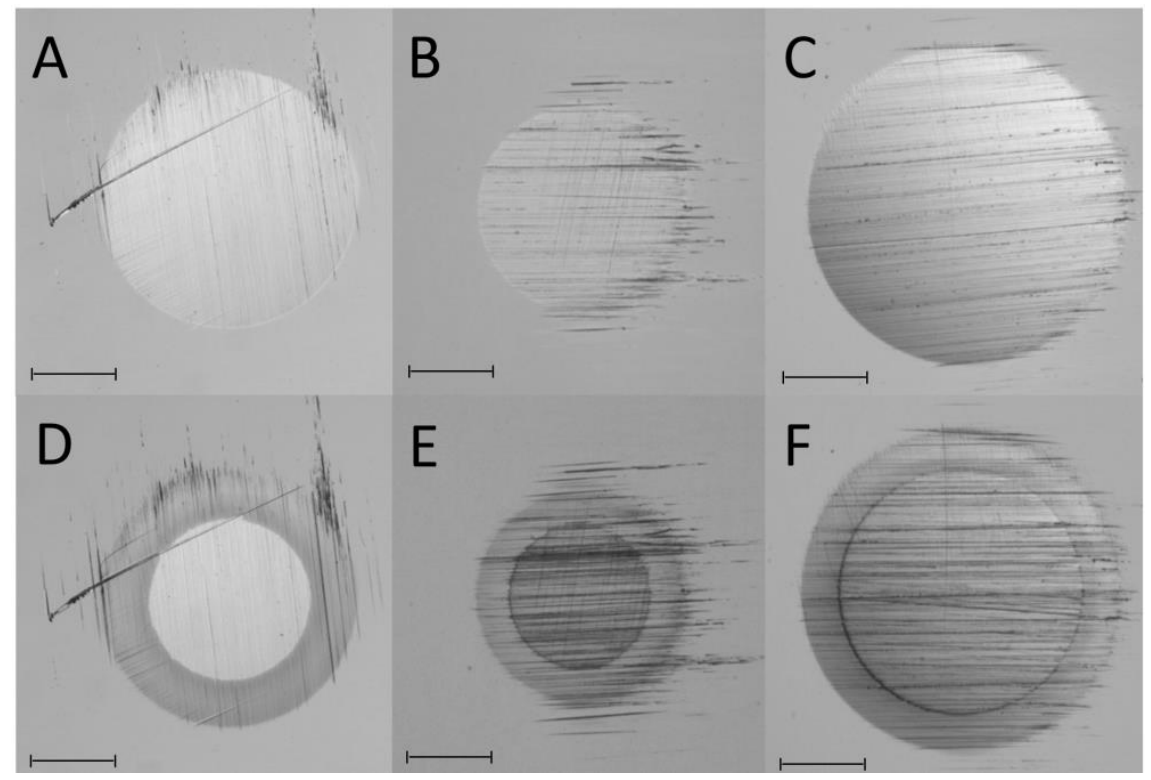

Figure 3.3: Optical microscopy images of flat, doped silicon samples after ball grooving, before $(A, B, C)$ or after (D, E, F) staining with CrO3/HF. (A, D) SSD-boron doped n-type silicon (1050 $\left.{ }^{\circ} \mathrm{C}, 15 \mathrm{~min}\right)$. (B, E) P-PECVD doped p-type silicon (1000 $\left.{ }^{\circ} \mathrm{C}, 15 \mathrm{~min}\right)$. (C, F) P-LPCVD doped p-type silicon (1050 $\left.{ }^{\circ} \mathrm{C}, 15 \mathrm{~min}\right)$. Scale bars represent $200 \mu \mathrm{m}$.

Table 3.1: Junction depth values of boron (SSD) and phosphorus (CVD) doped samples, based on data obtained from ball grooving and staining and from SIMS.

\begin{tabular}{lcc}
\hline & Staining $(\mu \mathrm{m})$ & SIMS $(\mu \mathrm{m})$ \\
\hline P-LPCVD $15 \mathrm{~min}, 1050^{\circ} \mathrm{C}$ & $1.1 \pm 0.1$ & $1.1 \pm 0.1$ \\
P-PECVD $15 \mathrm{~min}, 1050^{\circ} \mathrm{C}$ & $1.0 \pm 0.1$ & - \\
P-APCVD $15 \mathrm{~min}, 1050{ }^{\circ} \mathrm{C}$ & $2.8 \pm 0.1$ & $3.0 \pm 0.2$ \\
P-APCVD $100 \mathrm{~min}, 1000^{\circ} \mathrm{C}$ & $2.3 \pm 0.1$ & $2.4 \pm 0.2$ \\
B-SSD $15 \mathrm{~min}, 1000^{\circ} \mathrm{C}$ & $0.6 \pm 0.1$ & $0.7 \pm 0.1$ \\
B-SSD $15 \mathrm{~min}, 1050^{\circ} \mathrm{C}$ & $1.0 \pm 0.1$ & $1.3 \pm 0.1$ \\
B-SSD $120 \mathrm{~min}, 1050{ }^{\circ} \mathrm{C}$ & $2.2 \pm 0.1$ & $2.5 \pm 0.2$ \\
\hline
\end{tabular}


Figure 3.4 shows depth profiles of dopants as measured by SIMS on various flat-doped samples. As the base doping level of the silicon substrates was not measured with SIMS, but only derived from the resistivity, an accurate determination of the junction depth cannot be made.

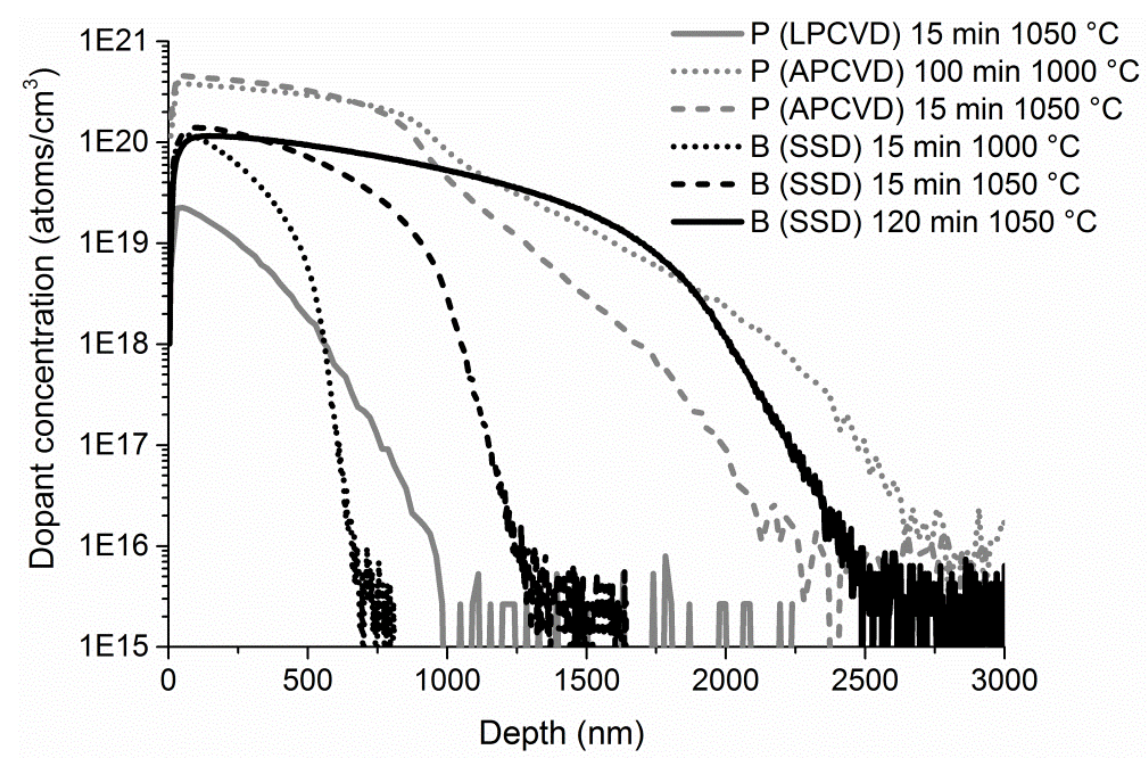

Figure 3.4: SIMS depth profiles of dopant elements (B/P) of flat, doped samples obtained at different in-diffusion temperature and time settings and using different doping processes $(P$ LPCVD, P-APCVD and B-SSD). The marked area is an example of the linear regime, used to extrapolate a more accurate junction depth, for boron doped (SSD) at $1050^{\circ} \mathrm{C}$ for $15 \mathrm{~min}$.

The base doping level varies from $5 \times 10^{14}$ to $5 \times 10^{15}$ atoms $/ \mathrm{cm}^{3}$ which is in the range of the measurement limitations of the SIMS system. Therefore an extrapolation over the linear regime (as indicated in Figure 3.4) was used to estimate the junction depths (Table 3.1), assuming an average doping level of $10^{15}$ atoms $/ \mathrm{cm}^{3}$. The differences in junction depths as determined with the staining method and the SIMS method are small, about 0.1-0.3 $\mu \mathrm{m}$, where SIMS indicates generally somewhat deeper junctions. Nevertheless, the observed trends in junction depth as function of drive-in temperature and time are identical for both methods. 
When comparing the different doping methods for phosphorus, the junction depths of the APCVD doped samples differed significantly from the other doping techniques. For the same time and temperature settings (15 min, $1050^{\circ} \mathrm{C}$ ), APCVD yielded a junction twice as deep as for LPCVD (2.3 vs. $1.1 \mu \mathrm{m}$ ). The SIMS profiles (Figure 3.4) give additional insight in the different doping mechanisms. For P-APCVD, the dopant concentration profiles show a bend at about $1 \mu \mathrm{m}$, and the surface concentrations exceed $2 \times 10^{20}$ atoms $/ \mathrm{cm}^{3}$. This is not the case for P-LPCVD and B-SSD doping, for which the diffusion profiles decrease monotonously.

In Figure 3.5, the experimentally determined junction depths are plotted (symbols) for three different temperatures and various doping techniques. Each symbol is an average over at least 5 measurements (ball grooving). Moreover, FEM simulation results, assuming simple Fick's law diffusion, are also given in Figure 3.5 (solid lines).

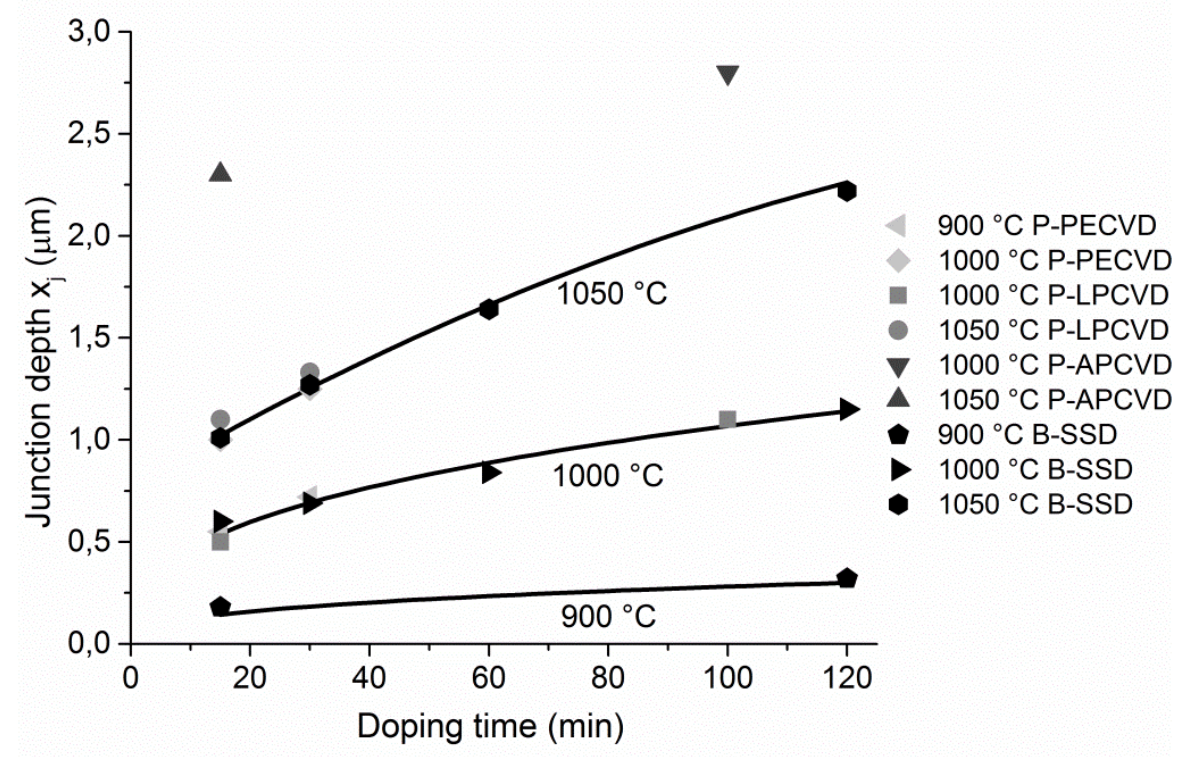

Figure 3.5: Junction depth as a function of drive-in time and temperature: solid lines represent simulations and symbols represent experimental data by means of ball grooving and staining. Error bars are not shown; the largest standard deviation was $0.09 \mu \mathrm{m}$. 
Clearly, most of the data points agree with the FEM simulations, except, as expected, the phosphorus-based junctions realized by APCVD. In fact, the junction depths obtained for this doping method are significantly larger than predicted by modeling and those found experimentally using other doping methods. This can be attributed to the high surface concentration of this doping method. High (surface) concentrations of phosphorus can give rise to anomalous kink-and-tail depth-diffusion profiles with a plateau region near the surface. ${ }^{[26]}$ In case of sufficiently high $\mathrm{P}$ surface concentrations $\left(>2 \times 10^{20}\right.$ atoms $/ \mathrm{cm}^{3}$ at a drive-in temperature of $\left.1000{ }^{\circ} \mathrm{C}\right)$, the so-called vacancy mechanism governs the dopant diffusion in the plateau region (at depths up to $\sim 1 \mu \mathrm{m}$ ), while the kick-out mechanism governs it in the deeper regions. In other words, diffusion of self-interstitials - also named point defects - dominates in the kink region, and $\mathrm{P}$ interstitials in the tail region. Only at high $P$ concentrations, the vacancy mechanism contributes to $P$ diffusion (and thereby enhances the overall dopant diffusion speed). The changeover from the vacancy mechanism to the kick-out mechanism is responsible for the appearance of the kink-and-tail depth-diffusion profiles visible in Figure 3.4 (APCVD data). In contrast, for low $P$ surface concentrations, only the kick-out mechanism affects the depth-diffusion profiles, and no plateau appears. In this case, Fick's diffusion law is valid as a model for $\mathrm{P}$ (and $\mathrm{B}$ ) diffusion into silicon, as shown for the investigated PECVD, LPCVD, and SSD settings. Due to the anomalous diffusion mechanism in the case of P-APCVD doping, this method is excluded from further analysis.

\subsubsection{Junction analysis in structured substrates}

$3 \mathrm{D}$ ridge and pillar structures, boron-doped using SSD $\left(1050{ }^{\circ} \mathrm{C}\right.$ for $120 \mathrm{~min}$ ), were investigated subsequently. Figure 3.6A and B show HR-SEM images of cross sections of ridge samples after staining with $\mathrm{CrO}_{3} / \mathrm{HF}$. Similar to staining on flat-doped surfaces, the staining is clearly visible in terms of a line on the junction interface. The junction depth of $2.2 \mu \mathrm{m}$ agrees with FEM simulations (Figure 3.6C) and with the staining experiments on flat surfaces (see above). 
The latter indicates that ball grooving of flat surfaces suffices to get a good indication of the junction depth also of micron-sized 3D structures. The sharp edge on the top (convex corner Figure 3.6B) and the round shape in the bottom corner (concave corner Figure 3.6C) are also present in the simulated ridge (Figure 3.6A). The images and simulations clearly indicate that boronSSD on 3D structures yields a uniform thickness of the doped layer.
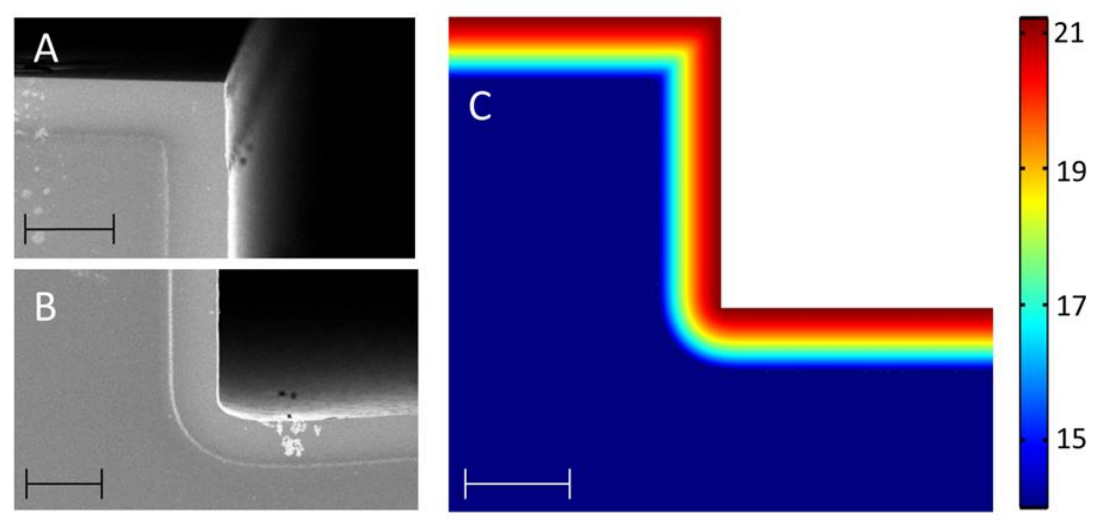

Figure 3.6: (A, B) Cross-sectional HR-SEM images $\left(80^{\circ}\right.$ angle) of $100 \times 30 \mu \mathrm{m}$ ridges (SSD-boron doped; $1050^{\circ} \mathrm{C}, 120 \mathrm{~min}$ ). Scale bars represent $3 \mu \mathrm{m}$. (C) Cross section of FEM simulated doping (SSD-boron doped; $1050^{\circ} \mathrm{C}, 120 \mathrm{~min}$ ) of a ridge structure.

Subsequently, the staining method was applied to micropillar structures. Two different approaches were used to stain the interior of pillars. In the first approach, the top of doped pillars was removed by means of a maskless DRIE step, as shown in Figure 3.7C. This enables a top view on such "chopped" pillars with SEM imaging (Figure 3.7A). Chopped pillars were also exposed to the staining solution, resulting in the appearance of a clear line on the inside of the pillar, as shown in Figure 3.7B, which resembles the $p / n$ interface. The second approach employed FIB etching to laterally remove half of a pillar. This enabled visualization of the junction along the height of a pillar (Figure 3.7D). 

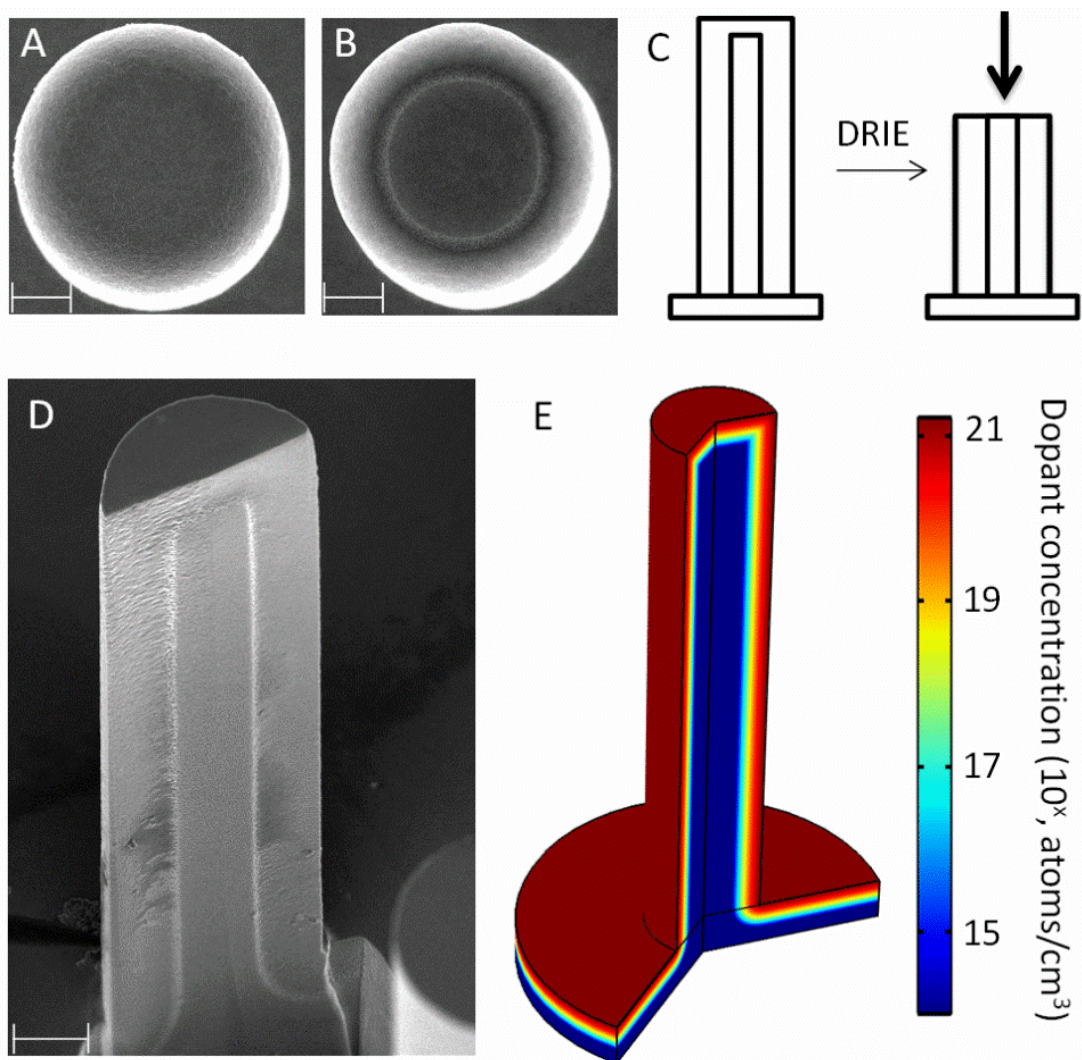

Figure 3.7: (A, B) Top view SEM images of unstained (A) and stained (B) pillars (boron doped SSD at $1050^{\circ} \mathrm{C}, 120 \mathrm{~min}$ ). (C) Schematic view of maskless DRIE etching in a pillar (cross section), to reveal the interior of the pillar. The thick arrow indicates the imaging direction of $A$ and B. (D) Side view of a split (using FIB) and stained pillar. (E) FEM simulation of a pillar with similar dimensions $(4 \mu \mathrm{m}$ width, $20 \mu \mathrm{m}$ height, boron doped at $\left.1050^{\circ} \mathrm{C}, 15 \mathrm{~min}\right)$. Scale bars represent $2 \mu \mathrm{m}$.

In case of the first approach - pillar chopping and staining (Figure 3.7A, B) the stained pillar clearly reveals the junction and junction depth $(2 \mu \mathrm{m})$. This stained line was also observed for the second approach, in which a pillar was cleaved vertically. As expected, the staining line perfectly follows the contour of the pillar: a uniform thickness $(2 \mu \mathrm{m})$ of the doped layer along the pillar circumference can be seen. The difference in surface roughness at the FIB interface visible between the left-hand and right-hand side of the cleaved pillar in Figure 3.7D is an artifact from a second FIB step. 
The seemingly thinner junction at the top side of the pillar is merely a result of the large angle at which the image was taken. Junction depths as determined with both approaches are in agreement with previous measurements on flat samples (see above) and FEM simulations (Figure 3.7E, $2.2 \mu \mathrm{m}$ ).

Similar results, regarding uniform doping along the pillar height, were obtained for P-LPCVD doped samples. In the case of P-PECVD doped pillars, no contrast was visible along the pillar height, which is believed to be caused by directionality during formation of the dopant layer. For this reason, the P-PECVD samples were also excluded in the JV measurements.

\subsubsection{Electrical characterization}

In order to verify the influence of radial $\mathrm{p} / \mathrm{n}$ junctions on light capturing capabilities, JV measurements were performed on doped flat surfaces and similarly radially doped micropillar arrays, i.e. SSD-boron on n-type silicon and LPCVD-phosphorus on p-type silicon $\left(1050^{\circ} \mathrm{C}, 15 \mathrm{~min}\right) . J V$ plots are shown in Figure 3.8 . 


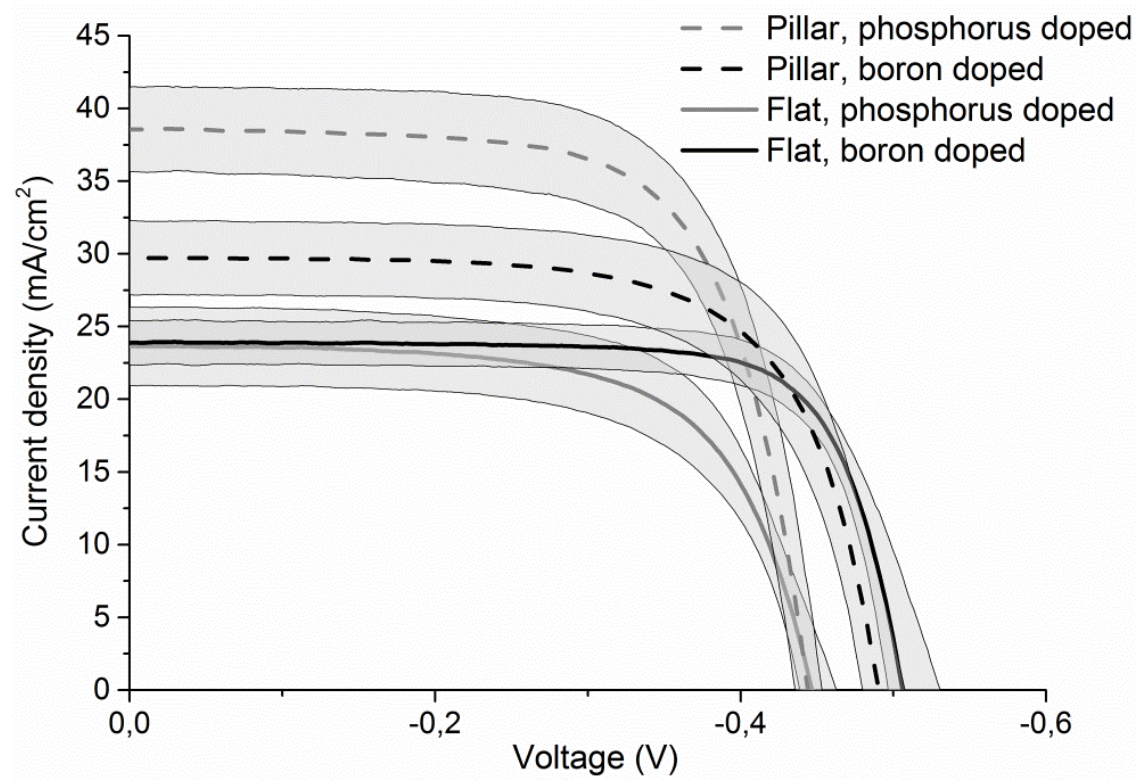

Figure 3.8: $J V$ measurements of different samples: flat junctions (continuous lines) and radial junctions in pillar arrays (dashed lines) for boron and phosphorus dopants. The dotted lines indicate the $1 \sigma$-range around the average (at least 5 samples were analyzed for each configuration). The current density is normalized to the sample area (not the actual surface area of the pillars).

The open-circuit voltage $\left(V_{O C}\right)$ for boron-doped samples is approximately $0.5 \mathrm{~V}$, whereas for phosphorus doped samples the $V_{O C}$ is around $0.45 \mathrm{~V}$, which is slightly lower than the $0.5-0.7 \mathrm{~V}$ reported in literature for silicon. ${ }^{[1,2,27]}$ It has to be noted that, in contrast to many literature studies, neither back reflector nor surface passivation was applied in our work. For both boron and phosphorus doping, the short-circuit current density $\left(J_{S C}\right)$ values were significantly higher for pillar samples -30 and $38 \mathrm{~mA} / \mathrm{cm}^{2}$ for boron and phosphorus doping, respectively - compared to doped flat samples (ca. $\left.24 \mathrm{~mA} / \mathrm{cm}^{2}\right)$.

Using the fill factor $(F F), V_{O C}$ and $J_{S C}$, the overall efficiency $\eta$ can be calculated (Equation 3.2):

$$
\eta=\frac{V_{O C^{*} J_{S C} * F F}}{P_{\text {in }}}
$$

where $P_{\text {in }}$ is the input power, which is $100 \mathrm{~mW} / \mathrm{cm}^{2}$ (AM 1.5). 
For boron-doped samples, the efficiency increased from $8.3 \%$ for flat samples to $9.4 \%$ for pillar arrays, whereas phosphorus-doped samples showed $\eta$ values of $6.4 \%$ and $11.0 \%$ for flat and pillared samples, respectively. These efficiencies for radially doped $\mathrm{p} / \mathrm{n}$ junctions are in agreement with literature values, typically showing efficiencies above $5 \% .^{[1,2,19,27,28]}$ Thus, properly doped radial $p / n$ junctions indeed enhance the light trapping via an increase in effective junction area on a given footprint $\left(0.5 \times 0.5 \mathrm{~cm}^{2}\right)$.

A pillar array with a junction depth larger than $2 \mu \mathrm{m}$ (SSD-boron of n-type silicon, drive-in settings: $1050^{\circ} \mathrm{C} / 120 \mathrm{~min}$ ) was also subjected to $\mathrm{JV}$ analysis. In this case, the junction depth is larger than the pillar radius, leading to completely doped-through pillars. Such over doped pillar arrays displayed a low $J_{S C}$ value $\left(7 \mathrm{~mA} / \mathrm{cm}^{2}\right)$, which can be attributed to a loss of effective junction area on the $0.5 \times 0.5 \mathrm{~cm}^{2}$ footprint: $35 \%$ of the sample footprint is covered with pillars. Although the pillars themselves do lower the reflectivity of the sample, this is apparently not sufficient to compensate for the loss in effective junction area. As expected, over-doped pillars showed a poor efficiency (2\%). Altogether, these results show the potential of using radially doped micropillars for more efficient light capturing, at the same time emphasizing the need for proper control of the doping process to achieve the appropriate junction depth.

\subsection{Conclusions}

All investigated doping methods, i.e. SSD, LPCVD, APCVD, PECVD, gave uniform $\mathrm{p} / \mathrm{n}$ junctions on horizontal/flat surfaces. SSD and LPCVD also yielded homogeneous junction depths on 3D structures (i.e. microridges, micropillars) in silicon. Ball grooving and staining on flat surfaces yielded accurate values for junction depths, and the values are similar to data based on HR-SEM (on flat and $3 D$ samples) and SIMS. Junctions made by doping using B-SSD, P-LPCVD or P-PECVD can be accurately predicted using Fick's law. In case of P-APCVD junctions, the measured dopant concentration profiles (and hence junction depths) deviated from model results, typically resulting in much 
deeper junctions. This is attributed to an additional diffusion mechanism (i.e. vacancy mechanism).

Radial junctions made by SSD (boron) and LPCVD (phosphorus) doping had higher $J_{S C}$ values and efficiencies compared to doped flat surfaces. The positive effect on light capturing by arrays of properly doped radial junctions was further evidenced with experiments on over-doped pillar arrays, which displayed an even lower $J_{S C}$ than doped flat substrates.

Future experiments will focus on the effect of the junction depth in micropillar arrays and footprint size on the light capturing efficiency. Moreover, such pillar arrays with radial junctions will be implemented in solar-to-fuel devices.

\section{Acknowledgements}

Roald Tiggelaar is acknowledged for the assistance with the experiments and the discussions. Alexander Milbrat assisted with the JV setup and measurements. Mark Smithers is acknowledged for the HR-SEM images, Frans Segerink is thanked for the FIB experiments. 


\subsection{References}

[1] E. Garnett, P. Yang, Nano Lett., 2010, 10, 1082-1087.

[2] M. C. Putnam, S. W. Boettcher, M. D. Kelzenberg, D. B. Turner-Evans, J. M. Spurgeon, E. L. Warren, R. M. Briggs, N. S. Lewis, H. A. Atwater, Energy Environ. Sci., 2010, 3, 1037-1041.

[3] K. Q. Peng, S. T. Lee, Adv. Mater., 2011, 23, 198-215.

[4] V. Schmidt, J. V. Wittemann, U. Gösele, Chem. Rev., 2010, 110, 361-388.

[5] R. Kapadia, Z. Fan, K. Takei, A. Javey, Nano Energy, 2012, 1, 132-144.

[6] M. D. Kelzenberg, D. B. Turner-Evans, B. M. Kayes, M. A. Filler, M. C. Putnam, N. S. Lewis, H. A. Atwater, Nano Lett., 2008, 8, 710-714.

[7] M. Gharghi, E. Fathi, B. Kante, S. Sivoththaman, X. Zhang, Nano Lett., 2012, 12, 62786282.

[8] E. L. Warren, H. A. Atwater, N. S. Lewis, J. Phys. Chem. C, 2013, 118, 747-759.

[9] A. I. Hochbaum, P. Yang, Chem. Rev., 2009, 110, 527-546.

[10] I. Oh, J. Kye, S. Hwang, Nano Lett., 2011, 12, 298-302.

[11] Y. Hou, B. L. Abrams, P. C. K. Vesborg, M. E. Björketun, K. Herbst, L. Bech, A. M. Setti, C. D. Damsgaard, T. Pedersen, O. Hansen, J. Rossmeisl, S. Dahl, J. K. Nørskov, I. Chorkendorff, Nat. Mater., 2011, 10, 434-438.

[12] S. W. Boettcher, E. L. Warren, M. C. Putnam, E. A. Santori, D. Turner-Evans, M. D. Kelzenberg, M. G. Walter, J. R. McKone, B. S. Brunschwig, H. A. Atwater, N. S. Lewis, J. Am. Chem. Soc., 2011, 133, 1216-1219.

[13] E. L. Warren, J. R. McKone, H. A. Atwater, H. B. Gray, N. S. Lewis, Energy Environ. Sci., 2012, 5, 9653-9661.

[14] S. Yu, F. Roemer, B. Witzigmann, J. Photon Energy, 2012, 2, 0280021-0280029.

[15] A. Mallorquí, E. Alarcón-Lladó, I. Mundet, A. Kiani, B. Demaurex, S. De Wolf, A. Menzel, M. Zacharias, A. Fontcuberta i Morral, Nano Res., 2014, 1-9.

[16] A. C. Fischer, L. M. Belova, Y. G. M. Rikers, B. G. Malm, H. H. Radamson, M. Kolahdouz, K. B. Gylfason, G. Stemme, F. Niklaus, Adv. Funct. Mater., 2012, 22, 40044008.

[17] S. Ingole, P. Aella, P. Manandhar, S. B. Chikkannanavar, E. A. Akhadov, D. J. Smith, S. T. Picraux, J. Appl. Phys., 2008, 103, 104302-104308.

[18] J. C. Ho, R. Yerushalmi, Z. A. Jacobson, Z. Fan, R. L. Alley, A. Javey, Nat. Mater., 2008, 7, 62-67.

[19] J. Jin-Young, G. Zhongyi, J. Sang-Won, U. Han-Don, P. Kwang-Tae, H. Moon Seop, Y. Jun Mo, L. Jung-Ho, Nanotechnology, 2010, 21, 445303.

[20] B. S. Meyerson, W. Olbricht, J. Electrochem. Soc., 1984, 131, 2361-2365.

[21] L. Yu, S. Misra, J. Wang, S. Qian, M. Foldyna, J. Xu, Y. Shi, E. Johnson, P. R. i. Cabarrocas, Sci. Rep., 2014, 4, 4357.

[22] P. L. O'Sullivan, F. H. Baumann, G. H. Gilmer, J. Appl. Phys., 2000, 88, 4061-4068.

[23] U.-H. Kwon, W.-J. Lee, Thin Solid Films, 2003, 445, 80-89.

[24] R. S. Muller, T. I. Kamins, M. Chan, Device Electronics for Integrated Circuits, John Wiley \& Sons Australia, Limited, 2003.

[25] W.C. Roman, L.R. Wilson, US Patent 3.830.665, 1974.

[26] M. Uematsu, J. Appl. Phys., 1997, 82, 2228-2246.

[27] D. R. Kim, C. H. Lee, P. M. Rao, I. S. Cho, X. Zheng, Nano Lett., 2011, 11, 2704-2708.

[28] A. Dalmau Mallorquí, F. M. Epple, D. Fan, O. Demichel, A. Fontcuberta i Morral, Phys. Status Solidi A, 2012, 209, 1588-1591. 


\section{Chapter 4}

\section{Effects of Pillar Height and Junction Depth on the Performance of Radially Doped Silicon Pillar Arrays for Solar Energy Applications}

The effects of pillar height and junction depth on solar cell characteristics were investigated in order to provide design rules for arrays of such pillars in solar energy applications. Radially doped silicon pillar arrays were fabricated by deep reactive ion etching (DRIE) of silicon substrates followed by the introduction of dopant atoms by diffusion from a phosphorus oxide deposited by low-pressure chemical vapor deposition. Increasing the height of the pillars led to doubling of the efficiency from 6\% for flat substrates to $12 \%$ for $40 \mu \mathrm{m}$ high pillars (at $900 \mathrm{~nm}$ junction depth), because of an increase of the total junction area and lower optical reflection. For higher pillars, the current density and efficiency decreased, which is attributed to the presence of larger amounts of defect states at the surface introduced during the etching process. This effect could be counteracted by a passivation layer $\left(\mathrm{Al}_{2} \mathrm{O}_{3}\right)$ on the pillar surface. Junction depths below $600 \mathrm{~nm}$ resulted in leaky diodes. An optimum in efficiency (13\%) was found for a junction depth of $790 \mathrm{~nm}$ (at $40 \mu \mathrm{m}$ pillar height). At increased junction depths, the efficiency decreased due to thinning of the undoped core of the pillars, which was supported by the observation that pillars with a diameter of $4 \mu \mathrm{m}$ and a junction depth of $1.2 \mu \mathrm{m}$ became less efficient than flat silicon substrates.

This chapter has been published as: R. Elbersen, W.J.C Vijselaar, R.M. Tiggelaar, J.G.E. Gardeniers, J. Huskens, Adv. Energy Mater., 2015, accepted 


\subsection{Introduction}

Silicon is a widely used material in the field of photovoltaics and solar-to-fuel devices, because of its outstanding electrical properties and the large toolbox available for structuring of the material. ${ }^{[1-3]}$ Combined with the earth abundancy and relatively low production costs, it is used extensively in the nano/micro-fabrication area. Previous studies in the field of photovoltaics have shown large improvement in solar cell performance, such as a lower reflectivity of silicon, structuring by means of wet and dry etching as well as various methods to introduce dopants. ${ }^{[4-7]}$ One of the recent developments is the shift from planar to radial $\mathrm{p} / \mathrm{n}$-junctions using nano- or micropillars. ${ }^{[8,9]}$ The advantage of pillars with a radial $p-n$ junction for use in solar panels, and more specifically in solar-to-fuel devices, is two-fold. First, the radial junction decouples the direction of light incidence and charge transport processes, reducing the recombination losses. Secondly, the high surface area for a given footprint is ideal for catalysis and light absorption, as a lower reflectivity results in improved light absorption. ${ }^{[10-12]}$ Already a few examples of full-scale (lab) devices for solar-to-fuel have been reported, where hydrogen is produced in an electrolyte using a silicon solar cell functionalized with proper catalysts. ${ }^{[13,14]}$ Optimization of the silicon solar cell is important for both photovoltaics and solar-to-fuel applications, since higher efficiencies will ultimately affect their economic benefit and thereby the commercial and industrial interest in solar energy devices.

Pillar dimensions (height, width and density) and $p / n$ junctions in the pillars (radial vs. axial, depth and doping levels) have effects on all performance parameters discussed above (light absorption, charge separation and surface area) which ultimately determine device efficiency. Yet, the relationships between these fabrication and performance aspects are difficult to predict and decouple. For example, increasing the height of the pillars inherently increases the total junction area present in the solar cell, and possibly decreases the reflectivity of sunlight. ${ }^{[15]}$ Previous publications have shown a positive effect of 
increased pillar heights on the efficiency of solar cells. ${ }^{[16,17]}$ Voight et al. have used simulations to predict the most efficient radius and height of a pillar. ${ }^{[16]}$ In summary, they found an optimum of $2 \mu \mathrm{m}$ for the pillar diameter with a corresponding height of $96 \mu \mathrm{m}$. Another important aspect in the design of the pillar array is the diameter-over-pitch (D/P) ratio. A D/P ratio between 0.5-0.8 has been suggested, as this has shown the highest theoretical and experimental efficiencies for various systems. ${ }^{[17-19]}$ The radial junction depth variation in micropillars can be varied by tuning the doping process, and a trend of improved $J V$ characteristics for thinner junctions, down to $50 \mathrm{~nm}$, has been shown in simulations. ${ }^{[18]} A$ thin junction leads to less recombination, which implies that the outer highly doped shell of a radially doped micropillar should be kept thin, but also should keep its carrier separation functionality. Pillars with a deep junction may suffer from bulk recombination, which negatively affects the efficiency of the solar cell. ${ }^{[20]}$ Clearly, an optimal radial junction depth exists for any given configuration of a solar cell (i.e. pillar diameter, pitch, height and specimen footprint) that yields the highest overall efficiency. To date, no studies have shown the joint optimization of pillar height and junction depth.

Here, we show the optimization of the electrical performance of radially doped arrays of micropillars with a fixed diameter, pitch and footprint by variation of the pillar height and junction depth $\left(x_{j}\right)$. Arrays were used with a pillar diameter of $4 \mu \mathrm{m}$ in combination with a pitch of $6 \mu \mathrm{m}(\mathrm{D} / \mathrm{P}=0.67)$, and heights in the range of 0 to $60 \mu \mathrm{m}$. At the same time, a full range of junction depths, from shallow ( 140 nm) to deep junctions ( 1640 nm), has been realized using lowpressure chemical vapor deposition (LPCVD) for the creation of the required dopant source, i.e. a thin layer of phosphorus oxide. Such a sweep of different junction depths can lead to the optimization of the solar cell, for a given doping technique. Furthermore, preliminary experiments are discussed to further improve the efficiency of pillar array-based solar cells by depositing a passivation coating on the surface of the micropillars. 


\subsection{Materials and methods}

\subsubsection{Fabrication of radial $\mathrm{p} / \mathrm{n}$ junctions in silicon micropillar arrays}

Radial $\mathrm{p} / \mathrm{n}$ junctions were prepared as previously reported, and shown in Figure 4.1. ${ }^{[7]}$ In short, p-type silicon substrates ((100) oriented, 5-10 $\Omega \mathrm{cm}, 525$ $\mu \mathrm{m}$ thickness, single side polished, Okmetic Finland) were covered with 100 $\mathrm{nm}$ silicon nitride $\left(\mathrm{SiN}_{\mathrm{x}}\right)$. On the front side, after removal of the $\operatorname{SiN}_{\mathrm{x}}$, the substrate was patterned with arrays of hexagonally packed dots $(4 \mu \mathrm{m}$ diameter, $6 \mu \mathrm{m}$ pitch, $0.5 \times 0.5 \mathrm{~cm}^{2}$ cell size on a specimen of $2 \times 2 \mathrm{~cm}^{2}$ ) by standard photolithography (Olin 907-17 photoresist). This pattern was transferred into the silicon by deep reactive ion etching (CF-chemistry, Adixen AMS100SE), and the height of the pillar arrays was controlled by the etching time, whereby the etch rate of silicon was ca. $3.2 \mu \mathrm{m} / \mathrm{min}$. After removal of the photoresist layer and residual fluorocarbons using an oxygen plasma, a phosphorus oxide (approximately $400 \mathrm{~nm}$ thick) was grown by LPCVD (Tempress tube furnace), using phosphine $\left(\mathrm{PH}_{3}\right)$ as a dopant precursor. Dopant transfer into the pillars was performed at temperatures ranging from 900 to $1100^{\circ} \mathrm{C}$ and various drive-in times (5-60 min) under a stream of $\mathrm{N}_{2}$. The combination of drive-in time and temperature determines the radial junction depth in the pillars. After the thermal diffusion step, the phosphorus oxide was removed by immersion in BHF ( $\mathrm{NH}_{4} \mathrm{~F}$-buffered aqueous $\mathrm{HF}$ ) for $10 \mathrm{~min}$, as well as stripping off the silicon nitride from the backside of the substrate in $50 \%$ aqueous HF (30 min). Values of the junction depth were predicted by FEM (Finite Element Modeling, COMSOL Multiphysics 4.2) simulations and verified with ball grooving and staining. ${ }^{[7]}$ 

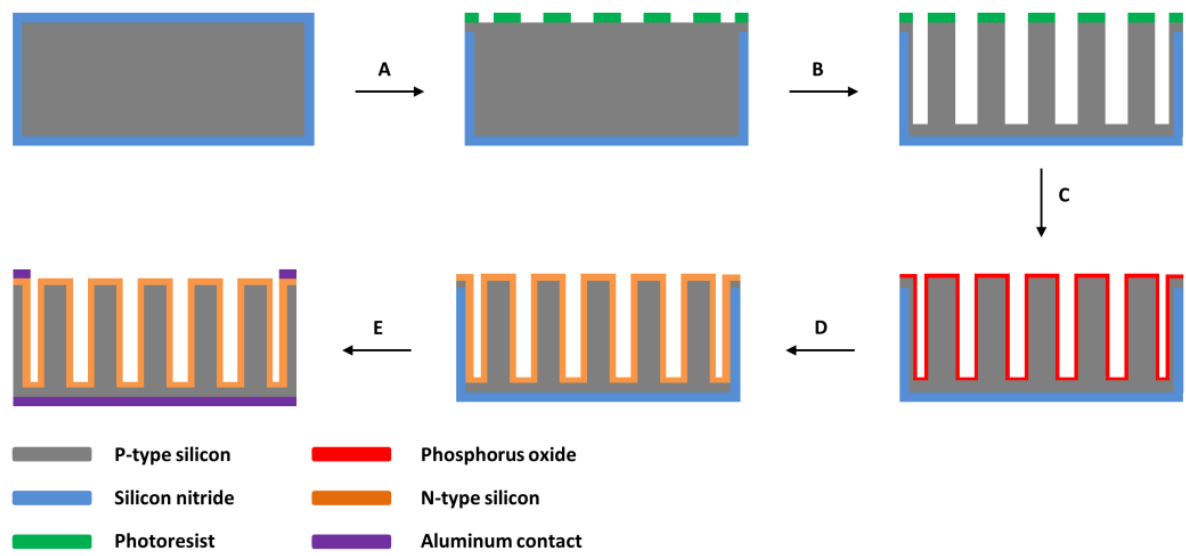

Figure 4.1: Schematic fabrication process. (A) Removal of topside silicon nitride, followed by photolithography. (B) Deep reactive ion etching of $\mathrm{Si}$. (C) Low-pressure chemical vapor deposition of phosphorus oxide. (D). Drive-in diffusion of phosphorus, resulting in radial $\mathrm{p} / \mathrm{n}$ junctions. (E) Removal of silicon nitride and deposition of metal front and back contact.

\subsubsection{Atomic layer deposition}

To reduce the reflectivity of the micropillar arrays, a layer $(42 \mathrm{~nm})$ of aluminum oxide $\left(\mathrm{Al}_{2} \mathrm{O}_{3}\right)$ was conformally coated on several samples, using a thermal ALD process at $100{ }^{\circ} \mathrm{C}$ (Picosun P-300S reactor). Trimethyl-aluminum (TMA) and $\mathrm{H}_{2} \mathrm{O}$ were used as precursors and $\mathrm{N}_{2}$ was the carrier gas, yielding a growth rate of $0.83 \AA$ per cycle (after initial growth stabilization). The time for one complete $\mathrm{Al}_{2} \mathrm{O}_{3}$ ALD cycle was $9.3 \mathrm{~s}$, where each cycle was defined as: $0.1 \mathrm{~s}$ TMA exposure, $4 \mathrm{~s} \mathrm{~N}_{2}$ purge, $0.2 \mathrm{~s}_{2} \mathrm{O}$ exposure, and $5 \mathrm{~s} \mathrm{~N}_{2}$ purge.

\subsubsection{JV measurements}

To ensure Ohmic contact to the solar cells, $1 \mu \mathrm{m}$ aluminum/silicon alloy (99\% Al, 1\% Si) was sputtered (Oxford PL 400) on the front and backside of each specimen, where on the front side a shadow mask was applied to protect the pillar arrays from coating. To measure the photovoltaic characteristics, the samples were positioned perpendicular to a $300 \mathrm{~W}$ xenon arc light source with an intensity of 1 sun (AMG 1.5). JV measurements were recorded on a VersaSTAT 4 potentiostat for a linear voltage sweep from -0.7 to $0.7 \mathrm{~V}$ at a rate of $0.2 \mathrm{~V} / \mathrm{s}$. For each setting (pillar height or junction depth) at least four different samples were measured. 


\subsection{Results and discussion}

After DRIE fabrication of the micropillars, a few samples were broken and the cross sections were taken in a scanning electron microscope (Figure 4.2). Due to the nature of the etching process (B-HARS) on high aspect ratio structures, a slight tapering occurred at about $33 \%$ from the top of the pillars. This effect was more pronounced for the $60 \mu \mathrm{m}$ pillars (18 min etching), where the diameter decreased approximately 500-600 nm, compared to $100-200 \mathrm{~nm}$ in the case of $40 \mu \mathrm{m}$ (12 min etching). The pillars were still broad enough to prevent an overdoping of the pillar arrays, and losing the beneficial effect of a radial $\mathrm{p} / \mathrm{n}$ junction, however it can potentially influence the measurements.

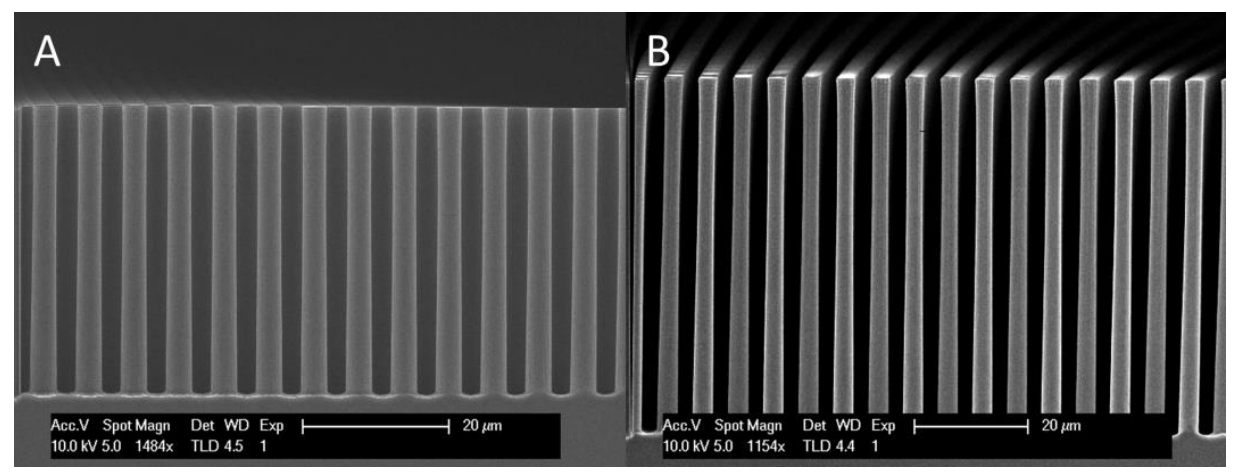

Figure 4.2: Cross-sectional SEM images of micropillar arrays with a height of $40 \mu \mathrm{m}(\mathrm{A})$ and $60 \mu \mathrm{m}(\mathrm{B})$.

The combinations of drive-in temperature and time used here led to junction depths between $140-1640 \mathrm{~nm}$, as predicted by FEM simulations, ${ }^{[7]}$ and were experimentally verified by ball grooving and staining experiments on five different flat samples $(140,790,900,1170$ and $1550 \mathrm{~nm}$, see Chapter 3 for methodology). Since the phosphorus surface concentration upon drive-in is relatively low, transport of phosphorus into silicon can be described by Fick's law of diffusion. ${ }^{[21]}$

The influence of the height of silicon micropillars with incorporated radial $p / n$ junctions was investigated by analyzing their electrical properties. Figure 4.3 shows the $J V$ characteristics of samples with seven different pillar heights, i.e. from flat to $60 \mu \mathrm{m}$. All samples had a junction depth of $900 \mathrm{~nm}$. The large 
increase in both current density $\left(J_{S C}\right)$ as well as open circuit voltage $\left(V_{O C}\right)$ for a sample with $10 \mu \mathrm{m}$ high pillars compared to a flat sample (Figure 4.3A) indicates that even a shallow structure already drastically improves the light absorbing properties of the solar cell. This can be explained by both an increase in total junction area and a decrease in reflectively. The latter is supported by previously reported work by various groups, who showed that the reflectivity of polished, flat silicon (ca. 35\%) decreases to values below $10 \%$ by even the smallest amount of micro/nano structuring. ${ }^{[1,22,23]}$ The parameters $J_{S C}$ and $V_{O C}$ were plotted as a function of the pillar height (Figure 4.3B), along with their respective fill factors $(F F)$ and efficiencies $(\eta)$, as shown in Figure 4.3C. A monotonous increase of $V_{O C}$ is visible, from $400 \mathrm{mV}$ for flat samples to $460 \mathrm{mV}$ for $60 \mu \mathrm{m}$ high pillars. This is attributed to the increase of the total surface area - and concomitantly of the junction area - of the solar cell on the given footprint, which is 5 times higher for $10 \mu \mathrm{m}$ high pillars and increases to 22 times for the $60 \mu \mathrm{m}$ high pillars. The $J_{S C}$ values, after a gradual increase with pillar height up to $40 \mu \mathrm{m}$, decrease again for larger pillar heights. This is reflected in the overall efficiency: since the fill factor remains fairly constant, the efficiency exhibits a maximum for a pillar height of $40 \mu \mathrm{m}$. We conclude that for these pillar arrays, with a $4 \mu \mathrm{m}$ diameter, $2 \mu \mathrm{m}$ spacing and a junction depth of $900 \mathrm{~nm}$, the optimum pillar height is $40 \mu \mathrm{m}$. 

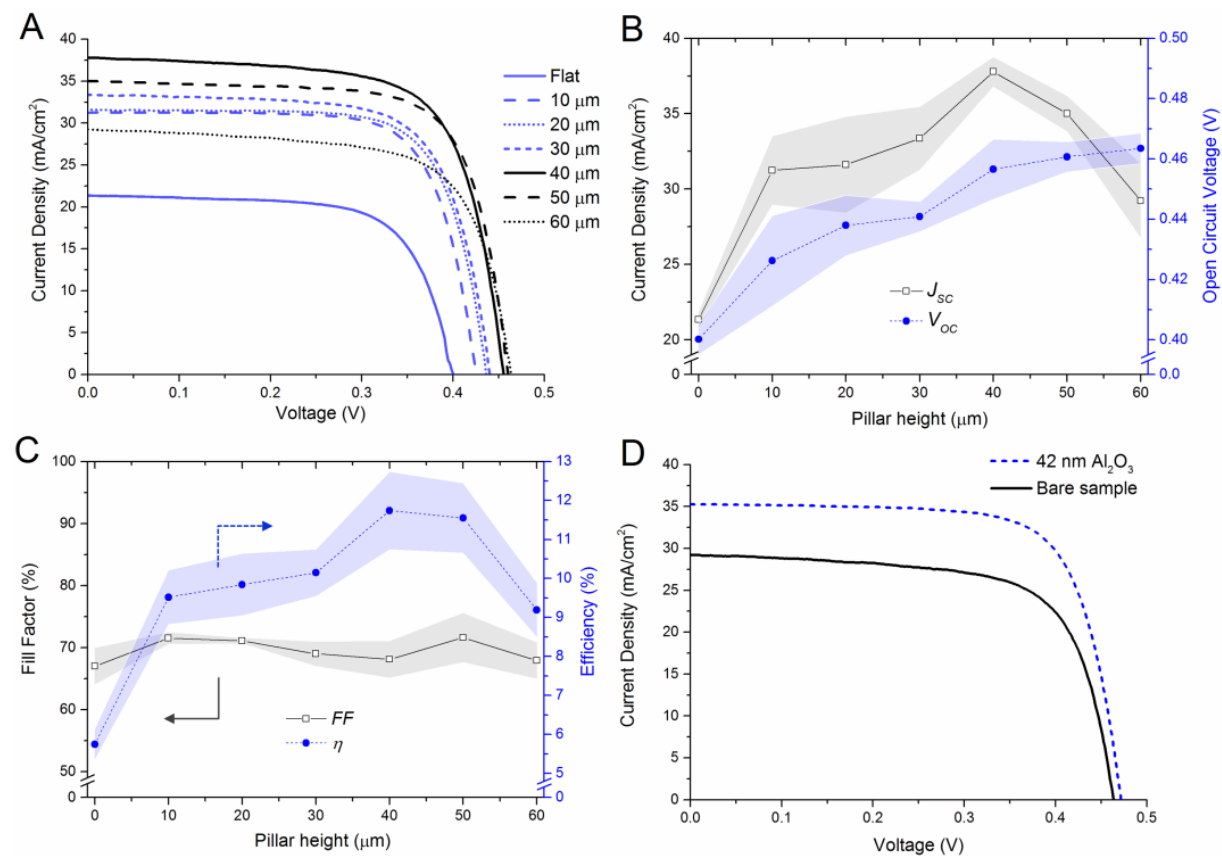

Figure 4.3: (A) JV measurements of n-emitter (900 nm junction depth) $\mathrm{p}$-type base substrates for different pillar heights, each line is the average of at least 4 different samples of the same setting. (B) Current density and open circuit voltage as a function of the pillar height, error bars indicate the 1 $\sigma$-range. (C) Corresponding fill factors and efficiencies of the average values shown in B. (D) $J V$ measurements on specimen with 60 $\mu \mathrm{m}$ high pillars before and after ALD deposition of $42 \mathrm{~nm} \mathrm{Al}_{2} \mathrm{O}_{3}$.

We attribute the decrease in current density for heights above $40 \mu \mathrm{m}$ to surface recombination by an increasing number of defect states at the, equally increasing, outer surface of the silicon pillars. These defects have been induced by the dry etching method in combination with the circular pillar design, because of which the sidewalls of the pillars are not terminated by a low-index crystallographic plane of silicon. This has led to defects at the surface of the silicon pillar compared to (100) silicon. ${ }^{[24]}$ In addition, the working principle of the applied 'Bosch' process, which is a cyclic process involving gas pulses of $\mathrm{SF}_{6}$ (etching gas) and $\mathrm{C}_{4} \mathrm{~F}_{8}$ (passivation gas), has led to scalloping of the pillar sides, ${ }^{[25]}$ which further increases the amount of defect states. ${ }^{[24]}$ Cryogenic etching would lead to pillars with a lower sidewall roughness without scallops, however, this method did not produce good quality micropillars with heights above $20 \mu \mathrm{m}$ at the pitch used here. 
To reduce surface recombination, a passivation layer (42 nm) of $\mathrm{Al}_{2} \mathrm{O}_{3}$ was deposited by atomic layer deposition (ALD) on a sample with $60 \mu \mathrm{m}$ pillars, after initial $J V$ analysis of the same specimen. The passivation layer resulted in increased $J_{S C}$ (Figure 4.3D), open circuit voltage and fill factor. The increase in $J_{S C}$ may also be explained by the anti-reflective properties of the $\mathrm{Al}_{2} \mathrm{O}_{3}$ layer. ${ }^{[26]}$ The $J V$ curves were fitted to determine the shunt $\left(R_{S H}\right)$ and series $\left(R_{S}\right)$ resistances, and the open circuit voltage $\left(V_{O C}\right)$. Upon application of the passivation layer, $R_{S H}$ was found to increase from 308 to $1064 \Omega \mathrm{cm}^{2}$ and $R_{S}$ decreased from 3.1 to $2.4 \Omega \mathrm{cm}^{2}$, whereas the $V_{O C}$ barely changed. These improvements show that surface defects indeed play an important role for arrays with higher pillar, and that a conformal coating of $\mathrm{Al}_{2} \mathrm{O}_{3}$ can be used to reduce the impact of the defects.

Subsequently, the junction depth was varied by changing the drive-in temperature between $900-1100^{\circ} \mathrm{C}$ and the drive-in time between 5-60 min using pillar arrays with the optimum pillar height of $40 \mu \mathrm{m}$. Figure 4.4 shows $\mathrm{JV}$ measurements for twelve different junction depths. Junctions made at 900 or $950{ }^{\circ} \mathrm{C}$ (see Figure $4.4 \mathrm{~A}$ ) did not give properly functioning diodes, most likely due to too low dopant concentrations $\left(<2 \times 10^{19}\right.$ atoms $/ \mathrm{cm}^{3}$ surface concentration for $1050^{\circ} \mathrm{C}$ as determined by SIMS, see Figure 4.5). In case of a drive-in temperature of $1000{ }^{\circ} \mathrm{C}$, both the series and shunt resistances decreased significantly with the drive-in time, resulting in a proper diode (Figure 4.4A). As a consequence, higher current densities and correspondingly increased open circuit voltages were observed. The increase in $J_{S C}$ and $V_{O C}$ for thicker junctions continued until a junction depth of $790 \mathrm{~nm}$ (Figure 4.4B). For junctions $\geq 90 \mathrm{~nm}$ the current density dropped, and the $V_{O C}$ also decreased slightly, but not as pronounced as $J_{S C}$ (Figure 4.4C). The series and shunt resistances remained rather constant for larger junction depths, due to which the FF remained constant as well at about 70\% (Figure 4.4D). For $x_{j}$ values $\geq 790 \mathrm{~nm}$ bulk recombination became significant, resulting in lower efficiencies. 

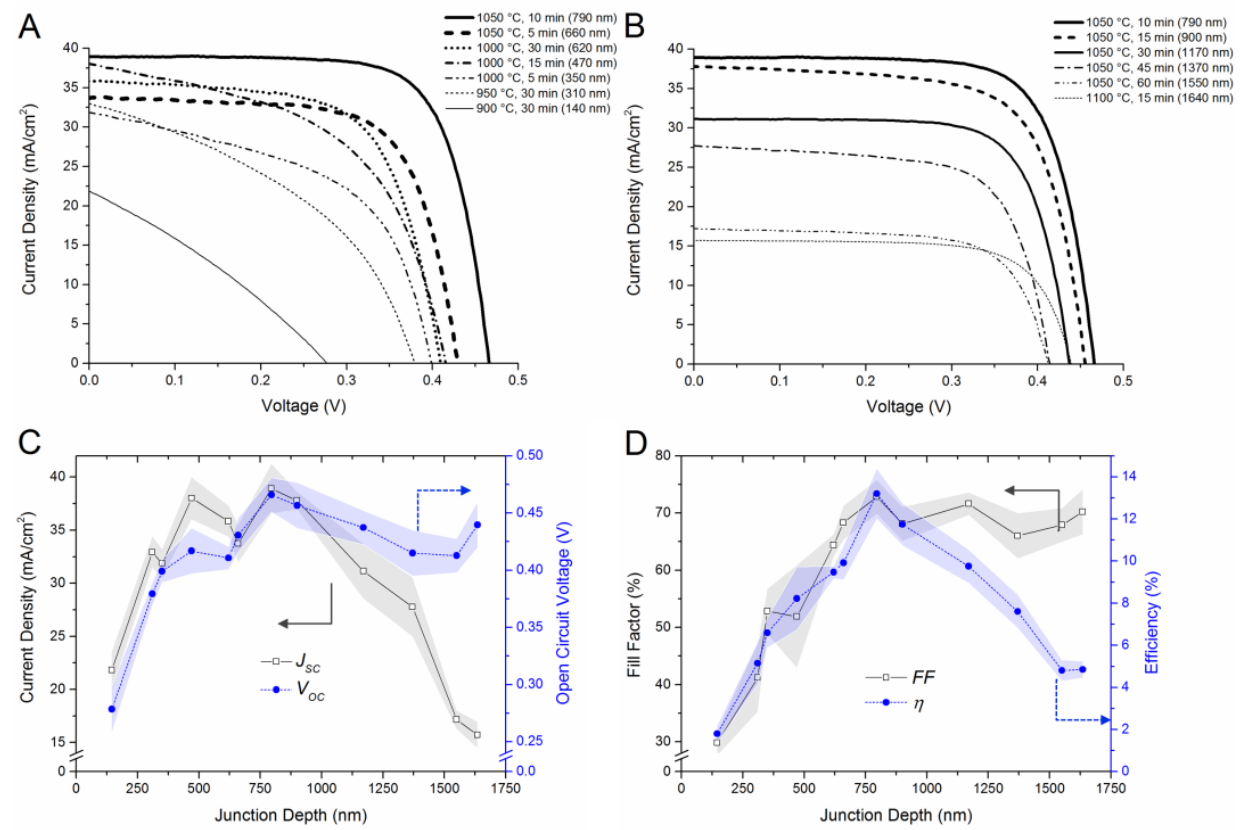

Figure 4.4: $J V$ measurements of various $n$-doped $p$-type base micropillar arrays for different junction depths, each line represents the average of at least 4 different samples of the same setting. (A) $J V$ data for junction depths between 140-660 nm. (B) $J V$ data for junction depths between $790-1640 \mathrm{~nm}$. (C) Current density and open circuit voltage as a function of the junction depth shown in $A$ and $B$, error bars indicate the 10-range. (D) Corresponding fill factor and efficiency of the average values shown in $\mathrm{C}$.

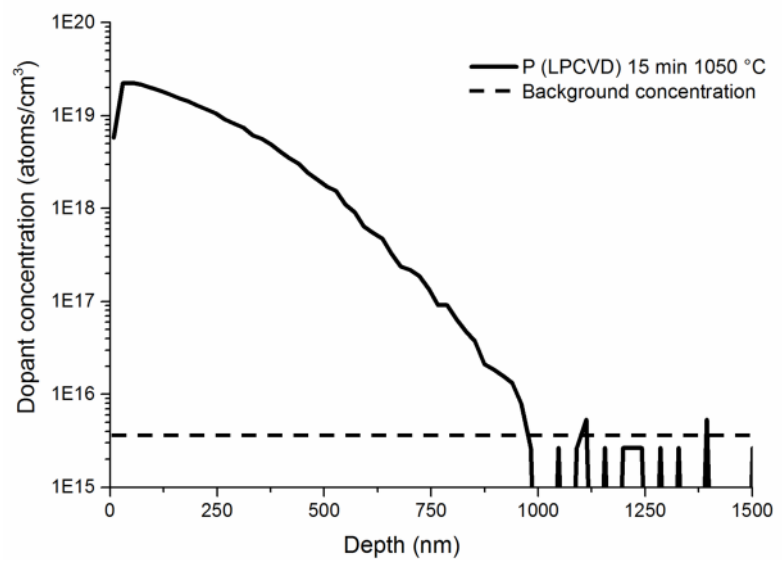

Figure 4.5: SIMS measurement of a flat sample, doped with LPCVD of phosphorus at $1050^{\circ} \mathrm{C}$, for $15 \mathrm{~min}$. The dotted line indicates the base boron concentration of the used wafer, prior to doping, estimated by a 4-point probe measurement. 
For junctions above $1.55 \mu \mathrm{m} J_{S C}$ dropped even below the current density of flat samples $\left(22 \mathrm{~mA} / \mathrm{cm}^{2}\right.$, see Figure 4.3A). Some insight in this behavior can be obtained by calculating the doping profile plus the depletion zone in the radially doped pillars. For $\mathrm{n}^{+} / \mathrm{p}$ junctions as used here, the donor density $\left(N_{D}\right)$ is much larger than the acceptor density $\left(N_{A}\right)$, therefore the depletion zone $W_{\text {dep }}$ can be approximated by: $:^{[27]}$

$$
W_{\text {dep }} \approx \sqrt{\frac{2 \varepsilon_{S i} \Phi_{b i}}{q N_{A}}}
$$

where $\varepsilon_{S i}$ is the permittivity of silicon, $\Phi_{b i}$ is the built-in potential $(\mathrm{V}), q$ is the electric charge and $N_{A}$ is the acceptor density $\left(\right.$ atoms $\left./ \mathrm{cm}^{3}\right)$. The built-in voltage is defined as: ${ }^{[27]}$

$$
\Phi_{b i}=\frac{k T}{q} \ln \frac{N_{D} N_{A}}{n_{i}^{2}}
$$

where $k$ is the Boltzmann constant, $T$ is the temperature $(\mathrm{K})$, and $n_{i}$ is the intrinsic carrier concentration $\left(\right.$ atoms $/ \mathrm{cm}^{3}$ ). Equation 4.2 gives a built-in potential of $0.67 \mathrm{~V}$ for a base doping level of $2 \times 10^{15}$ atoms $/ \mathrm{cm}^{3}$. $W_{\text {dep }}$ largely depends on $N_{A}$, and since the used substrates have a resistivity of $5-10 \Omega \mathrm{cm}$, the depletion zone was calculated to be in the range of 584 to $808 \mathrm{~nm}$. Because the doping level of the introduced $n$ dopants is much higher than the base $p$ doping, the depletion zone can be expected to be located mainly from the junction depth inward into the core of the pillar. This implies that $4 \mu \mathrm{m}$ pillars with junctions deeper than $1.2-1.4 \mu \mathrm{m}$ will have no p-type core left. This agrees favorably with the experimental data for junction depths of $1.37 \mu \mathrm{m}$ and higher (Figure 4.4B). Summarizing, our pillar arrays, in combination with LPCVD phosphorus doping, show an optimal $x_{j}$ value of approximately $800 \mathrm{~nm}$. Simulations have shown that thinner junctions should perform better. ${ }^{[18,20]}$ To make such thinner junctions experimentally with good diode quality, a doping method should be used which gives a higher dopant surface concentration. 


\subsection{Conclusions}

The solar cell performance of radially $n$-doped silicon pillar arrays was studied as a function of the pillar height and junction depth. Compared to flat samples, pillar arrays gave rise to a large increase in current density, from $22 \mathrm{~mA} / \mathrm{cm}^{2}$ for flat samples ( $6 \%$ efficiency) up to $37 \mathrm{~mA} / \mathrm{cm}^{2}$ for $40 \mu \mathrm{m}$ pillar arrays (12\% efficiency, $900 \mathrm{~nm}$ junction). However, for heights of 50 and $60 \mu \mathrm{m}, J_{S C}$ decreased, which is attributed to increased numbers of surface defect states, resulting from the deep reactive ion etching process. This effect was counteracted by an $\mathrm{Al}_{2} \mathrm{O}_{3}$ passivation layer. The LPCVD process for phosphorus doping did not give proper diodes for shallow radial junctions. The optimum junction depth found experimentally was $790 \mathrm{~nm}$ (13\% efficiency for $40 \mu \mathrm{m}$ high pillars). For deeper junctions, a steady decrease of $J_{S C}$ was observed, which is explained by the narrowing $\mathrm{p}$ core of the pillars, resulting in efficiencies poorer than flat silicon surfaces for junction depths exceeding approximately $1.2 \mu \mathrm{m}$. Further research towards more efficient silicon pillar array solar cells should focus on the fabrication of shallower junctions, $<600$ $\mathrm{nm}$, using a more effective doping technique, in combination with optimized passivation and anti-reflective coatings. In addition, future research should also verify what the optimal pillar configuration is (e.g. nano/micro-scale), combined with different $D / P$ ratios.

\section{Acknowledgements}

Wouter Vijselaar is acknowledged for the discussions about this chapter and for his assistance with the $J V$ measurements. Roald Tiggelaar is thanked for corrections and discussions on this chapter. 


\subsection{References}

[1] E. Garnett, P. Yang, Nano Lett., 2010, 10, 1082-1087.

[2] M.G. Walter, E.L. Warren, J.R. McKone, S.W. Boettcher, Q. Mi, E.A. Santori, N.S. Lewis, Chem. Rev., 2010, 110, 6446-6473.

[3] Y. Li, Q. Chen, D. He, J. Li, Nano Energy, 2014, 7, 10-24.

[4] J. Jin-Young, G. Zhongyi, J. Sang-Won, U. Han-Don, P. Kwang-Tae, H. Moon Seop, Y. Jun Mo, L. Jung-Ho, Nanotechnology, 2010, 21, 445303.

[5] B.M. Kayes, M.A. Filler, M.C. Putnam, M.D. Kelzenberg, N.S. Lewis, H.A. Atwater, Appl. Phys. Lett., 2007, 91, 103110.

[6] K. Peng, A. Lu, R. Zhang, S.-T. Lee, Adv. Funct. Mater., 2008, 18, 3026-3035.

[7] R. Elbersen, R.M. Tiggelaar, A. Milbrat, G. Mul, H. Gardeniers, J. Huskens, Adv. Energy Mater., 2015, 5, 1401745.

[8] B.M. Kayes, H.A. Atwater, N.S. Lewis, J. Appl. Phys., 2005, 97, 114302.

[9] H.P. Yoon, Y.A. Yuwen, C.E. Kendrick, G.D. Barber, N.J. Podraza, J.M. Redwing, T.E. Mallouk, C.R. Wronski, T.S. Mayer, Appl. Phys. Lett., 2010, 96, 213503.

[10] I. Oh, J. Kye, S. Hwang, Nano Lett., 2011, 12, 298-302.

[11] S.W. Boettcher, E.L. Warren, M.C. Putnam, E.A. Santori, D. Turner-Evans, M.D. Kelzenberg, M.G. Walter, J.R. McKone, B.S. Brunschwig, H.A. Atwater, N.S. Lewis, J. Am. Chem. Soc., 2011, 133, 1216-1219.

[12] Y. Hou, B.L. Abrams, P.C.K. Vesborg, M.E. Björketun, K. Herbst, L. Bech, A.M. Setti, C.D. Damsgaard, T. Pedersen, O. Hansen, J. Rossmeisl, S. Dahl, J.K. Nørskov, I. Chorkendorff, Nat. Mater., 2011, 10, 434-438.

[13] S.Y. Reece, J.A. Hamel, K. Sung, T.D. Jarvi, A.J. Esswein, J.J.H. Pijpers, D.G. Nocera, Science, 2011, 334, 645-648.

[14] E.L. Warren, H.A. Atwater, N.S. Lewis, J. Phys. Chem. C, 2013, 118, 747-759.

[15] M.D. Kelzenberg, S.W. Boettcher, J.A. Petykiewicz, D.B. Turner-Evans, M.C. Putnam, E.L. Warren, J.M. Spurgeon, R.M. Briggs, N.S. Lewis, H.A. Atwater, Nat. Mater., 2010, 9, 239-244.

[16] F. Voigt, T. Stelzner, S. Christiansen, Prog. Photovolt. Res. Appl., 2012, 21, 1567-1579.

[17] D. Zhou, Y. Pennec, B. Djafari-Rouhani, O. Cristini-Robbe, T. Xu, Y. Lambert, Y. Deblock, M. Faucher, D. Stiévenard, J. Appl. Phys., 2014, 115, 134304.

[18] F. Wang, H. Yu, J. Li, S. Wong, X.W. Sun, X. Wang, H. Zheng, J. Appl. Phys., 2011, 109, 114301.

[19] J. Li, H. Yu, S.M. Wong, G. Zhang, X. Sun, P.G.-Q. Lo, D.-L. Kwong, Appl. Phys. Lett., 2009, 95, 243113.

[20] A. Dalmau Mallorquí, F.M. Epple, D. Fan, O. Demichel, A. Fontcuberta i Morral, Phys. Status Solidi A, 2012, 209, 1588-1591.

[21] M. Uematsu, J. Appl. Phys., 1997, 82, 2228-2246.

[22] O. Gunawan, K. Wang, B. Fallahazad, Y. Zhang, E. Tutuc, S. Guha, Prog. Photovolt. Res. Appl., 2011, 19, 307-312.

[23] H. Xu, N. Lu, D. Qi, J. Hao, L. Gao, B. Zhang and L. Chi, Small, 2008, 4, 1972-1975.

[24] L.-B. Luo, X.-B. Yang, F.-X. Liang, H. Xu, Y. Zhao, X. Xie, W.-F. Zhang, S.-T. Lee, J. Phys. Chem. C, 2011, 115, 18453-18458.

[25] H. Jansen, M. de Boer, S. Unnikrishnan, M.C. Louwerse and M. Elwenspoek, J. Micromech. Microeng., 2009, 19, 033001.

[26] G. Dingemans, R. Seguin, P. Engelhart, M.C.M. van de Sanden, W.M.M. Kessels, Phys. Status Solidi RRL, 2010, 4, 10-12.

[27] C.C. Hu, Modern Semiconductor Devices for Integrated Circuits, Prentice Hall, United States, 2009. 


\section{Chapter 5}

\section{Electrical Characterization of Silicon Micropillars with Radial $\mathbf{p} / \mathbf{n}$ Junctions Containing Passivation and Anti- Reflection Coatings}

The effects of anti-reflective and passivation layers were investigated to optimize hexagonal packed silicon micropillar arrays, with radial $p / n$ junctions incorporated. These anti-reflection coatings $\left(\mathrm{Al}_{2} \mathrm{O}_{3}, \mathrm{SiO}_{2}, \mathrm{SiN}_{x}\right.$ and ITO) were first grown on flat solar cells with varying thickness, to verify the optimal thickness for light capturing calculated from theory by the Fresnel equations. $\mathrm{Al}_{2} \mathrm{O}_{3}, \mathrm{SiO}_{2}$, and $\mathrm{SiN}_{x}$ were chosen because of the possible two-fold improvement function, namely passivation and anti-reflection properties. ITO was chosen because of the three-fold function, passivation, anti-reflection and conductive properties. These layers were grown on both $n$-type base ( $p$-emitter) and $p$-type base ( $n$-emitter) silicon pillar substrates with radial $p / n$ junctions, using different methods. Upon analysis of these structures using high-resolution scanning electron microscopy, atomic layer deposition of $\mathrm{Al}_{2} \mathrm{O}_{3}$ gave the best uniformity over the whole pillar range, compared to a low-pressure chemical vapor deposition of $\mathrm{SiO}_{2}, \mathrm{SiN}_{x}$, and sputtering of ITO. For the latter three materials coatings, the layers were not homogeneously distributed, but this was of no influence on the properties of the coatings. For ITO, a loss of indium atoms was visible by HR-SEM. In terms of electrical characterization, all samples, with the exception of ITO, outperformed the flat silicon solar cell. The $\mathrm{Al}_{2} \mathrm{O}_{3}$ samples performed best, for both $p$ - and n-type base pillars, increasing the efficiency from 6.1 to $13.4 \%$ ( $p$-type) and 7.4 to $10.1 \%$ (n-type). 


\subsection{Introduction}

Silicon has been used as a materials in photovoltaic (PV) cells, and have been applied intensively for many decades. ${ }^{[1-3]}$ Silicon is an earth abundant, stable and non-toxic semiconductor, in addition, the knowledge gained in the fields of nano/micro-engineering of silicon enabled the fabrication of complex silicon solar cells. In particular, the development from 2D to 3D structures has enabled the fabrication of nano/micropillar structures with radial junctions ${ }^{[4-6]}$ and their use in PV and solar-to-fuel devices. ${ }^{[7-9]}$ These nano/micropillars have gained increasing attention, owing to the advantages of an increase in junction area for a given footprint, as well as their ability to orthogonally decouple the directions of incoming light and charge separation. ${ }^{[10,11]}$ Moreover, micropillars (and other microstructures such as pyramids, trenches, and surface roughening) decrease the reflectivity of a silicon surface and thus increase the amount of charges that can be theoretically collected from a given footprint or surface area. ${ }^{[4,12,13]}$ On the other hand, the efficiency of a solar cell is determined, among other variables, by the amount of collected charges, which is lowered by the extent of recombination in the solar cell and by the reflected light. Especially for high aspect ratio $3 \mathrm{D}$ nano/microstructures, surface recombination is an important factor, due to the increased surface area.

Surface recombination can be reduced by surface passivation. Surface passivation methods can be subdivided into two categories: chemical and field-effect passivation. ${ }^{[14,15]}$ Recombination consumes both an electron and a hole, therefore if either one is trapped, the probability for recombination increases significantly. In reality, the energy trap states are associated with surface defects (for example, dangling bonds) and are distributed throughout the band gap due to slight variations in structure and bond angle. These surface defects can be reduced by the application of a surface coating, which method is referred to as chemical passivation, which effectively reduces the recombination rate. ${ }^{[16]}$ Another way to inhibit recombination is by a significant 
reduction in the density of the minority charge carriers at the surface by an electric field. An electric field can be induced by means of the introduction of fixed charges at the surface of the crystalline silicon (c-Si). This is called field-effect passivation. ${ }^{[17,18]}$

Surface coatings have multiple influences on the performance of a silicon solar cell, foremost by their role in surface passivation. ${ }^{[19,20]}$ Already a relatively thin layer, of a few nanometers, is sufficient to obtain either or both chemical and field-effect passivation. If the thickness of the passivation layer is increased, the coating can also function as an anti-reflective coating (AR coating), provided that it has the correct thickness (i.e. tens of nanometers), a low extinction coefficient, and a refractive index that is lower than that of silicon. ${ }^{[21,22]}$ Aluminum oxide $\left(\mathrm{Al}_{2} \mathrm{O}_{3}\right)$, silicon-rich silicon nitride $\left(\mathrm{SiN}_{\mathrm{x}}\right)$, and silicon dioxide $\left(\mathrm{SiO}_{2}\right)$ are often used, non-conducting surface coatings. An electrically conductive and optically transparent coating can improve the efficiency even further, by enhancing the charge collection at the emitter, thereby effectively reducing the distance that charges have to travel through the emitter. Such a coating can thus fulfill a three-fold function: as a passivation layer, as an AR coating, and in the reduction of the resistance in the cell. Transparent conductive oxides are such triple-function coatings, and indium tin oxide (ITO) is the most widely applied. ${ }^{[23-25]}$

Here, we show the realization and characterization of various surface coatings on micropillar arrays with a radial $\mathrm{p} / \mathrm{n}$ junction, to function as a passivation and AR layer, in order to increase the overall efficiency of PV cells. In order to function as AR coating, different optimized thicknesses are required on $p$ - and n-type silicon solar cells. To find the optimal thickness, the performance of each coating has been simulated by means of finite element modeling, in order to predict the reflectivity of silicon upon the structuring with arrays of micropillars and applying an AR coating. For the deposition of non-conductive coatings, two different techniques have been used: atomic layer deposition (ALD) of $\mathrm{Al}_{2} \mathrm{O}_{3}$ and low-pressure chemical vapor deposition (LPCVD) of $\mathrm{SiN}_{\mathrm{x}}$ and $\mathrm{SiO}_{2}$. In addition, we have investigated the conformality of these layers, by 
analyzing the thickness on the sidewalls of the silicon micropillars, and evaluated the electrical characteristics of the coated solar cells. The 3D thickness analysis has been achieved by the combination of focused ion beam (FIB) etching and high resolution scanning electron microscopy (HR-SEM).

\subsection{Materials and methods}

\subsubsection{Finite Element Simulations of the reflectivity of silicon micro pillars}

The reflectivity of cubic packed micropillar arrays was simulated using a finite element modeling package (COMSOL Multiphysics 4.4). The simulation was performed by a parametric sweep (20 nm step) in the wavelength domain of visible light $(400-1100 \mathrm{~nm})$. The pitch and diameter of the micropillars were set to 6 and $4 \mu \mathrm{m}$ respectively, and the height of the micropillars was varied between 0 and $40 \mu \mathrm{m}$ (similar to the experimentally realized pillar arrays). The slab of silicon underneath the micropillar array was limited to $1 \mu \mathrm{m}$. The footprint of the fabricated arrays was $5 \times 5 \mathrm{~mm}^{2}$, which is orders of magnitude larger than the wavelength of the incoming light, therefore we only simulated one pillar of which the outcome was periodically repeated in the lateral directions. For this reason, the side boundaries of the simulation had a Floquet periodicity. The incoming light was a plane wave in the z-direction and had an input power of $1 \mathrm{~W}$. The maximum free triangular mesh size in the used materials (silicon and air) was set to $1 / 5$ of the minimum wavelength in the material. Furthermore, the opposing boundaries were symmetrical with respect to each other to ensure the Floquet periodicity. The realized and simulated pillar geometries are depicted below (Figure 5.1). 

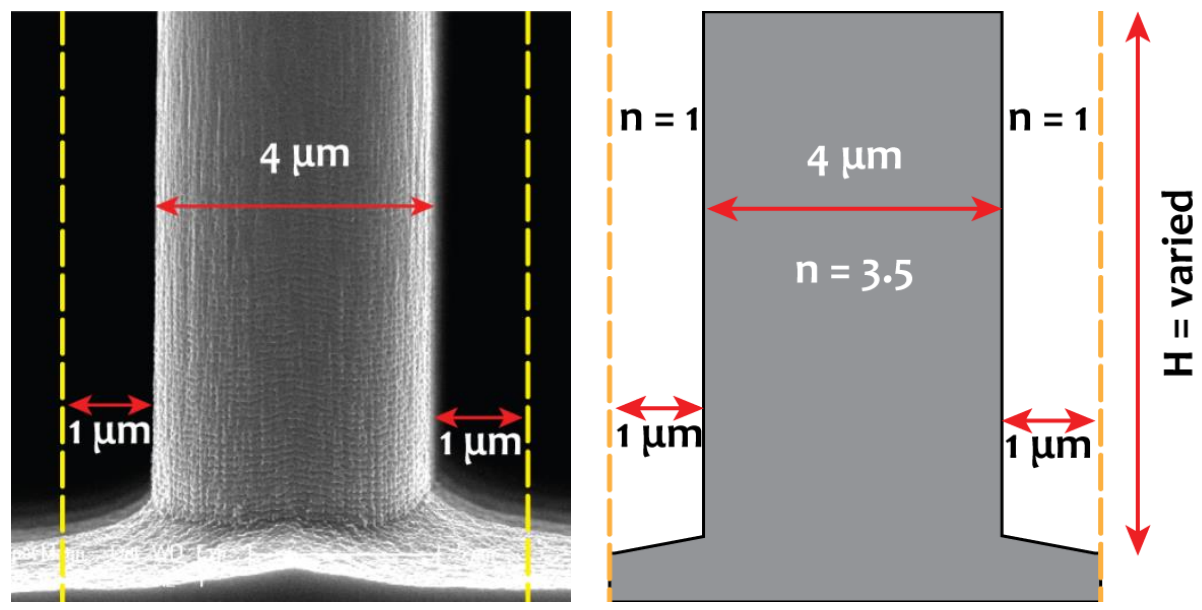

Figure 5.1: The real and simulated geometry of the COMSOL simulation. Note the curved bottom of the pillar.

\subsubsection{Fabrication of radial $\mathrm{p} / \mathrm{n}$ junctions in silicon micropillar arrays}

Figure 5.2 gives a schematic representation of the fabrication and coating of an array of micropillars with radial $\mathrm{p} / \mathrm{n}$ junctions. $\mathrm{P}$ - and $\mathrm{n}$-type silicon (100) substrates, with resistivities of 5-10 $\Omega \mathrm{cm}$ for p-type and 1-10 $\Omega \mathrm{cm}$ for n-type, $100 \mathrm{~mm}$ diameter, $525 \mu \mathrm{m}$ (p-type) and $375 \mu \mathrm{m}$ (n-type) thickness, single side polished (Okmetic Finland), were prepared as previously reported. ${ }^{[26]}$ In short, substrates were cleaned and covered with $100 \mathrm{~nm}$ silicon-rich silicon nitride $\left(\mathrm{SiN}_{\mathrm{x}}\right)$, via LPCVD. The silicon nitride on the front side of the wafer was removed by reactive ion etching (RIE, Adixen AMS100DE), and cleaned by means of oxygen plasma (30 min) and piranha (mixture of sulfuric acid and $30 \%$ aqueous hydrogen peroxide, 3:1 (v/v), $20 \mathrm{~min}$ ). By means of standard photolithography, squares $\left(5 \times 5 \mathrm{~mm}^{2}\right)$ with hexagonally packed circles (4 $\mu \mathrm{m}$ diameter, $2 \mu \mathrm{m}$ spacing) were defined in a photoresist polymer (Olin 906-12). Silicon micropillars (40 $\mu \mathrm{m}$ in height) were etched into the silicon substrate by deep reactive ion etching (DRIE, Bosch process) and the substrates were cleaned subsequently in oxygen plasma (30 $\mathrm{min}$ ) and piranha (20 $\mathrm{min}$ ) to remove the photoresist and fluorocarbon residues. $\mathrm{P} / \mathrm{n}$ and $\mathrm{n} / \mathrm{p}$ junctions were formed by the deposition of boron oxide (using solid source dotation, SSD) and phosphorus oxide (LPCVD), respectively. 
For SSD of boron, boron nitride wafers were placed in between the silicon target wafers and, under a flow of $6000 \mathrm{sccm} \mathrm{O}_{2}$, a boron oxide layer was formed on the silicon surface. In case of the LPCVD of phosphorus, a mixture of $330 \mathrm{sccm} \mathrm{PH}_{3}$ and $50 \mathrm{sccm} \mathrm{O}_{2}$ was supplied for $45 \mathrm{~min}$ at $350 \mathrm{mTorr}$ in order to deposit the phosphorus oxide on the silicon surface. The formed dopant oxide layers were then annealed at $1050{ }^{\circ} \mathrm{C}$ for $15 \mathrm{~min}$ to accomplish diffusion of the dopant into the silicon micropillars, to form the proper radial junction. Prior to the deposition of the passivation layers, the dopant oxide was stripped in buffered hydrogen fluoride solution (BHF, $10 \mathrm{~min}$ ), upon which the wafers returned to their hydrophobic state. Subsequently, the wafers were cleaned by immersion in $100 \%$ nitric acid $\left(\mathrm{HNO}_{3}, 2 \times 5 \mathrm{~min}\right)$, fuming $69 \%$ nitric acid (10 min) and immersion in 1\% aqueous hydrofluoric acid (HF) to remove the native oxide ( $1 \mathrm{~min}$ ). The passivation layers were applied using different methods as described in detail in sections 5.2.3-5.2.5. In short, sputtering was chosen as a control, because the directionality of this deposition technique is expected to lead to a non-conformal coating of high aspect ratio microstructures, which then can be compared with the highly conformal methods of LPCVD and ALD. Ohmic contacts were realized by selectively removing the surface coating around the pillar arrays by completely filling and covering the pillar arrays with photoresist. HMDS was spin-coated in between the pillar arrays (4000 rpm, $2 \mathrm{~min}$ ), and subsequently AZ9260 photoresist was spin-coated on the samples (1000 rpm, $4 \mathrm{~min}$ ). The samples were dried overnight at $10^{-3} \mathrm{mbar}$ at room temperature. Hereafter, the samples were exposed to photolithography ( 3 times $10 \mathrm{~s}$ UV, $10 \mathrm{~s}$ delay) and developed (8 $\mathrm{min}$ ). $\mathrm{Al}_{2} \mathrm{O}_{3}$ and $\mathrm{SiO}_{2}$ were both stripped by wet etching in $\mathrm{BHF}$ (etch rates: $180 \mathrm{~nm} / \mathrm{min}$ and $210 \mathrm{~nm} / \mathrm{min}$, respectively), $\mathrm{SiN}_{\mathrm{x}}$ was dry etched by RIE (Adixen AMS100DE). The last step was removal of the photoresist mask layer and fluorocarbon residues using oxygen plasma (Tepla360). An Ohmic contact ( $1 \mu \mathrm{m}, \mathrm{Al} / \mathrm{Si}, 99 / 1 \%$ ) was sputtered (Oxford PL400) onto the backside of the wafer and onto the front side, the latter by means of a shadow mask to prevent deposition on the pillars arrays. 


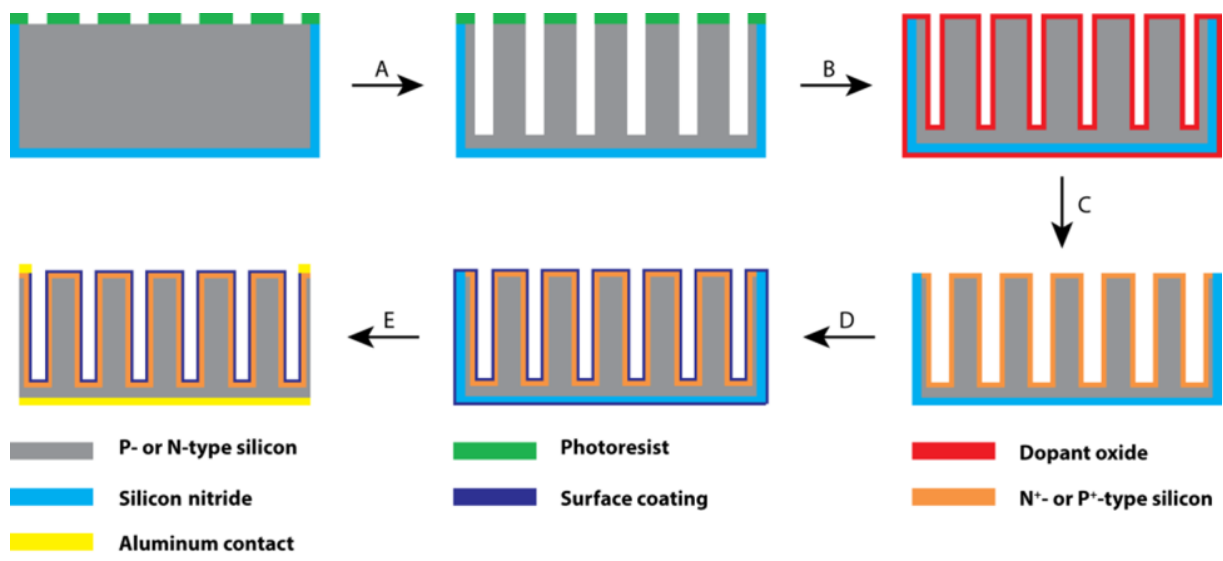

Figure 5.2: Schematic fabrication process. (A) Deep reactive ion etching of a silicon wafer with a photoresist pattern on the front side and silicon-rich silicon nitride on the backside. (B) Deposition of the dopant oxide by either SSD (boron) or LPCVD (phosphorus). (C) Formation of the radial junction, by a drive-in step at $1050^{\circ} \mathrm{C}$ for $15 \mathrm{~min}$. (D). Deposition of a passivation layer by either $\operatorname{ALD}\left(\mathrm{Al}_{2} \mathrm{O}_{3}\right)$, $\operatorname{LPCVD}\left(\mathrm{SiO}_{2}, \mathrm{SiN}_{\mathrm{x}}\right)$ or sputtering (ITO). (E) Removal of the silicon nitride and deposition of aluminum front and back contact by sputtering.

\subsubsection{Atomic layer deposition}

To reduce the reflectivity and to passivate the micropillar arrays, a layer of aluminum oxide was conformally coated onto the pillar arrays. $\mathrm{Al}_{2} \mathrm{O}_{3}$ films were grown in a Picosun P-300S reactor by a thermal ALD process at $100{ }^{\circ} \mathrm{C}$. Trimethyl-aluminum (TMA) and $\mathrm{H}_{2} \mathrm{O}$ were used as precursors and $\mathrm{N}_{2}$ was the carrier gas, yielding a growth rate of $0.83 \AA$ per cycle (after initial growth stabilization). The time for one complete $\mathrm{Al}_{2} \mathrm{O}_{3}$ ALD cycle was $9.3 \mathrm{~s}$, where each cycle was defined as: $0.1 \mathrm{~s}$ TMA exposure, $4 \mathrm{~s} \mathrm{~N}_{2}$ purge, $0.2 \mathrm{~s} \mathrm{H}_{2} \mathrm{O}$ exposure, and $5 \mathrm{~s}_{2}$ purge.

\subsubsection{Low-pressure chemical vapor deposition}

The deposition of silicon oxide and silicon nitride films was done by LPCVD. $\mathrm{SiO}_{2}$ was grown at $725^{\circ} \mathrm{C}$ with a $50 \mathrm{sccm}$ tetraethyl orthosilicate flow (200 mTorr, $8.1 \mathrm{~nm} / \mathrm{min}$ deposition rate). For $\mathrm{SiN}_{\mathrm{x}}$, a flow of $50 \mathrm{sccm} \mathrm{SiH}_{2} \mathrm{Cl}_{2}$ and $150 \mathrm{sccm} \mathrm{NH}_{3}$ was used at a pressure of 250 mTorr at $825^{\circ} \mathrm{C}$ (6.6 $\mathrm{nm} / \mathrm{min}$ deposition rate). 


\subsubsection{Sputtering}

Indium tin oxide (ITO) was deposited by means of an in-house built sputter device. The silicon target wafer was placed on a rotating chuck (5 rpm), $44 \mathrm{~mm}$ from the ITO source, in a vacuum chamber $\left(5.5 \times 10^{-3} \mathrm{mbar}\right)$ with a $40 \mathrm{sccm}$ flow of argon in the reactor chamber. The ITO source had an angle of $45^{\circ}$ with respect to the wafer surface. By means of a DC power of $50 \mathrm{~W}$ and a $20 \mathrm{sccm}$ argon flow, ITO was sputtered on the silicon substrate $(2.6 \mathrm{~nm} / \mathrm{min})$.

\subsubsection{Focused ion beam}

FIB structures were made with a Nova 600 Dual Beam - SEM/FIB setup. A $\mathrm{Ga}^{+}$liquid metal ion source was used to mill away approximately half of a single pillar in the z-direction with respect to the footprint of an array, with a beam current of $0.92 \mathrm{nA}$ and a $10 \mathrm{kV}$ extraction voltage.

\subsection{7. $J V$ measurements}

To measure the electrical characteristics of the radial $p / n$ junctions, samples were positioned perpendicular to a $300 \mathrm{~W}$ xenon arc light source, with an intensity of one sun (AMG 1.5). JV measurements were recorded on a VersaSTAT 4 potentiostat using a linear voltage sweep from -0.7 to $0.7 \mathrm{~V}$ at a rate of $0.2 \mathrm{~V} / \mathrm{s}$. For each variation in sample (i.e. type of coating and/or layer thickness), at least four specimens were measured. Averages are given in each figure and standard deviations are listed in the accompanying table.

\subsection{Results and discussion}

\subsubsection{Simulations}

Infinite flat silicon substrates reflect $35 \%$ of the incoming light. This can be analytically calculated by the Fresnel equations (Figure 5.3, analytical model). In order to enhance the overall efficiency of a PV cell, reduction of reflection of is of crucial importance. This is done by either an anti-reflection coating, structuring the surface or a combination of both. In order to apply a correct coating thickness on the surface to maximize the solar cells electrical output, 
the reflection of the structured surface as function of wavelength is needed. FEM is a powerful tool to simulate structures over a broad wavelength spectrum. In order to confirm the FEM simulation, it is compared to the analytical Fresnel model. As can be seen in Figure 5.3A, there is fairly good agreement between the analytical Fresnel model and the FEM simulation. The periodical ripples are due to simulating only a slab of silicon with a thickness of $1 \mu \mathrm{m}$. This introduces a double air-silicon interface (front- and backside) and gives rise to an interference pattern, known as the Fabry-Perot effect. The analytical model assumes an infinite thick slab and has only one silicon/air interface, ergo, no interference pattern is visible. The reflectivity is lowered substantially upon the addition of pillars with a height of $5 \mu \mathrm{m}$ (Figure 5.3A) and lowered even further by increasing the height of the pillars up to $40 \mu \mathrm{m}$. Based on the FEM simulations, we postulate that light absorption for this hexagonal packing of the pillar array with a height of $40 \mu \mathrm{m}$ is limited by the packing density of the pillar arrays within the footprint. Since the diameter of the pillars is an order of magnitude larger than the wavelength of the incoming light, the top surfaces of the pillars will reflect light as a flat surface. Light entering the space in between the pillars will be scattered multiple times to such an extent that it will be absorbed by the pillars. The top surfaces of the pillars add up to $34 \%$ of the total array area, therefore $66 \%$ of the light is assumed to be completely absorbed and $34 \%$ reflected as if it was on a continuous surface of polished silicon. Figure 5.3A also includes a curve of $34 \%$ of the theoretical reflection of a flat surface. This curve is in reasonable agreement with the simulation done for the $40 \mu \mathrm{m}$ high pillars. 

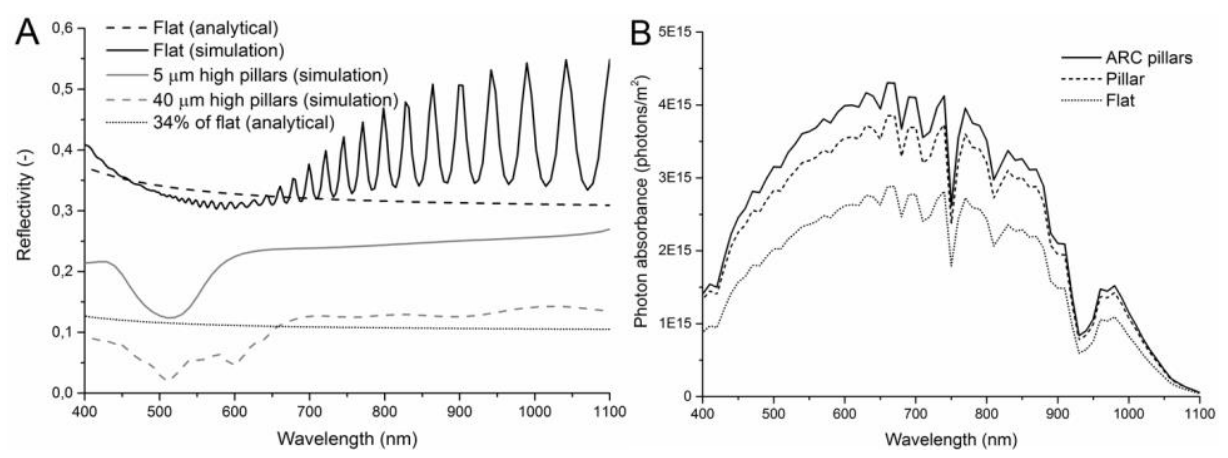

Figure 5.3: $(A)$ The simulation results of the reflection of pillars with different heights. The results include the theoretical reflection of a flat silicon surface and when only $34 \%$ of the light is reflected of a flat surface. (B): The theoretical photon absorbance of flat, pillar array and coated pillar array.

With the reflection spectrum known, the optimal thickness of the AR coating was calculated by considering a combination of three spectra as a function of the wavelength, namely the photon flux ${ }^{[27]}$ the internal quantum efficiency $(\mathrm{IQE}),{ }^{[28]}$ and the reflectivity of the sample. By multiplying these spectra, the theoretical photon absorbance of the silicon cell was obtained (Figure 5.3B). The surface area underneath each curve in Figure 5.3B is directly reflected in the current density output of a complete solar cell. Therefore, the photon absorption capabilities of a flat surface are increased by adding an anti-reflection coating to the surface, and are even more improved by applying an AR coating to these pillars (see Figure 5.3B). The thickness of an AR coating is such, that it has a minimum in reflection at the maximum photon flux in the visible light region $(\sim 600 \mathrm{~nm})$. To be specifically for the latter, the total amount of photons can be maximized by adjusting the minimum in the reflectivity curve. The minimum in the reflectivity is directly coupled to the thickness of the AR coating and the refractive index of the used coating. The optimized calculated thickness values in current densities are given in Table 5.1 for three anti-reflection coatings.

\subsubsection{Anti-reflection coatings}

Different AR coatings $\left(\mathrm{Al}_{2} \mathrm{O}_{3}, \mathrm{SiO}_{2}, \mathrm{SiN}_{\mathrm{x}}\right)$ were deposited on flat ( $\mathrm{p}$-type) and pillared ( $p$ - and $n$-type) samples. Table 5.1 shows the ellipsometrically 
measured optimal thicknesses of the experimentally deposited AR coatings on both $\mathrm{p}$ - and n-type base pillar arrays with their corresponding doping.

Table 5.1: Simulated and measured optimal coating thicknesses of various AR coating materials.

\begin{tabular}{cccc}
\hline Material & $\begin{array}{c}\text { Optimal thickness } \\
(\mathbf{n m})^{\mathbf{a}}\end{array}$ & $\begin{array}{c}\text { Thickness on } \\
\text { p-type }(\mathbf{n m})^{\mathbf{b}}\end{array}$ & $\begin{array}{c}\text { Thickness on } \\
\mathbf{n} \text {-type }(\mathbf{n m})\end{array}$ \\
\hline $\mathbf{A l}_{2} \mathbf{O}_{3}$ & 91 & 87 & 86 \\
$\mathrm{SiN}_{\mathbf{x}}$ & 71 & 66 & 65 \\
$\mathrm{SiO}_{2}$ & 106 & 110 & 106 \\
\hline $\begin{array}{l}\text { a } \\
\text { Theoretically calculated }\end{array}$ & & & \\
Measured by ellipsometry & &
\end{tabular}

To inspect the coating over the entire height of the pillar and thus to assess the conformality of the AR coating layers, the coated pillars were cut in half along the z-direction, by means of FIB etching. An overview of pillars coated with $\mathrm{Al}_{2} \mathrm{O}_{3}, \mathrm{SiO}_{2}, \mathrm{SiN}_{\mathrm{x}}$ and ITO is given in Figure 5.4. Here, it is clearly visible that the deposition of $\mathrm{Al}_{2} \mathrm{O}_{3}$ by $\mathrm{ALD}$ has resulted in a perfectly conformal coating over the whole height of the pillar. In contrast, both LPCVD materials (i.e. $\mathrm{SiO}_{2}$ and $\mathrm{SiN}_{\mathrm{x}}$ ) exhibit a gradient in thickness along the height of the micropillar. At the bottom, the layer is almost twice as thick as at the top. Sputtering of ITO caused a problem in the scallops from the fabrication process of the micropillars (DRIE; Bosch process, extra highlighted in Figure 3). Due to the relatively high directionality of the material flux of physical vapor deposition (i.e. sputtering) as compared to ALD or LPCVD. This resulted in a non-conformal ITO thickness at the sidewalls of the pillars, at least at the top where scallops, resulting from the fabrication, cause a shadowing effect in the directional sputtering process. Further down the height of the pillar less scalloping is visible and here the pillar is continuously coated. For all coatings, the difference in conformality between the depositions of these materials is attributed to the different deposition methods used, although some removal of $\mathrm{SiO}_{2}$ and $\mathrm{SiN}_{\mathrm{x}}$ during $\mathrm{FIB}$ etching, which would lead to preferential removal from the tops of the pillar because of the concomitantly higher exposure times upon etching of the pillar, cannot be excluded. 


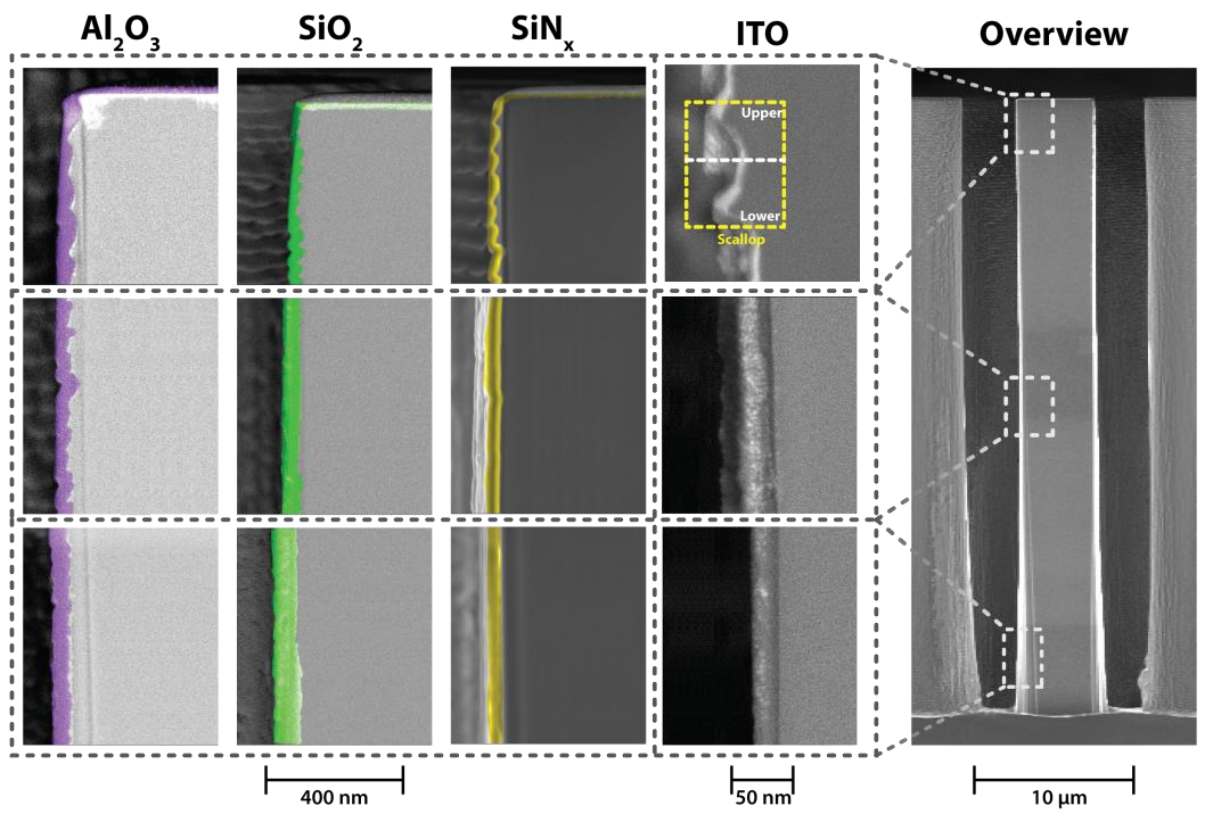

Figure 5.4: Cross-section HR-SEM images showing the layers of $\mathrm{Al}_{2} \mathrm{O}_{3}, \mathrm{SiN}_{\mathrm{x}}, \mathrm{SiO}_{2}$ and ITO along the sides of pillars that have been cut in half-orthogonal to the substrate to inspect the conformality of the AR coatings across the entire pillar height. The HR-SEM images are false colored manually in Photoshop to aid the visualization of the coatings. The pillar on the right has been coated by $\operatorname{ALD}\left(\mathrm{Al}_{2} \mathrm{O}_{3}\right)$.

The indium doping level of is utmost importance for ITO, since it is a conductive oxide because of the incorporation of indium atoms. One way to check and visualize the doping level of the coating over the height of the pillar, is by energy selective backscattered (ESB) SEM imaging. ESB is suitable for visualizing both the contrast between the applied coating and pillar as well as that between elements of which the layer is composed. From top to bottom, the indium content in the coating is reduced, as the ESB image at positions further down the pillar only shows some bright spots in the coating (see Figure 5.4, ITO). At the bottom, almost no indium is present and hence the coating is composed mostly of tin oxide. When indium is depleted from the layer, it is assumed that ITO loses its conductive property. 


\subsubsection{JV measurements}

To verify the positive effect of the deposited layers, $J V$ measurements were performed on flat surfaces and on both coated and non-coated silicon pillar arrays with radial junctions. Prior to the measurements of the unmodified flat and bare pillar arrays, the samples were dipped in $1 \% \mathrm{HF}(1 \mathrm{~min})$ to ensure removal of the native oxide.

Measurements on different thicknesses for pillared $p / n$ junctions were only performed on $\mathrm{p}$-type silicon (n-emitter). To verify whether the estimated optimal thickness (as shown in the simulations described above) was correct, $J V$ measurements were performed, and the current density output compared (Figure 5.5). This was done for $\mathrm{Al}_{2} \mathrm{O}_{3}, \mathrm{SiO}_{2}$ and $\mathrm{SiN}_{\mathrm{x}}$, and showed optimal thicknesses of 90,110 and $71 \mathrm{~nm}$ respectively, well in agreement with the simulated optimal values $(91,106,71 \mathrm{~nm}$, respectively).

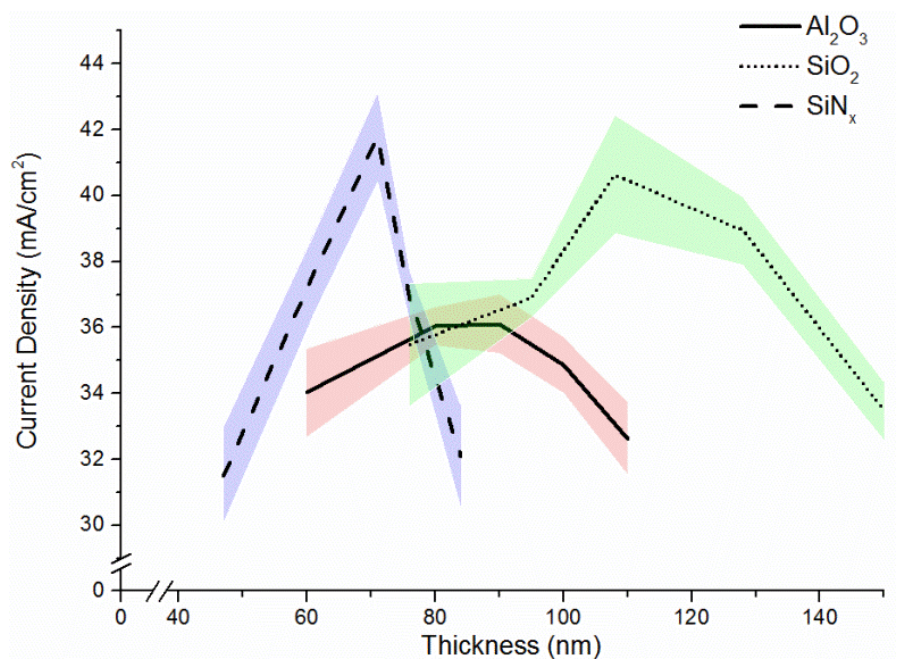

Figure 5.5: The current density output as a function of the thickness of the anti-reflection coating for $p$-base samples for $\mathrm{Al}_{2} \mathrm{O}_{3}, \mathrm{SiO}_{2}$ and $\mathrm{SiN}_{\mathrm{x}}$, doped at $1050{ }^{\circ} \mathrm{C}$ for 15 minutes. The transparent layers indicate the $1 \sigma$ error range.

All coated samples (Figure 5.6) show an improvement in current density compared to the bare pillar arrays, indicating that the layers are indeed functioning as passivation and/or AR coating, except for the ITO sample on $n$-type silicon. For the p-type base samples (n-emitter, Figure 5.6A), all 
samples show an improvement over the bare pillared samples, where $\mathrm{SiO}_{2}$ and $\mathrm{SiN}_{\mathrm{x}}$ show a larger improvement than $\mathrm{Al}_{2} \mathrm{O}_{3}$ in terms of $J_{S C}$ (Table 5.2). We contribute these improvements to a combination of effects. Firstly, all coatings have a refractive index different from silicon, and therefore each material will act as an anti-reflection coating. Secondly, all applied coatings passivate the silicon surface by reducing the amount of surface trap states by chemical passivation (i.e. reducing the amount of dangling silicon bonds, which is proportional to the total surface area of the micropillar array). Thirdly, from literature it is known that $\mathrm{SiO}_{2}$ and $\mathrm{SiN}_{\mathrm{x}}$ introduce a positively charged interface ${ }^{[16]}$ due to which the minority carriers (holes) in the n-emitter are electrically shielded from the surface and thus from the trap states (i.e. field effect passivation). ${ }^{[2]}$ In contrast, $\mathrm{Al}_{2} \mathrm{O}_{3}$ induces a negatively charged interface ${ }^{[16]}$ due to which the minority carriers (holes) are attracted towards the surface and thus into the possible trap states. This effectively increases the minority carrier concentration at the $\mathrm{C}-\mathrm{Si}$ interface, which limits the improvements of $\mathrm{J}_{s c}$ upon use of $\mathrm{Al}_{2} \mathrm{O}_{3}$. The reason for the large increase of $V_{O C}$ for the $\mathrm{Al}_{2} \mathrm{O}_{3}$ sample is unknown, however, we speculate that the chemical passivation of the ALD technique is more effective than that of the LPCVD methods. In terms of efficiency, all coatings lead to a significant increase compared to bare arrays, up to $13.4 \%$ for $\mathrm{Al}_{2} \mathrm{O}_{3}$, compared to the $6.1 \%$ of the bare pillars (see Table 5.2).

In case of the $n$-type base samples (p-emitter, Figure 5.6B), an opposite effect is observed. The increase in $J_{S C}$ is the highest for $\mathrm{Al}_{2} \mathrm{O}_{3}$, compared to the $\mathrm{SiO}_{2}$ and $\mathrm{SiN}_{\mathrm{x}}$ layers, which is expected based on the negatively charged $\mathrm{Al}_{2} \mathrm{O}_{3}$ interface with $\mathrm{c}-\mathrm{Si}$, whereas ITO performs slightly worse than the flat sample. The adverse effect of the positively charged interface upon coating with $\mathrm{SiO}_{2}$ or $\mathrm{SiN}_{\mathrm{x}}$ has the most impact on samples with $\mathrm{SiO}_{2}$ as passivation coating, since it is known that for $\mathrm{SiN}_{\mathrm{x}}$ field effect passivation, in this case negative, can be reduced by annealing which gives a reduction of the charge density of the overall positively charged interface. ${ }^{[30]} \mathrm{SiN}_{\mathrm{x}}$ can be deposited by plasma-enhanced chemical vapor deposition (PECVD) (at ca $400^{\circ} \mathrm{C}$ ) which is 
followed by an annealing step at $700{ }^{\circ} \mathrm{C}$. However, LPCVD $\mathrm{SiN}_{\mathrm{x}}$ is deposited at $825{ }^{\circ} \mathrm{C}$ and the annealing step is done at the same time. Similar to the p-type base samples, $\mathrm{Al}_{2} \mathrm{O}_{3}, \mathrm{SiO}_{2}$ and $\mathrm{SiN}_{\mathrm{x}}$ result in an increase in the overall efficiency compared to bare micropillars (up to $10.1 \%$ for $\mathrm{Al}_{2} \mathrm{O}_{3}$ ). The ITO layer shows an increase in current density, but the $V_{O C}$ and $F F$ are lower, resulting in a similar efficiency as its bare pillared counterpart.
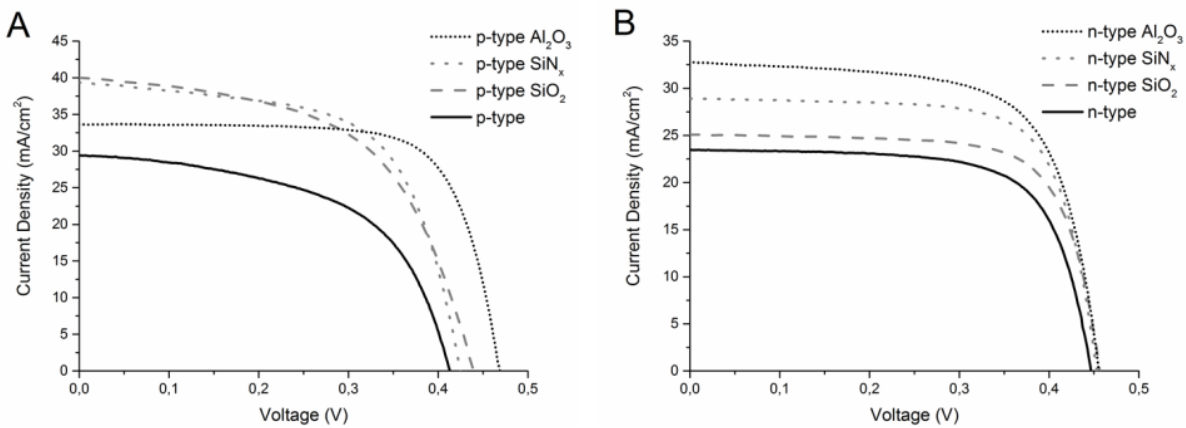

Figure 5.6: $J V$ measurements on $\mathrm{p}-(\mathrm{A})$ and $\mathrm{n}$-type (B) base micropillars with radial $\mathrm{p} / \mathrm{n}$ junctions, with and without coating materials.

Table 5.2: $J V$ characteristics for various coatings on both $\mathrm{p}$ - and n-type base specimens with $40 \mu \mathrm{m}$ silicon micropillars arrays.

\begin{tabular}{lcccc}
\hline \multicolumn{1}{|c}{ Sample } & $\begin{array}{c}\eta \\
(\%)\end{array}$ & $\begin{array}{c}J_{s c} \\
\left(\mathbf{m A} / \mathbf{c m}^{2}\right)\end{array}$ & $\begin{array}{c}V_{o c} \\
(\mathrm{~V})\end{array}$ & $\begin{array}{c}F F \\
(\%)\end{array}$ \\
\hline P-base bare & $6.1 \pm 1.3$ & $29.9 \pm 3.0$ & $0.41 \pm 0.01$ & $54 \pm 0.07$ \\
\hline P-base $\mathrm{Al}_{2} \mathrm{O}_{3}$ & $13.4 \pm 1.0$ & $33.6 \pm 2.7$ & $0.47 \pm 0.01$ & $73 \pm 0.01$ \\
\hline P-base $\mathrm{SiN}_{\mathbf{x}}$ & $10.4 \pm 1.4$ & $39.4 \pm 3.4$ & $0.42 \pm 0.02$ & $62 \pm 0.08$ \\
\hline P-base $\mathrm{SiO}_{2}$ & $10.2 \pm 1.2$ & $40.9 \pm 1.5$ & $0.44 \pm 0.02$ & $57 \pm 0.04$ \\
\hline $\mathrm{N}$-base bare & $7.5 \pm 0.8$ & $23.5 \pm 2.4$ & $0.45 \pm 0.01$ & $68 \pm 0.02$ \\
\hline $\mathrm{N}$-base ITO & $7.4 \pm 1.0$ & $27.0 \pm 2.2$ & $0.43 \pm 0.01$ & $63 \pm 0.03$ \\
\hline $\mathrm{N}$-base $\mathrm{Al}_{2} \mathrm{O}_{3}$ & $10.1 \pm 0.7$ & $33.8 \pm 0.8$ & $0.46 \pm 0.01$ & $70 \pm 0.05$ \\
\hline $\mathrm{N}$-base $\mathrm{SiN}_{\mathbf{x}}$ & $9.5 \pm 0.9$ & $28.9 \pm 2.9$ & $0.45 \pm 0.01$ & $72 \pm 0.02$ \\
\hline $\mathrm{N}$-base $\mathrm{SiO}_{2}$ & $8.3 \pm 0.8$ & $25.1 \pm 2.0$ & $0.46 \pm 0.01$ & $72 \pm 0.03$ \\
\hline
\end{tabular}

To prove that the increase in efficiency upon applying a coating is not due to differences between wafers, bare p-type base samples, which were first electrically analyzed, were passivated with an $\mathrm{Al}_{2} \mathrm{O}_{3}$ coating. $\mathrm{JV}$ measurements were recorded again after coating, and indeed revealed a clear increase in the overall efficiency (Figure 5.7). 


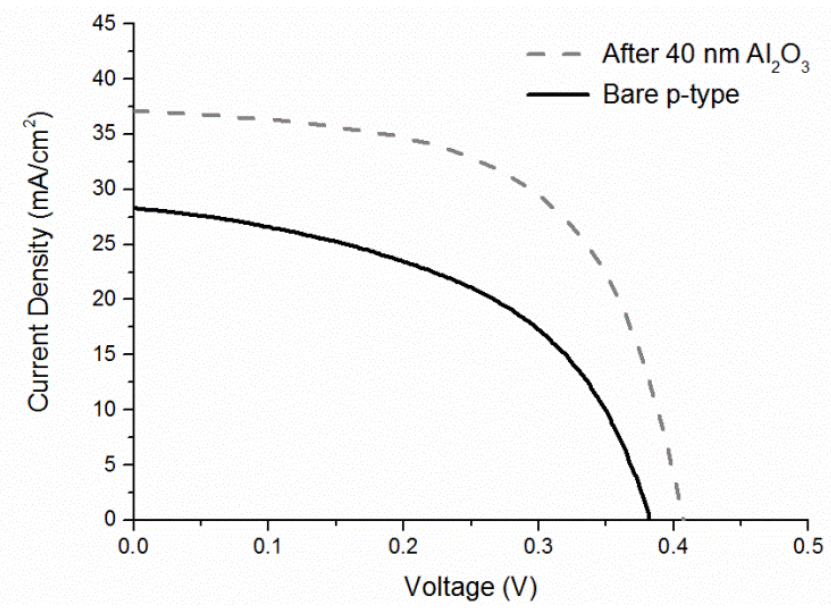

Figure 5.7: $J V$ measurements on a bare pillared sample (black line) and after ALD deposition of $40 \mathrm{~nm} \mathrm{Al}_{2} \mathrm{O}_{3}$ on this specimen (dashed line).

Such $J V$-measurements before and after application of a coating were not possible for the LPCVD materials, since the required temperature for LPCVD processes (well above $400{ }^{\circ} \mathrm{C}$ ) causes severe roughening of the aluminum contact, which results in short-circuiting and hence destroys the junction. ${ }^{[31]}$ When comparing $\mathrm{p}$ - and $\mathrm{n}$-type base specimen, $\mathrm{p}$-type base samples gave a higher current density for identical coatings, whereas for $n$-type wafers the $V_{O C}$ and $F F$ were higher. The increase in $J_{S C}$ for coated p-type base samples is higher than for coated $n$-type base samples, for which reason the $p$-type specimen gave a higher overall efficiency. The large differences between the recorded $V_{O C}$ and $J_{S C}$ between the two different base wafers for identical applied coatings is hard to explain, although this is probably caused by experimental parameters, such as different doping techniques (LPCVD vs. SSD), base concentrations (1-10 (p-type) vs 5-10 $\Omega \mathrm{cm}$ (n-type)) and doping profiles (Figure 5.8). 


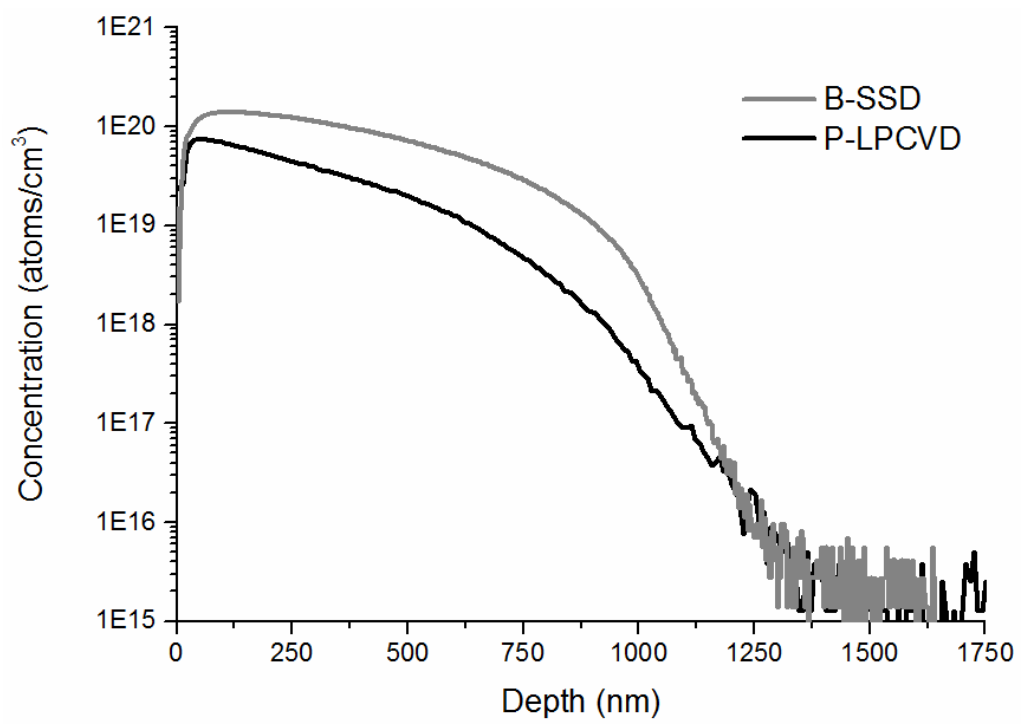

Figure 5.8: $J V$ measurements on A) p- and B) $n$-type base micropillars with radial $p / n$ junctions, with and without coating materials.

Both LPCVD materials $\mathrm{SiO}_{2}$ and $\mathrm{SiN}_{\mathrm{x}}$ show a non-uniform coating on the sidewalls of the pillars (see Figure 5.4). At the topside of the pillars, the exact thickness and high uniformity of the AR-coating are crucial in order to obtain perfect AR behavior (which is accomplished in this work, see 5.3.2). However, for the sidewalls these aspects - i.e. coating thickness and uniformity - are of lesser importance, since, as we mentioned above, light entering the space in between pillars will, after several scattering events, finally be absorbed by the pillars. At the sidewalls of the pillars, the contribution of the coating therefore mostly will be passivation. For this, a thickness of a few nanometers is sufficient, without stringent uniformity requirements. In fact, the explored LPCVD coatings fulfill the specifications to act as AR coating on the topside of the pillars and as passivation layer at the sidewalls, which is clear when taken the following arguments into account. The reflection is reduced by the pillar arrays and $34 \%$ of the light is reflected 'normally' (see section 5.3.1). If the coating only functioned as anti-reflection coating, the overall efficiency could only be $8.2 \%$ and $10.1 \%$ for $p$ - and n-type base, respectively. From Table 5.2 it becomes clear, that the overall efficiency gain is more. This implies indirectly that the coating also functioned as a passivation layer. ALD materials are 
formed by self-limiting deposition cycles, which is evidenced in perfect conformality on the topside and sidewalls of the pillar array. Therefore, ALD is a good way to both passivate and create an AR-coating on high aspect ratio micropillars, which follows from the electrical characterization (see Table 5.2). $\mathrm{Al}_{2} \mathrm{O}_{3}, \mathrm{SiO}_{2}$ and $\mathrm{SiN}_{\mathrm{x}}$ have a double function (two-fold coating), but a three-fold function of a coating is also an option, i.e. conductive oxides. Unfortunately, the composition of ITO is not constant over the pillar height (see section 3.2). Both effects counteract the theoretical efficiency improvement of the ITO-coating, and as a result, the performance of ITO-coated solar cells is only improved marginally with respect to bare pillar arrays. ITO as a passivation and AR-coating is not successful; series resistances were not lowered and the $J_{S C}$ output was only increased slightly, whereas the $V_{O C}$ even decreased.

\subsection{Conclusions}

In this work, the deposition and functionality of different anti-reflective and passivation coatings was investigated. Using simulations, the optimal thickness for these materials were predicted and empirically tested on flat silicon solar cells. Four different layers were then grown on radial $p / n$ junctions (both $p$ - and $n$-type base) using different techniques (ALD of $\mathrm{Al}_{2} \mathrm{O}_{3}$, LPCVD of $\mathrm{SiO}_{2}$ and $\mathrm{SiN}_{\mathrm{x}}$, and sputtering of ITO) and analyzed and characterized by HR-SEM imaging and $J V$-measurements. The ALD deposition was conformal over the height of the pillar, whereas for the LPCVD and sputtering deposition, gradients were visible. For LPCVD the layer at the top of pillar was thinner than the bottom side, whereas the sputtering technique was not able to fill the scalloping (caused by DRIE, Bosch process). For ITO it was visible that less indium was present further down the pillar, meaning it probably lost its conductive property. $J V$ measurements showed that $\mathrm{Al}_{2} \mathrm{O}_{3}$ improved the overall efficiency the most (6.1 to $13.4 \%$ for $p$-type and 7.4 to $10.1 \%$ for n-type), whereas ITO was the only coating to show a (small) decrease, compared to flat samples. This is probably due to the lack of indium atoms, caused by the sputtering of ITO of high aspect ratio structures. It can be 
concluded that atomic layer deposition is most suitable for the growth of these anti-reflective and passivation layers on high aspect ratio structures.

\section{Acknowledgements}

Wouter Vijselaar is greatly acknowledged for his ideas, discussions and ALD, LPCVD and JV experiments in this chapter. Roald Tiggelaar is thanked for corrections and discussions on this chapter. Henk van Wolferen is acknowledged for the FIB experiments. Mark Smithers is thanked for the HR-SEM images. 


\subsection{References}

[1] J. Zhao, A. Wang, P. P. Altermatt, S. R. Wenham, M. A. Green, Sol. Energy Mater. Sol. Cells, 1996, 41-42, 87-99.

[2] A. Shah, P. Torres, R. Tscharner, N. Wyrsch, H. Keppner, Science, 1999, 285, 692-698.

[3] T. Saga, NPG Asia Mater, 2010, 2, 96-102.

[4] E. Garnett, P. Yang, Nano Lett., 2010, 10, 1082-1087.

[5] K. Q. Peng, S. T. Lee, Adv. Mater., 2011, 23, 198-215.

[6] A. I. Hochbaum, P. Yang, Chem. Rev., 2010, 110, 527-546.

[7] S. Y. Reece, J. A. Hamel, K. Sung, T. D. Jarvi, A. J. Esswein, J. J. H. Pijpers, D. G. Nocera, Science, 2011, 334, 645-648.

[8] E. L. Warren, H. A. Atwater, N. S. Lewis, The Journal of Physical Chemistry C, 2013, 118, 747-759.

[9] K. S. Joya, Y. F. Joya, K. Ocakoglu, R. Van De Krol, Angew. Chem. Int. Ed., 2013, 52, 10426-10437.

[10] B. M. Kayes, H. A. Atwater, N. S. Lewis, J. Appl. Phys., 2005, 97, 114302.

[11] L. Tsakalakos, J. Balch, J. Fronheiser, B. A. Korevaar, O. Sulima, J. Rand, Appl. Phys. Lett., 2007, 91, 233117.

[12] Z. Yu, H. Gao, W. Wu, H. Ge, S. Y. Chou, J. Vac. Sci. Technol. B, 2003, 21, 2874-2877.

[13] H. Xu, N. Lu, D. Qi, J. Hao, L. Gao, B. Zhang, L. Chi, Small, 2008, 4, 1972-1975.

[14] G. Dingemans, N. M. Terlinden, D. Pierreux, H. B. Profijt, M. C. M. van de Sanden, W. M. M. Kessels, Electrochem. Solid-State Lett., 2011, 14, H1-H4.

[15] B. Hoex, J. J. H. Gielis, M. C. M. van de Sanden, W. M. M. Kessels, J. Appl. Phys., 2008, 104, 113703.

[16] R. B. M. Girisch, R. P. Mertens, R. F. De Keersmaecker, Electron Devices, IEEE Transactions on, 1988, 35, 203-222.

[17] A. G. Aberle, S. Glunz, W. Warta, Sol. Energy Mater. Sol. Cells, 1993, 29, 175-182.

[18] J. Schmidt, EU PVSEC Proceedings, 2012, DOI: 10.4229/25thEUPVSEC2010-2AO.1.6, $1130-1133$.

[19] D. R. Kim, C. H. Lee, P. M. Rao, I. S. Cho, X. Zheng, Nano Lett., 2011, 11, 2704-2708.

[20] A. D. Mallorquí, E. Alarcón-Lladó, I. C. Mundet, A. Kiani, B. Demaurex, S. De Wolf, A. Menzel, M. Zacharias, A. Fontcuberta i Morral, Nano Res., 2015, 8, 673-681.

[21] M. Faustini, L. Nicole, C. Boissière, P. Innocenzi, C. Sanchez, D. Grosso, Chem. Mater., 2010, 22, 4406-4413.

[22] A. Szeghalmi, M. Helgert, R. Brunner, F. Heyroth, U. Gösele, M. Knezl, Appl. Opt., 2009, 48, 1727-1732.

[23] G. Dingemans, R. Seguin, P. Engelhart, M. C. M. van de Sanden, W. M. M. Kessels, Phys. Status Solidi RRL, 2010, 4, 10-12.

[24] F. Duerinckx, J. Szlufcik, Sol. Energy Mater. Sol. Cells, 2002, 72, 231-246.

[25] H. MäcKel, R. Lüdemann, J. Appl. Phys., 2002, 92, 2602-2609.

[26] R. Elbersen, R. M. Tiggelaar, A. Milbrat, G. Mul, H. Gardeniers, J. Huskens, Adv. Energy Mater., 2015, 5, 1401745.

[27] R. Hulstrom, R. Bird, C. Riordan, Solar Cells, 1985, 15, 365-391.

[28] S. C. Baker-Finch, K. R. McIntosh, Photovoltaic Specialists Conference (PVSC), 2010 35th IEEE, 2010.

[29] G. Dingemans, Ph.D. thesis, Technical University Eindhoven, 2011.

[30] F. Duerinckx, J. Szlufcik, Sol. Energy Mater. Sol. Cells, 2002, 72, 231-246.

[31] J. Bullock, D. Yan, Y. Wan, A. Cuevas, B. Demaurex, A. Hessler-Wyser, S. De Wolf, J. Appl. Phys., 2014, 115, 163703. 


\section{Chapter 6}

\section{Spatioselective Electrochemical and}

Photoelectrochemical Functionalization of Silicon Microwires with Axial $\mathbf{p} / \mathbf{n}$ Junctions

A powerful method is presented to functionalize selectively the top and bottom of semiconductor wires with axial junctions. A light switch is used to address individual segments electrochemically for modification. The principle is demonstrated by deposition of platinum on the bottom $p$ type and silver on the top $n^{+}$type of silicon microwires with an axial junction. Between the dual functionalizations, an unmodified band is observed which is related to the depletion layer of the $\mathrm{p} / \mathrm{n}$ interface.

This chapter has been adapted from: A. Milbrat, R. Elbersen, R. Kas, R.M. Tiggelaar, J.G.E. Gardeniers, J. Huskens, G. Mul, "Spatioselective Electrochemical and Photoelectrochemical Functionalization of Silicon Microwires with Axial p/n Junctions", Adv. Mater., 2015, accepted 


\subsection{Introduction}

Semiconductor wires and wire arrays of nano- to micrometer size have unique optical, electronic, electrochemical, thermal, mechanical and magnetic properties. ${ }^{[1]}$ Therefore, they are one of the most considered structures for electronic, sensor, photonic, thermoelectric, photovoltaic, photoelectrochemical and battery applications. ${ }^{[1-5]}$ Semiconductor wires often require functionalization, for example, by deposition of catalysts, ${ }^{[6]}$ additional semiconductors,${ }^{[7]}$ grafting with organic molecules ${ }^{[8]}$ or polymers, ${ }^{[9]}$ in order to tailor their properties for the targeted application. However, these modifications are commonly performed across the entire surface of the wires. Janus-type wires on the other hand, of which the top and bottom segments have different functionalizations, could provide access to new types of devices or improve the functionality of existing ones. ${ }^{[10]}$ Many applications, such as sensors, ${ }^{[11]}$ tunnel field-effect transistors, ${ }^{[12-15]}$ light-emitting diodes, ${ }^{[16,17]}$ photovoltaics ${ }^{[18-20]}$ and photonics, ${ }^{[16,21]}$ require or would benefit from an axial homo- or heterojunction and electrical communication between the segments.

The feasibility of creating bi-functionalized semiconductor wires and wire arrays has been reported previously in literature for their use in photocatalysis, ${ }^{[22-24]}$ microfluidics ${ }^{[25,26]}$ or as super hydrophobic surfaces. ${ }^{[27]}$ However, the field of Janus wires is not yet extensively explored, mainly because their preparation is complex and requires several steps. So far, bi-functionalized wires have been created by physically masking the part of the wire that was to remain unmodified. Typically, wires were embedded completely in a polymeric material first, which was subsequently etched back to expose parts of the wires for further functionalization. This masking process makes it difficult to control the functionalization boundary precisely, reproducibly, and homogeneously across an array, which is particularly important for, for example, axially heterostructured wires where a small misalignment could lead to a failure of the device. 
Herein, we show a method for the direct and spatioselective bi-functionalization of semiconductor microwires, which employs the different electronic properties of an axial junction in dark and under illumination. Exemplary, we have used silicon microwires with an axial $\mathrm{n}^{+} / \mathrm{p}$ junction.

Figure 6.1 schematically shows the concept. It starts from a silicon microwire that has an axial $n^{+} / p$ junction, prepared by the $n$-doping of a flat $p$-base wafer followed by dry etching to create the microwires. We anticipated that the junction would induce diode-like behavior when a negative potential is applied to the p-region, preventing electrons to pass from bottom to top. This would allow the selective functionalization of the bottom segment by reductive electrodeposition. The feasibility of this hypothesis is tested here by the electrodeposition of Pt nanoparticles from a hexachloroplatinic acid precursor solution (Figure 6.1a). Under illumination, the junction induces electrons to migrate to the $\mathrm{n}^{+}-\mathrm{Si}$ surface and allow its selective functionalization, which will be demonstrated here by the electrodeposition of silver (Figure 6.1b and 6.1c). The holes accumulated in the $\mathrm{p}$-Si part recombine with electrons supplied by an external source, i.e. by applying a negative bias to the base silicon, or oxidize $\mathrm{p}$-Si surface. Performing both steps consecutively results in dually and spatioselectively functionalized arrays of microwires. Platinum and silver were chosen for a proof of concept, because these metals form Ohmic contacts to the p-type and $\mathrm{n}^{+}$-type silicon, respectively. As Figure 6.1 indicates, we observed an unmodified band between the functionalized segments in the final wires. We provide here analytical data and theory, as well as a preliminary explanation for this phenomenon. 


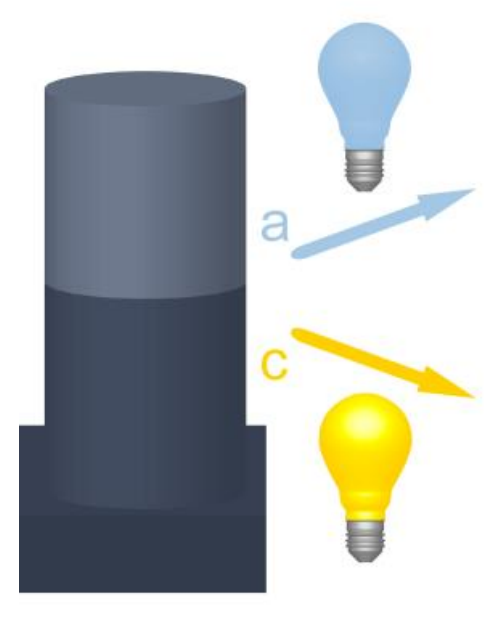

light on light off

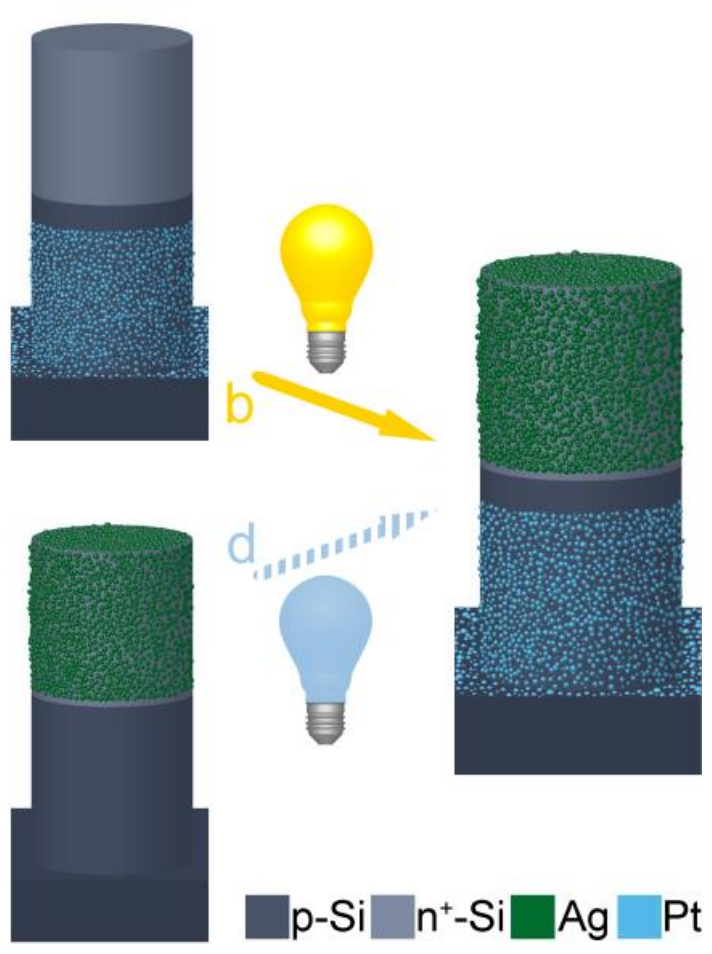

Figure 6.1: Schematic illustration of the spatioselective functionalization process of silicon microwires with an axial $\mathrm{n}^{+} / \mathrm{p}$ junction: an initial silicon microwire (left) is functionalized by the electrochemical deposition of Pt in the dark on the p Si part (a) and of $\mathrm{Ag}$ under illumination on the $\mathrm{n}^{+}$-Si part $(\mathrm{b}, \mathrm{c})$. Sequential deposition of $\mathrm{Pt}$ and $\mathrm{Ag}$ leads to a bifunctionalized silicon microwire (right) in which a depletion zone is visible between the functionalized areas. Step (d) provides an optional, but here unexplored, route.

\subsection{Materials and methods}

\subsubsection{Fabrication of axial $\mathrm{p} / \mathrm{n}$ junctions in silicon micropillar arrays}

The fabrication process for the axial $p / n$ junction is schematically shown in Figure 6.2. Boron doped, p-type silicon substrates ((100) oriented, resistivity 5-10 $\Omega \mathrm{cm}, 100 \mathrm{~mm}$ diameter, $525 \mu \mathrm{m}$ thickness, single side polished, Okmetic Finland) were cleaned by immersion in $100 \%$ nitric acid $\left(\mathrm{HNO}_{3}\right)$ (2x $\left.5 \mathrm{~min}\right)$ and in fuming $69 \%$ nitric acid (15 min), followed by quick dump rinsing in de-mineralized (DI) water, immersion in $1 \%$ aqueous hydrofluoric (HF) acid to remove the formed oxide shell and another quick dump rinsing cycle. After spin drying the wafers, $100 \mathrm{~nm}$ thick silicon-rich silicon nitride (SiRN) was 
deposited using low-pressure chemical vapor deposition (LPCVD) on both sides of the wafer, removed from the front side with reactive ion etching (Adixen AMS100DE; octafluorocyclobutane $\left(\mathrm{C}_{4} \mathrm{~F}_{8}\right)$ and methane $\left(\mathrm{CH}_{4}\right)$ ), followed by an oxygen plasma treatment and piranha cleaning (mixture of concentrated sulfuric acid and $30 \%$ aqueous hydrogen peroxide, $3: 1(\mathrm{v} / \mathrm{v})$, $20 \mathrm{~min}$ ) to remove any contamination (i.e. fluorocarbon traces from the DRIE-process). Subsequently, the front side was covered by a phosphorus spin-on-glass (P-SOG, Filmtronics P509), spun at $6000 \mathrm{rpm}$ for $30 \mathrm{~s}$, and annealed at $1100{ }^{\circ} \mathrm{C}$ for $60 \mathrm{~min}$. The remaining $\mathrm{P}-\mathrm{SOG}$ and SiRN layers were removed by immersion in buffered hydrogen fluoride (1:7, BHF) for $10 \mathrm{~min}$, washed with DI water and dried with a nitrogen flow. By means of standard UV-lithography, six $2 \times 2 \mathrm{~cm}^{2}$ areas with an array of microwires (specifications: diameter $4 \mu \mathrm{m}$, spacing $2 \mu \mathrm{m}$, hexagonally stacked with a packing density of $35 \%$ ) were defined in photoresist (Olin 907-17), and post-baked for $10 \mathrm{~min}$ at $120^{\circ} \mathrm{C}$ after exposure and development. The photoresist acted as a mask layer during cryogenic $\left(-100^{\circ} \mathrm{C}\right)$ deep reactive ion etching (DRIE; Adixen AMS100SE, $1000 \mathrm{~W})$ of silicon, using sulfur hexafluoride $\left(\mathrm{SF}_{6}, 200 \mathrm{sccm}\right)$ for etching silicon and $\mathrm{O}_{2}(30 \mathrm{sccm})$ for passivating the sidewalls. The height of the wires was determined by the etch duration, which was set to $2 \mathrm{~min}$, resulting in wire heights of approximately $7 \mu \mathrm{m}$. After etching, the photoresist mask was stripped off with oxygen plasma, followed by piranha cleaning (20 min), rinsing with DI water and drying with nitrogen. Prior to selective electrodeposition experiments, the sample was immersed in $1 \%$ aqueous hydrofluoric (HF) acid solution for at least $1 \mathrm{~min}$ to remove the silicon oxide shell, washed by quick dump rinsing in de-mineralized (DI) water, spin dried and the backside sputter-coated with an $1 \mu \mathrm{m}$ thick aluminum/silicon alloy (99\% Al, 1\% Si) (Oxford PL 400, $7000 \mathrm{~W}$ ) to create a low resistance Ohmic contact. 


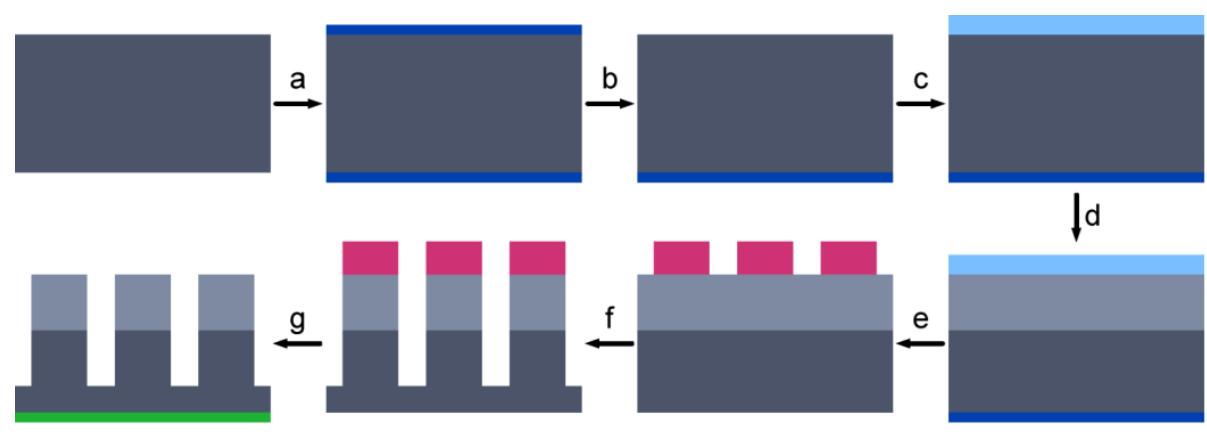

$\begin{array}{llll}\text { p-Si } & n^{+}-S i & \begin{array}{l}\text { Silicon-rich } \\ \text { silicon nitride }\end{array} & \begin{array}{l}\text { phosphorus } \\ \text { spin-on-glass }\end{array} \text { photoresist Al/Si alloy }\end{array}$

Figure 6.2: Schematic illustration of the synthesis of silicon microwires with axial $n^{+} / p$ junctions. a) Low-pressure chemical vapor deposition (LPCVD) of silicon-rich silicon nitride (SiRN) on $p$ type silicon substrates. b) Removal of SiRN from the front side by reactive ion etching (RIE). c) Spin coating of phosphorus spin on glass (P-SOG) at $6000 \mathrm{rpm}$ for $30 \mathrm{~s}$. d.) Thermal phosphorus drive in at $1100^{\circ} \mathrm{C}$ for $60 \mathrm{~min}$. e) Definition of microwire arrays by standard UV lithography. f) Cryogenic $\left(-100{ }^{\circ} \mathrm{C}\right)$ deep reactive ion etching (DRIE). g) Removal of photoresist and silicon oxide and sputtering of aluminum/silicon alloy $(99 \% \mathrm{Al}$, $1 \% \mathrm{Si})$.

\subsubsection{Electrodeposition of platinum and silver}

Prior to the electrodeposition experiments, specimens were immersed in $1 \%$ aqueous hydrofluoric acid (HF) solution to remove the native oxide, followed by creation of a $1 \mu \mathrm{m}$ thick aluminum/silicon alloy ( $99 \% \mathrm{Al}, 1 \% \mathrm{Si}$ ) by sputtering on the backside, to function as a low-resistance Ohmic contact. Broken out microwire arrays $\left(2 \times 2 \mathrm{~cm}^{2}\right)$ were used full sized for Pt deposition or further broken in 4 pieces (approximately $1 \times 1 \mathrm{~cm}^{2}$ ) for $\mathrm{Ag}$ deposition.

Platinum was deposited on the bottom $\mathrm{p}$-Si segments from an aqueous solution of $5 \mathrm{mM}$ hexachloroplatinic(IV) acid $\left(\mathrm{H}_{2} \mathrm{PtCl}_{6}\right)$ and $0.5 \mathrm{M}$ sodium sulfate $\left(\mathrm{Na}_{2} \mathrm{SO}_{4}\right)$, potentiostatically at $-0.7 \mathrm{~V}$. Charge densities of $130 \mathrm{mC} / \mathrm{cm}^{2}$ were supplied. Silver was deposited on the top $n$-Si segments from an aqueous solution of $1 \mathrm{mM}$ silver nitrate $\left(\mathrm{AgNO}_{3}\right)$ and either $0.5 \mathrm{M} \mathrm{Na}_{2} \mathrm{SO}_{4}$, $0.005 \mathrm{M} \mathrm{H}_{2} \mathrm{SO}_{4}$ or $0.5 \mathrm{M} \mathrm{H}_{2} \mathrm{SO}_{4}$ as the electrolyte, with a pH of 5.3, 2.4 and 0.6 , respectively. Voltages applied were $-0.1 \mathrm{~V},+0.1 \mathrm{~V}$ and $+0.3 \mathrm{~V}$. The deposition was discontinued when the charge density amounted to $25 \mathrm{mC} / \mathrm{cm}^{2}$. 
A solar simulator with a $300 \mathrm{~W}$ xenon lamp and an air mass 1.5 global filter was used as a light source for experiments performed under illumination. The intensity of the simulated sunlight was adjusted to 2 suns $\left(200 \mathrm{~mW} / \mathrm{cm}^{2}\right)$ with a standard reference silicon solar cell and partially cut off at $570 \mathrm{~nm}$ with a cut-on filter (Newport) to avoid significant homogeneous photodecomposition of $\mathrm{AgNO}_{3}$ in solution by shorter wavelengths. The custom-made Teflon-based reactors with a volume of about $8 \mathrm{ml}$ were equipped with an optical glass window and were open to the environment. Active surface areas applied were $3.14 \mathrm{~cm}^{2}$ for platinum and $0.28 \mathrm{~cm}^{2}$ for silver deposition, respectively, as determined by the O-rings of the reactors. A standard three-electrode system with a platinum mesh counter electrode was used. A potentiostat (PAR, VersaStat 4) served as a power source. All applied potentials are reported versus an $\mathrm{Ag} / \mathrm{AgCl}$ ( $3 \mathrm{M} \mathrm{NaCl}$, BASi MF-2052) reference electrode, and all electrochemical experiments were performed at room temperature.

\subsubsection{Scanning electron microscopy and energy-dispersive X-ray spectroscopy}

High-Resolution Scanning Electron Microscopy (HR-SEM) images were taken with a Philips FEI XL30 FEG-ESEM equipped with a Secondary Electron (SE) detector or a FEI Sirion FEG-SEM with a Through the Lens Detector (TLD), operated at typical acceleration voltages of $10 \mathrm{kV}$.HR-SEM in combination with Energy-Dispersive X-ray spectroscopy (EDX). EDX mapping was performed with a MERLIN FEG HR-SEM with an inlens SE detector and an EDX Spectrometer, $80 \mathrm{~mm}^{2}$ detector.

\subsection{Results and discussion}

Figure 6.3a shows platinum particles, electrodeposited in the dark, selectively on the p-type base and bottom segments of the axially doped silicon microwires. Clearly, the top $\mathrm{n}^{+}$-type segments have remained unmodified. A control experiment, performed at identical electrochemical conditions but applied to $\mathrm{p}$-Si microwires without phosphorus $\mathrm{n}^{+}-\mathrm{Si}$ top segments, yielded completely covered microwires (Figure 6.3b). 
This confirms our concept of the blocking of the electron flow to the $\mathrm{n}^{+}-\mathrm{Si}$ top parts in the dark by the diode present in the axially doped microwires. The metal was deposited as particles and not as a continuous film, which is consistent with the theory of Volmer-Weber island growth. ${ }^{[15]}$ In both samples shown in Figure 6.3, the density of the particles is higher at the top of the functionalized segment compared to the bottom of the microwires, which is tentatively attributed to diffusion limitation of the precursor in between the microwires at these densely packed arrays.
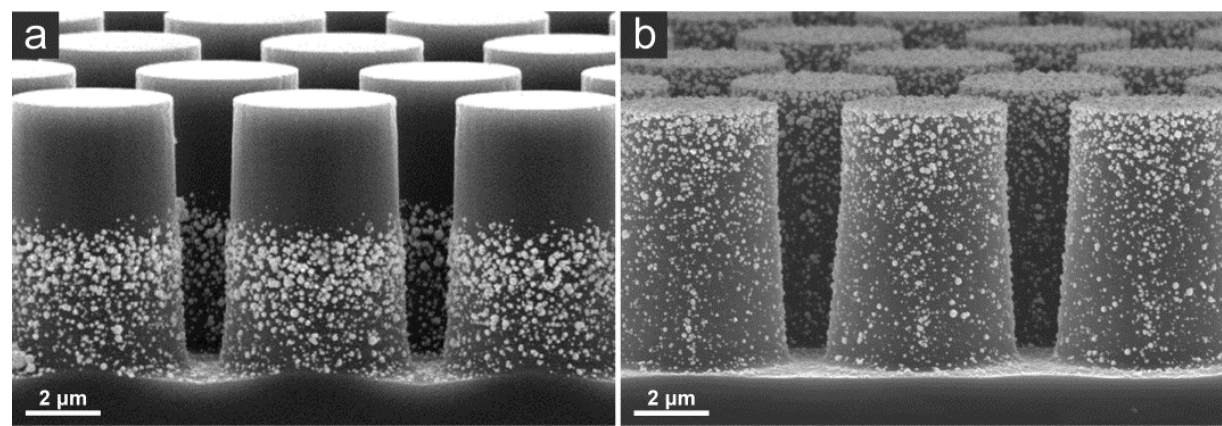

Figure 6.3: Cross-sectional SEM images of a) Si microwires with a $\mathrm{n}^{+} / \mathrm{p}$ junction and b) $\mathrm{p}$-Si microwires after the electrodeposition of Pt particles at $0.7 \mathrm{~V}$ and $130 \mathrm{mC} / \mathrm{cm}^{2}$ in the dark from a solution of $5 \mathrm{mM} \mathrm{H}_{2} \mathrm{PtCl}_{6}$ and $0.5 \mathrm{M} \mathrm{Na}_{2} \mathrm{SO}_{4}$.

Figure 6.4 shows silver particles, electrodeposited selectively on the top $\mathrm{n}^{+}-\mathrm{Si}$ segments under illumination (Figure 6.4a) and semi-selectively on the p-type base and bottom segments in dark (Figure 6.4b) of the axially doped silicon microwires. Selective photo-supported electrodeposition of silver on the top of the wires at the $\mathrm{n}^{+}-\mathrm{Si}$ segments was achieved in $0.5 \quad \mathrm{M} \mathrm{H}_{2} \mathrm{SO}_{4}$ Electrodeposition of $\mathrm{Ag}$ in the dark yielded major deposition on the bottom $\mathrm{p}$-Si segments and minor deposition on the top $\mathrm{n}$-Si segments (Figure 6.4b). We assume that the formation of a Schottky junction between $\mathrm{Ag}$ and $\mathrm{p}$-Si close to the $n^{+} / p$ junction caused a "leak" of electrons through the $n^{+} / p$ junction by a partial overlap of both junction depletion layers. Thus, an Ohmic contact between semiconductor wire segments and functionalized material is required to achieve high selectivity in functionalization. 

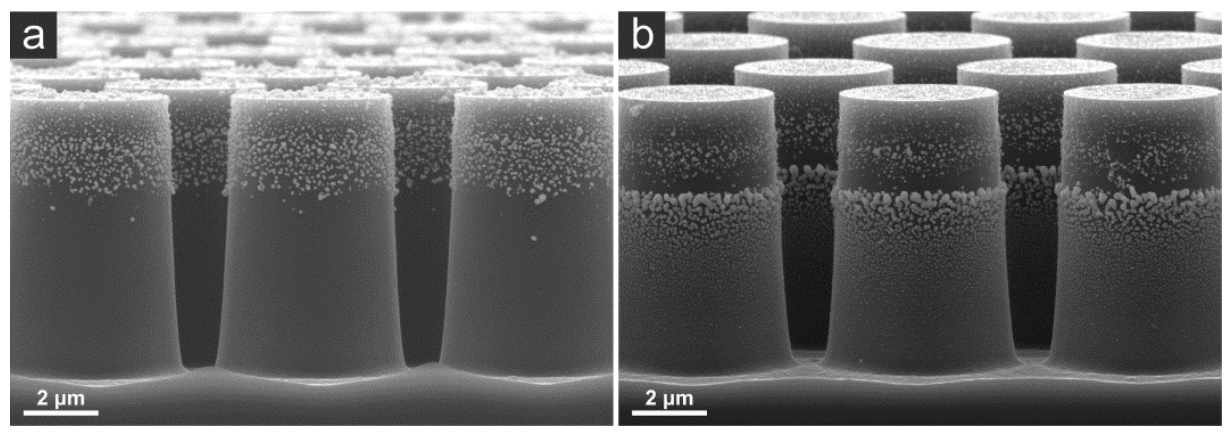

Figure 6.4: Cross-sectional SEM images of silicon microwires with an axial $\mathrm{n}^{+} / \mathrm{p}$ junction after the electrodeposition of $\mathrm{Ag}$ particles at $25 \mathrm{mC} / \mathrm{cm}^{2}$ from a solution of $1 \mathrm{mM} \mathrm{AgNO}_{3}$ and $0.5 \mathrm{M} \mathrm{H}_{2} \mathrm{SO}_{4}$ at a) $+0.3 \mathrm{~V}$ under illumination and b) $0.3 \mathrm{~V}$ in the dark.

To realize bi-functionalized silicon microwires, we first performed the electrodeposition of platinum in the dark, followed by an immersion of the sample into a $1 \%$ aqueous HF solution ( $30 \mathrm{~s}$ ), and subsequently conducted the photo-induced electrodeposition of silver. This order is necessary in our case because otherwise $\mathrm{Ag}$ is galvanically replaced by $\mathrm{Pt}$ from the $\mathrm{Pt}$ precursor solution. Figure 6.5 shows cross-sectional SEM images of such bi-functionalized silicon microwires with an axial $\mathrm{n}^{+} / \mathrm{p}$ junction. The presence of silver on the top parts and platinum on the bottom parts of the microwires shown in Figure 6.5a, was confirmed by SEM/EDX mapping (Figure 6.5b). The deposition boundary of silver is observed at $2.4 \mu \mathrm{m}$ and that of platinum at $3.3 \mu \mathrm{m}$ from the top of the microwire. In between the metal-covered areas, the microwires exhibit an unmodified band with a width of approximately $0.9 \mu \mathrm{m}$. In the following, these observations will be discussed in more detail. 

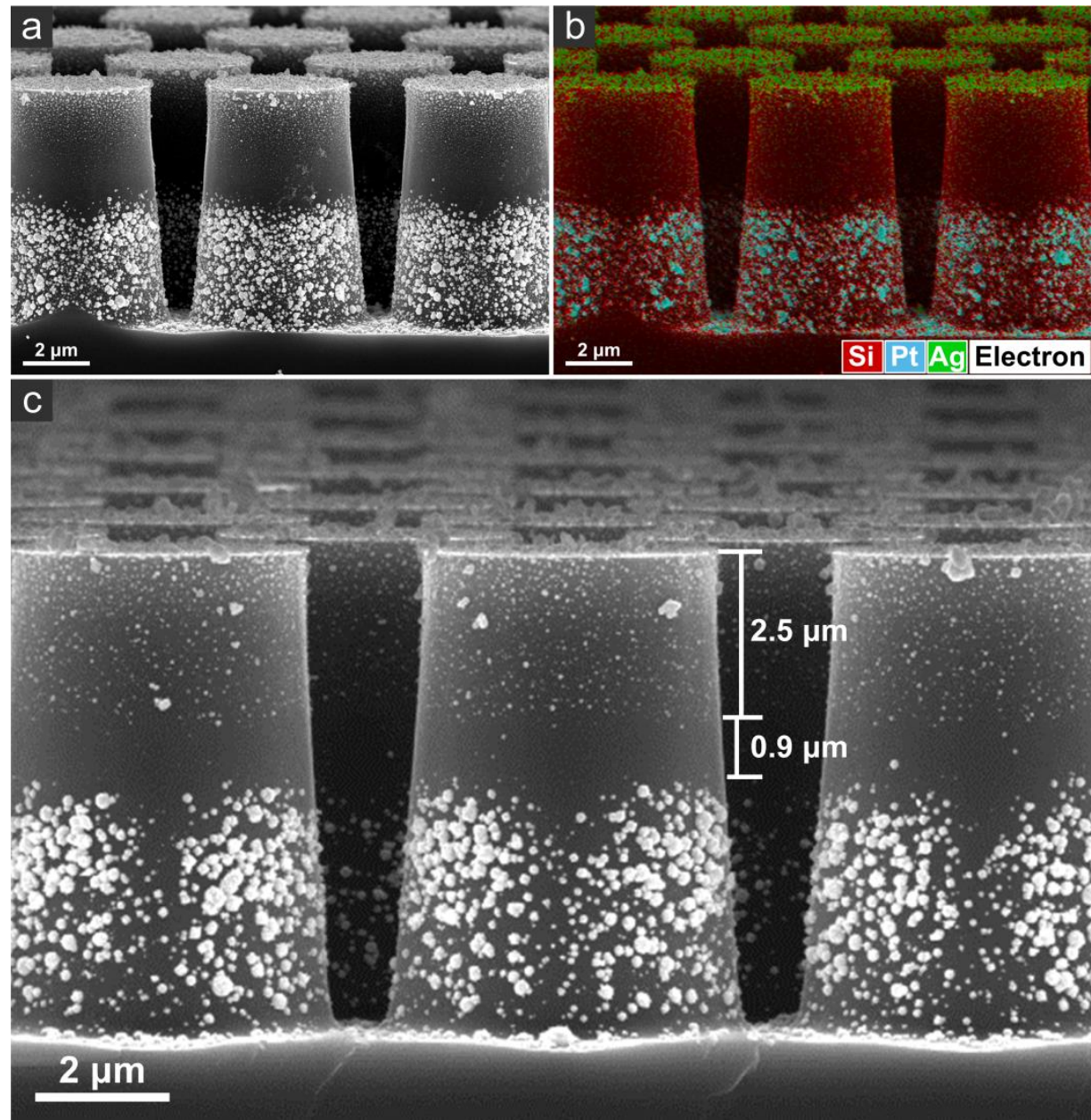

Figure 6.5: Cross-sectional SEM images $(a, c)$ and SEM/EDX mapping (b) of silicon microwires with an axial $\mathrm{n}^{+} / \mathrm{p}$ junction after the electrodeposition of Pt particles at $-0.7 \mathrm{~V}$ and $130 \mathrm{mC} / \mathrm{cm}^{2}$ in the dark from a solution of $5 \mathrm{mM} \mathrm{H}_{2} \mathrm{PtCl}_{6}$ and $0.5 \mathrm{M} \mathrm{Na}_{2} \mathrm{SO}_{4}$, followed by the electrodeposition of $\mathrm{Ag}$ particles at $25 \mathrm{mC} / \mathrm{cm}^{2}$ under illumination from a solution of $1 \mathrm{mM} \mathrm{AgNO}_{3}$ in a,b) $5 \mathrm{mM} \mathrm{H}_{2} \mathrm{SO}_{4}$ electrolyte at $-0.1 \mathrm{~V}$ and c) $0.5 \mathrm{M} \mathrm{Na}_{2} \mathrm{SO}_{4}$ electrolyte at $+0.3 \mathrm{~V}$.

The mechanism of our concept of dual functionalization of axially doped microwires and the occurrence of the unmodified band in between the metalcovered areas can be explained using the theory of a $\mathrm{p} / \mathrm{n}$ junction. Figure $6.6 \mathrm{a}$ shows band edges of a $\mathrm{p}$ - and highly $\mathrm{n}$-doped semiconductor (silicon in our case) with its Fermi levels. Connecting the $\mathrm{p}$ - and $\mathrm{n}$-type parts results in an electron flow from filled states in the $\mathrm{n}$-Si conduction band to empty states of 
the $\mathrm{p}$-Si valence band until the Fermi level of both silicon types equalize. A depletion layer is thus formed, and this causes bending of the p-Si and n-Si bands (Figure 6.6b). The depleted areas in $\mathrm{n}-\mathrm{Si}$ and $\mathrm{p}-\mathrm{Si}$ get positively and negatively charged, respectively. Due to the higher doping level of $\mathrm{n}-\mathrm{Si}$, the depletion layer penetrates mostly into p-Si.
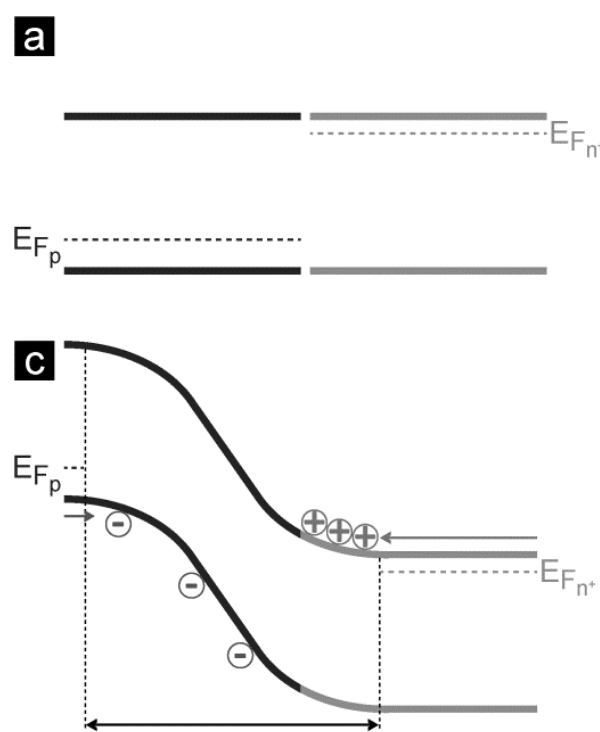

- $p$-Si band edge $\quad E_{F_{p}} \cdots p$-Si Fermi leve - $\mathrm{n}^{+}$-Si band edge $\quad \cdots E_{\mathrm{F}_{\mathrm{n}^{+}}} \mathrm{n}^{+}-\mathrm{Si}$ Fermi level

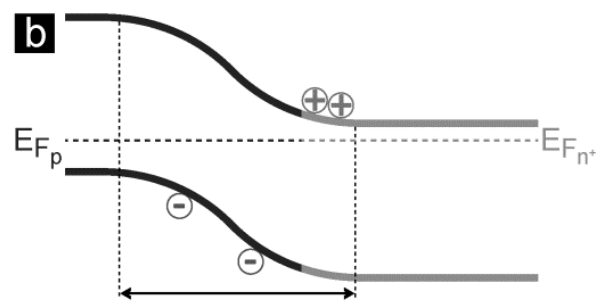

d

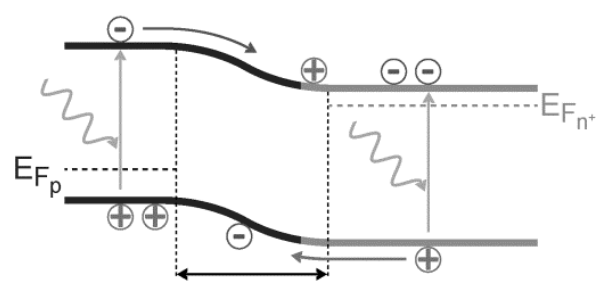

$\odot$ electron $\leftrightarrow$ depletion layer

$\oplus$ hole Li photoexcitation

Figure 6.6: Schematic illustration of $\mathrm{n}^{+} / \mathrm{p}$-Si band diagrams a) before contact, b) in contact at equilibrium, c) reverse-biased, d) under illumination.

The orientation of the diode in our concept is such that it is reverse-biased when the base is put at a negative potential. Consequently, the potential barrier in the junction prevents a significant amount of electrons to pass the junction in the dark (Figure 6.6c). These electrons, supplied by an external source, occupy the empty states in the $\mathrm{p}$-Si valence band and thereby enlarge the depletion layer into the bulk or migrate to the surface of the p-type semiconductor, where they induce its selective functionalization, which is in our case the electrodeposition of platinum. We assume that in this case only electrons outside of the depletion layer are available for the reduction of $\mathrm{Pt}$ precursor at the silicon surface. 
Upon illumination, photoexcited electrons drift across the junction from the $\mathrm{p}$-Si conduction band into the $\mathrm{n}^{+}-\mathrm{Si}$ conduction band while holes migrate from the $\mathrm{n}^{+}-\mathrm{Si}$ valence band to the $\mathrm{p}-\mathrm{Si}$ valence band due to the induced electric field. This diminishes the width of the depletion layer and leads to an accumulation of electrons in the $\mathrm{n}^{+}$-type part and of holes in the p-type material (Figure 6.6d). These accumulated electrons on the $n^{+}-S i$ side are subsequently used for the regioselective deposition of silver.

We hypothesize that the unmodified band occurs due to the depletion layer of the $\mathrm{n}^{+} / \mathrm{p}$ junction. The depletion layer width $(W)$ of an abrupt $\mathrm{n}^{+} / \mathrm{p}$ junction under equilibrium conditions is given by Equation 6.1 and 6.2:

$$
\begin{aligned}
& W=\sqrt{\frac{2 \varepsilon\left(N_{D}+N_{A}\right) \phi_{b i}}{q N_{D} N_{A}}} \\
& \phi_{b i}=\frac{k_{B} T}{q} \ln \left(\frac{N_{D} N_{A}}{n_{i}^{2}}\right)
\end{aligned}
$$

in which $\varepsilon$ is the permittivity of the material $\left(1.034 \times 10^{-10} \mathrm{AsV}^{-1} \mathrm{~m}^{-1}\right.$ for $\left.\mathrm{Si}\right), N_{D}$ and $N_{A}$ are the donor and acceptor densities $\left(\mathrm{m}^{-3}\right), \phi_{b i}$ is the built-in potential $(\mathrm{V}), \quad q$ is the unit charge $\left(1.602 \times 10^{-19} \mathrm{C}\right), k_{B}$ is Boltzmann's constant $\left(1.3806 \times 10^{-23} \mathrm{JK}^{-1}\right), T$ is the absolute temperature $(\mathrm{K})$ and $n_{i}$ is the intrinsic carrier concentration $\left(1.0 \times 10^{16} \mathrm{~m}^{-3}\right.$ at $300 \mathrm{~K}$ for silicon). The boron doping level, $N_{A}$, of the base wafer of the specimen was determined $\left(1.4 \times 10^{21} \mathrm{~m}^{-3}\right)$ by measuring its sheet resistance. The calculated depletion layer width depends only slightly on the heavily doped material. Based on secondary ion mass spectroscopy (SIMS) (Figure 6.7) data of a phosphorus-doped specimen we used $N_{D}$ doping levels in the range of $10^{22} \mathrm{~m}^{-3}$ to $10^{24} \mathrm{~m}^{-3}$ for calculations. Consequently, the expected depletion layer width in equilibrium ranges from $0.83 \mu \mathrm{m}$ to $0.84 \mu \mathrm{m}$, respectively. Any influences of the silicon/electrolyte junction and changes of the depletion layer width upon an applied reverse bias in the dark or under illumination are not considered, due to the unknown IR drop caused by electron flow from $\mathrm{p}-\mathrm{Si}$ to the solution in the dark and to $\mathrm{n}^{+}-\mathrm{Si}$ under illumination. The values for the width of the depletion layer obtained by this simplified model correspond well with experimental observations of the 
width of the deposition-free zone, for which typically values of $0.9 \mu \mathrm{m}$ (Figure 6.5c) have been found.

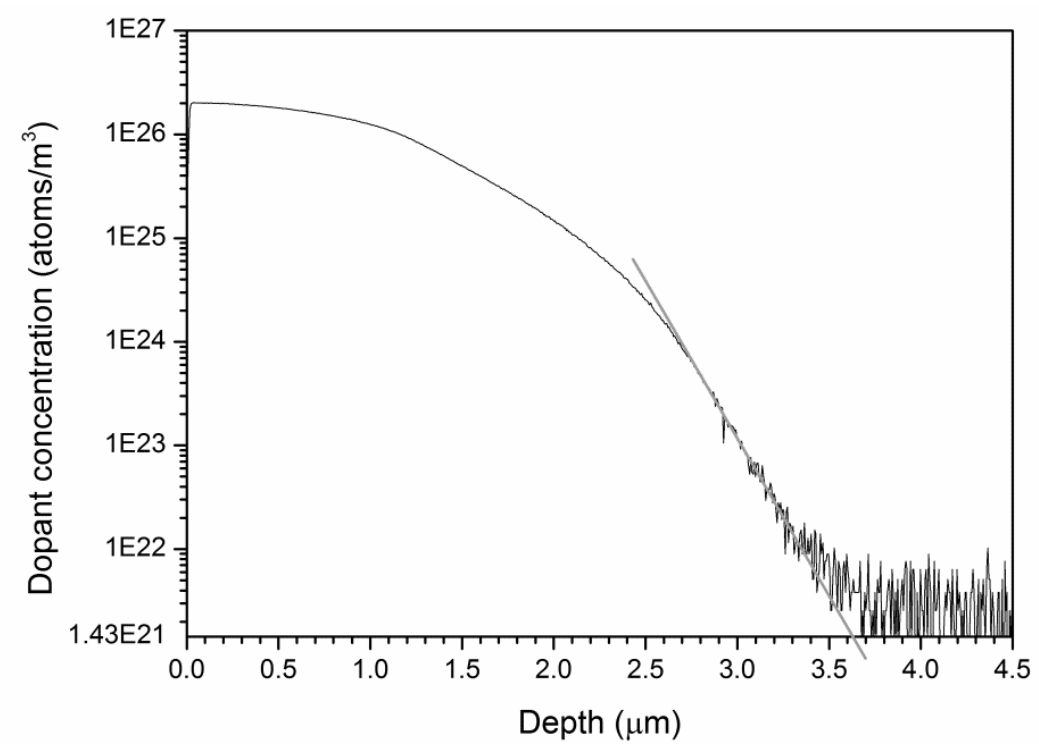

Figure 6.7: Secondary Ion Mass Spectroscopy (SIMS) depth profile of phosphorus dopant concentration in a boron doped silicon specimen with a concentration of $1.43 \times 10^{21}$ atoms $/ \mathrm{m}^{3}$. The junction position is determined by extrapolation of the linear regime towards boron background doping concentration.

According to the theory given above, the depletion layer is expected to penetrate mostly into the low-doped p-region, and therefore the junction should be positioned close to the top of the metal-free zone of the wires. In more quantitative detail, the estimated penetration depth of the depletion layer into the $\mathrm{n}^{+}$-Si side $\left(W_{n}\right)$ is in the range of $0.104 \mu \mathrm{m}$ to $0.001 \mu \mathrm{m}$, while the depths at the $\mathrm{p}$-Si side $\left(W_{p}\right)$ amount to $0.724 \mu \mathrm{m}$ to $0.840 \mu \mathrm{m}$, using $N_{D}$ values of $10^{22} \mathrm{~m}^{-3}$ and $10^{24} \mathrm{~m}^{-3}$, respectively, calculated with Equation 6.3 and 6.4:

$$
\begin{aligned}
& W_{n}=\frac{N_{A} W}{N_{A}+N_{D}} \\
& W_{p}=\frac{N_{D} W}{N_{A}+N_{D}}
\end{aligned}
$$

Based on the observed deposition of $\mathrm{Ag}$ at the top $2.4 \mu \mathrm{m}$ of the wires (Figure 6.5c), the junction is expected to be positioned at about the same height from the top of the microwires. To locate the junction position 
experimentally, we performed SIMS measurements (Figure 6.7), ball grooving and staining of the junction of a doped flat wafer in a $\mathrm{CrO}_{3} / \mathrm{HF}$ aqueous solution (as explained in Chapter 3), and wet etching by the same staining solution of a microwire sample with an axial junction (Figure 6.8). The results are different: SIMS, ball grooving in combination with staining, and a diffusion model for predicting the doping profile at the drive-in settings used here ${ }^{[28]}$ indicate the junction to be located at approximately $3.6 \mu \mathrm{m}$ from the top. In contrast, the doped microwires show a transition at $2.2 \mu \mathrm{m}$ from the top (Figure 6.8). In the latter case, however, a transition becomes visible due to a higher etching rate of $\mathrm{n}^{+}-\mathrm{Si}$ compared to $\mathrm{p}$-Si. Consequently, a small fraction is etched from the microwire top, suggesting the transition being close to the $\mathrm{Ag}$ boundary at $2.4 \mu \mathrm{m}$ from microwire tops. However, this may not necessarily prove exact correspondence with the position of the junction.

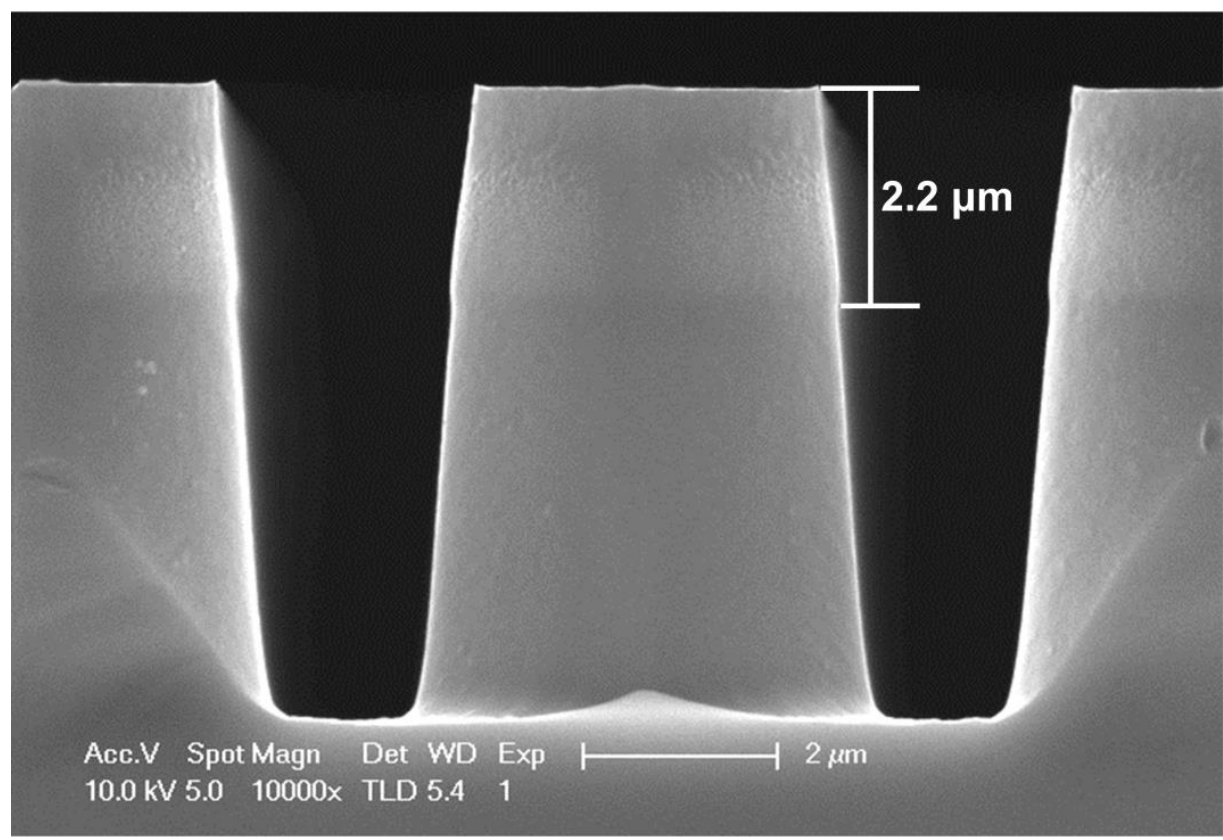

Figure 6.8: Cross-sectional SEM image of silicon microwires with an axial $\mathrm{n}^{+} / \mathrm{p}$ junction stained in $\mathrm{a}_{\mathrm{CrO}_{3}} / \mathrm{HF}$ aqueous solution for $2 \mathrm{~min}$.

The boundary of silver deposition in the dark was observed at the same location as for deposition under illumination, i.e. at $2.4 \mu \mathrm{m}$ from the microwire top (Figure 6.4). The absence of the unmodified band is in this case is 
not clear. We speculate that it might be either due to electronic effects in the microwires or electrostatic attraction or repulsion of precursor metal ions. The formation of a Schottky contact between $\mathrm{Ag}$ and $\mathrm{p}$-Si, of which the depletion layer partially overlaps with the $\mathrm{n}^{+} / \mathrm{p}$ junction, may reduce the $\mathrm{n}^{+} / \mathrm{p}$ junction depletion layer width at the microwires surface, thereby allowing Ag deposition up to the junction in the dark. Furthermore, silver deposition on the depletion layer of the $\mathrm{n}^{+} / \mathrm{p}$ junction may be caused by the attraction of the positively charged $\mathrm{Ag}^{+}$ions in solution to the negatively charged depleted layer in $\mathrm{p}-\mathrm{Si}$ (Figure 6.6b). In contrast, platinum ions are present as negatively charged $\left[\mathrm{PtCl}_{6}\right]^{2-}$ complexes ${ }^{[29]}$ and would be repelled by the depleted layer in $\mathrm{p}-\mathrm{Si}$.

\subsection{Conclusions}

In conclusion, we have developed a new method to functionalize semiconductor wires with an axial junction spatioselectively by utilizing its differences in electronic nature in the dark and under illumination. We have demonstrated separate functionalization of the $\mathrm{n}^{+}$- and $\mathrm{p}$-type segments of silicon microwires, as well as the combined dual functionalization, with electrochemically deposited platinum particles and photoelectrochemically deposited silver particles, respectively. More investigation is needed to understand the observed unmodified band between deposited particles of $\mathrm{Pt}$ in the dark and $\mathrm{Ag}$ under illumination, its absence in the deposition of $\mathrm{Ag}$ in the dark and under illumination, as well as to clarify its origin. However, the finding of this phenomenon may help to improve fundamental understanding of interfaces between semiconductors, metals and electrolytes.

The proposed concept is highly selective, relatively simple and can be potentially also applied to wires or wire arrays with semiconductorsemiconductor axial diode heterojunctions or metal-semiconductor Schottky junctions. Besides reductive electrodeposition of metals, any other reductive or electron-induced reaction, such as electrochemical grafting with organic molecules is potentially possible. Also, oxidative or electron-poor surface reactions are conceivable if an inverted junction is constructed and care is taken to prevent semiconductor surface oxidation. 
Thus, we believe that our method has great potential to improve the ability of creating micro-sized structures with complex compositions and engineer multifunctional devices and systems with new functions.

\section{Acknowledgements}

Alexander Milbrat is greatly acknowledged for the ideas, discussions, figures and electrochemical measurements in this chapter. Roald Tiggelaar and Recep Kas are acknowledged for the discussions. Mark Smithers is thanked for the HR-SEM and EDX imaging. 


\subsection{References}

[1] N. P. Dasgupta, J. Sun, C. Liu, S. Brittman, S. C. Andrews, J. Lim, H. Gao, R. Yan, P. Yang, Adv. Mater., 2014, 26, 2137-2184.

[2] E. L. Warren, H. A. Atwater, N. S. Lewis, J. Phys. Chem. C, 2013, 118, 747-759.

[3] F. Patolsky, C. M. Lieber, Mater. Today, 2005, 8, 20-28.

[4] A. I. Hochbaum, P. Yang, Chem. Rev., 2010, 110, 527-546.

[5] Y. Cui, Q. Wei, H. Park, C. M. Lieber, Science, 2001, 293, 1289-1292.

[6] Y. M. A. Yamada, Y. Yuyama, T. Sato, S. Fujikawa, Y. Uozumi, Angew. Chem., Int. Ed., 2014, 53, 127-131.

[7] M. R. Shaner, K. T. Fountaine, S. Ardo, R. H. Coridan, H. A. Atwater, N. S. Lewis, Energy Environ. Sci., 2014, 7, 779-790.

[8] M. Y. Bashouti, K. Sardashti, S. W. Schmitt, M. Pietsch, J. Ristein, H. Haick, S. H. Christiansen, Prog. Surf. Sci., 2013, 88, 39-60.

[9] N. Gao, W. Zhou, X. Jiang, G. Hong, T.-M. Fu, C. M. Lieber, Nano Lett., 2015, 15, $2143-$ 2148.

[10] A. Walther, A. H. E. Müller, Chem. Rev., 2013, 113, 5194-5261.

[11] Z. Jiang, Q. Qing, P. Xie, R. Gao, C. M. Lieber, Nano Lett., 2012, 12, 1711-1716.

[12] A. M. Ionescu, H. Riel, Nature, 2011, 479, 329-337.

[13] C.-Y. Wen, M. C. Reuter, J. Bruley, J. Tersoff, S. Kodambaka, E. A. Stach, F. M. Ross, Science, 2009, 326, 1247-1250.

[14] A. L. Vallett, S. Minassian, P. Kaszuba, S. Datta, J. M. Redwing, T. S. Mayer, Nano Lett., 2010, 10, 4813-4818.

[15] L. Guo, G. Oskam, A. Radisic, P. M. Hoffmann, P. C. Searson, J. Phys. D: Appl. Phys., 2011, 44, 443001.

[16] M. Hocevar, G. Immink, M. Verheijen, N. Akopian, V. Zwiller, L. Kouwenhoven, E. Bakkers, Nat. Commun., 2012, 3, 1266.

[17] J. Motohisa, Y. Kohashi, S. Maeda, Nano Lett., 2014, 14, 3653-3660.

[18] B. Tian, T. J. Kempa, C. M. Lieber, Chem. Soc. Rev., 2009, 38, 16-24.

[19] M. Yao, N. Huang, S. Cong, C.-Y. Chi, M. A. Seyedi, Y.-T. Lin, Y. Cao, M. L. Povinelli, P. D. Dapkus, C. Zhou, Nano Lett., 2014, 14, 3293-3303.

[20] J. D. Christesen, X. Zhang, C. W. Pinion, T. A. Celano, C. J. Flynn, J. F. Cahoon, Nano Lett., 2012, 12, 6024-6029.

[21] M. S. Gudiksen, L. J. Lauhon, J. Wang, D. C. Smith, C. M. Lieber, Nature, 2002, 415, 617-620.

[22] C. Liu, J. Tang, H. M. Chen, B. Liu, P. Yang, Nano Lett., 2013, 13, 2989-2992.

[23] S. W. Boettcher, E. L. Warren, M. C. Putnam, E. A. Santori, D. Turner-Evans, M. D. Kelzenberg, M. G. Walter, J. R. McKone, B. S. Brunschwig, H. A. Atwater, N. S. Lewis, J. Am. Chem. Soc., 2011, 133, 1216-1219.

[24] Y. Qu, L. Liao, R. Cheng, Y. Wang, Y.-C. Lin, Y. Huang, X. Duan, Nano Lett., 2010, 10, 1941-1949.

[25] T. Wang, H. Chen, K. Liu, S. Wang, P. Xue, Y. Yu, P. Ge, J. Zhang, B. Yang, ACS Appl. Mater. Interfaces, 2015, 7, 376-382.

[26] T. Wang, H. Chen, K. Liu, Y. Li, P. Xue, Y. Yu, S. Wang, J. Zhang, E. Kumacheva, B. Yang, Nanoscale, 2014, 6, 3846-3853.

[27] L. Mammen, K. Bley, P. Papadopoulos, F. Schellenberger, N. Encinas, H.-J. Butt, C. K. Weiss, D. Vollmer, Soft Matter, 2015, 11, 506-515.

[28] M. Uematsu, J. Appl. Phys., 1997, 82, 2228-2246.

[29] C. R. K. Rao, D. C. Trivedi, Coord. Chem. Rev., 2005, 249, 613-631.

[30] Y. L. Kawamura, T. Sakka, Y. H. Ogata, J. Electrochem. Soc., 2005, 152, C701-C705. 


\section{Summary and Outlook}

Silicon is a widely used material in the photovoltaic industry, and its advantageous properties and availability ensure that it can play an important role in the transition to a sustainable production of energy. Apart from already commercially available PV cells, silicon can also take part in new alternatives, such as solar-to-fuel (S2F) devices. However, before silicon can be used in such a device, it has to be altered to withstand different conditions, for example a water environment in case of a water splitting S2F device. Moreover, the structuring of silicon, e.g. into micropillar arrays, provides ways to enhance the performance of both PV and S2F devices. This thesis discusses the use of silicon as a base material for the structuring of solar cells, and their optimization and modification.

Chapter 3 has reported the fabrication and doping of silicon micropillars, followed by an electrical characterization. The micropillars were made by means of standard photolithography and deep reactive ion etching, with a diameter of $4 \mu \mathrm{m}$. The radial $\mathrm{p} / \mathrm{n}$ junctions were formed by different methods, such as low-pressure chemical vapor deposition of phosphorus and solid source dotation of boron. It was verified that the junction was formed homogeneously over the full height of the pillar as made visible by focused ion beam cutting, staining, and SEM imaging. In both phosphorus and boron-doped samples, the pillared solar cells showed an improvement in efficiency, compared to flat solar cells.

The micropillars were further optimized, as described in Chapter 4, where the height and junction depth were varied to determine their optimal values. The height of the pillars was varied between 0 and $60 \mu \mathrm{m}$, and showed the highest solar cell efficiency for a height of $40 \mu \mathrm{m}$. The efficiency of higher pillars was lower, probably due to the fact that etching of the silicon introduced more defect surface states, leading to more recombination. The optimum for the junction depth was found to be $790 \mathrm{~nm}$. Samples with smaller depths were not functional, as the diode behavior showed leakage. For junction depths 
$>790 \mathrm{~nm}$, the efficiency dropped, which was explained by the narrowing of the undoped inner part of the pillars by the depletion zone.

Chapter 5 has discussed the further optimization of silicon pillar devices, by means of a passivation and anti-reflective coating. The defects introduced in the silicon by deep reactive ion etching could be eliminated by applying a thin layer of either $\mathrm{Al}_{2} \mathrm{O}_{3}$ (by atomic layer deposition), $\mathrm{SiN}_{\mathrm{x}}$ or $\mathrm{SiO}_{2}$ (both by low-pressure chemical vapor deposition). By tuning the thickness of this layer, the layer also acted as an anti-reflective coating, which increased the current density of all samples. The 3D and conformal deposition of these layers was also investigated by focused ion beam and SEM imaging. For both $p$ - and n-type doped samples, atomic layer deposition of $\mathrm{Al}_{2} \mathrm{O}_{3}$ gave the highest increase in solar cell efficiency.

Finally, in Chapter 6, a method for the functionalization of silicon micropillars was discussed. For that purpose, axial $p / n$ junctions were fabricated by spin-on-dopant diffusion, followed by the cryogenic etching of silicon, which minimized sidewall damaging. By using the axial nature of the junction, the bottom and top parts were functionalized selectivity with platinum (in the dark) and silver (under illumination), respectively. The metals were deposited by electrodeposition, without the use of a masking step. A non-functionalized zone between the $\mathrm{n}$ - and $\mathrm{p}$-type parts was observed, and calculations suggested that this matches the depletion zone.

To conclude, silicon micropillars with radial $\mathrm{p} / \mathrm{n}$ junctions were fabricated and optimized using various techniques. These structures might also be applied in silicon-based S2F devices, to enhance the light absorption, ultimately increasing the overall efficiency of such a device. The jointly passivating and anti-reflective coating could even function as a chemically protective layer, to increase the stability of silicon in an aqueous environment. In addition, it was shown that (axially doped) silicon pillars could be functionalized with different materials, by using the $\mathrm{p} / \mathrm{n}$ junction and its electrical performance in dark and under illumination to direct the flow of electrons. This approach could be useful to drive reactions that require different catalysts on the same pillar. 


\section{Samenvatting en Visie}

Silicium is een veelgebruikt materiaal in de zonnecelindustrie en, vanwege de uitstekende eigenschappen en grote beschikbaarheid, zal het een belangrijke rol kunnen spelen bij de transitie naar een duurzame energiewinning. Behalve voor zonnecellen kan het ook belangrijk zijn voor nieuwe alternatieven, zoals een zonlicht-tot-brandstof (S2F-)apparaat. Voordat silicium daarvoor gebruikt kan worden, moet het eerst gemodificeerd worden zodat het onder verschillende condities kan functioneren, zoals in een waterige oplossing die nodig is bij watersplitsing. Bovendien kan het structureren van silicium een grote bijdrage leveren aan de prestaties van apparaten die zonne-energie omzetten. In dit proefschrift wordt het gebruik van silicium als basismateriaal voor het structureren van zonnecellen beschreven, alsmede de optimalisatie en modificatie van deze materialen.

In Hoofdstuk 3 is de fabricage en dotering van silicium micropilaren beschreven, gevolgd door een elektrische karakterisatie. De micropilaren zijn gemaakt met behulp van standaard-fotolithografie en droog-etsen (DRIE), en hebben een diameter van $4 \mu \mathrm{m}$. De radiale junctie is gevormd door middel van verschillende doteringstechnieken, zoals de depositie van fosfor bij lage druk (LPCVD) en de dotering van boor vanuit een vaste bron (SSD). Het is aangetoond dat de radiale junctie homogeen door de pilaren gevormd was door een dwarsdoorsnede te bekijken van een pilaar nadat deze doorgesneden was met behulp een gefocusseerde ionenbundel (FIB). De elektrische eigenschappen van zowel fosfor- als boor-gedoteerde pilaren waren verbeterd in vergelijking met vlakke zonnecellen.

De hiervoor beschreven micropilaren zijn verder geoptimaliseerd, zoals beschreven in Hoofdstuk 4, door middel van de variatie van de hoogte en de junctiediepte. De hoogte is gevariëerd tussen de 0 en $60 \mu \mathrm{m}$, en gaf de hoogste efficiëntie bij een hoogte van $40 \mu \mathrm{m}$. De lagere efficiëntie van hogere pilaren is toegeschreven aan het toenemend aantal oppervlaktedefecten bij 
toenemende pilaarhoogte, hetgeen tot meer recombinatie leidt. Voor de junctie-diepte is een optimum van $790 \mathrm{~nm}$ gevonden. Pilaren met dunnere juncties gaven geen correct werkende diode en vertoonden lekkage in de elektrische metingen. Junctiedieptes groter dan $790 \mathrm{~nm}$ lieten een lagere efficiëntie zien, hetgeen verklaard is door het sluiten van de junctie omdat de depletiezones elkaar raken in het binnenste van de pilaren.

In Hoofdstuk 5 is een verdere optimalisatie besproken, waarbij een laag gebruikt is om passivatie en anti-reflectiviteit te bewerkstelligen. De defecten die geïntroduceerd zijn door het etsen, konden worden verminderd door een dunne laag $\mathrm{Al}_{2} \mathrm{O}_{3}$ (door middel van atoomlaag-depositie, ALD), $\mathrm{SiN}_{\mathrm{x}}$ of $\mathrm{SiO}_{2}$ (beide door middel van LPCVD) op te brengen. Door de dikte van de laag goed af te stemmen op de lichtabsorptie, kon de laag tevens als een antireflectielaag gebruikt worden. Het $3 \mathrm{D}$ deponeren van deze lagen is onderzocht, wederom met behulp van FIB. Voor zowel de $\mathrm{p}$ - als n-gedoteerde zonnecellen bleek een laag van $\mathrm{Al}_{2} \mathrm{O}_{3}$ de grootste efficiëntieverhoging te geven.

In Hoofdstuk 6 tenslotte is een methode besproken voor het functionaliseren van silicium. Hiervoor is een axiale junctie in vlak silicium gemaakt met behulp van 'spin-on-doping', gevolgd door cryogeen etsen om zo micropilaren te verkrijgen die deels $p$ - en deels $n$-gedoopt zijn. Door gebruik te maken van de $\mathrm{p} / \mathrm{n}$ junctie konden de onderste en bovenste helften apart gefunctionaliseerd worden met respectievelijk platina (in het donker) en zilver (in het licht). Deze metalen zijn afgezet door middel van elektrochemische depositie, zonder daarbij gebruik te maken van een maskerlaag. Een klein, niet-gefunctionaliseerd pilaargedeelte is waargenomen tussen de delen die met platina en zilver bedekt waren en berekeningen geven aan dat dit overeenkomt met de breedte van de depletiezone.

Samenvattend blijkt dat micropilaren met radiale juncties goed gefabriceerd zijn en verder geoptimaliseerd hebben kunnen worden, door gebruik te maken van verschillende technieken. Deze siliciumstructuren kunnen wellicht gebruikt 
worden voor S2F-apparaten, om zo zoveel mogelijk licht te absorberen en daarmee de efficiëntie te verhogen. De passivatie- en anti-reflectielaag kan wellicht ook als chemische beschermlaag dienen, om zo de stabiliteit van de zonnecellen te verbeteren in waterige oplossingen. Bovendien is aangetoond dat axiaal gedoteerde siliciumpilaren met verschillende materialen gefunctionaliseerd konden worden door gebruik te maken van de eigenschappen van de $\mathrm{p} / \mathrm{n}$-junctie in licht en donker. Deze aanpak kan een bijdrage leveren aan het aanbrengen en gebruiken van verschillende katalysatoren op dezelfde pilaren. 


\section{Appendix A}

\section{A.1. Process flow radial junctions}

Process parameters for radial junctions in micropillars using solid source dotation of boron or low-pressure chemical vapor deposition of phosphorus.

\section{A.1.1. Wafer selection}

\begin{tabular}{|ll}
\hline $\begin{array}{l}\text { Substrate Silicon }<100>\text { OSP } \\
\text { N-type }\end{array}$ & Orientation: $<100>$ \\
(\#subs109) & Diameter: $100 \mathrm{~mm}$ \\
& Thickness: $381 \mu \mathrm{m} \pm 15$ \\
& Polished: Single side polished \\
& Resistivity: $1-10 \Omega \mathrm{cm}$ \\
& Type: $\mathrm{N}$
\end{tabular}

OR

\begin{tabular}{|ll}
\hline Substrate Silicon $<100>$ OSP & Orientation: $<100>$ \\
P-type & Diameter: $100 \mathrm{~mm}$ \\
(\#subs108) & Thickness: $525 \mu \mathrm{m} \pm 25$ \\
& Polished: Single side polished \\
& Resistivity: $5-10 \Omega \mathrm{cm}$ \\
& Type: $P$
\end{tabular}

\section{A.1.2. Wafer processing}

Steps 1-31 are only necessary in the process of $n$-type wafers, p-type wafers start at 32.

\begin{tabular}{|c|c|c|c|}
\hline \multicolumn{2}{|r|}{ Process } & & \multirow{2}{*}{$\begin{array}{l}\text { Comment } \\
\text { N-TYPE } \\
\text { ONLY BEGIN } \\
\end{array}$} \\
\hline 1 & $\begin{array}{l}\text { Cleaning in } 99 \% \mathrm{HNO}_{3} \\
\text { (\#clean001) }\end{array}$ & $\begin{array}{l}\text { NL-CLR-WB14 } \\
\text { Purpose: removal of organic traces. } \\
\text { - Beaker } 1: 99 \% \mathrm{HNO}_{3} \\
\text { - Time: } 5 \text { min }\end{array}$ & \\
\hline 2 & $\begin{array}{l}\text { Cleaning in } 99 \% \mathrm{HNO}_{3} \\
\text { (\#clean001) }\end{array}$ & $\begin{array}{l}\text { NL-CLR-WB14 } \\
\text { Purpose: removal of organic traces. } \\
\text { - Beaker 2: } 99 \% \mathrm{HNO}_{3} \\
\text { - Time: } 5 \mathrm{~min}\end{array}$ & \\
\hline 3 & $\begin{array}{l}\text { Quick Dump Rinse } \\
\text { (QDR) } \\
\text { (\#rinse119) }\end{array}$ & $\begin{array}{l}\text { NL-CLR- WB14 } \\
\text { Purpose: removal of traces of chemical agents. }\end{array}$ & \\
\hline 4 & $\begin{array}{l}\text { Cleaning in } 69 \% \mathrm{HNO}_{3} \\
\text { at } 95{ }^{\circ} \mathrm{C} \\
(\# \text { clean003) }\end{array}$ & $\begin{array}{l}\text { NL-CR-WB14 } \\
\text { Purpose: removal of metallic traces. } \\
\text { - Beaker } 3 \mathrm{a} \text { or beaker } 3 \mathrm{~b}: 69 \% \mathrm{HNO}_{3} \\
\text { - Temp: } 95^{\circ} \mathrm{C} \\
\text { - Time: } 10 \mathrm{~min}\end{array}$ & \\
\hline 5 & $\begin{array}{l}\text { Quick Dump Rinse } \\
\text { (QDR) } \\
\text { (\#rinse119) }\end{array}$ & $\begin{array}{l}\text { NL-CLR- WB14 } \\
\text { Purpose: removal of traces of chemical agents. }\end{array}$ & \\
\hline
\end{tabular}




\begin{tabular}{|c|c|c|c|}
\hline 6 & $\begin{array}{l}\text { Substrate drying } \\
\text { (WB14) } \\
\text { (\#dry159) }\end{array}$ & $\begin{array}{l}\text { NL-CLR-WB14 } \\
\text { Batch drying of substrates }\end{array}$ & \\
\hline 7 & $\begin{array}{l}\text { Etching in HF (1\%) } \\
\text { (\#etch127) }\end{array}$ & $\begin{array}{l}\text { NL-CLR-WB15 } \\
\text { Purpose: removal of native } \mathrm{SiO}_{2} \text { from silicon wafers. } \\
\text { Beaker: } \mathrm{HF} 1 \% \\
\text { Time: } 1 \text { min }\end{array}$ & \\
\hline 8 & $\begin{array}{l}\text { Quick Dump Rinse } \\
\text { (QDR) } \\
\text { (\#rinse119) }\end{array}$ & $\begin{array}{l}\text { NL-CLR- WB15 } \\
\text { Purpose: removal of traces of chemical agents. }\end{array}$ & \\
\hline 9 & $\begin{array}{l}\text { Substrate drying } \\
\text { (WB15) } \\
\text { (\#dry160) }\end{array}$ & $\begin{array}{l}\text { NL-CLR-WB15 } \\
\text { Batch drying of substrates }\end{array}$ & \\
\hline 10 & $\begin{array}{l}\text { LPCVD of SiRN }(250 \\
\text { MPa) } \\
(\# \text { film155) }\end{array}$ & $\begin{array}{l}\text { NL-CLR-Tempress LPCVD G4 } \\
\text { Program: N2 LSNIT-G4 ( } 200 \text { mTorr) } \\
\text { - } \mathrm{SiH}_{2} \mathrm{Cl}_{2} \text { flow: } 150 \mathrm{sccm} \\
\text { - } \mathrm{NH}_{3} \text { flow: } 50 \mathrm{sccm}\end{array}$ & $\begin{array}{l}100 \mathrm{~nm} \text { in } 12 \\
\min \end{array}$ \\
\hline 11 & $\begin{array}{l}\text { Inspection of } \\
\text { LPCVD/PECVD layers } \\
\text { (\#metro113) }\end{array}$ & $\begin{array}{l}\text { NL-CLR-cold light source } \\
\text { Particle and haze inspection by using cold light source }\end{array}$ & \\
\hline 12 & $\begin{array}{l}\text { Ellipsometer } \\
\text { measurement } \\
\text { (\#metro107) }\end{array}$ & NL-CLR-Woollam M-2000 ellipsometer & \\
\hline 13 & $\begin{array}{l}\text { DRIE of } \mathrm{SiO}_{2}, \text { SiRN } \\
\text { (\#etch157) }\end{array}$ & $\begin{array}{l}\text { NL-CLR- AdixenDE } \\
\text { Layer thickness: }<5 \mu \mathrm{m} \mathrm{SiO}_{2} \\
\text { SH Temp: }-10^{\circ} \mathrm{C}-\text { Pos: } 120 \mathrm{~mm}-\mathrm{He} \text { pres.: } 10 \mathrm{mbar} \\
\text { Flows } \mathrm{C}_{4} \mathrm{~F}_{8}: 20 \mathrm{sccm}-\mathrm{CH}_{4}: 15 \mathrm{sccm}-\mathrm{He}: 150 \mathrm{sccm} \\
\text { Pressure: } 8.510^{-3} \mathrm{mbar} \\
\text { ICP: } 2800 \text { Watt } \\
\text { CCP: } 350 \text { Watt (RF) }\end{array}$ & $\begin{array}{l}\text { Backside only, } \\
45 \mathrm{sec}\end{array}$ \\
\hline 14 & $\begin{array}{l}\text { Chamber clean } \\
\text { AdixenDE } \\
\text { (\#etch201) }\end{array}$ & $\begin{array}{l}\text { NL-CLR-AdixenDE } \\
\text { Chamber clean to remove fluorocarbon } \\
\text { Needed after every } 20 \text { min processing } \\
\text { SH Temp: any- Pos: } 150 \mathrm{~mm} \text { - He pres.: } 10 \text { mbar } \\
\text { Flow } \mathrm{O}_{2}: 200 \text { sccm } \\
\text { APC } 100 \% \text { / Pressure: } 1.510^{-2}-6.0^{*} 10^{-6} \mathrm{mbar} \\
\text { ICP: } 3000 \text { Watt } \\
\text { CCP: } 50 \text { Watt (RF) } \\
\text { Clean with a plain silicon wafer in the etch tool } \\
\text { Cleaning time: } 30 \text { minutes }\end{array}$ & \\
\hline 15 & $\begin{array}{l}\text { Stripping of } \\
\text { photoresist and FC } \\
\text { after DRIE } \\
\text { (\#strip121) }\end{array}$ & $\begin{array}{l}\text { NL-CLR-TePla } 360 \mathrm{M} \\
\text { Recipe } 035\end{array}$ & \\
\hline 16 & $\begin{array}{l}\text { Cleaning in } 99 \% \mathrm{HNO}_{3} \\
\text { (\#clean001) }\end{array}$ & $\begin{array}{l}\text { NL-CLR-WB14 } \\
\text { Purpose: removal of organic traces. } \\
\text { - Beaker 1: } 99 \% \mathrm{HNO}_{3} \\
\text { - Time: } 5 \text { min }\end{array}$ & \\
\hline 17 & $\begin{array}{l}\text { Cleaning in } 99 \% \mathrm{HNO}_{3} \\
\text { (\#clean001) }\end{array}$ & $\begin{array}{l}\text { NL-CLR-WB14 } \\
\text { Purpose: removal of organic traces. } \\
\text { - Beaker 2: } 99 \% \mathrm{HNO}_{3} \\
\text { - Time: } 5 \text { min }\end{array}$ & \\
\hline 18 & $\begin{array}{l}\text { Quick Dump Rinse } \\
\text { (QDR) } \\
\text { (\#rinse119) }\end{array}$ & $\begin{array}{l}\text { NL-CLR-WB14 } \\
\text { Purpose: removal of traces of chemical agents. }\end{array}$ & \\
\hline 19 & $\begin{array}{l}\text { Cleaning in } 69 \% \mathrm{HNO}_{3} \\
\text { at } 95{ }^{\circ} \mathrm{C}\end{array}$ & $\begin{array}{l}\text { NL-CR-WB14 } \\
\text { Purpose: removal of metallic traces. }\end{array}$ & \\
\hline
\end{tabular}




\begin{tabular}{|c|c|c|c|}
\hline & (\#clean003) & $\begin{array}{l}\text { - Beaker } 3 \mathrm{a} \text { or beaker } 3 \mathrm{~b}: 69 \% \mathrm{HNO}_{3} \\
\text { - Temp.: } 95{ }^{\circ} \mathrm{C} \\
\text { - Time: } 10 \mathrm{~min}\end{array}$ & \\
\hline 20 & $\begin{array}{l}\text { Quick Dump Rinse } \\
\text { (QDR) } \\
\text { (\#rinse119) }\end{array}$ & $\begin{array}{l}\text { NL-CLR-WB14 } \\
\text { Purpose: removal of traces of chemical agents. }\end{array}$ & \\
\hline 21 & $\begin{array}{l}\text { Substrate drying } \\
\text { (WB14) } \\
\text { (\#dry159) }\end{array}$ & $\begin{array}{l}\text { NL-CLR-WB14 } \\
\text { Batch drying of substrates }\end{array}$ & \\
\hline 22 & $\begin{array}{l}\text { Etching in HF (1\%) } \\
\text { (\#etch127) }\end{array}$ & $\begin{array}{l}\text { NL-CLR-WB15 } \\
\text { Purpose: removal of native } \mathrm{SiO}_{2} \text { from silicon wafers. } \\
\text { Beaker: } \mathrm{HF} 1 \% \\
\text { Time: } 1 \text { min }\end{array}$ & \\
\hline 23 & $\begin{array}{l}\text { Quick Dump Rinse } \\
\text { (QDR) } \\
\text { (\#rinse119) }\end{array}$ & $\begin{array}{l}\text { NL-CLR-WB15 } \\
\text { Purpose: removal of traces of chemical agents. }\end{array}$ & \\
\hline 24 & $\begin{array}{l}\text { Substrate drying } \\
\text { (WB15) } \\
\text { (\#dry160) }\end{array}$ & $\begin{array}{l}\text { NL-CLR-WB15 } \\
\text { Batch drying of substrates }\end{array}$ & \\
\hline 25 & $\begin{array}{l}\text { LPCVD of PSG (TEOS) } \\
\text { (\#film188) }\end{array}$ & $\begin{array}{l}\text { NL-CLR-LPCVD H4 } \\
\text { - Program: N2-PSG } \\
\text { - } 330 \mathrm{sccm}^{\mathrm{PH}} \mathrm{H}_{3} \\
\cdot 50 \mathrm{sccm} \text { TEOS } \\
\text { - } 45 \text { min deposition }\end{array}$ & \\
\hline 26 & $\begin{array}{l}\text { Anneal at } 1050^{\circ} \mathrm{C} \\
(\# \text { film168) }\end{array}$ & $\begin{array}{l}\text { NL-CLR-Furnace B3 } \\
\text { - Standby temperature: } 800{ }^{\circ} \mathrm{C} \\
\text { - Temp.: } 1050{ }^{\circ} \mathrm{C} \\
\text { - Gas: } \mathrm{N}_{2} \\
\text { - Flow: } 2 \mathrm{~L} / \mathrm{min}\end{array}$ & \\
\hline 27 & $\begin{array}{l}\text { Etching in BHF (1:7) } \\
\text { (\#etch124) }\end{array}$ & $\begin{array}{l}\text { NL-CLR-WB06 } \\
\text { Use dedicated beaker BHF }(1: 7)\end{array}$ & $\begin{array}{l}15 \text { min // Until } \\
\text { hydrophobic }\end{array}$ \\
\hline 28 & $\begin{array}{l}\text { Quick Dump Rinse } \\
\text { (QDR) } \\
\text { (\#rinse119) }\end{array}$ & $\begin{array}{l}\text { NL-CLR-WB06 } \\
\text { Purpose: removal of traces of chemical agents. }\end{array}$ & \\
\hline 29 & $\begin{array}{l}\text { Substrate drying } \\
\text { (\#dry120) }\end{array}$ & $\begin{array}{l}\text { NL-CLR-WB06-08 } \\
\text { Batch drying of substrates }\end{array}$ & \\
\hline 30 & $\begin{array}{l}\text { DRIE of } \mathrm{SiO}_{2}, \text { SiRN } \\
\text { (\#etch157) }\end{array}$ & $\begin{array}{l}\text { NL-CLR- AdixenDE } \\
\text { Layer thickness: < } 5 \mu \mathrm{m} \text { SiO2 } \\
\text { SH Temp: }-10^{\circ} \mathrm{C}-\mathrm{Pos}: 120 \mathrm{~mm}-\mathrm{He} \text { pres.: } 10 \mathrm{mbar} \\
\text { Flows } \mathrm{C}_{4} \mathrm{~F}_{8}: 20 \mathrm{sccm}-\mathrm{CH}_{4}: 15 \mathrm{sccm}-\mathrm{He}: 150 \mathrm{sccm} \\
\text { Pressure: } 8.510^{-3} \mathrm{mbar} \\
\text { ICP: } 2800 \text { Watt } \\
\text { CCP: } 350 \text { Watt (RF) }\end{array}$ & $\begin{array}{l}\text { Backside only, } \\
45 \mathrm{sec}\end{array}$ \\
\hline 31 & $\begin{array}{l}\text { Chamber clean Adixen } \\
\text { DE } \\
\text { (\#etch201) }\end{array}$ & $\begin{array}{l}\text { NL-CLR-AdixenDE } \\
\text { Chamber clean to remove fluorocarbon } \\
\text { Needed after every } 20 \text { min processing } \\
\text { SH Temp: any- Pos: } 150 \mathrm{~mm} \text { - He pres.: } 10 \mathrm{mbar} \\
\text { Flow } \mathrm{O}_{2}: 200 \text { sccm } \\
\text { APC } 100 \% \text { / Pressure: } 1.510^{-2-} 6.0^{*} 10^{-6} \mathrm{mbar} \\
\text { ICP: } 3000 \text { Watt } \\
\text { CCP: } 50 \text { Watt (RF) }\end{array}$ & $\begin{array}{l}\text { N-TYPE } \\
\text { ONLY END }\end{array}$ \\
\hline 32 & $\begin{array}{l}\text { Cleaning in } 99 \% \mathrm{HNO}_{3} \\
\text { (\#clean001) }\end{array}$ & $\begin{array}{l}\text { NL-CLR-WB14 } \\
\text { Purpose: removal of organic traces. } \\
\text { - Beaker } 1: 99 \% \mathrm{HNO}_{3} \\
\text { - Time: } 5 \text { min }\end{array}$ & \\
\hline
\end{tabular}




\begin{tabular}{|c|c|c|c|}
\hline 33 & $\begin{array}{l}\text { Cleaning in } 99 \% \mathrm{HNO}_{3} \\
\text { (\#clean001) }\end{array}$ & $\begin{array}{l}\text { NL-CLR-WB14 } \\
\text { Purpose: removal of organic traces. } \\
\text { - Beaker 2: } 99 \% \mathrm{HNO}_{3} \\
\text { - Time: } 5 \text { min }\end{array}$ & \\
\hline 34 & $\begin{array}{l}\text { Quick Dump Rinse } \\
\text { (QDR) } \\
\text { (\#rinse119) }\end{array}$ & $\begin{array}{l}\text { NL-CLR-WB14 } \\
\text { Purpose: removal of traces of chemical agents. }\end{array}$ & \\
\hline 35 & $\begin{array}{l}\text { Cleaning in } 69 \% \mathrm{HNO}_{3} \\
\text { at } 95{ }^{\circ} \mathrm{C} \\
(\# \text { clean003) }\end{array}$ & $\begin{array}{l}\text { NL-CR-WB14 } \\
\text { Purpose: removal of metallic traces. } \\
\text { - Beaker } 3 \text { a or beaker } 3 \mathrm{~b}: 69 \% \mathrm{HNO}_{3} \\
\text { - Temp.: } 95^{\circ} \mathrm{C} \\
\text { - Time: } 10 \text { min }\end{array}$ & \\
\hline 36 & $\begin{array}{l}\text { Quick Dump Rinse } \\
\text { (QDR) } \\
\text { (\#rinse119) }\end{array}$ & $\begin{array}{l}\text { NL-CLR-WB14 } \\
\text { Purpose: removal of traces of chemical agents. }\end{array}$ & \\
\hline 37 & $\begin{array}{l}\text { Substrate drying } \\
\text { (WB14) } \\
\text { (\#dry159) }\end{array}$ & $\begin{array}{l}\text { NL-CLR-WB14 } \\
\text { Batch drying of substrates }\end{array}$ & \\
\hline 38 & $\begin{array}{l}\text { Etching in HF (1\%) } \\
\text { (\#etch127) }\end{array}$ & $\begin{array}{l}\text { NL-CLR-WB15 } \\
\text { Purpose: removal of native } \mathrm{SiO}_{2} \text { from silicon wafers. } \\
\text { Beaker: } \mathrm{HF} 1 \% \\
\text { Time: } 1 \text { min }\end{array}$ & \\
\hline 39 & $\begin{array}{l}\text { Quick Dump Rinse } \\
\text { (QDR) } \\
\text { (\#rinse119) }\end{array}$ & $\begin{array}{l}\text { NL-CLR-WB15 } \\
\text { Purpose: removal of traces of chemical agents. }\end{array}$ & \\
\hline 40 & $\begin{array}{l}\text { Substrate drying } \\
\text { (WB15) } \\
\text { (\#dry160) }\end{array}$ & $\begin{array}{l}\text { NL-CLR-WB15 } \\
\text { Batch drying of substrates }\end{array}$ & \\
\hline 41 & $\begin{array}{l}\text { LPCVD of SiRN }(250 \\
\text { MPa) } \\
(\# f i l m 155)\end{array}$ & $\begin{array}{l}\text { NL-CLR-Tempress LPCVD G4 } \\
\text { Program: N2 LSNIT-G4 (200 mTorr) } \\
\text { - } \mathrm{SiH}_{2} \mathrm{Cl}_{2} \text { flow: } 150 \mathrm{sccm} \\
\text { - } \mathrm{NH}_{3} \text { flow: } 50 \mathrm{sccm}\end{array}$ & $\begin{array}{l}100 \mathrm{~nm} \text { in } 12 \\
\min \end{array}$ \\
\hline 42 & $\begin{array}{l}\text { Inspection of } \\
\text { LPCVD/PECVD layers } \\
\text { (\#metro113) }\end{array}$ & $\begin{array}{l}\text { NL-CLR-cold light source } \\
\text { Particle and haze inspection by using cold light source } \\
\text { Inspection of LPCVD/PECVD deposited layers like: } \\
\mathrm{TEOS} \mathrm{SiO}_{2}, \mathrm{Si}_{3} \mathrm{~N}_{4}, \mathrm{SiRN}, \text { Poly-Silicon } \\
\text { Procedure: use streaking light for inspection }\end{array}$ & \\
\hline 43 & $\begin{array}{l}\text { Ellipsometer } \\
\text { measurement } \\
\text { (\#metro107) }\end{array}$ & NL-CLR-Woollam M-2000 ellipsometer & \\
\hline 44 & $\begin{array}{l}\text { DRIE of } \mathrm{SiO}_{2}, \mathrm{SiRN} \\
\text { (\#etch157) }\end{array}$ & $\begin{array}{l}\text { NL-CLR- AdixenDE } \\
\text { Layer thickness: }<5 \mu \mathrm{m} \text { SiO2 } \\
\text { SH Temp: }-10^{\circ} \mathrm{C}-\mathrm{Pos}: 120 \mathrm{~mm}-\mathrm{He} \text { pres.: } 10 \mathrm{mbar} \\
\text { Flows } \mathrm{C}_{4} \mathrm{~F}_{8}: 20 \mathrm{sccm}-\mathrm{CH}_{4}: 15 \mathrm{sccm}-\mathrm{He}: 150 \mathrm{sccm} \\
\text { Pressure: } 8.510^{-3} \mathrm{mbar} \\
\text { ICP: } 2800 \text { Watt } \\
\text { CCP: } 350 \text { Watt (RF) }\end{array}$ & $\begin{array}{l}\text { Front side } \\
\text { only }\end{array}$ \\
\hline 45 & $\begin{array}{l}\text { Chamber clean Adixen } \\
\text { DE } \\
\text { (\#etch201) }\end{array}$ & $\begin{array}{l}\text { NL-CLR-AdixenDE } \\
\text { Chamber clean to remove fluorocarbon } \\
\text { Needed after every } 20 \text { min processing } \\
\text { SH Temp: any- Pos: } 150 \mathrm{~mm} \text { - He pres.: } 10 \text { mbar } \\
\text { Flow } \mathrm{O}_{2}: 200 \text { sccm } \\
\text { APC } 100 \% \text { / Pressure: } 1.510^{-2}-6.0^{*} 10^{-6} \mathrm{mbar} \\
\text { ICP: } 3000 \text { Watt } \\
\text { CCP: } 50 \text { Watt (RF) } \\
\text { Clean with a plain silicon wafer in the etch tool } \\
\text { Cleaning time: } 30 \text { minutes }\end{array}$ & \\
\hline
\end{tabular}




\begin{tabular}{|c|c|c|c|}
\hline 46 & $\begin{array}{l}\text { Stripping of } \\
\text { photoresist and FC } \\
\text { after DRIE } \\
\text { (\#strip121) }\end{array}$ & $\begin{array}{l}\text { NL-CLR-TePla 360M } \\
\text { Recipe } 035\end{array}$ & \\
\hline 47 & $\begin{array}{l}\text { Cleaning in } 99 \% \mathrm{HNO}_{3} \\
\text { (\#clean001) }\end{array}$ & $\begin{array}{l}\text { NL-CLR-WB14 } \\
\text { Purpose: removal of organic traces. } \\
\text { - Beaker 1: } 99 \% \mathrm{HNO}_{3} \\
\text { - Time: } 5 \mathrm{~min}\end{array}$ & \\
\hline 48 & $\begin{array}{l}\text { Cleaning in } 99 \% \mathrm{HNO}_{3} \\
\text { (\#clean001) }\end{array}$ & $\begin{array}{l}\text { NL-CLR-WB14 } \\
\text { Purpose: removal of organic traces. } \\
\text { - Beaker 2: } 99 \% \mathrm{HNO}_{3} \\
\text { - Time: } 5 \mathrm{~min}\end{array}$ & \\
\hline 49 & $\begin{array}{l}\text { Quick Dump Rinse } \\
\text { (QDR) } \\
\text { (\#rinse119) }\end{array}$ & $\begin{array}{l}\text { NL-CLR-WB14 } \\
\text { Purpose: removal of traces of chemical agents. }\end{array}$ & \\
\hline 50 & $\begin{array}{l}\text { Cleaning in } 69 \% \mathrm{HNO}_{3} \\
\text { at } 95^{\circ} \mathrm{C} \\
(\# \text { clean003) }\end{array}$ & $\begin{array}{l}\text { NL-CR-WB14 } \\
\text { Purpose: removal of metallic traces. } \\
\text { - Beaker } 3 \mathrm{a} \text { or beaker } 3 \mathrm{~b}: 69 \% \mathrm{HNO}_{3} \\
\text { - Temp.: } 95^{\circ} \mathrm{C} \\
\text { - Time: } 10 \text { min }\end{array}$ & \\
\hline 51 & $\begin{array}{l}\text { Quick Dump Rinse } \\
\text { (QDR) } \\
\text { (\#rinse119) }\end{array}$ & $\begin{array}{l}\text { NL-CLR-WB14 } \\
\text { Purpose: removal of traces of chemical agents. }\end{array}$ & \\
\hline 52 & $\begin{array}{l}\text { Substrate drying } \\
\text { (WB14) } \\
\text { (\#dry159) }\end{array}$ & $\begin{array}{l}\text { NL-CLR-WB14 } \\
\text { Batch drying of substrates }\end{array}$ & \\
\hline 53 & $\begin{array}{l}\text { Etching in HF (1\%) } \\
\text { (\#etch127) }\end{array}$ & $\begin{array}{l}\text { NL-CLR-WB15 } \\
\text { Purpose: removal of native } \mathrm{SiO}_{2} \text { from silicon wafers. } \\
\text { Beaker: } \mathrm{HF} 1 \% \\
\text { Time: } 1 \text { min }\end{array}$ & \\
\hline 54 & $\begin{array}{l}\text { Quick Dump Rinse } \\
\text { (QDR) } \\
\text { (\#rinse119) }\end{array}$ & $\begin{array}{l}\text { NL-CLR-WB15 } \\
\text { Purpose: removal of traces of chemical agents. }\end{array}$ & \\
\hline 55 & $\begin{array}{l}\text { Substrate drying } \\
\text { (WB15) } \\
\text { (\#dry160) }\end{array}$ & $\begin{array}{l}\text { NL-CLR-WB15 } \\
\text { Batch drying of substrates }\end{array}$ & \\
\hline 56 & $\begin{array}{l}\text { Dehydration bake } \\
\text { (\#lith102) }\end{array}$ & $\begin{array}{l}\text { NL-CLR-WB21/22 } \\
\text { Dehydration bake at hotplate } \\
\text { - Temp.: } 120^{\circ} \mathrm{C} \\
\text { - Time: } 5 \text { min }\end{array}$ & \\
\hline 57 & $\begin{array}{l}\text { Priming (liquid) } \\
\text { (\#lith101) }\end{array}$ & $\begin{array}{l}\text { NL-CLR-WB21/22 } \\
\text { Primer: Hexamethyldisilazane (HMDS) } \\
\text { Use spin coater: } \\
\text { - Program: } 4000 \text { (4000rpm, } 30 \mathrm{sec})\end{array}$ & \\
\hline 58 & $\begin{array}{l}\text { Coating Olin Oir 906- } \\
\mathbf{1 2} \\
(\# \text { lith104) }\end{array}$ & $\begin{array}{l}\text { NL-CLR-WB21 } \\
\text { Coating: Primus Spinner } \\
\text { - Olin OIR 906-12 } \\
\text { - Spin Program: } 4000 \text { (4000rpm, 30sec) } \\
\text { Prebake: hotplate } \\
\text { - Time: } 60 \mathrm{sec} \\
\text { - Temp.: } 95^{\circ} \mathrm{C}\end{array}$ & \\
\hline 59 & $\begin{array}{l}\text { Alignment \& exposure } \\
\text { Olin 906-12 } \\
\text { (\#lith120) }\end{array}$ & $\begin{array}{l}\text { NL-CLR- EV620 } \\
\text { Electronic Vision Group EV620 Mask Aligner } \\
\text { - Hg lamp: } 12 \mathrm{~mW} / \mathrm{cm}^{2} \\
\text { - Exposure time: } 3 \mathrm{sec}\end{array}$ & $\begin{array}{l}\text { Vacuum + } \\
\text { hard contact }\end{array}$ \\
\hline
\end{tabular}




\begin{tabular}{|c|c|c|c|c|c|}
\hline 60 & $\begin{array}{l}\text { Development Olin OiR } \\
\text { resist } \\
\text { (\#lith111) }\end{array}$ & \multicolumn{4}{|c|}{$\begin{array}{l}\text { NL-CLR-WB21 } \\
\text { After exposure bake: hotplate } \\
\text { - Time } 60 \mathrm{sec} \\
\text { - Temp.: } 120^{\circ} \mathrm{C} \\
\text { Development: developer: OPD } 4262 \\
\text { - } 30 \mathrm{sec} \text { in beaker } 1 \\
\cdot 15-30 \mathrm{sec} \text { in beaker } 2\end{array}$} \\
\hline 61 & $\begin{array}{l}\text { Quick Dump Rinse } \\
\text { (QDR) } \\
\text { (\#rinse119) }\end{array}$ & \multicolumn{4}{|c|}{$\begin{array}{l}\text { NL-CLR-WB21 } \\
\text { Purpose: removal of traces of chemical agents. }\end{array}$} \\
\hline 62 & $\begin{array}{l}\text { Substrate drying } \\
\text { (\#dry120) }\end{array}$ & \multicolumn{4}{|c|}{$\begin{array}{l}\text { NL-CLR-WB21 } \\
\text { Single substrate drying }\end{array}$} \\
\hline 63 & $\begin{array}{l}\text { Post bake Olin OiR } \\
\text { resist } \\
\text { (\#lith109) }\end{array}$ & \multicolumn{4}{|c|}{$\begin{array}{l}\text { NL-CLR-WB21 } \\
\text { Post bake: Hotplate } \\
\text { - Temp.: } 120^{\circ} \mathrm{C} \\
\text { - Time: } 10 \mathrm{~min}\end{array}$} \\
\hline 64 & $\begin{array}{l}\text { Inspection by optical } \\
\text { microscope } \\
\text { (\#metro101) }\end{array}$ & \multicolumn{4}{|c|}{$\begin{array}{l}\text { NL-CLR- Nikon Microscope } \\
\text { - Dedicated microscope for lithography inspection }\end{array}$} \\
\hline 65 & $\begin{array}{l}\text { Plasma etching of } \\
\text { silicon (B-HARS) } \\
\text { (\#etch158) }\end{array}$ & $\begin{array}{l}\text { NL-CLR-Adixer } \\
\text { B-HARS Stand } \\
\text { SH temp: } 10^{\circ} \mathrm{C} \\
\text { Parameters } \\
\text { Gas } \\
\text { Flow [sccm] } \\
\text { Time [sec] } \\
\text { Priority } \\
\text { APC \% } \\
\text { ICP [watt] } \\
\text { CCP LF [Watt] } \\
\text { Pulsed [msec] }\end{array}$ & $\begin{array}{l}\text { MS } 100 \mathrm{SE} \\
\text { os: } 200 \mathrm{~mm} \text { - } \\
\text { Etch } \\
\mathrm{SF}_{6} \\
250 \\
3 \\
2 \\
100 \\
1500 \\
80 \\
10 \text { on } / 90 \text { off }\end{array}$ & $\begin{array}{l}\text { le pres.: } 10 \mathrm{mbar} \\
\text { Deposition } \\
\mathrm{C}_{4} \mathrm{~F}_{8} \\
200 \\
1 \\
1 \\
100 \\
1500 \\
80 \\
10 \text { on/90 off }\end{array}$ & $\begin{array}{l}\text { Variable time: } \\
+/-3 \mu \mathrm{m} / \mathrm{min} \\
\text { etch rate }\end{array}$ \\
\hline 66 & $\begin{array}{l}\text { Stripping of } \\
\text { Photoresist and FC } \\
\text { after DRIE } \\
\text { (\#strip121) }\end{array}$ & \multicolumn{3}{|c|}{$\begin{array}{l}\text { NL-CLR-TePla 360M } \\
\text { Recipe } 035\end{array}$} & \\
\hline 67 & $\begin{array}{l}\text { Cleaning in } 99 \% \mathrm{HNO}_{3} \\
\text { (\#clean001) }\end{array}$ & \multicolumn{4}{|c|}{$\begin{array}{l}\text { NL-CLR-WB14 } \\
\text { Purpose: removal of organic traces. } \\
\text { - Beaker 1: } 99 \% \mathrm{HNO}_{3} \\
\text { - Time: } 5 \mathrm{~min}\end{array}$} \\
\hline 68 & $\begin{array}{l}\text { Cleaning in } 99 \% \mathrm{HNO}_{3} \\
\text { (\#clean001) }\end{array}$ & \multicolumn{3}{|c|}{$\begin{array}{l}\text { NL-CLR-WB14 } \\
\text { Purpose: removal of organic traces. } \\
\text { - Beaker 2: } 99 \% \mathrm{HNO}_{3} \\
\text { - Time: } 5 \mathrm{~min}\end{array}$} & \\
\hline 69 & $\begin{array}{l}\text { Quick Dump Rinse } \\
\text { (QDR) } \\
\text { (\#rinse119) }\end{array}$ & \multicolumn{4}{|c|}{$\begin{array}{l}\text { NL-CLR-WB14 } \\
\text { Purpose: removal of traces of chemical agents. }\end{array}$} \\
\hline 70 & $\begin{array}{l}\text { Cleaning in } 69 \% \mathrm{HNO}_{3} \\
\text { at } 95{ }^{\circ} \mathrm{C} \\
(\# \text { clean003) }\end{array}$ & \multicolumn{4}{|c|}{$\begin{array}{l}\text { NL-CR-WB14 } \\
\text { Purpose: removal of metallic traces. } \\
\text { - Beaker } 3 \mathrm{a} \text { or beaker } 3 \mathrm{~b}: 69 \% \mathrm{HNO}_{3} \\
\text { - Temp.: } 95^{\circ} \mathrm{C} \\
\text { - Time: } 10 \text { min }\end{array}$} \\
\hline 71 & $\begin{array}{l}\text { Quick Dump Rinse } \\
\text { (QDR) } \\
\text { (\#rinse119) }\end{array}$ & \multicolumn{4}{|c|}{$\begin{array}{l}\text { NL-CLR-WB14 } \\
\text { Purpose: removal of traces of chemical agents. }\end{array}$} \\
\hline
\end{tabular}




\begin{tabular}{|c|c|c|c|}
\hline 72 & $\begin{array}{l}\text { Etching in HF (1\%) } \\
\text { (\#etch127) }\end{array}$ & $\begin{array}{l}\text { NL-CLR-WB15 } \\
\text { Purpose: removal of native } \mathrm{SiO}_{2} \text { from silicon wafers. } \\
\text { Beaker: } \mathrm{HF} 1 \% \\
\text { Time: } 1 \text { min }\end{array}$ & \\
\hline 73 & $\begin{array}{l}\text { Quick Dump Rinse } \\
\text { (QDR) } \\
\text { (\#rinse119) }\end{array}$ & $\begin{array}{l}\text { NL-CLR-WB15 } \\
\text { Purpose: removal of traces of chemical agents. }\end{array}$ & \\
\hline 74 & $\begin{array}{l}\text { Substrate drying } \\
\text { (WB15) (\#dry160) }\end{array}$ & $\begin{array}{l}\text { NL-CLR-WB15 } \\
\text { Batch drying of substrates }\end{array}$ & \\
\hline 75 & $\begin{array}{l}\text { Solid Source Diffusion } \\
\text { (SSD) of Boron } \\
\text { at } 1050^{\circ} \mathrm{C}(\# \text { dopi } 104)\end{array}$ & $\begin{array}{l}\text { NL-CLR-SSD furnace Tempress B1 } \\
\text { Standby temperature: } 700^{\circ} \mathrm{C} \\
\text { Program: SSD-1050 } \\
\mathrm{B}_{2} \mathrm{O}_{5} \text { deposition } \\
\text { - Temp } 900^{\circ} \mathrm{C} \\
\text { - Gas } \mathrm{O}_{2}, 2 \mathrm{SLM} \\
\text { - Gas } \mathrm{N}_{2}, 2 \mathrm{SLM} \\
\text { - Time } 15 \mathrm{~min} \\
\\
\text { Anneal (soak) } \\
\text { - Temp.: } 1050^{\circ} \mathrm{C} \\
\text { - Ramp up: } 5^{\circ} \mathrm{C} / \mathrm{min} \\
\text { - Cool down: } 7.5^{\circ} \mathrm{C} / \mathrm{min} \\
\text { - Gas } \mathrm{N}_{2}: 4 \mathrm{SLM} \\
\text { - Time: variable }\end{array}$ & $\frac{\text { N-type ONLY }}{15 \min }$ \\
\hline 76 & $\begin{array}{l}\text { Etching in BHF (1:7) } \\
\text { (\#etch119) }\end{array}$ & NL-CLR-WB06 & $\frac{\text { N-type ONLY }}{10 \min }$ \\
\hline 77 & $\begin{array}{l}\text { Quick Dump Rinse } \\
\text { (QDR) } \\
\text { (\#rinse119) }\end{array}$ & $\begin{array}{l}\text { NL-CLR-WB06 } \\
\text { Purpose: removal of traces of chemical agents. }\end{array}$ & N-type ONLY \\
\hline 78 & $\begin{array}{l}\text { Substrate drying } \\
\text { (WB06-WB08) } \\
\text { (\#dry157) }\end{array}$ & $\begin{array}{l}\text { NL-CLR-WB06-WB08 } \\
\text { Batch drying of substrates }\end{array}$ & N-type ONLY \\
\hline 79 & $\begin{array}{l}\text { Dry Oxidation of } \\
\text { silicon at } 800^{\circ} \mathrm{C} \\
\text { (\#film126) }\end{array}$ & $\begin{array}{l}\text { NL-CLR-Furnace B3 } \\
\text { - Standby temperature: } 800{ }^{\circ} \mathrm{C} \\
\text { - Program: Ox } 800 \\
\text { - Temp.: } 800{ }^{\circ} \mathrm{C} \\
\text { - Gas: } \mathrm{O}_{2} \\
\text { - Flow: } 1 \mathrm{l} / \mathrm{min}\end{array}$ & $\frac{\text { N-type ONLY }}{15 \min }$ \\
\hline 80 & $\begin{array}{l}\text { Etching in BHF (1:7) } \\
\text { (\#etch119) }\end{array}$ & NL-CLR-WB06 & $\begin{array}{l}\text { N-type ONLY } \\
15 \text { min // Until } \\
\text { hydrophobic }\end{array}$ \\
\hline 81 & $\begin{array}{l}\text { Quick Dump Rinse } \\
\text { (QDR) } \\
\text { (\#rinse119) }\end{array}$ & $\begin{array}{l}\text { NL-CLR-WB06 } \\
\text { Purpose: removal of traces of chemical agents. }\end{array}$ & N-type ONLY \\
\hline 82 & $\begin{array}{l}\text { Substrate drying } \\
\text { (WB06-WB08) } \\
\text { (\#dry157) }\end{array}$ & $\begin{array}{l}\text { NL-CLR-WB06-WB08 } \\
\text { Batch drying of substrates }\end{array}$ & N-type ONLY \\
\hline 83 & $\begin{array}{l}\text { LPCVD of PSG (TEOS) } \\
\text { (\#film188) }\end{array}$ & $\begin{array}{l}\text { NL-CLR-LPCVD H4 } \\
\text { Program: N8-PSG } \\
\text { - } 50 \mathrm{sccm} \text { TEOS } \\
\cdot 330 \mathrm{sccm} \mathrm{PH}_{3}\end{array}$ & P-type ONLY \\
\hline 84 & 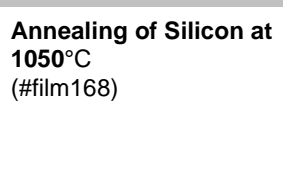 & $\begin{array}{l}\text { NL-CLR-Furnace B3 } \\
\text { - Standby temperature: } 800^{\circ} \mathrm{C} \\
\text { - Program: ANN1050 } \\
\text { - Temp.: } 1050^{\circ} \mathrm{C} \\
\text { - Gas: } \mathrm{N}_{2} \\
\text { - Flow: } 21 / \text { min }\end{array}$ & $\begin{array}{l}\text { P-type ONLY } \\
15 \text { min }\end{array}$ \\
\hline
\end{tabular}




\begin{tabular}{|c|c|c|c|}
\hline 85 & $\begin{array}{l}\text { Etching in BHF (1:7) } \\
\text { (\#etch124) }\end{array}$ & NL-CLR-WB06 & $\begin{array}{l}\text { P-type ONLY } \\
15 \text { min // Until } \\
\text { hydrophobic }\end{array}$ \\
\hline 86 & $\begin{array}{l}\text { Quick Dump Rinse } \\
\text { (QDR) } \\
\text { (\#rinse119) }\end{array}$ & $\begin{array}{l}\text { NL-CLR-WB06 } \\
\text { Purpose: removal of traces of chemical agents. }\end{array}$ & P-type ONLY \\
\hline 87 & $\begin{array}{l}\text { Substrate drying } \\
\text { (\#dry120) }\end{array}$ & $\begin{array}{l}\text { NL-CLR-WB06-WB08 } \\
\text { Batch drying of substrates }\end{array}$ & P-type ONLY \\
\hline 88 & $\begin{array}{l}\text { DRIE of } \mathrm{SiO}_{2}, \text { SiRN } \\
\text { (\#etch157) }\end{array}$ & $\begin{array}{l}\text { NL-CLR- AdixenDE } \\
\text { Layer thickness: }<5 \mu \mathrm{m} \mathrm{SiO}_{2} \\
\text { SH Temp: }-10^{\circ} \mathrm{C}-\mathrm{Pos}: 120 \mathrm{~mm}-\mathrm{He} \text { pres: } 10 \mathrm{mbar} \\
\text { Flows } \mathrm{C}_{4} \mathrm{~F}_{8}: 20 \mathrm{sccm}-\mathrm{CH}_{4}: 15 \mathrm{sccm}-\mathrm{He}: 150 \mathrm{sccm} \\
\text { Pressure: } 8.510^{-3} \mathrm{mbar} \\
\text { ICP: } 2800 \text { Watt } \\
\text { CCP: } 350 \text { Watt (RF) }\end{array}$ & $\begin{array}{l}\text { Backside only, } \\
45 \mathrm{sec}\end{array}$ \\
\hline 89 & $\begin{array}{l}\text { Chamber clean Adixen } \\
\text { DE } \\
\text { (\#etch201) }\end{array}$ & $\begin{array}{l}\text { NL-CLR-AdixenDE } \\
\text { Chamber clean to remove fluorocarbon } \\
\text { Needed after every } 20 \text { min processing } \\
\text { SH Temp: any- Pos: } 150 \mathrm{~mm} \text { - He pres.: } 10 \text { mbar } \\
\text { Flow } \mathrm{O}_{2}: 200 \text { sccm } \\
\text { APC } 100 \% \text { / Pressure: } 1.510^{-2-} 6.0^{*} 10^{-6} \mathrm{mbar} \\
\text { ICP: } 3000 \text { Watt } \\
\text { CCP: } 50 \text { Watt (RF) } \\
\text { Clean with a plain silicon wafer in the etch tool } \\
\text { Cleaning time: } 30 \text { minutes }\end{array}$ & \\
\hline 90 & $\begin{array}{l}\text { Stripping of } \\
\text { Photoresist and FC } \\
\text { after DRIE } \\
\text { (\#strip121) }\end{array}$ & $\begin{array}{l}\text { NL-CLR-TePla } 360 \mathrm{M} \\
\text { Recipe } 035\end{array}$ & \\
\hline 91 & $\begin{array}{l}\text { Etching 1\% HF } \\
\text { (\#etch210) }\end{array}$ & $\begin{array}{l}\text { NL-CLR-WB06 } \\
\text { use Beaker HF with } 1 \%\end{array}$ & $\begin{array}{l}1 \mathrm{~min} / / \text { Wafer } \\
\text { MUST be } \\
\text { hydrophobic! }\end{array}$ \\
\hline 92 & $\begin{array}{l}\text { Quick Dump Rinse } \\
\text { (QDR) } \\
\text { (\#rinse119) }\end{array}$ & $\begin{array}{l}\text { NL-CLR-WB06 } \\
\text { Purpose: removal of traces of chemical agents. }\end{array}$ & \\
\hline 93 & $\begin{array}{l}\text { Substrate drying } \\
\text { (\#dry120) }\end{array}$ & $\begin{array}{l}\text { NL-CLR-WB06-WB08 } \\
\text { Batch drying of substrates }\end{array}$ & \\
\hline 94 & $\begin{array}{l}\text { Sputtering of Al } \\
\text { (\#film122) }\end{array}$ & $\begin{array}{l}\text { NL-CLR-Oxford PL400 } \\
\text { - Program: } 1000 \mathrm{~nm} \text { Al position } 1 \\
\text { - Pressure: } 10 \mathrm{mTorr} \\
\text { Deposition rate }(100 \mathrm{~mm} \text { wafer })=820 \mathrm{~nm} / \mathrm{min}\end{array}$ & $\begin{array}{l}\text { Backside: } \\
1: 13 \text { min } \\
\text { Front side: } \\
2 \times 36 \text { sec with } \\
\text { shadow mask }\end{array}$ \\
\hline
\end{tabular}




\section{A.2. Process flow axial junctions}

Process flow for cryogenic etching of axial $\mathrm{n}^{+} / \mathrm{p}$ junctions using spin-on-dopant of phosphorus.

\section{A.2.1 Wafer selection}

\begin{tabular}{|ll} 
Substrate Silicon $<100>$ OSP & Orientation: $<100>$ \\
P-type & Diameter: $100 \mathrm{~mm}$ \\
(\#subs108) & Thickness: $525 \mu \mathrm{m} \pm 25$ \\
& Polished: Single side polished \\
& Resistivity: $5-10 \Omega \mathrm{cm}$ \\
& Type: $P$
\end{tabular}

\section{A.2.2. Process flow}

\begin{tabular}{|c|c|c|c|}
\hline \multicolumn{2}{|r|}{ Process } & & \multirow[t]{2}{*}{ Comment } \\
\hline 1 & $\begin{array}{l}\text { Cleaning in } 99 \% \mathrm{HNO}_{3} \\
\text { (\#clean001) }\end{array}$ & $\begin{array}{l}\text { NL-CLR-WB14 } \\
\text { Purpose: removal of organic traces. } \\
\text { - Beaker } 1: 99 \% \mathrm{HNO}_{3} \\
\text { - Time: } 5 \text { min }\end{array}$ & \\
\hline 2 & $\begin{array}{l}\text { Cleaning in } 99 \% \mathrm{HNO}_{3} \\
\text { (\#clean001) }\end{array}$ & $\begin{array}{l}\text { NL-CLR-WB14 } \\
\text { Purpose: removal of organic traces. } \\
\text { - Beaker 2: } 99 \% \mathrm{HNO}_{3} \\
\text { - Time: } 5 \text { min }\end{array}$ & \\
\hline 3 & $\begin{array}{l}\text { Quick Dump Rinse } \\
\text { (QDR) } \\
\text { (\#rinse119) }\end{array}$ & $\begin{array}{l}\text { NL-CLR- WB14 } \\
\text { Purpose: removal of traces of chemical agents. }\end{array}$ & \\
\hline 4 & $\begin{array}{l}\text { Cleaning in } 69 \% \mathrm{HNO}_{3} \\
\text { at } 95^{\circ} \mathrm{C} \\
(\# \text { clean003) }\end{array}$ & $\begin{array}{l}\text { NL-CR-WB14 } \\
\text { Purpose: removal of metallic traces. } \\
\text { - Beaker } 3 \mathrm{a} \text { or beaker } 3 \mathrm{~b}: 69 \% \mathrm{HNO}_{3} \\
\text { - Temp.: } 95^{\circ} \mathrm{C} \\
\text { - Time: } 10 \mathrm{~min}\end{array}$ & \\
\hline 5 & $\begin{array}{l}\text { Quick Dump Rinse } \\
\text { (QDR) } \\
\text { (\#rinse119) }\end{array}$ & $\begin{array}{l}\text { NL-CLR- WB14 } \\
\text { Purpose: removal of traces of chemical agents. }\end{array}$ & \\
\hline 6 & $\begin{array}{l}\text { Substrate drying } \\
\text { (WB14) } \\
\text { (\#dry159) }\end{array}$ & $\begin{array}{l}\text { NL-CLR-WB14 } \\
\text { Batch drying of substrates }\end{array}$ & \\
\hline 7 & $\begin{array}{l}\text { Etching in HF (1\%) } \\
\text { (\#etch127) }\end{array}$ & $\begin{array}{l}\text { NL-CLR-WB15 } \\
\text { Purpose: removal of native } \mathrm{SiO}_{2} \text { from silicon wafers. } \\
\text { Beaker: } \mathrm{HF} 1 \% \\
\text { Time: } 1 \text { min }\end{array}$ & \\
\hline 8 & $\begin{array}{l}\text { Quick Dump Rinse } \\
\text { (QDR) } \\
\text { (\#rinse119) }\end{array}$ & $\begin{array}{l}\text { NL-CLR- WB15 } \\
\text { Purpose: removal of traces of chemical agents. }\end{array}$ & \\
\hline 9 & $\begin{array}{l}\text { Substrate drying } \\
\text { (WB15) } \\
\text { (\#dry160) }\end{array}$ & $\begin{array}{l}\text { NL-CLR-WB15 } \\
\text { Batch drying of substrates }\end{array}$ & \\
\hline 10 & $\begin{array}{l}\text { LPCVD of SiRN }(250 \\
\text { MPa) } \\
(\# \text { film155) }\end{array}$ & $\begin{array}{l}\text { NL-CLR-Tempress LPCVD G4 } \\
\text { Program: N2 LSNIT-G4 (200 mTorr) } \\
\text { - } \mathrm{SiH}_{2} \mathrm{Cl}_{2} \text { flow: } 150 \mathrm{sccm} \\
\text { - } \mathrm{NH}_{3} \text { flow: } 50 \mathrm{sccm}\end{array}$ & $\begin{array}{l}100 \mathrm{~nm} \text { in } 12 \\
\min \end{array}$ \\
\hline
\end{tabular}




\begin{tabular}{|c|c|c|c|}
\hline 11 & $\begin{array}{l}\text { Inspection of } \\
\text { LPCVD/PECVD layers } \\
\text { (\#metro113) }\end{array}$ & $\begin{array}{l}\text { NL-CLR-cold light source } \\
\text { Particle and haze inspection by using cold light source }\end{array}$ & \\
\hline 12 & $\begin{array}{l}\text { Ellipsometer } \\
\text { measurement } \\
\text { (\#metro107) }\end{array}$ & NL-CLR-Woollam M-2000 ellipsometer & \\
\hline 13 & $\begin{array}{l}\text { DRIE of } \mathrm{SiO}_{2}, \text { SiRN } \\
\text { (\#etch157) }\end{array}$ & $\begin{array}{l}\text { NL-CLR- AdixenDE } \\
\text { Layer thickness: }<5 \mu \mathrm{m} \mathrm{SiO}_{2} \\
\text { SH Temp: }-10^{\circ} \mathrm{C}-\mathrm{Pos}: 120 \mathrm{~mm}-\mathrm{He} \text { pres.: } 10 \mathrm{mbar} \\
\text { Flows } \mathrm{C}_{4} \mathrm{~F}_{8}: 20 \mathrm{sccm}-\mathrm{CH}_{4}: 15 \mathrm{sccm}-\mathrm{He}: 150 \mathrm{sccm} \\
\text { Pressure: } 8.510^{-3} \mathrm{mbar} \\
\text { ICP: } 2800 \text { Watt } \\
\text { CCP: } 350 \text { Watt (RF) }\end{array}$ & $\begin{array}{l}\text { Front side } \\
\text { only, } 45 \mathrm{sec}\end{array}$ \\
\hline 14 & $\begin{array}{l}\text { Chamber clean } \\
\text { AdixenDE } \\
\text { (\#etch201) }\end{array}$ & $\begin{array}{l}\text { NL-CLR-AdixenDE } \\
\text { Chamber clean to remove fluorocarbon } \\
\text { Needed after every } 20 \text { min processing } \\
\text { SH Temp: any- Pos: } 150 \mathrm{~mm}-\text { He pres.: } 10 \mathrm{mbar} \\
\text { Flow } \mathrm{O}_{2}: 200 \text { sccm } \\
\text { APC } 100 \% \text { / Pressure: } 1.510^{-2-} 6.0^{*} 10^{-6} \mathrm{mbar} \\
\text { ICP: } 3000 \text { Watt } \\
\text { CCP: } 50 \text { Watt (RF) } \\
\text { Clean with a plain silicon wafer in the etch tool } \\
\text { Cleaning time: } 30 \text { minutes }\end{array}$ & \\
\hline 15 & $\begin{array}{l}\text { Stripping of } \\
\text { photoresist and FC } \\
\text { after DRIE } \\
\text { (\#strip121) }\end{array}$ & $\begin{array}{l}\text { NL-CLR-TePla 360M } \\
\text { Recipe } 035\end{array}$ & \\
\hline 16 & $\begin{array}{l}\text { Cleaning in } 99 \% \mathrm{HNO}_{3} \\
\text { (\#clean001) }\end{array}$ & $\begin{array}{l}\text { NL-CLR-WB14 } \\
\text { Purpose: removal of organic traces. } \\
\text { - Beaker 1: } 99 \% \mathrm{HNO}_{3} \\
\text { - Time: } 5 \text { min }\end{array}$ & \\
\hline 17 & $\begin{array}{l}\text { Cleaning in } 99 \% \mathrm{HNO}_{3} \\
\text { (\#clean001) }\end{array}$ & $\begin{array}{l}\text { NL-CLR-WB14 } \\
\text { Purpose: removal of organic traces. } \\
\text { - Beaker 2: } 99 \% \mathrm{HNO}_{3} \\
\text { - Time: } 5 \text { min }\end{array}$ & \\
\hline 18 & $\begin{array}{l}\text { Quick Dump Rinse } \\
\text { (QDR) } \\
\text { (\#rinse119) }\end{array}$ & $\begin{array}{l}\text { NL-CLR-WB14 } \\
\text { Purpose: removal of traces of chemical agents. }\end{array}$ & \\
\hline 19 & $\begin{array}{l}\text { Cleaning in } 69 \% \mathrm{HNO}_{3} \\
\text { at } 95{ }^{\circ} \mathrm{C} \\
(\# \text { clean003) }\end{array}$ & $\begin{array}{l}\text { NL-CR-WB14 } \\
\text { Purpose: removal of metallic traces. } \\
\text { - Beaker } 3 \mathrm{a} \text { or beaker } 3 \mathrm{~b}: 69 \% \mathrm{HNO}_{3} \\
\text { - Temp.: } 95{ }^{\circ} \mathrm{C} \\
\text { - Time: } 10 \text { min }\end{array}$ & \\
\hline 20 & $\begin{array}{l}\text { Quick Dump Rinse } \\
\text { (QDR) } \\
\text { (\#rinse119) }\end{array}$ & $\begin{array}{l}\text { NL-CLR-WB14 } \\
\text { Purpose: removal of traces of chemical agents. }\end{array}$ & \\
\hline 21 & $\begin{array}{l}\text { Substrate drying } \\
\text { (WB14) } \\
\text { (\#dry159) }\end{array}$ & $\begin{array}{l}\text { NL-CLR-WB14 } \\
\text { Batch drying of substrates }\end{array}$ & \\
\hline 22 & $\begin{array}{l}\text { Etching in HF (1\%) } \\
\text { (\#etch127) }\end{array}$ & $\begin{array}{l}\mathrm{NL}-\mathrm{CLR}-\mathrm{WB} 15 \\
\text { Purpose: removal of native } \mathrm{SiO}_{2} \text { from silicon wafers. } \\
\text { Beaker: } \mathrm{HF} 1 \% \\
\text { Time: } 1 \text { min }\end{array}$ & \\
\hline 23 & $\begin{array}{l}\text { Quick Dump Rinse } \\
\text { (QDR) } \\
\text { (\#rinse119) }\end{array}$ & $\begin{array}{l}\text { NL-CLR-WB15 } \\
\text { Purpose: removal of traces of chemical agents. }\end{array}$ & \\
\hline 24 & Substrate drying & NL-CLR-WB15 & \\
\hline
\end{tabular}




\begin{tabular}{|c|c|c|c|}
\hline & $\begin{array}{l}\text { (WB15) } \\
\text { (\#dry160) }\end{array}$ & Batch drying of substrates & \\
\hline 25 & $\begin{array}{l}\text { Spin-on-dopant } \\
\text { (\#film) }\end{array}$ & $\begin{array}{l}\text { NL-CLR-WB22 } \\
\text { - Filmtronics P509 } \\
\text { - Program: } 6000 \text { rpm, } 30 \mathrm{sec}\end{array}$ & $\begin{array}{l}\text { Check expiry } \\
\text { date! }\end{array}$ \\
\hline 26 & $\begin{array}{l}\text { Anneal at } 1100{ }^{\circ} \mathrm{C} \\
(\# \text { film169) }\end{array}$ & $\begin{array}{l}\text { NL-CLR-Furnace } \mathrm{B} 3 \\
\text { - Standby temperature: } 800^{\circ} \mathrm{C} \\
\text { - Temp.: } 1100^{\circ} \mathrm{C} \\
\text { - Gas: } \mathrm{N}_{2} \\
\text { - Flow: } 2 \mathrm{l} / \mathrm{min}\end{array}$ & $\begin{array}{l}\text { Experimental } \\
\text { Check with } \\
\text { operator! } \\
\text { Time: } 60 \mathrm{~min} \\
\text { (+/- } 3.6 \mu \mathrm{m} \\
\text { junction) }\end{array}$ \\
\hline 27 & $\begin{array}{l}\text { Etching in BHF (1:7) } \\
\text { (\#etch124) }\end{array}$ & $\begin{array}{l}\text { NL-CLR-WB06 } \\
\text { Use dedicated beaker BHF }(1: 7) \\
\text { Temp.: Room temperature }\end{array}$ & $\begin{array}{l}15 \text { min // Until } \\
\text { hydrophobic }\end{array}$ \\
\hline 28 & $\begin{array}{l}\text { Quick Dump Rinse } \\
\text { (QDR) } \\
\text { (\#rinse119) }\end{array}$ & $\begin{array}{l}\text { NL-CLR-WB06 } \\
\text { Purpose: removal of traces of chemical agents. }\end{array}$ & \\
\hline 29 & $\begin{array}{l}\text { Substrate drying } \\
\text { (\#dry120) }\end{array}$ & $\begin{array}{l}\text { NL-CLR-WB06-08 } \\
\text { Batch drying of substrates }\end{array}$ & \\
\hline 30 & $\begin{array}{l}\text { DRIE of } \mathrm{SiO}_{2}, \mathrm{SiRN} \\
\text { (\#etch157) }\end{array}$ & $\begin{array}{l}\text { NL-CLR- AdixenDE } \\
\text { Layer thickness: < } \mu \mathrm{m} \text { SiO2 } \\
\text { SH Temp: }-10^{\circ} \mathrm{C}-\mathrm{Pos}: 120 \mathrm{~mm}-\mathrm{He} \text { pres.: } 10 \mathrm{mbar} \\
\text { Flows } \mathrm{C}_{4} \mathrm{~F}_{8}: 20 \mathrm{sccm}-\mathrm{CH}_{4}: 15 \mathrm{sccm}-\mathrm{He}: 150 \mathrm{sccm} \\
\text { Pressure: } 8.510^{-3} \mathrm{mbar} \\
\text { ICP: } 2800 \text { Watt } \\
\text { CCP: } 350 \text { Watt (RF) }\end{array}$ & $\begin{array}{l}\text { Backside only, } \\
45 \mathrm{sec}\end{array}$ \\
\hline 31 & $\begin{array}{l}\text { Chamber clean Adixen } \\
\text { DE } \\
\text { (\#etch201) }\end{array}$ & $\begin{array}{l}\text { NL-CLR-AdixenDE } \\
\text { Chamber clean to remove fluorocarbon } \\
\text { Needed after every } 20 \text { min processing } \\
\text { SH Temp: any- Pos: } 150 \mathrm{~mm} \text { - He pres.: } 10 \text { mbar } \\
\text { Flow } \mathrm{O}_{2}: 200 \text { sccm } \\
\text { APC } 100 \% \text { / Pressure: } 1.510^{-2}-6.0^{*} 10^{-6} \mathrm{mbar} \\
\text { ICP: } 3000 \text { Watt } \\
\text { CCP: } 50 \text { Watt (RF) }\end{array}$ & \\
\hline 32 & $\begin{array}{l}\text { Stripping of } \\
\text { Photoresist and FC } \\
\text { after DRIE } \\
\text { (\#strip121) }\end{array}$ & $\begin{array}{l}\text { NL-CLR-TePla } 360 \mathrm{M} \\
\text { Recipe } 035\end{array}$ & \\
\hline 33 & $\begin{array}{l}\text { Dehydration bake } \\
\text { (\#lith102) }\end{array}$ & $\begin{array}{l}\text { NL-CLR-WB21/22 } \\
\text { Dehydration bake at hotplate } \\
\text { - Temp.: } 120^{\circ} \mathrm{C} \\
\text { - Time: } 5 \mathrm{~min}\end{array}$ & \\
\hline 34 & $\begin{array}{l}\text { Priming (liquid) } \\
\text { (\#lith101) }\end{array}$ & $\begin{array}{l}\text { NL-CLR-WB21/22 } \\
\text { Primer: Hexamethyldisilazane (HMDS) } \\
\text { Use spin coater: } \\
\text { - Program: } 4000 \text { (4000rpm, 30sec) }\end{array}$ & \\
\hline 35 & $\begin{array}{l}\text { Coating Olin Oir 906- } \\
12 \\
(\# \text { lith104) }\end{array}$ & $\begin{array}{l}\text { NL-CLR-WB21 } \\
\text { Coating: Primus Spinner } \\
\text { - Olin OIR } 906-12 \\
\text { - Spin Program: } 4000 \text { (4000rpm, 30sec) } \\
\text { Prebake: hotplate } \\
\text { - Time: } 60 \mathrm{sec} \\
\text { - Temp.: } 95^{\circ} \mathrm{C}\end{array}$ & \\
\hline 36 & $\begin{array}{l}\text { Alignment \& exposure } \\
\text { Olin 906-12 } \\
\text { (\#lith120) }\end{array}$ & $\begin{array}{l}\text { NL-CLR- EV620 } \\
\text { Electronic Vision Group EV620 Mask Aligner } \\
\text { - Hg lamp: } 12 \mathrm{~mW} / \mathrm{cm}^{2} \\
\text { - Exposure time: } 3 \mathrm{sec}\end{array}$ & $\begin{array}{l}\text { Vacuum + } \\
\text { hard contact }\end{array}$ \\
\hline
\end{tabular}




\begin{tabular}{|c|c|c|c|}
\hline 37 & $\begin{array}{l}\text { Development Olin OiR } \\
\text { resist } \\
\text { (\#lith111) }\end{array}$ & $\begin{array}{l}\text { NL-CLR-WB21 } \\
\text { After exposure bake: hotplate } \\
\text { - Time } 60 \text { sec } \\
\text { - Temp.: } 120^{\circ} \mathrm{C} \\
\text { Development: developer: OPD } 4262 \\
\text { - } 30 \mathrm{sec} \text { in beaker } 1 \\
\text { - } 15-30 \mathrm{sec} \text { in beaker } 2\end{array}$ & \\
\hline 38 & $\begin{array}{l}\text { Quick Dump Rinse } \\
\text { (QDR) } \\
\text { (\#rinse119) }\end{array}$ & $\begin{array}{l}\text { NL-CLR-WB21 } \\
\text { Purpose: removal of traces of chemical agents. }\end{array}$ & \\
\hline 39 & $\begin{array}{l}\text { Substrate drying } \\
\text { (\#dry120) }\end{array}$ & $\begin{array}{l}\text { NL-CLR-WB21 } \\
\text { Single substrate drying }\end{array}$ & \\
\hline 40 & $\begin{array}{l}\text { Post bake Olin OiR } \\
\text { resist } \\
\text { (\#lith109) }\end{array}$ & $\begin{array}{l}\text { NL-CLR-WB21 } \\
\text { Post bake: Hotplate } \\
\text { - Temp.: } 120^{\circ} \mathrm{C} \\
\text { - Time: } 10 \mathrm{~min}\end{array}$ & \\
\hline 41 & $\begin{array}{l}\text { Inspection by optical } \\
\text { microscope } \\
\text { (\#metro101) }\end{array}$ & $\begin{array}{l}\text { NL-CLR- Nikon Microscope } \\
\text { - Dedicated microscope for lithography inspection }\end{array}$ & \\
\hline 42 & $\begin{array}{l}\text { Chamber clean } \mathrm{O}_{2} \\
\text { plasma } \\
\text { (\#)tch200) }\end{array}$ & $\begin{array}{l}\text { NL-CLR-AdixenSE } \\
\text { Chamber clean: removal of fluorocarbon residue } \\
\text { Apply always before (cryogenic) } \mathrm{SF}_{6} / \mathrm{O}_{2} \text { processing } \\
\text { SH temp: any - Pos: } 200 \mathrm{~mm}-\mathrm{He} \text { pres.: } 10 \mathrm{mbar} \\
\text { Gas: } 200 \mathrm{sccm} \mathrm{O}_{2} \\
\text { ICP: } 2000 \mathrm{~W}, \mathrm{CCP}: 50 \mathrm{~W}\end{array}$ & $30 \mathrm{~min}$ \\
\hline 43 & $\begin{array}{l}\text { DRIE of Silicon C- } \\
\text { SF }_{6} / \mathrm{O}_{2} \text { Cryogenic } \\
\text { (\#)tch177) }\end{array}$ & $\begin{array}{l}\text { NL-CLR-Adixen } \mathrm{SE} \\
\text { Temperature: }-100^{\circ} \mathrm{C} \\
\text { Gas: } 100 \mathrm{sccm} \mathrm{SF}_{6} \\
\text { Gas: } 32.5 \mathrm{sccm} \mathrm{O} \mathrm{O}_{2} \\
\text { ICP: } 1000 \mathrm{~W} \\
\text { SH: } 200 \mathrm{~mm} \\
\text { He pres.: } 10 \mathrm{mbar}\end{array}$ & $\begin{array}{l}\text { Approximately } \\
2.5-3 \mu \mathrm{m} / \mathrm{min}\end{array}$ \\
\hline 44 & $\begin{array}{l}\text { Stripping of } \\
\text { Photoresist and FC } \\
\text { after DRIE } \\
\text { (\#strip121) }\end{array}$ & $\begin{array}{l}\text { NL-CLR-TePla 360M } \\
\text { Recipe } 035\end{array}$ & \\
\hline 45 & $\begin{array}{l}\text { Cleaning in } 99 \% \mathrm{HNO}_{3} \\
\text { (\#clean001) }\end{array}$ & $\begin{array}{l}\text { NL-CLR-WB14 } \\
\text { Purpose: removal of organic traces. } \\
\text { - Beaker } 1: 99 \% \mathrm{HNO}_{3} \\
\text { - Time: } 5 \mathrm{~min}\end{array}$ & \\
\hline 46 & $\begin{array}{l}\text { Cleaning in } 99 \% \mathrm{HNO}_{3} \\
\text { (\#clean001) }\end{array}$ & $\begin{array}{l}\text { NL-CLR-WB14 } \\
\text { Purpose: removal of organic traces. } \\
\text { - Beaker } 2: 99 \% \mathrm{HNO}_{3} \\
\text { - Time: } 5 \mathrm{~min}\end{array}$ & \\
\hline 47 & $\begin{array}{l}\text { Quick Dump Rinse } \\
\text { (QDR) } \\
\text { (\#rinse119) }\end{array}$ & $\begin{array}{l}\text { NL-CLR-WB14 } \\
\text { Purpose: removal of traces of chemical agents. }\end{array}$ & \\
\hline 48 & $\begin{array}{l}\text { Cleaning in } 69 \% \mathrm{HNO}_{3} \\
\text { at } 95{ }^{\circ} \mathrm{C} \\
(\# \text { clean003) }\end{array}$ & $\begin{array}{l}\text { NL-CR-WB14 } \\
\text { Purpose: removal of metallic traces. } \\
\text { - Beaker } 3 \mathrm{a} \text { or beaker } 3 \mathrm{~b}: 69 \% \mathrm{HNO}_{3} \\
\text { - Temp.: } 95{ }^{\circ} \mathrm{C} \\
\text { - Time: } 10 \text { min }\end{array}$ & \\
\hline 49 & $\begin{array}{l}\text { Quick Dump Rinse } \\
\text { (QDR) } \\
\text { (\#rinse119) }\end{array}$ & $\begin{array}{l}\text { NL-CLR-WB14 } \\
\text { Purpose: removal of traces of chemical agents. }\end{array}$ & \\
\hline
\end{tabular}




\begin{tabular}{|c|c|c|c|}
\hline 50 & $\begin{array}{l}\text { Etching in HF (1\%) } \\
\text { (\#etch127) }\end{array}$ & $\begin{array}{l}\text { NL-CLR-WB15 } \\
\text { Purpose: removal of native } \mathrm{SiO}_{2} \text { from silicon wafers. } \\
\text { Beaker: } \mathrm{HF} 1 \% \\
\text { Time: } 1 \mathrm{~min}\end{array}$ & $\begin{array}{l}1 \mathrm{~min} / / \text { Wafer } \\
\text { MUST be } \\
\text { hydrophobic! }\end{array}$ \\
\hline 51 & $\begin{array}{l}\text { Quick Dump Rinse } \\
\text { (QDR) } \\
\text { (\#rinse119) }\end{array}$ & $\begin{array}{l}\text { NL-CLR-WB15 } \\
\text { Purpose: removal of traces of chemical agents. }\end{array}$ & \\
\hline 52 & $\begin{array}{l}\text { Substrate drying } \\
\text { (WB15) } \\
\text { (\#dry160) }\end{array}$ & $\begin{array}{l}\text { NL-CLR-WB15 } \\
\text { Batch drying of substrates }\end{array}$ & \\
\hline 53 & $\begin{array}{l}\text { Sputtering of Al } \\
(\# \text { film122) }\end{array}$ & $\begin{array}{l}\text { NL-CLR-Oxford PL400 } \\
\text { - Program: } 1000 \mathrm{~nm} \text { Al position } 1 \\
\text { - Pressure: } 10 \mathrm{mTorr} \\
\text { Deposition rate }(100 \mathrm{~mm} \text { wafer })=820 \mathrm{~nm} / \mathrm{min}\end{array}$ & $\begin{array}{l}\text { Backside: } \\
\text { 1:13 min } \\
\text { Front side: } \\
2 x 36 \text { sec with } \\
\text { shadow mask }\end{array}$ \\
\hline
\end{tabular}




\section{Dankwoord}

Na vier jaar is het dan zo ver, ik mag mijn dankwoord schrijven. Met de nadruk op 'mag', doel ik op het feit dat het een eer is om dit te kunnen doen. Na een langdurig project, waarbij je in het begin vaak nog geen idee hebt wat eruit zal komen, is het opleveren van je 'boekje' een gevoel waar je trots op kan zijn. Uiteindelijk is het een resultaat dat tot stand gekomen is door vele experimenten, discussies, presentaties en een goede dosis toeval. Tijdens mijn promotie heb ik het geluk gehad dat ik met veel personen heb mogen samenwerken en daarvoor wil ik iedereen graag bedanken in dit dankwoord.

Allereerst wil ik graag mijn professoren, Jurriaan en Han, bedanken door mij de mogelijkheid te hebben geven een promotieonderzoek op dit interessante onderwerp te doen. Samen zijn jullie een sterk team begeleiders en vullen elkaars kwaliteiten goed aan. Ik heb altijd genoten van onze twee-wekelijkse besprekingen, waarin we efficiënt door de plannen en resultaten heen gingen. Het feit dat ik gedeeld werd tussen twee vakgroepen heb ik altijd als voordeel gezien, zowel wetenschappelijk (bredere expertise) als sociaal (twee keer zoveel taart en vakgroepuitjes). Jurriaan, vooral jouw directe en kritische manier van denken en begeleiden heeft me vaak geholpen om zo efficiënt mogelijk aan de slag te gaan, zonder de belangrijke aspecten uit het oog te verliezen. Han, je kan soms met slechts één zin of vraag een probleem in kaart brengen of zelfs een complete oplossing geven ("Waarom probeer je de methode van dit patent uit 1974 niet eens?'). Hierdoor is het schrijven van artikelen en het proefschrift ook erg vlot verlopen.

Naast de begeleiding van de professoren, heeft Roald mij ook erg veel geholpen. Roald, vooral in de beginfase van mijn onderzoek heb je mij veel geleerd op het gebied van werken in de cleanroom, waardoor we binnen notime op grote schaal pilaren fabriceerden. Naarmate het cleanroom-werk steeds beter ging, heb je alsnog je bijdrage geleverd in de talloze discussies van de nieuwste resultaten en het corrigeren van mijn artikelen. Mede door jou heeft het een aantal mooie publicaties opgeleverd. Vooral de manier waarop we het 
review-artikel uit het niks hebben opgezet, laat zien dat de samenwerking perfect verliep, met een artikel waar we beide erg trots op zijn (Hoofdstuk 2). Behalve op wetenschappelijk gebied, hebben we ook erg veel lol gehad met squash, bier en voetbal. Op één of andere manier raken we daar nooit over uitgesproken, en dit zal waarschijnlijk ook nooit gebeuren.

Tijdens mijn promotie heb ik ook twee masterstudenten begeleid, Nienke en Alexander, die ik wil bedanken voor hun inzet. Nienke, jij was op zoek naar een uitdagend project op het gebied van duurzame energie. Volgens mij is dat goed gelukt en, samen met Janneke, was de begeleiding ook erg makkelijk aangezien je heel gemotiveerd en enthousiast was. Dit is uiteindelijk ook terug te zien in je mooie verslag. Alexander, we had a lot of fun in the cleanroom trying to replicate a difficult pillar process. Your eagerness and persistence on the project made the guidance easy, with some nice results in only a few months' time. After your master project, I was happy to hear that you started a PhD position at Guido's group, and our collaboration continued and you assisted me in the electrical measurements that led to the first paper (Chapter 3). As you gained experience in your own project, we started a new experiment, which led to a nice paper about the combination of our separate projects, as seen in Chapter 6.

Ook wil ik graag mijn paranimfs bedanken, Jasper en Wouter. Jasper, hoewel onze projecten verre van overeenkomstig waren, hebben we altijd goed kunnen discussiëren over onze bevindingen, en hielden elkaar scherp. Op sociaal gebied hebben we een stuk meer samengewerkt, wat zich tot uiting bracht als geniale avonden met slechte films en presentaties over frituur. Ik ben blij dat we bijvoorbeeld de aziatische culltuur kennis hebben laten maken met New Kids en het ontstaan van de kroket. Ook onze coop-wandelingen waren zeer gezellig, en konden we beide onze verbazing over van alles en nog wat kwijt. Wouter, jij kwam ongeveer 1,5 jaar voor het einde van mijn PhD bij de MnF vakgroep, en we zijn eigenlijk direct aan de slag gegaan om voortgang te boeken. Je hebt veel geholpen met het meedenken van de vervolgstappen van mijn project, en hieruit is het werk in Hoofdstuk 4 uitgekomen. Met je eigen project kwam je toen 
ook al een stuk verder, en in Hoofdstuk 5 hebben we dit tot een mooi resultaat gebracht. Op persoonlijk gebied was ik ook erg blij dat je bij de vakgroep kwam, aangezien onze passie voor bier en squash goed overeen kwam, met als hoogtepunt om 7:00 squashen met een kater op Ameland tijdens het vakgroepsuitje. Klein minpunt is dat ik nog steeds moet vrezen voor mijn leven als we samen op de squashbaan staan.

Verder heb ik veel samengewerkt met de mensen betrokken bij het TBSC cluster op de UT; Janneke, onze projecten waren in het begin niet heel sterk verbonden, maar met Nienke's masteropdracht erbij hebben we toch een aantal leuke experimenten gecombineerd. Het blijft altijd typisch dat het laatste experiment voordat we er mee zouden stoppen, de meest interessante data gaf. Hopelijk gaat het nog ergens toe leiden, maar dat komt vast goed met jouw optimisme, in combinatie met je realiteit (volgens mij doet er niemand zoveel controle experimenten als jij ()). Sun-Young, we started together on the same project and tested many different options. Unfortunately, we were not that lucky to get everything to work as we wanted. You did help a lot with the measurements at PCS, for which I am thankful. Ook wil ik graag de andere leden van de TBSC groep bedanken voor alle discussies; Jennifer, Guido, Annemarie, David, Qing, Saskia, Samuel, Peter, Sebastiaan.

Aangezien ik deel uitmaakte van twee verschillende vakgroepen (MnF en MCS) en vaak rondliep bij PCS, zijn er een hoop mensen die mij ondersteund hebben met veel verschillende dingen; Nicole, Izabel, Richard, Marcel, Robert, Stefan, Brigitte, Jacqueline, Bianca en Regine. Nicole en Izabel, jullie wil ik bedanken voor het regelen van alle organisatorische zaken rondom FOM, en het beantwoorden van talloze vragen. Marcel, jij snapte mijn ergernis dat er geen cola-automaat op de $4^{\text {de }}$ verdieping van Carré staat, en hebt dit goed opgelost door een koelkast te vullen op jouw kantoor, waar ik veelvuldig gebruik van heb gemaakt.

Ook wil ik graag de SaNS-stafleden bedanken voor het regelen dat de vakgroepen een fijne plek waren om te werken; Wim, Jeroen, Pascal, Nathalie 
en Tibor. Pascal, jou wil ik in het bijzonder bedanken, omdat jij met de begeleiding van mijn bachelor- en masterproject een grote bijdrage hebt geleverd aan mijn interesse voor het wetenschappelijk onderzoek.

Veel van mijn experimenten heb ik in de cleanroom van het Nanolab uitgevoerd, en het ondersteunende personeel heeft mij daarbij veel geholpen; Peter, Hans, Ite-Jan, Samantha, Marion, Huib, Mark, Gerard, Eddy, Ton, Robert, Rene, Meint, Christiaan en Sharron wil ik daarvoor bedanken. Hans, Peter en Rene, jullie wil ik speciaal bedanken voor alle vragen die ik tijdens mijn experimenten had, en vooral voor het oplossen van talloze problemen met de apparaten, maskers e.d. In het bijzonder wil ik Kees en Stefan bedanken, die mij meerdere malen met problemen/processen hebben geholpen als medegebruikers in de cleanroom.

As a PhD takes 4 years, there are many people I have encountered and who left the group before me and I would like to thank a few in particular: Pieter, Carmen, Sven, Rik, Wies, Raluca, Rajesh, Kim, Carlo and Jordi. I would also like to thank all current members of MnF and MCS, but I will not mention every single one of them, as the list would become extremely long $(;)$.

Tot slot wil ik graag familie en vrienden bedanken, die mij op allerlei verschillende manieren geholpen hebben. Pap en mam, jullie wil ik graag bedanken voor alles wat jullie voor me betekend hebben. Dit valt niet uit te leggen in een paar zinnen, maar jullie hebben me altijd ondersteund in alle keuzes die ik gemaakt heb. Als laatste wil ik graag mijn vrouw, Alexandra bedanken. Alex, jij hebt er voor gezorgd dat ik in mijn privéleven nooit zorgen heb hoeven maken over wat dan ook. We zijn alweer negen jaar samen, en tegelijk met het begin van mijn PhD zijn we samen gaan wonen. Afgelopen jaar zijn we getrouwd, en ik kijk erg uit naar wat de toekomst ons samen nog allemaal te bieden heeft.

Rick, Enschede, November 2015 


\section{Curriculum Vitae}

Rick Elbersen was born January 6, 1987 in Deurne, the Netherlands. In 2005, he started his bachelor chemical engineering at the University of Twente. $\mathrm{He}$ received his bachelor degree in 2009, after which he continued with his master Molecules \& Materials. In 2011, he graduated for his master assignment under the supervision of Prof. dr. Pascal Jonkheijm and Prof. dr. ir. Jurriaan Huskens at the Molecular NanoFabrication group. The title of the project was 'Charactization of adamantane functionalized perylene bisimides for the detection of $\beta$-cyclodextrin modified molecules'.

Starting from July 2011, he has been working as a PhD candidate at the Molecular Nanofabrication group and the Mesoscale Chemical Systems group at the University of Twente, under the supervision of Prof. dr. Jurriaan Huskens and Prof. dr. Han Gardeniers. The aim of the project was the fabrication and improvement of silicon solar cells. The results of his PhD work are described in this thesis. 


\section{Publications}

1. R. Elbersen, R.M. Tiggelaar, A. Milbrat, J.G.E. Gardeniers, J. Huskens; "Controlled Doping Methods for Radial $p / n$ Junctions in Silicon Micropillars" Adv. Energy Mater., 2015, 5, 1401745

2. R. Elbersen, W.J.C. Vijselaar, R.M. Tiggelaar, J.G.E. Gardeniers, J. Huskens;

"Fabrication and Doping Methods for Silicon Nano- and Micropillar Arrays for Solar Light Harvesting: a Review"

Adv. Mater., 2015, doi: 10.1002/adma.201502632

3. R. Elbersen, W.J.C. Vijselaar, R.M. Tiggelaar, J.G.E. Gardeniers, J. Huskens;

"Effects of Pillar Height and Junction Depth on the Performance of Radially Doped Silicon Pillar Arrays for Solar Energy Applications" Adv. Energy Mater., 2015, accepted

4. A. Milbrat, R. Elbersen, R. Kas, R.M. Tiggelaar, J.G.E. Gardeniers, J. Huskens, G. Mul;

"Spatioselective Electrochemical and Photoelectrochemical Functionalization of Silicon Microwires with Axial p/n Junctions"

Adv. Mater., 2015, accepted

5. W.J.C. Vijselaar, R. Elbersen, R.M. Tiggelaar, J.G.E. Gardeniers, J. Huskens;

"Electrical Characterization of Silicon Micropillars with Radial $p / n$ Junctions Containing Passivation and Anti-Reflection Coatings" in preparation 